UCRL- -53334

DE83 001483

\title{
Fluorescence Line-Narrowing Studies of Rare Earths in Disordered Solids
}

\author{
Douglas W. Hall
}

Manuscript date: August 10, 1982

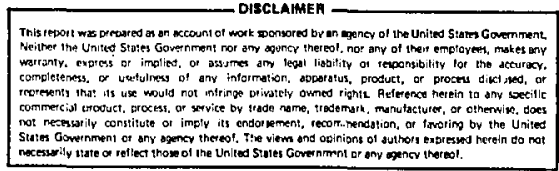

\section{NOTICE}

PORTIONS OF THIS REPORT ARE ILLEGIBLE it has been reproduced from the best availablo copy to permit the broadest possible avaik sbilly.

\section{LAWRENCE LIVERMORE LABORATORY} University of California • Livermore, California 94550 
By

DOUGLAS WARREN HALL

B.A. (Occidental College) 1975

M.S. (University of Calffornia, Davis) 1979

\section{DISSERTATION}

Submitted in partial satisfaction of the requirements for the degree of DOGTOR OF PHILOSOPHY

in

Engineerling-Applied Science

in the

GRADUATE DIVISION

of the

UNIVERSITY OF CALIFORNIA

DAVIS

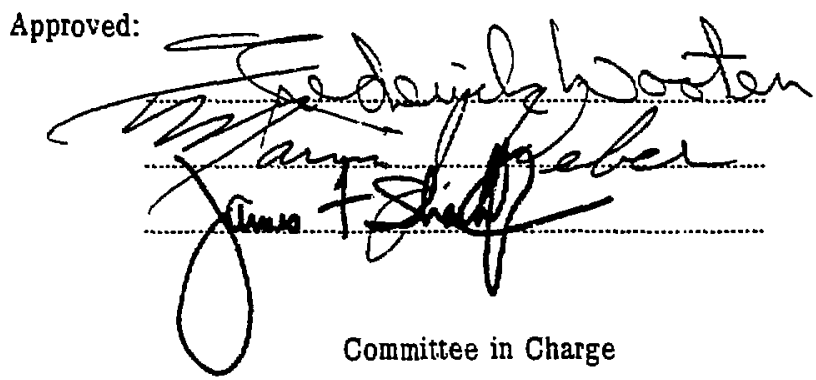

Deposited in the University Library 
Table of Contents

Page

Part I

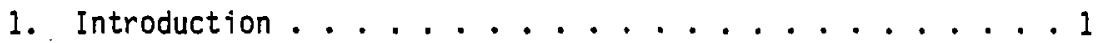

2. Survey of Optical Properties of Rare Earths in Solids. . . . 5

\section{Part II}

3. Vibronic Spectra of $\mathrm{Gd}^{3+}$ in Disordered Solids ..... . 26

4. Experimental Technique .............. 45

5. Glass Results and Discussion............. . 52

6. Crystal Results and Discussion ........... 83

\section{Part III}

7. Orientational and Spectral Inhomogeneity in Nd:Laser Glass . . . . . . . . . . . . 96

8. Experimental Details and Results ........... 105

9. Model Calculations ............... . 129

10. Comparison of Model Calculations with Saturation Experiments ............. 168 


\section{Appendices}

A. Kramers-Kronig Transform . . . . . . . . . . . . 198

B. Amplifier Calculation Computer Programs . . . . . . 216 
$-1-$

PART I 


\section{Chapter 1: INTRODUCTION}

This dissertation is made up of two experimental studies dealing with apparently diverse topics within the subject of rare earths (RE) in solids. The first study, described in Part II, concerns the vibrations of a disordered host material about an optically active rare-earth ion as manifested by vibrationally-assisted-electronic, or "vibronic" transitions. These vibrations influence an ion's optical properties in several ways: they affect excited state lifetimes, quantum efficiencies, and the homogeneous linewidth of transitions. Conversely, an ion's vibronic fluorescence transitions can reveal a great deal about the host's vibrations. Vibrations in disordered solids are not well understood, mostly because these materials lack the periodicity required for the mathematical simplification and analytical models of perfect crystals. The vibronic spectrum of the ion $\mathrm{Gd}^{3+}$ is shown to be a valuable localized probe of these vibrations that complements the more traditional, bulk material vibrational spectroscopies; e.g. Raman scattering and infrared reflection.

A major question addressed in Part II concerns the nature of the vibrations that couple to the ion's electronic transitions: are they characteristic of the bulk material or do they result from a perturbation of the host caused by the massive, highly charged rare earth? To answer this question, the vibronic spectrum of $\mathrm{Gd}^{3+}$ was recorded in a series of metaphosphate glasses in which the mass, charge, and size of the RE dopant were either similar or dissimilar to those of the glass modifier cation for which they substitate. Infrared and Raman spectra, which are 
characteristic of the undoped media, were also measured to provide information about bulk material vibrations.

Another question pertains to the site-to-site variation that rare-earth ions experience in glass: are vibronic spectra sensitive to changes in the local ionic environment? A careful study of vibronics of $\mathrm{Gd}^{3+}$ in $\mathrm{BeF}_{2}$, a simple glass former, was undertaken to answer this question.

To demonstrate the utility of using vibronics as a probe of vibrations in crystals, we examined $\mathrm{Gd}^{3+}$ doped $\mathrm{CaF}_{2}$. Different charge compensation mechanisms for the trivalent RE dopant make this crystal a disordered solid. Vibronic spectra of ions with the various types of compensation may be obtained.

Part III of the dissertation describes an investigation of the influence of site anisotropy on the purely electronic, laser transition of $\mathrm{Nd}^{3+}$ in glass. The low symmetry of ion environments in glass can cause an ion to interact more readily with certain polarizations of optical radiation; this effect may be described by associating an angle-dependent stimulated emission cross section with each RE. Aithough RE environments in glass may be thought of as an isotropically oriented collection of these anisotropic sites, one consequence of an angle-dependent cross section is a "polarization hole burning" that becomes evident in glass laser amplifiers operated in the large-signal regime. This hole burning is analogous to the more well-known spectral hole burning that results from site-to-site variation in transition 
frequency. Both orientational and spectral inhomogeneities must be included in analyses used to predict the performance of glass amplifiers operated 'in this regime.

These two studies are bound together by the common experimental technique of laser-induced fluorescence line narrowing (FLN). By exciting fluorescence with monochromatic light of well-characterized polarization, one may select and observe the response of a single subset of the optically active ions and obtain information that is usually masked by the inhomogeneous nature of disordered solids.

The background necessary for an understanding of the optical properties of REs in solids, which is common to both studies, is presented in survey form in Chapter 2. Some general features of the FLN tèchnique are also described. 
Chapter 2: SURVEY OF OPTICAL PROPERTIES OF RARE EARTHS IN SOLIOS

The optical spectra of rare-earth ions ${ }^{1}$ in transparent solids are characterized by narrow absorption and emission lines which are reminiscent of spectra from free atomic or ionic systems. In fact, the energy level separations of a RE ion in a solid host are usually found to differ from those of the ion in the gas phase ${ }^{2}$ by less than $200 \mathrm{~cm}^{-1}$, while typical level spacings are on the order of $10^{3}$ to $10^{4} \mathrm{~cm}^{-1}$. For example; the three lowest energy spectral lines of $\mathrm{Gd}^{3+}$ are listed in Table I for the free ion and for the ion in two crystals and a glass. Free-ion data was obtained from arc discharge emission, ${ }^{3}$ while crystal ${ }^{4}$ and glass data was obtained from transmission measurements. Absorption due to $\mathrm{Gd}^{3+}$ ions in the glass host is shown in the transmission spectrum of Figure 1 with lines denoted by the free-ion quantum label of the final state (transitions originate from the ion's ground state). The steady decrease in transmission with decreasing wavelength from $300-200$ $\mathrm{nm}$ arises from reflection losses related to electronic transitions across the intrinsic band gap of the host.

The effect of host on RE energy leveis is small because these levels originate from intraconfigurational changes of orbitals of the partially filled $4 f$ shell. A majority of the wave functions of these orbitals lies inside the closed $5 s$ and $5 p$ shells and they are well shielded from the environment external to the ion. ${ }^{5}$ Thus, a theoretical understanding of RE energy states and transitions in solids may be obtained by treating the effect of environment as a perturbation acting on free-ion eigenstates. 
Table I. Energy levels of $\mathrm{Gd}^{3+}$ (in $\mathrm{cm}^{-1}$ ) for different environments; values for solid hosts represent the center of gravity of the observed line.

\begin{tabular}{ccccc}
\hline \hline Excited quantum state & Free ion & $\mathrm{LaF}_{3}$ & $\mathrm{CaF}_{2}$ & $\mathrm{Gd}\left(\mathrm{PO}_{3}\right)_{3} \mathrm{glass}$ \\
${ }^{6} \mathrm{P}_{7 / 2}$ & 32000 & 32195 & 32133 & 32051 \\
${ }^{6} \mathrm{P}_{5 / 2}$ & 32616 & 32789 & 32701 & 32626 \\
${ }_{6}{ }_{\mathrm{P}}$ & 33209 & 33363 & 33311 & 33222
\end{tabular}

$\begin{array}{ll}\text { Ref. } 3 & \text { Ref. } 4 \\ & T=4 K\end{array} \quad T=293 K \quad T=293 \mathrm{~K}$




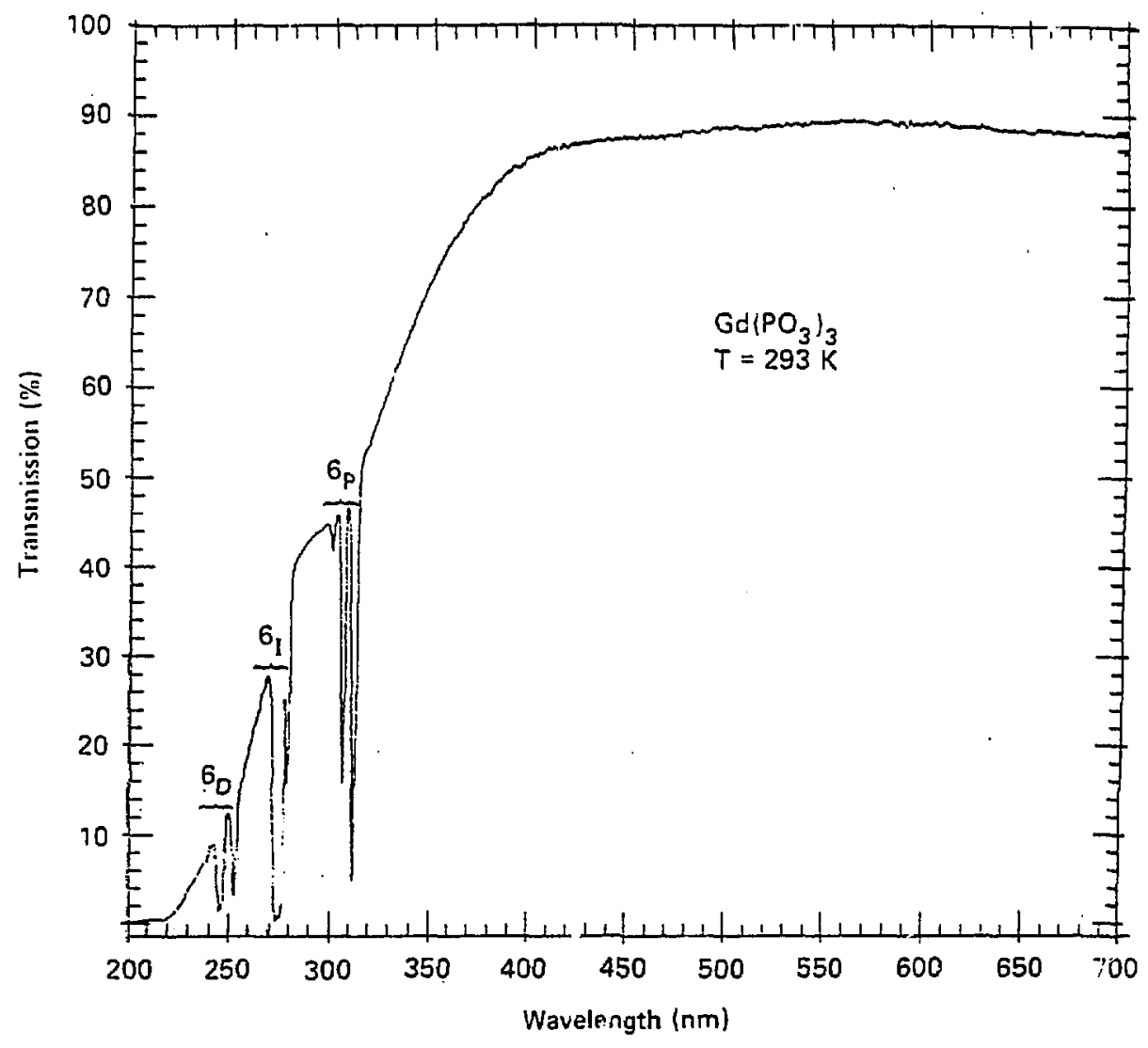

Figure 1: Optical transmission of Gadolinium metaphosphate glass. Sample thickness $=0.71 \mathrm{~cm}$. 


\section{A. Free RE Ion States ${ }^{5-8}$}

The Hamiltonian appropriate for calculating the eigenstates and eigenenergies of a free many-electron atom or ion is

$$
\begin{aligned}
H & =\sum_{i=1}^{N}-\frac{\hbar^{2}}{2 m} \nabla_{i}^{2}-\sum_{i=1}^{N} \frac{Z e^{2}}{r_{i}}+\sum_{i<j} \frac{e^{2}}{r_{i j}}+\sum_{i=1}^{N} \zeta\left(r_{i}\right) T_{i} \cdot \vec{s}_{i} \\
& =H_{k}+H_{n}+H_{e e}+H_{s o},
\end{aligned}
$$

Where $N$ is the total number of electrons, $H_{k}$ represents their kinetic energy, $H_{n}$ is the potential energy of their attraction to the nucleus, $H_{e e}$ is the energy of electrostatic repulsion between slectrons, and $H_{\text {so }}$ is the energy of interaction of the magnetic moment associated with each electron's spin and the magnetic field created by its orbital motion (i.e. the spin-orbit interaction). Terms representing other interactions (e.g. spin-spin, spin-other orbital) are small and are ignored.

Solution of Eq. (1) proceeds by making the central-field approximation: each electron is assumed to move independently in the field of the nuc?eus and a central field arising from the spherically averaged charge of the electrons. Equation (1) may be written as

$$
\begin{aligned}
H & =H_{k}+\sum_{i=1}^{N} U\left(r_{i}\right)-\left[\sum_{i=1}^{N} \frac{Z e^{2}}{r_{i}}+U\left(r_{i}\right)\right]+H_{e e}+H_{s a} \\
& =H_{c f}+H_{p}+H_{e e}+H_{s o},
\end{aligned}
$$

where $U\left(r_{j}\right)$ is the central-field potential energy. 
Equation (2) is solved approximately by finding the eigenstates of the central-field Hamiltonian $H_{c f}$, and treating $H_{p}, H_{e e}$ and $H_{s o}$ as perturbations. Essentially, one forms the matrix of the perturbation operators in the basis of central-field eigenstates and makes the linear combinations required to diagonalize the matrix. ${ }^{9}$ Eigenstates of $\mathrm{H}_{c f}$ may be expressed in terms of the antisymmeterized products of one-electron wavefunctions, which are usually obtained by the Hartee-Fock method. The one-electron wavefunctions are similar to hydrogenic wavefunctions in that they are a product of a radial function and a spherical harmonic. The eigenvalues of $H_{c f}$ are highly degenerate, with each one corresponding to a specific configuration of one-electron states. A configuration is labelled by its orbitals' hydrogen-like radial and angular momentum quantum numbers and the number of electrons in each orbital. For levels of the trivalent REs within the optical energy range, calculation of perturbation matrix elements are usually restricted to states of a single configuration. The relevant configuration is that of the xenon atom with additional filled $4 f$ orbitals, denoted by $4 f^{n}$, where $n$ varies from one to 14 across the Lanthanide series (e.g. for $\mathrm{Nd}^{3+}, \mathrm{n}=3$; for $\mathrm{Gd}^{3+}, \mathrm{n}=7$ ).

The radial functions for the many-electron states of a given configuration are identical in the central-field approximation so that matrix elements of operators that depend only on the coordinates $r_{i}$ (i.e. operators that are spherically symmetric) contribute energy shifts that are the same for all states. Since one measures only the difference in energy between eigenstates in optical experiments, we may ignore the 
effect of this type of operator; e.g. $\mathrm{H}_{p}$. Matrix elements of $\mathrm{H}_{e e}$ and $H_{\text {so }}$, however, will be different for different states of the same configuration and will lift the degeneracy of the central-field wavefunctions.

So far, evaluation of matrix elements has implied integration over the coordinates of all $\mathrm{N}$ electrons of the ion. It may be shown ${ }^{10}$ that matrix elements of operators using many-electron wavefunctions of all electrons and those calculated using wavefunctions made up only of orbitals of shells that are not closed differ only by a constant factor. This is because the closed-shell portion of each $\mathrm{N}$-electron wavefunction of the configuration is identical. Thus, in considering energy leve 1 differences of the $4 f^{n}$ configuration, we need only be concerned with Wavefunctions of the $4 f$ electrons.

The many-electron wavefunctions discussed so far are simultaneous eigenfunctions of the following set of commuting operators: $\mathrm{H}_{\mathrm{cf}}$, $l_{i}^{2}, s_{i}^{2}, 1_{i z}$, and $s_{i z}$. Linear combinations of these functions can be formed that are eigenfunctions (or approximate eigenfunctions) of other sets of commuting operators. Calculation of matrices of operators in judiciously chosen basis sets great ly reduce the number of non-zero matrix elements. One such basis set is made up of eigenfunctions of the operators $H_{c f}+H_{e e}, L^{2}, S^{2}, L_{z}$ and $S_{z}$, where $\vec{L}$ and $\vec{S}$ are the total orbital and spin angular momenta, respectively. We ignore the small amount of mixing of other configurations required to obtain exact eigenfunctions of $\mathrm{H}_{c f}+\mathrm{H}_{e e}$. Another useful basis set is arrived at by assuming that $\mathrm{H}_{c f} \gg H_{e e} \gg H_{\text {so }}$ and that $H_{\text {so }}$ may be 
approximated by a term proportional to $\vec{L} \cdot \vec{S}$. Then eigenfunctions of the operators $\mathrm{H}_{\mathrm{cf}}+\mathrm{H}_{\mathrm{ee}}+\mathrm{H}_{\mathrm{so}}, \mathrm{L}^{2}, \mathrm{~S}^{2}, \mathrm{~J}^{2}$ and $\mathrm{J}_{\mathrm{z}}$ are formed where $\vec{j}$ is the total angular momentum. These are the Russe1l-saunders (RS) eigenfunctions of atomic theory. 10

Eigenvalues and eigenstates in the RS approximation are labelled by $2 S+l_{\mathrm{L}}$ where $S$ is the total spin quantum number, $L$ is the letter that designates total orbital angular momentum quantum number $(S, P, D, \ldots=$ $0,1,2 \ldots$.$) , and \mathrm{J}$ is the total angular momemtum quantum number. States with the same ${ }^{2 S+l_{L}}$, label but with different values of $J_{2}$ quantum number are $(2 J+1)$-fold degenerate.

For REs, it turns out that $\mathrm{H}_{c f} \gg \mathrm{H}_{e e} \gtrsim \mathrm{H}_{\text {so }}$ so that the Russe 17-Saunders approximation is not valid and the entire perturbation matrix, $H_{i c}=H_{e e}+H_{\text {so }}$, must be calculated and diagonalized. This is the intermediate coupling (IC) case and the oniy angular momentum operators that commute with $\mathrm{H}_{c f}+\mathrm{H}_{i c}$ and provide "good" quantum numbers are $J^{2}$ and $J_{z}$ (with states degenerate in $J_{z}$ ). It is common practice to use RS eigenfunctions as a basis set for the diagonalization. Thus, IC eigenfunctions are expressed as a linear combination of RS states and are labelled by the quant $r$ numbers of the dominant RS state (this is the origin of the labels in Table I):

$$
\left[f^{n}\{\gamma S L\} J\right\rangle=\sum_{\gamma S L} c(\gamma S L)\left[f^{n} \gamma S L J\right\rangle
$$

$\gamma$ represents other quantum numbers of the RS approximation. Eigenvalues labelled with the same $\gamma, S$ and $L$, but with different J-values, are usually found to be separated by an energy on the order of $10^{3}$ to $10^{4} \mathrm{~cm}^{-1}$. 
We have out?ined a method for obtaining and denoting a useful set of free-ion eigenstates for trivalent RE ions, each of which may be separated into a product of functions of the radial coordinates of the electrons and functions of their angular coordinates. The angular coordinate function is a linear combination of products of the well-defined spherical harmonic functions for the individual electrons. Elaborate methods, such as tensorial operator techniques, ${ }^{11}$ have been developed for evaluating exactly the contribution of angular functions to matrix elements of perturbations acting on the free ion. This allows predictions of the degeneracy of energy states for certain problems as well as selection rules for transitions caused by various types of radiation. The radial functions are usually less well-specified and radial integrals in matrix elements are often parameterized with values determined from experiment. An example of radial integral parameters are the Racah parameters ${ }^{5}$ used in evaluating matrix elements of electrostatic interactions.

B. RES in Solids

The crystal field

When a RE is incorporated into a solid, it experiences an electrostatic field created by the charge distribution of the ions and electrons that make up the host. The effect of the field on the $4 f$ electrons of the RE is described by a potential energy function, $V$, usually represented in terms of what is essentially an expansion in spherical harmonics:

$$
V=\sum_{i=1}^{n} \sum_{k q} B_{k q}\left(C_{q}^{k}\right)_{i}
$$


where $C_{q}^{k}$ is a component of the tensor operator $C^{k}\left(C_{q}^{k}\right.$ is simply related to the spherical harmonic $\left.Y_{k q}\right) ; B_{k q}$ is a function of the radial coordinate of the f electrons and is usually treated as a parameter. 2,5 For REs, $\mathrm{H}_{\text {ee }} \geq \mathrm{H}_{\text {so }} \gg \mathrm{V}$ so that the effect of the surrounding host may be treated as a small perturbation acting on free-ion states, usually with first-order perturbation theory. The fact that we are dealing with only fi electrons $\left(1_{i}=3\right)$ limits contributions to matrix elements of $v$ to terms with $k \leq 6$. This is because the matrix elements eventually involve integrands of the form $\left(Y_{1 m}\right)^{*} Y_{l m}, Y_{k q}$ and it can be shown ${ }^{12}$ that the $\theta$ portion of the integrals are zero unless $k \leq 2 \cdot 1$. Other rules, such as $m+m^{\prime}+q=0$, pertain to integrals of this type. The symmetry of a RE site may further reduce the number of terms in the expansion of $V$ as dictated by group theory. For example, a site of cubic symmetry, $O_{h}$, is described by a crystal potential that contains only a few of the $k=4$ and $k=6$ terms (the $k=$ 0 term is spherically symmetric, introduces a constant energy shift for all states of the $4 f^{n}$ configuration, and may be ignored). Sites in glass are expected to be of $C_{1}$ symmetry and require all terms with $k \leq$ 6.13 The electrostatic field generated by host ions is, for historical reasons, called the crystal field, even though the term is also applied to the field at RE sites in glass.

It should be noted that, because of the ordering of perturbations $\left(H_{e e} \geq H_{s o} \gg V\right)$, REs are treated in what is called the weak-field case of crystal field theory. Transition ions of the iron group (with an. unfilled $3 d$ shell) fall into the medium-field case $\left(\mathrm{H}_{\mathrm{ee}}>\mathrm{V}>\mathrm{H}_{\text {so }}\right)$, 
while palladium and platinum group ions (unfilled $4 d$ and $5 d$ shells, respectively) fall into the strong-field case $\left(V>\mathrm{H}_{e e}>\mathrm{H}_{\text {so }}\right)$. Theoretical techniques for the different cases vary considerably. ${ }^{7}$ Energy leve $1 \mathrm{~s}$

The crystal field splits the $2 J+1$ degenerate free-ion levels into a number sut-levels, with the number dictated by the symmetry of the inhomogeneous field. This splitting is analogous to that of vapor phase atoms or ions in an externally applied electric field, i.e. the Stark effect, and the resulting states in a solid are called Stark states. The Stark splitting of a J-level of a RE is on the order of 10 to $100 \mathrm{~cm}^{-1}$. In low-symmetry sites (e.g. $C_{1}, C_{2 V}$ ), the entire $2 J+1$ degeneracy of a. free-ion eigenenergy is removed for ions with an even number of electrons, while ions with an odd number of electrons are spiit into $J+\frac{1}{2}$ two-fold degenerate states. The residual degeneracy of odd-electron ions (e.g. $\mathrm{Nd}^{3+}$ and $\mathrm{Gd}^{3+}$ ) in an electric field is the result of the time-reversal symmetry of the Hamiltonian and is called the Kramers' degeneracy. ${ }^{7}$. Transitions between Stark states

The free-ion states described in Section A were restricted to product wavefunctions from the $4 f^{n}$ configuration. This results in total wavefunctions which all have the same farity. Transitions between these states by an electric-dipole process should be forbidden and the lowest order process possible should be by magnetic-dipole interaction. Most transitions of RE ions in solids, however, occur by the electricdipole process. ${ }^{14}$ Hence, there must be some mixing of apposite parity configurations (e.g. $4 f^{n-1} 5 d$ ) into the wavefunctions. This mixing can 
occur if the RE ion experiences a crystal field that lacks inversion symmetry, producing odd-order terms in $V$. The amount of admixing is small, as demonstrated by typical radiative lifetimes on the order of 100 to $1000 \mu s$ (a fully allowed electric-dipole transition has a radiative lifetime on the order of $1 \mathrm{~ns})$.

For a single isolated ion, all transitions between states must be accompanied by the emission or absorption of a quantum of electromagnetic energy, a photon. For ions in a host, nonradiative transitions ure possible in which vibrations in the lattice (phonons) supply or absorb the energy of the electronic change of state. One manifestation of the weak ion-phonon interaction of REs is vibronic sidebands which will be treated in more detai] in Chapter 3. An important consequence of the ion-phonon interaction is that transitions occur very rapidly between electronic states that are separated by less than the solid's maximum phonon energy (i.e. transitions occur on a time scale of a vibration period, $\ll 1$ ps); hence, transitions between these states are completely dominated by this nonradiative process. States separated by greater energies ( $\gg 100 \mathrm{~cm}^{-1}$ ) make transitions either radiatively or nonradiatively by less probable multiphonon processes.

One result of rapid nonradiative transitions is that lines that appear in absorption spectra of REs need not appear in fluorescence spectra. If an ion is excited to a high-energy state that has lower energy states close to it, the ion will relax nonradiatively, cascading downward in energy until it reaches a "metastable" state with an energy gap that is large enough to make radiative transitions probable. 
Fluorescence is observed from these states. Another result is that an ensemble of ions will have their energy levels populated according to Boltzmann's statistics, with a temperature dictated by that of the solid. An ensemble of ions elsvated to an excited state with a lifetime which is long on the vibrational time scale will also make transitions so that the close-lying states are "locally thermalized", i.e. populated according to the Boltzmann probability distribution. Fluorescent transitions from each of the close-lying states to lower-energy states is then possible.

Another nonradiative iransiti I mechanism that occurs in systems where the density of REs is sufficient to allow interaction between them is the process of cross-relaxation. 15 An ion in an excited state may relax to a lower state with energy conserved by an upward transition in a neighboring ion. The probability of this type of event is greatest when the energies of the transitions of the respective ions are closely matched; small energy differences may be mediated by phonons. The states of the two ions that are involved may be the same or different. A consequence of cross-relaxation when the states are the same is energy migration which results in spatial and spectral equilibration of excitation; spectral equilibration will be discussed further in Section 0 . A consequence of the ion-ion interaction when states are different is the quenching of what would be radiative transitions in less concentrated systems.

The fact that RE ions in transparent solids absorb broadband light by transitions to many excited states and then rapidly relax to one (or 
several) metastable state makes them ideal candidates for optically pumped laser media. The spontaneous radiative process of fluorescence implies, the possibitity of stimulated emission necessary for laser action. The probability of stimulated emission is characterized by a cross section, $\sigma$, which, for a transition of Lorentzian spectral line shape of width $\Delta \nu$, is given by

$$
\sigma=\frac{\lambda_{0}^{2}}{(2 \pi n)^{2} \Delta \nu} \frac{1}{T}
$$

where $\tau$ is the radiative lifetime of the transition, $\lambda_{0}$ is the vacuum wavelength of emission, and $n$ is the index of refraction of the medium. ${ }^{16}$ This expression must be slightiy modified for the different lineshapes observed in solids. 17

If a population inversion density, $N$, is obtained by optically. pumping the medium, then an incident beam of vacuum wavelength $\lambda_{0}$ and intensity $I$ will be amplified with a gain in intensity with distance given by

$$
\frac{d I}{d r}=(\sigma N) I
$$

The quantity in parenthesis is the gain coefficient, usually denoted by $\dot{g}$. C. Spectral Line Broadening

Features in absorption and emission spectra due to purely electronic transitions of REs in solids possess finite widths which are attributable to several processes. Each process may be categorized as either a 
homogeneous or inhomogeneous mechanism. A homogeneous broadening mechanism contributes to the linewidths of transitions observed from a single ion. Inhomogeneous broadening of lines in solids results from the superposition of the homogeneous ly broadened lines from ions that have different local environments and thus different spectral properties. Homogeneous broadening

Homogeneous broadening is a result of the $f$ inite lifetime of the states involved in an electronic transition. ${ }^{14}$ The energy of a state becomes less precisely defined is the state's lifetime decreases, in accordance with the Heisenberg uncertainty principle; the homogeneous width of a spectral line reflects the uncertainty in energy of both the initial and final states.

Lifetime-reducing processes include the radiative and nonradiative ones of the previous section, with nonradiative processes being dominant for most states. For example, a typical RE radiative lifetime of $1 \mathrm{~ms}$ corresponds to a linewidth of $10^{-7} \mathrm{~cm}^{-1}$ while the homogeneows linewidths of transitions at room temperature are on the order of one to $100 \mathrm{~cm}^{-1}$. An additional process that does not involve transitions but still limits the lifetime of a state is associated with "collisions" of the RE with surrounding host ions; these can result in a phase disruption of the quantum state. 18

Phonon related processes are very temperature dependent and one finds that the homogeneous linewidths of transitions involving metastable states are aiso extremely temperature dependent. ${ }^{7}$ Higher-energy close-lying states (e.g. all but the lowest state of a Stark split level) are not as affected since their lifetimes are limited at low-temperatures by transitions via spontaneous phonon emission. 
Inhomogeneous broaden ing $^{13}$

Ions in solids experience different local environments; in crystals, this is attributable to various mechanisms of charge compensation, to growth strains, dislocations, etc. while in glasses it is due to the disordered nature of the media. Mathematically, the site-to-site variation is represented by a distribution of values of the coefficients $B_{k q}$ in Eq. (4) which implies that the energy levels of ions in d: ferent sites will vary. For crystals, this often results in spectra with a greater number of lines than predicted by the removal of degeneracy of a J-level by the crystal field. For glasses, where the inhomogeneous variation of energy levels about a mean value is often on the order of the Stark splitting, this results in broad spectral lines that are the superposition of many unresolved components.

Homogeneous linewidths and both radiative and nonradiative transition probabilities are also affected by the site-to-site variation in environment. In glasses, for example, the variation of total transition probability of a state is apparent in observed fluorescence decay times that are non-exponential, even though the decay of a class of ions with an identical local environment is exponential. Decay rates are measured in "e-folding times" (the time interval for the fluorescence intensity to reach $e^{-1}$ of its previous value); these steadily increase as the relative proportion of rapidly relaxing ions decreases with respect to the longer-lived ions.

The homogeneous and inhomogeneous contributions to spectral linewidths cannot be distinguished in absorption and broadband-excited 
fluorescence measurements. For example, one cannot tell if a broad band in an absorption spectrum is due to a single transition with a large homogeneous linewidth or to a superposition of narrow lines with frequencies distributed so as to obtain the observed line shape.

D. Fluorescence Line Narrowing

When fluorescence is excited by a source of narrow spectra? width (in practice, a laser), a single subset of ions with energy levels separated by a frequency equal to that of the source is selected from the inhomogeneously troadened absorption profile and the response of this subset may be observed. Spectral features are of ter very much narrower than when broadband excitation is employed and the technique is called laser-induced fluorescence line narrowing ${ }^{13}$ (FLN). The technique has been used to explore many phenomena usually masked by inhomogeneous broadening such as site-to-site variations in local fields, electron-phonon coupling, and ion-ion interaction.

FLiN experiments are either resonant or nonresonant. In a resonant experiment, fluorescence is observed at the same frequency as the exciting laser; in a nonresonant experiment, frequencies due to fluorescence from other transitions of the selected ions are observed.

Several mechanisms can defeat the line narrowing and selective character of FLN experiments. If excitation occurs in a region of an absorption band where transitions have large homogeneous linewidths, many ions with a variety of line-center frequencies will be excited. Lowering sample temperatures and exciting in the low-frequency wings of bands where absorption is via the lowest-energy Stark state of a level allows maximal line narrowing. 
Ion-ion interaction can also frustrate line narrowing. An ion of the. or iginally excited subset may transfer it energy to a neighboring ion with significantly different spectral properties, Time-resolved FLN techn ques have bee:: employed to examine cross-relaxation and associated phen.mena. ${ }^{13}$ A spectrum taken imnediately after excitation often has only a sharp fluorescence peak due to the subset of ions resonant with the laser; subsequ ant spectra may lose the paak and evolve into spectra similar to one that was broadband excited as the remaining excited-state energy is shared among all the REs of the solid. Maximoi line narrowing in spectra máy be achieved by limiting the period of collection of fluorescence signal or by using samples with RE concentrations sufficientiy low so that cross-relaxation is improbable.

Accidental coincidences of energy levels is a third mechanism that may defeat line narrowing. An example of an accidental coincidence is illustrated by Figure 2, which shows the energy level diayrams of shree $\mathrm{Gd}^{3+}$ ions in different sites. A laser tuned to excited site B via : transition to the lowest-energy Stark-spiit-state of the ${ }^{\sigma_{p}}{ }_{7 / 2}$ lever, will also excite Site A via its second Stark state; Site $C$ wi' l not be excited. Thus, sites with two different local environments and quite different energy levels may be simultaneously excited, with both contributing to fluorescence spectra. The effect of this type of accidental coincidence may be minimized by exciting only into the low-energy wing of an absorption band. Accidental coincidences in which sites with differeni local environments have coincident. energy levels are possible and cannot be circumvented. This lowers the site-selective capability of FLN experiments and will be discussed further in Chapter 5 . 

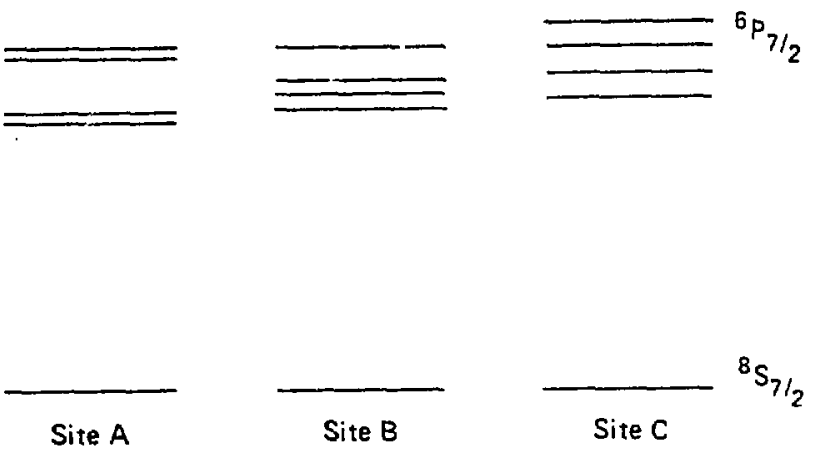

Figure 2: Schematic diagram of energy levels of $\mathrm{Gd}^{3+}$ ions in three different sites of a solid host. 


\section{References}

1. In this dissertation, the term "rare earth" is restricted to mean only elements of the Lanthanide series $(\mathrm{Ce} \rightarrow \mathrm{Lu})$. The Actinides $(T h \rightarrow L W)$, in which the $5 f$ orbital is filled across the series, are often referred to as rare-earth elements, especially in older literature.

2. S. Hlifner, Optical Spectra of Transparent Rare Earth Compounds, (Academic Press, New York, 1978).

3. J. F. Kielkoph and H. M. Crosswhite, J. Opt. Soc. Am. 60, 347 (1970).

4. H. H. Caspers, S. A. Miller, H. E. Rast, and J. L. Fry, Phys. Rev. $180,329(1969)$.

5. B. G. Wybourne, Spectroscopic Properties of Rare Earths, (John Wiley, New York, i965).

6. R. G. Pappalardo, in Luminescence of Inorganic Solids, ed. B. DiBartolo, (PTenum, New York, 1978), p. 178.

7. B. DiBartolo, Optical Interactions in Solids, (John Wiley, New York, 1968).

8. B. G. Wybourne, J. Opt. Soc. of Amer. 55, 928 (1965).

9. L. I. Schiff, Quantum Mechanics, 3rd Ed., (McGraw-Hi11, New York, 1968).

10. E. U. Condon and G. H. Shortley, The Theory of Atomic Spectra, (Cambridge University Press, 1970).

11. B. Judd, Operator Techniques in Atomic Spectroscopy, (McGraw-Hill, New York, 1963).

12. B. N. Figgis, Introduction to Ligand Fields, (John Wiley, New York, 1966). 
$-24-$

13. M. 3. Weber, in Topics in Applied Physics 49 , Laser Spectroscopy of Solids, (Springer-Verlag, Berlin, 1981), p. 189.

14. G. F. Ambush and R. Kopelman, ibid., p. 1.

15. M. J. Weber, in Handbook of the Physics and Chemistry of Rare Earths, (North-Holland, New York, 1979), Vol. 4, p. 286.

16. W. Koechner, Solid State Laser Engineering, (Springer-Verlag, New York, 1976), p. 17.

17. H. F. Krupke, J. Quant. Electron. QE-10, 450 (1974).

18. A. E. Siegman, An Introduction to Laser and Masers, (McGraw-Hi11, New York, 1971), p. 110. 
$-25-$

Part II

Studies of Vibronic Spectra in Disordered Solids 
Chapter 3: VIBRONIC SPECTRA OF $\mathrm{Gd}^{3+}$ IN DISORDERED SOLIDS A. Introduction

A careful inspection of absorption or fluorescence spectra of . rare-earth ions in solids often reveals weak, broad, and temperature dependent features in addition to the purely electronic transitions predicted by the static crystal-field theory of Chapter 2. These side bands can extend hundreds of $\mathrm{cm}^{-1} \mathrm{~s}$ on both the high and low-frequency sides of the sharp lines between Stark states. They are a manifestation of a weak ion-phonon interaction and result from vibrationally-assistedelectronic or "vibronic" transitions. In a fluorescence transition, for example, an excited rare earth (RE) usualiy relaxes radiatively to a lower-energy state by emission of a single photon; it may also relax, with a much smaller probability, by simultaneous?y emitting a photon and creating a quantum of vibrational energy (a fhonon) in the host. Since some of the original electronic energy has gone into creating a vibration, the emitted photon is of lower energy (longer wavelength) than those from purely electronic or "zero-phonon" transitions and is said to have been Stokes shifted. The distribution of phonon energies in solids allows creation of photons with a variety of energies and one obtains broad bands in spectra. Simultaneous emission of a photon and absorption of a quantum of lattice energy is also possible, resulting in fluorescence photons of higher energy than the zero-phonon line; this is termed an anti-Stokes shift. Analogous processes occur during optical absorption by REs in solids. 
The effect of charged neighboring ions on RE energy states and transition probabilities was treated in Chapter 2 by inclusion of a crystal-field term in the perturbation Hamiltonian, assumed to be due to reighboring ions that were fixed in space. Ions in solids, of course, are not fixed but vibrate about their equilibrium positions and other terms that account for the dynamic character of the crystal field must be included for a description of vibronic transitions. A semi-classical and physical explanation of the origin of vibronic transitions is useful. When an oscillating radiation field is applied to the solid, it creates an electronic dipole moment at the RE, the strength of which is modulated by the time-dependent part of the crystal field at frequencies characteristic of the normal modes of vibration of the host. Modulation results in dipolar resonances (transitions) at frequencies which are a sum (Stokes shifted) and difference (anti-Stokes shifted) of the driving and modulating frequencies as well as at the-fundamental, driving (zero-phonon) frequency.

Vibronic transitions provide a direct, localized, and sensitive probe of the ion-phonon coupling between a RE and host which has great effect on the ion's optical properties. As discussec in Chapter 2, this interaction influences the lifetime and quantum efficiency of an ion's excited state since nonradiative relaxation from a metastable fluorescing state is via phonon emission. ${ }^{1}$ It usually determines the homogeneous linewidth of a transition by allowing rapid transitions to other close-lying electronic states, resulting in lifetime broadening. 
From another point of view, RE vibronic spectra provide information about vibrations of the host. By emphasizing the energy difference between the observed shifted photons and the zero-phonon.line, a vibronic spectrum is essentially a representation of the vibrational density of states about the RE, weighted by a transition probability that accounts for differences in coupling of phonons to the electronic transition. In a manner analagous to Raman scattering spectra, a RE vibronic spectrum may be thought of as a probe that shifts a representation of the low-frequency vibrational modes into the optical frequency range. B. Vibrations in Disordered Solids

Although vibrations of perfect crystals are well understood, ${ }^{2}$ those of disordered crystals ${ }^{3}$ and especially glasses ${ }^{4}$ are not; this is mainly a result of the lack of mathematical simplicity which is concomitant with the periodicity of perfect crystals. In the past decade, a structural model of insulating and semiconducting glasses has become well-established, the continuous random network (CRN) model which was first advanced by W. H. Zachariasen ${ }^{5}$ in 1932. In this model, ions in glass possess the short-range order that is apparent when considering bonding in crystals of similar composition but lack the long-range order of a periodic structure. For example, silicon ions in glassy $\mathrm{SiO}_{2}$ (fused silica) are tetrahedrally coordinated by four oxygen ions just as they are in all except one of the 22 crystalline polymorphs of $\mathrm{SiO}_{2}$ and in almost all silicate crystals. " These tetrahedral "building blocks" may be linked to form a network of bonds by making the corner oxygen ions common to two tetrahedra. The tetrahedra, need not have the specific 
relative orientation required of a crystal, however, and may be oriented in a random manner so that long-range order is lost. With a physically acceptable amount of bond stretching and bending, a closed, dense, and macroscopically amorphous structure may be obtained with properties consistent with results of structure sensitive experiments such as $x$-ray and elastic neutron scattering.

Conceptual models designed, to understand the dynamical properties of. the CRN have generally fallen into two extremes. One viewpoint examines the normal modes of small "molecular" units and chooses some method to. simulate the coupling to the other units that make up the glass. One boundary technique is the cluster-Bethe-lattice approach, ${ }^{7}$ in which the atoms of the molecular unit are assumed connected to a tree-like network of atoms with the nearest-neighbor geometry of the actual solid but without the topology ( $r$ ings of bonds) of a real three-dimensiona? structure; trees branching from different atoms of the central unit do not interconnect. A Green's function method may then be used to obtain spectra with structure attributed to the central unit and a broad background due to the boundary network. Another approach, appropriate for chain-like glasses (e.g. metaphosphates, metasilicates), is to envision units connected in a loop and assume one-dimensional periodic boundary conditions. ${ }^{8}$ These models, although physically insightful, do not have enough degrees of freedom to accurately predict all the features of Raman scattering and infrared absorption spectra.

The other extreme considers "macromolecular" units, assumes or fits interatomic force constants, and solves for vibrational eigenfrequencies 
and eigenvectors by brute-force matrix diagonalization ${ }^{9}$ or with clever numerical techniques. ${ }^{10}$ This approach was first applied by Bell, Dean, and co-workers, ${ }^{11}$ to a hand-built CRN model of $\mathrm{SiO}_{2}$ consisting of 503 atoms, Recently, models generated by molecular dynamic computer simulations have been examined ${ }^{12-14}$ and apparently predict vibrational spectra very well. A problem with the macromolecular approach is that motion of a large number of particles is difficult to comprehend and precludes an intuitive, physical understanding. Predictions of experimental results of a glass with slightly different composition requires that a new model be constructed and spectra recalculated.

Basically, workers are struggling to find the correct questions to ask to distill the "physics" out of large and small model results. Any complete picture must be able to predict such things as how frequencies and line-strengths change in spectra of glasses of different materials but with the same structure (e.g. $\mathrm{SiO}_{2}$ and $\mathrm{GeO}_{2}$ ), the effect of different modifier cations on vibrations, and also give a qualitative description of the motion of atoms in modes in different frequency ranges.

Vibronic spectra provide an independent vibrational spectroscopy that differs significantly from the more conventional spectroscopies (inelastic neutron, Raman, and infrared) in that it probes motion of ions on a very localized scale. All experimental techniques emphasize a specific subset of modes within the vibrational density of states, as determined by matrix element effects, and provide clues necessary to formulate the questions required for an understanding of glass vibrations. 
C. Previous Vibronic Studies

Vibronic sidebands of a RE in crystal were first identified by Ewald ${ }^{15}$ in absorption spectra of $\mathrm{Nd}^{3+}$ in $\mathrm{Zn}_{3} \mathrm{Nd}_{2}\left(\mathrm{NO}_{3}\right)_{12}$ and treated theoretically in terms of varying crystalline field by Van vleck. 16 The current theoretical interpretation was developed by Satten who initially treated modes of a molecular complex consisting of the RE ion and certain of its neighbors. 17 . When the discrete vibrational frequencies. of this model proved inadequate to explain the complexities of vibronic sidebands, interaction of the ion with lattice modes of the perfect crystal was considered. ${ }^{18}$ It was found that $k=0$ modes alone were insufficient to account for all details of structure but, when analys is included some high-symmetry directions of the Brillouin zone, ${ }^{19}$ there was reasonable agreement between calculation and experiment for the examined $\mathrm{Pr}^{3+}$ doped $\mathrm{LaCl}_{3}$ system. Often, sharp lines not predicted by pure-lattice models appear in vibronic spectra; 20 these are associated with "impurity" modes that result from the presence of the RE through either local modification of bonding force constants or the changes in structure necessary for charge compensation. Impurity modes have been treated in analys is of vibronic spectra by using Green's function techniques. 21

Studies of vibronic transitions in glass are complicated by the disordered nature of the host. As discussed in Chapter 2, this disorder causes inhomogeneous broadening of spectral features due to the distribution of perturbing environments experienced by ions in glass. Purely electronic, zero-phonon transitions of different classes of ion 
sites occur at different frequencies. In fluorescence with nonselective excitation, for example, vibronic transitions fron different sites are s'dperimposed into a broad, unresolved spectrum.

The only previously reported observations of vibronic sidebands in glass, 22,23 employed a rare earth $\left(\mathrm{Eu}^{3+}\right)$ as the probe ion. Trivalent europium has a relatively simple energy level scheme and, for $4 f-4 f$ transitions, the ion-phonon coupling is weak. Vibronic transitions were detected in the ${ }^{7} F_{0} \rightarrow{ }^{5} D_{0}$ excitation spectrum of the strong $5_{D_{0}}-7_{F_{2}}$ emission. Zakharov et al. ${ }^{22}$ found that they could. correlate many of the observed vibronic bands with bands in infrared reflection spectra, but in a number of cases, vibronic frequencies could not be correlated with any infrared frequency. In these nonresonant studies, the vibronic spectra were subject to the same inhomogeneous broadening as the zero-phonon line.

Selective excitation of ions in glass with the same transition frequency and, perhaps, similar local environments is possible using techniques of laser-induced fluorescence line narrowing. ${ }^{24}$ we have used this technique to circumvent inhomogeneous broadening and reveal true vibronic band widths of a subset of selected ions.

D. Vibronics of $\mathrm{Gd}^{3+}$

In all of our experiments, trivalent gadolinium as a dilute impurity was selected for the probe ion. An energy level diagram of the relevant states of $\mathrm{Gd}^{3+}$ in glass is shown schematically in Figure 1. Excitation is possible via the ${ }^{8} \mathrm{~S}_{7 / 2} \rightarrow{ }^{6} \mathrm{P}_{7 / 2}$ or ${ }^{8} \mathrm{~S}_{7 / 2} \rightarrow{ }^{6} \mathrm{P}_{5 / 2}$ transitions and fluorescence at room temperature or below occurs predominant l.y from the ${ }^{6} \mathrm{P}_{7 / 2}$ level. Energies refer to typical center of gravity energies of these levels in glass. This ion is well suited for vibronic studies 


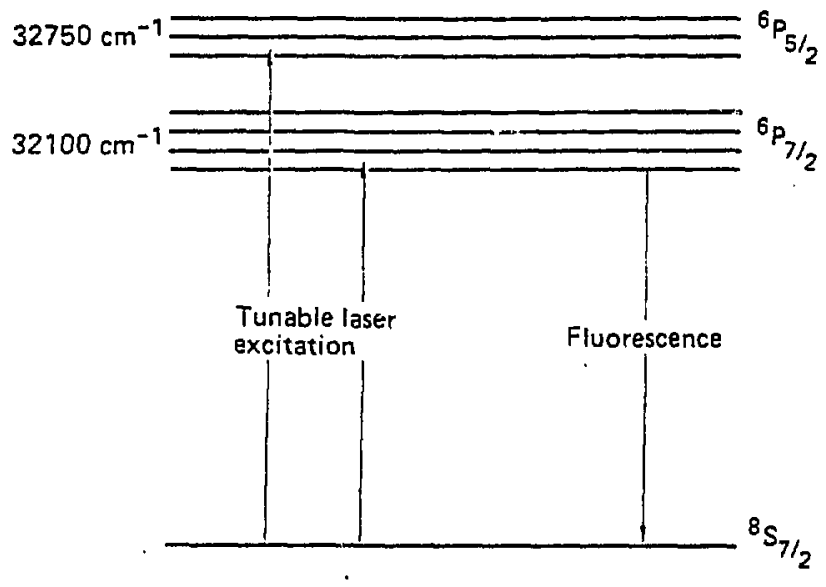

Figure 1: 'Schematic energy-level diagram of relevant states of $\mathrm{Gd}^{3+}$ ir a low-symmetry crystal field. 
because its ground levei is an $S$ state $(L=0)$ which experiences negligible splitting in any perturbing environment. Thus there is no confusion from overlapping vibronic transitions and zero-phonon transitions to different Stark states of the terminal J-level. In addition, there are no other J-levels within a single phonon energy of the terminal level to obscure the weak vibronic signal. Gadolinium transitions occur in the near ultra-violet, at frequencies where laser light sources and detectors are readily available.

To obtain true vibronic peak widths, liıe narrowing must be complete. If not, vibronic spectra are a convolution of shifted sidebands due to ions in different environments. Since the homogeneous linewidth of purely electronic transitions of REs in glass has been observed ${ }^{25}$ to vary as $T^{2}$, site selectivity and line narrowing are enhanced at low temperatures. Low sample temperatures alsn simplify spectra because only the lowest energy states of the excited ${ }^{6} P_{7 / 2}$ manifold will be populated. In addition, for optimal line narrowing, excitation should be limited to the low-frequency tail of the inhomogeneously broadened ${ }^{8} S_{7 / 2} \rightarrow{ }^{6} P_{7 / 2}$ absorption band. This rerluces the possibility of accidental coincidences of excitation bands. As discussed in Chapter 2, an accidental coincidence occurs, for example, when the lowest energy level of the ${ }^{6} P_{7 / 2}$ manifold of one ion is equal in energy to another ${ }^{6} \mathrm{P}_{7 / 2}$ level of an ion in a different local environment. The same excitation frequency results in resonant and nonresonant zero-phonon fluorescence transitions of different energies anc therefore a superposition of vibronic side bands. 
E. Glass Studies

The major goals of our giass vibronic studies were to answer the following two questions: (1) Are vibronics cheracteristic of vibrations of the entire glass network or impurity modes created by the presence of the RE? (2) Is there a site-to-site variation in the strength and distribution of the vibronic structure?

To answer the first question; a series of metaphosphate glasses was chosen as host because their structure has been studied and portions of their vibrational spectra have been characterized in terms of the motions of simple structural unit. ${ }^{26-20}$ This characterization is not rigorously established, however. Metaphosphate glasses have the sto ichiometry $M\left(\mathrm{PO}_{3}\right)_{x}$, where $M$ represents a metal modifier cation and $x$ is the oxidation state of the metal. Their structure is considered to be basically the same regardless of $M$ and consiscs of $\mathrm{PO}_{4}$ tetrahedra connected at two corners by "bridging" oxygens, forming polymeric chains. Modifier cations and RE dopants occupy sites between the non-bridging oxygen atoms of the tetrahedra and provide weak linkage between different chains.

As metal modifier cations we chose (1) $\mathrm{La}^{3+}$, an ion similar to $\mathrm{Gd}^{3+}$ in mass and charge, and (2) $\mathrm{Al}^{3+}, \mathrm{Ba}^{2+}, \mathrm{Mg}^{2+}$, ions which differ from $\mathrm{Gd}^{3+}$ in mass and/or charge. Thus we studied cases where the RE probe ion constituted a negligible perturbation as well as cases where it was both a mass and charge defect.

To obtain informatira about the vibrations of the unperturbed glass, polarized Raman scattering spectra and near-normal infrared reflectance 
were recorded for all of the glasses. These traditional vibrational spectroscopies obey different selection rules and therefore emphasize different vibrational modes. For the low RE concentrations of our sanples, thase vibrational spectra are characteristic of the undoped bulk giass. Because of dispersion associated with infrared reflection, the quantity most indicative of IR-active vibrational modes is the imaginary part of the dielectric constant, $\epsilon_{2}$. This was obtained numerically from reflectance data using a Kramers-Kronig transformation.

The second question was motivated by observed variations in zerophonon transition frequency and radiative and nonradiative transition probability of REs in glass ${ }^{24}$ attributed to differences in local environment. By varying the exciting laser wavelength in an FLN experiment, we may easily select a different subset of ions. If vibronic spectra are sensitive to differences in local structure, they may be used to determine or verify that structure just as other vibrational spectroscopies have been used fur structural studies of undoped glasses. ${ }^{8}$ To explore this question, we chose the simple glass former Bef $_{2}$ as host glass. Possible local structures of this glass about RE dopants are known from computer simulations 29 and its neutron, $I R$, and Raman spectra $30-32$ have been extensively studied. F. Crystal Studies

The FLN technique, developed to study REs in glass hosts which have a quasicontinum of local RE environments, may be used to explore zero-phonon and vibronic transitions of doped crystals in which a discrete number of site types exist. The different sites result when a trivalent RE is 
incorporated substitutionally into a lattice site normally occupied by a host ion of different ionic charge. An example of this occurs in the crystalline system described in Chapter 6, Gd:CaF 2 , in which $\mathrm{Gd}^{3+}$ replaces a doubly-charged $\mathrm{Ca}$ ion of the fluorite lattice. The extra charge of the RE provides an opportunity for the free energy of the lattice to be lowered by a variety of local structural changes; each distinct change may be looked at as a mechanism for compensation of the extra charge of the dopant ion. Three identified mechanisms are inclusion of an interstitial $F^{-}$ion in a neighboring unit cell, $F^{-}$ inclusion several cells away from the $R \mathrm{k}$, and substitution of a neighboring lattice fluorine by a doubly ionized impurity oxygen. In fact, Makovsky ${ }^{33}$ has identified eight different $\mathrm{Gd}^{3+}$ sites in $\mathrm{CaF}_{2}$ and found evidence for at least five others.

Each site type produces a different crystal fielo which interacts with the RE, creating a great number of transition frequencies. Absorption and broadband-excited fluorescence spectra are a convolution of contributions from different sites. Makovsky ${ }^{33,34}$ and others 35,36 have used standard optical methods in a partially successful attempt to unravel the energy levels schemes of the differently compensated sites of $\mathrm{Gd}^{3+}$ in crystals of the fluorite structure. These investigations are often supplemented by electron paramagnetic studies 37,38 of Gd and other REs which provide information of site symmetry by detection of ground state splitting.

Wright and coworkers ${ }^{39-41}$ have used a tunable laser light source to obtain excitation spectra of $\mathrm{Er}^{3+}$ in several alkaline earth fluoride 
crystals. Not only were energy levels unambiguously revealed, they were also able to investigate multiphonon and energy transfer relaxation of the individual sites. Their work ${ }^{39}$ confirmed the existence of clusters of RE ions, in addition to the isolated centers, at doping levels greater than 0.05 mole $\%$. They also report ${ }^{41}$ vibronic transitions associated with the ${ }^{4} \mathrm{I}_{15 / 2} \rightarrow{ }^{4} \mathrm{~S}_{3 / 2}$ excitation spectrum of $\mathrm{Er}^{3+}$ in one site type of $\mathrm{BaF}_{2}$.

Although vibronic spectra of $4 f^{n} \rightarrow 4 f^{n-1} 5 d$ transitions of $\mathrm{Gd}^{3+}$ and other RES in crystals are well documented, 42,43 those of intraconfigurational $4 f^{n}$ transitions are not. In the present work, FLN techniques have been used to explore vibrations about $\mathrm{Gd}^{3+}$ in several site types via vibronic transitions.

\section{G. Dynamic Crystal Field}

The crystal field is a function of the coordinates of the host ions that surround the RE. This dependence may be made explicit by a Taylor series expansion of $V$ in the solid's normal mode coord nates, $\sigma_{1}$, which implicitly contain the masses of the ions ${ }^{44}$ :

$$
\begin{aligned}
& v=v_{0}+\sum_{1} \frac{\partial V}{\partial q_{1} q_{1}}+\frac{1}{2} \sum_{i m} \frac{\partial^{2} V}{\partial q_{1} \partial q_{m}} q_{1} q_{m}+\ldots \\
& =v_{0}+\sum v_{1} q_{1}+\sum_{1 m} v^{\prime \prime}{ }_{1 m} q_{1} q_{m}+\ldots
\end{aligned}
$$

The constant term, $V_{0}$, is the static crystal field of Chapter 2 ; all coefficients of Eq. (1) may be expanded in terms of tensor operators in a manner analogous to Eq. (2-4). 
To treat the ion-phonon interaction, our Hamiltonian must include the contribution of the phonon field:

$$
\begin{aligned}
H & =H_{c f}+H_{e e}+H_{s o}+V_{0}+\frac{1}{2} \sum_{T}\left(\dot{q}_{1}^{2}+w_{1}^{2} q_{1}^{2}\right)+\sum_{1} V_{j} q_{1}+\sum_{i m} V_{i m}^{\prime \prime} q_{1} q_{m}+\cdots \\
& =H_{\text {ion-static }}+H_{\text {phonon }}+H_{\text {ion-phonon }}
\end{aligned}
$$

We assume $H_{i o n-p h o n o n}$ is small and treat it as a perturbation. The unperturbed eigenfunctions of $\mathrm{H}_{\text {ion-static }}+\mathrm{H}_{\text {phonon }}$ are product wavefunctions and may be represented in Dirac notation as

$$
[\psi\rangle=\left[\phi_{i}\right\rangle\left[n_{1}, n_{2}, \cdots \cdot\right\rangle
$$

where $\left[\phi_{j}\right\rangle$ is the electronic Stark state of the ion and $\left[n_{1}, n_{2} \ldots\right\rangle$ is the occupation number representation of the lattice vibrational state (i.e. $n_{1}$ is the number of phonons in normal mode 1 ).

The normal coordinate of the $1^{\text {th }}$ mode may be expressed in terms of the annihilation and creation operators of phonons, ${ }^{45} b_{1}$ and $b_{1}^{+}$; i.e.

$$
a_{1}=c_{1}\left(b_{1}+b_{1}^{+}\right)
$$

with

$$
c_{1}=\left[\frac{\hbar}{2 \omega_{1}}\right]^{\frac{1}{2}}
$$


This form makes evaluation of matrix elements of $H_{i o n-p h o n o n}$ simple. For example, we may quickly obtain the temperature dependence of the emission of a photon and creation of a phonon in the $m^{\text {th }}$ normal mode (i.e. a Stokes-shifted vibronic fluorescence transition). The initial state may be represented as $\left[\phi_{i}\right\rangle\left[n_{1}, \ldots n_{m}, \ldots\right\rangle$ and the final state by $\left[\phi_{f}\right\rangle\left[n_{1}, \ldots n_{m+1}, \ldots.\right\rangle$ The rate of this transition, $P$, is proportional to the square of the matrix element of $\mathrm{H}_{j o n-p h o n o n}$ by Fermi's Golden Rule. ${ }^{46}$ Keeping only the first term in $H_{\text {ion-phonon' }}$ we find

$$
\begin{aligned}
P & \propto\left[\left\langle\phi_{f}\right]\left\langle n_{1}, \ldots n_{m+1}, \ldots\right] \sum_{1} v_{j} c_{1}(b,+b+1)\left[\phi_{i}\right\rangle\left[n_{1}, \ldots n_{m}, \ldots\right\rangle\right]^{2} \\
& =\left[c_{m}\left\langle\phi_{f}\right] v_{m}^{1}\left[\phi_{i}\right\rangle\left\langle n_{m}+1\right] b_{m}^{+}\left[n_{m}\right\rangle\right]^{2}
\end{aligned}
$$

since $V^{\prime}{ }_{1}$ is only a function of the RE's electrons coordinates and $b_{1}^{+}$only connects those phonon states with $n^{\prime},=n_{1}+1$.

Thus,

$$
P \propto\left[c_{m}\left\langle\phi_{f}\right] v_{m}^{\prime}\left[\phi_{i}\right\rangle\right]^{2}\left(n_{m}+1\right) .
$$

The temperature dependence of the process is contained entirely in the phonon occupation number, $n_{m}$; i.e.

$$
n_{m}=\frac{1}{\exp \left(\hbar \omega_{m} / k T\right)-1}
$$


As $\mathrm{T} \rightarrow 0, \mathrm{n}_{1} \rightarrow 0$ for all modes, but the transition is still possible by spontaneous phonon emission. Anti-Stokes emission is found to be proportional to $n_{m}$ (as opposed to $n_{m}+1$ ) and "freezes out" at low temperatures.

Examination of the electronic portion of the matrix element in Eq. (6) shows that transitions via an electric-dipole process, which may be forbidden by the symmetry of the static crystal field, can become allowed in vibronic transitions if $V_{m}^{\prime}$ has terms of odd-order in a tensor operator expansion.

Higher-order terms in the expansion of $H_{i o n-p h o n o n}$ correspond to multiphonon processes; ${ }^{47}$ for REs, these terms are very much smaller than the first-order term and two and three phonon sidebands are rarely observed. The ion-phonon interaction in the first-order approximation explains a variety of observed temperature dependent phenomenon such as homogeneous linewidths and a slight frequency shift of zero-phonon transitions.

It should again be emphasized that REs have been treated within the weak-field approximation of crystal field theory. All electronic states of the $4 f^{n}$ configuration have approximately the same bonding to ions of the host so that the lattice may be described by the same phonon. states irrespective of the electronic state of the ion. Other models, ${ }^{48}$ such as the configuration coordinate model, are more appropriate for ions whose electrons interact more strongly with the host. Optical transitions of these ions may be completely dominated by vibronic transitions, except at temperatures approaching $T=0$. 


\section{References}

1. B. DiBartolo, Optical Interactions in Solids, (John Wiley, New York, 1968) Ch. 15.

2. See, e.g. M. Born and K. Huang, Dynamical Theory of Crystal Lattices (Oxford University Press, London, 1954).

3. A. S. Barker and A. J. Sievers, Rev. Mod. Phys. 47, Suppl. No. 2, (Fa)11 1975).

4. D. Weaire and P. C. Taylor, "Vibrational Properties of Amorphous Solids", in Dynamica) Properties of Solids, 4 Ch. 1 (North-Holland, 1980).

5. W. H. Zachariasen, J. Am. Chem. Soc. 54, 3841 (1932).

6. R. B. Sosman, The Phases of Silica, (Rutgers University Press, New Jersey, 1965) p. 284; the number of known phases may have increased since publication of this book but any new phase would undoubtedly be a high-temperature and high-pressure polymorph.

7. R. B. Laughl in and J. D. Joannopoulos, Phys. Rev. B 162942 (1977).

8. S. Brawer, Phys. Rev. B ? 1173 (1975).

9. P. Dean, Rev. Mod. Phys. 44127 (1972).

10. S. R. Nage 1, A. Rahman, and G. Grest, Phys. Rev. Lett. 471665 (1981).

11. R. J. Bell and P. Dean, Nature 2121354 (1966); R. J. Bell, N. F. Bird, and P. Dean, J. Phys. C: Solid St. Phys, 1299 (1968); R. J. Bell, P. Dean, and D. C. Hibbins-Butler, J. Phys. C.: Solid St. Phys. $\underline{3} 2111$ (1970).

12. A. Rahman, M. J. Mandel1, and J. P. McTague, J. Chem. Phys. 641564 (1976).

13. S: H. Garofalini, J. Chem. Phys. 763189 (1982). 
14. S. A. Brawer, (private communication).

15. H. Ewald, Ann. Physik 34209 (1939).

16. J. H. Van Vleck, J. Chem. Phys. Z, 72 (1939).

17. R. A. Satten, J. Chem. Phys. 27286 (1957); R. A. Satten, J. Chem. Phys. 29, 658 (1958).

18. I. Richman, R. A. Satten, and E. Y. Wong, J. Chem. Phys. 391833 (1963).

19. R. A. Satten, J. Chem. Phys. 401200 (1961).

20. e.g. T. Timusk and M. Buchanan, Phys. Rev., 164345 (1967).

21. M. Buchanan and E. J. Woll, Can. J. Phys. 471757 (1969).

22. V. K. Zakharov, I. V. Kovaleva, V. P. Kolobkov, and L. F. Nikolaev, Opt. Spectrosc. (USSR) 42, 532 (1977).

23. V. P. Lebedev and A. K. Przhevuskii, Opt. Spectrosc. (USSR) 48, 513 (1980).

24. M. J. Weber, Laser Excited Fluorescence Spectroscopy in Glass, in Laser Spectroscopy of Solids, ed. W. M. Yen and P. M. Selzer (Springer-Verlag, Berlin, 1981) p. 189.

25. J. Hegarty and W. M. Yen, Phys. Rev. Lett. 43, 1126 (1979).

26. Ya. S. Bobovich, 0pt. Spectrosc. (USSR) 13, 274 (1962).

27. G. B. Rouse, P. J. Miller, W. M. Risen, J. Non-Cryst. Solids 28, 193 (1978).

28. B. N. Nelson and G. J. Exarhos, J. Chem. Phys. 71, 2739 (1979).

29. S. A. Brawer and M. J. Weber, J. Non-Cryst. Solids 38/39 9 (1980).

30. F. L. Galeener, A. J. Leadbetter, and M. W. Stringfellow (to be published).

31. G. E. Wairafen and R. H. Stolen, Solid State Commun. 21, 417 (1977). 
32. F. L. Galeener, G. Lucovsky, and R. H. aeils, Solid State Commun. 25, 405 (1978).

33. J. Makovsky, in Physics of Quantum Electronics, ed. P. L. Kelly, B. Lax, and P. E. Tannenwa 1d, (McGraw-Hil1, New York, 1965), p. 340. 34. J. Makovsky, J. Chem. Phys. 46390 (1967).

35. F. 2. Gilfanov, L. D. Livanova, and A. L. Stolov, Sov. Phys. Solid State 8108 (1966).

36. H. M. Crosswhite, R. L. Schwiesow, and W. T. Carnail, J. Chem. Phys. $505032(1969)$.

37. J. Sierro, Phys. Lett. 4178 (1963).

38. M. J. Weber and R. W. Bierig, Phys. Rev. 134 A 1492 (1964).

39. J. C. Wright, in Spectroscopie des Elements de Transition et des Elements Lourds dons les Solides p. 211.

40. D. R. Tallant and J. C. Wright, J. Chem. Phys. 632074 (1975).

41. M. P. Miller and J. C. Wright, J. Chem. Phys. 71324 (1978).

42. M. Schlesinger and G. W. F. Drake, Can. J. Phys. 541699 (1976).

43. D. L. Hood and W. Kaiser, Phys. Rev, 1262079 (1962).

44. A. A. Maradudin, E. W. Montroll, and G. H. Weiss, Solid State Physics, Supplement 3, (Academic Press, New York, 1963).

45. H. Haken, Quantum Field Theory of Solids, (North-Holland, New York, 1976).

46. L. I. Schiff, Quantum Mechanics, 3rd ed. (McGraw-Hi11, New York, 1968), p. 285.

47. W. M. Yen, W. C. Scott, and A. L. Schawlow, Phys. Rev. 136A, 271 (1964).

48. G. F. Imbush and R. Kopelman in Laser Spectroscopy of Solids, W. M. Yen and P. M. Selzer, eds. (Springer-Verlag, Berlin, 1981). 
Chapter 4: EXPERIMENTAL TECHNIQUE

Experimental details of the Gd vibronic studies are now presented. Sample properties and apparatus of the fluorescence line narrowing (FLN), Raman scattering, and infrared reflectance experiments are discussed.

\section{A. Samples}

The following four metaphosphate glasses were investigated (compositions in mole $\%$ ):

$50 \mathrm{P}_{2} \mathrm{O}_{5} \circ 49 \mathrm{MO} 1 \mathrm{Gd}_{2} \mathrm{O}_{3}$, where $M=M_{9}, \mathrm{Ba}$ and

$75 \mathrm{P}_{2} \mathrm{O}_{5} \circ 24 \mathrm{M}_{2} \mathrm{O}_{3} \circ \mathrm{Gd}_{2} \mathrm{O}_{3}$, where $M=A 1$, La.

Samples were prepared by the Inorganic Glass Section of the National Bureau of Standards from reagent-grade materials using normal melting and casting methods. Typical size of polished samples was $10 \times 10 \times 5 \mathrm{~mm}$. They were of good optical quality with few bubbles or striations..

Beryllium fluoride glass with 0.5 mole $\% \mathrm{GdF}_{3}$, doping was melted and prepared at LLNL with care commensurate with the toxicity of beryllium compounds. Since Bef 2 is hygroscopic, samples were stored in a desicator under a dry-nitrogen atmosphere. Sample surfaces degraded slightly after several months, increasing the amount of scattered light in FLN experiments.

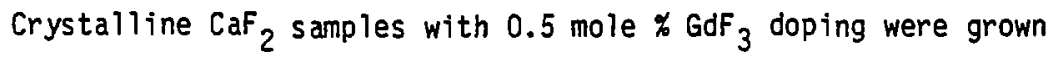
by Optovac, Inc.; using the Bridgman-Stockbarger method with "gettering" techniques used to prevent incorporation of oxygen or hydrogen into the lattice. Unoriented polished samples had typical dimensions of $10 \times 5 \times$ $5 \mathrm{~mm}$. Crystals had some inclusions and striations but light scattering was relatively small. 


\section{B. Fluorescence Line Narrowing}

A schematic diagram of the apparatus used for FLN measurements of all samples is shown in Figure 1. Gadolinium ions were excited into the ${ }^{6} \mathrm{P}_{7 / 2}$ band with a frequency-doubled, flashlamp-pumped dye laser (Chromatix CMX-4). A solution of rhodamine $6 G$ with $4 \%$ Ammonyx $L O$ in water provided a 1 asing medium with a fundamental tuning range from $\simeq 610$ to $630 \mathrm{~nm}$ and with good durability to prolonged operation. The laser was operated at $10 \mathrm{~Hz}$ producing $\simeq 3.0 \mathrm{~mJ}$ pulses at $624 \mathrm{~nm}$ of $\simeq 1 \mu \mathrm{s}$ duration. The fundamental beam was frequency-doubled by an intracavity ammonium dinydrogen phosphate (ADP) crystal, resulting in $\simeq 0.2 \mathrm{~mJ}$ of $312 \mathrm{~nm}$ light with a spectral width $<3 \mathrm{~cm}^{-1}$. Residual fundamental light was removed by a UV-pass/visible block filter (Corning 7-54). The beam was directed into a dark box enclosure surrounding the sample by aluminum front-surfaced mirrors and focused at the sample by a 1-m focal length lens. (The apparatus configuration is very similar to that depicted in more detail in Figures 3 and 4 of Chapter 8 ). A portion of the beam was reflected by a fused silica beam splitter onto the surface of a UV-photodiode (Hi-voltage Components, POH-114-P) that was used to monitor the energy stability of the laser output. The signal from this diode was processeu by a boxcar averager (PAR, model 162) with its gate set to integrate the input over the entire pulse duration. This provided a OC voltage proportional to the average energy of a given number of laser pulses.

Samples were mounted in a dewar with fused silica windows and cooled to $\simeq 20 \mathrm{~K}$ by a closed cycle hel jum fluid refrigerator (Air Products, Displex 202-5). A right-angle excitation/observation geometry was 


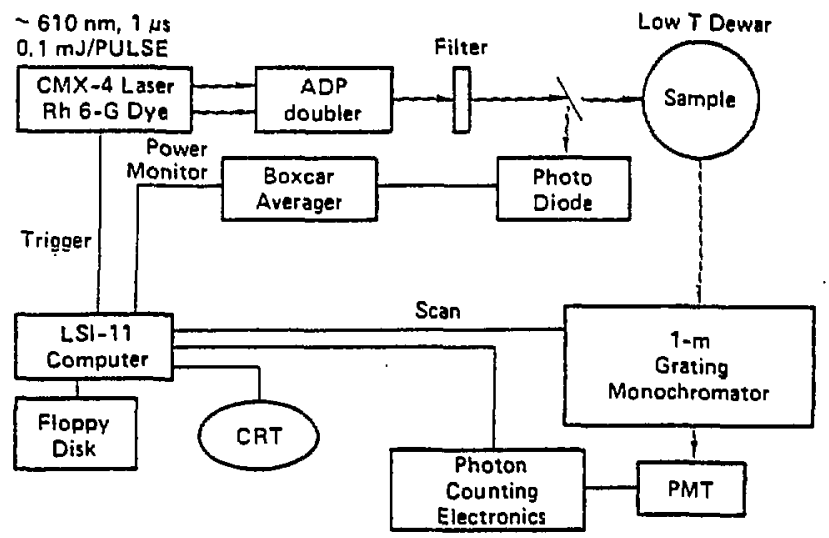

Figure 1: Schematic diagram of fluorescence line narrowing apparatus. 
employed in which ihe exciting laser beam was directed vertically through the botton of the sample and reflected back along the same path by a mirror mounted on the top of the sample holder; this prevented problems; of heat-loading of the refrigerator by the beam and also increased the probability of $\mathrm{Gd}^{3+}$ excitation.

The vertical fluorescence streak along the path of the laser bean provided optimal imaging onto the vertical slits of a 1-in spectrometer (Chromatix CT-103, blaze $=0.5 \mu \mathrm{r} 1200$ lines/cm). Imaging was accomplished using a collection lens $(f l=7 \mathrm{~cm}, f / 1.5)$ and a lens $(f)=$ $15 \mathrm{~cm}, \mathrm{f} / 7)$ consistent with the spectrometer's effective speed. 'A polarization scrambler was placed between the final lens and the entrance slit of the spectrometer to eliminate possible polarization bias. The spectrometer was typically operated with entrance and exit slit widths of $300 \mu \mathrm{m}$ for vibronic spectra esulting in s? it width limited resolution of $<30 \mathrm{~cm}^{-1}$. The relatively large slit widths were necessary as a result of the low intensity of vibronic sidebands. Spectral accuracy of the apparatus was $\leq 5 \mathrm{~cm}^{-1}$ and $\mathrm{s} l$ it height was $1 \mathrm{~cm}$.

Fluorescence was detected by an ambient temperutu e S-20 photomultiplier (Amperex. PM2233A) in a gated photon-counti.ng configuration. The photo..-counting amplifier/discriminator (PAR model. 1121) was operated with a discriminator threshold of $2.5 \mathrm{mV}$, which gave an optimum signal/noise vs. signal throughput value at a PMT bias of 2.1 kV. This threshold resulted in a dark count of $\simeq 500 \mathrm{~s}^{-1}$. A pulse conditioning operational amplifier converted the output of the amplifier/discriminator to TTL compatible levels. 
Photon counting of fluorescence for longer than a few decay time constants $\left(=3-10 \mathrm{~ms}\right.$ for ${ }^{6} \mathrm{P}_{7 / 2}$ transitions of $\mathrm{Gd}^{3+\cdot}$ in solids) is not useful since after this time the signal is small and counts are mostly from noise. A photon-counting gate, which limits the collection time of signal pulses, was included by applying the conditioned det.ctor signal and that of a delay/pulse generator (Tektronix 2101) to an AND logic circuit. Pulses from the detector were passed by the circuit only when the pulse generator voltage was high (logic level 1); the gate also allowed examination of fluorescence signals at fixed time delays from the exciting laser pulse. This feature was used to test for energy transfer between different RE sites by comparing line-narrowed zero-phonon spectra at zero delay and at $7 \mathrm{~ms}$ delay using a $1 \mathrm{~ms}$ gate. No detectable thanges in spectral shape were observed, indicating that at doping levels of our samples, negligible transfer occurred. Signal pulses from the gate were counted and stored by a multichannel scaler, or MCS, (Tracor Northern NS-570).

Timing and control signals necessary for acquisition of a spectrum were generated by an LSI-II microcomputer. The computer fired the laser a given number of times for one wavelength data point (typically 100 shous). It also simultaneously triggered the photon-counting gate genrator and the boxcar averager. After the laser shots, it would read (via an analog-to-digital converter), store, and zero the DC signal of the boxcar averager which was proportional to the total energy impinging on the sample for that data point. Then it advanced the spectrometer, incremented the channel of the MCS, and fired the laser for the next data 
point. After an entire spectrum was collected, data was transferred from the MCS to an array in the computer. The previously nieasured dark count was subtracted and an energy normalized spectrum obtained. This was displayed in various formats on a graphics terminal (Tektronix 4010), and stored on floppy disk. The computer also allowed averaging of several runs for improved signal to noise.

\section{Raman Spectra}

Polarjzed Raman scattering spectra of the metaphosphate glasses were obtained at room temperature using either the 488.0 or the $514.5 \mathrm{~nm}$ line of an argon ion laser (Spectra Physics nodel 165). A $90^{\circ}$ scattering geometry was employed incorporating film polarizer, polarization scrambler, and a double monochromator (SPEX model 1401) as analyzer. The detection system consisted of a cooled RCA $31034 \mathrm{~A}$ phototube in a photon-counting mode. Counting rates were typically $5 \times 10^{4} \mathrm{~s}^{-1}$. Raman spectra were not corrected for the increased population of law-frequency phonon modes at room temperature. Resolution of the apparatus was $<5 \mathrm{~cm}^{-1}$. Data collection, storage and display was controlled by a microcomputer (Tektronix 4051). The Raman system is described in greater detail in Reference 1.

\section{Infrared Reflection Spectra}

Room temperature infrared refleciance of the metaphosphate glasses was measured at $15^{\circ}$ from normal using a Fourier transform spectrometer (DIGILAB FTS14). A useful discussion of transform spectroscopy is found in Reference 2. Data from $170-400 \mathrm{~cm}^{-1}$ were recorded using a mylar beamsplitter and from $400-4000 \mathrm{~cm}^{-1}$ using Ge on $\mathrm{KBr}$. A front-surface gold mirror was used as reference and assumed to be $100 \%$ reflecting in the region of measurement. 
$-51-$

References

1. D. L. Perry, S. M.. Klainer, H. R. Rowman, F. P. Milanovich, T. Hirschfeld, and S. Miller, Anal. Chem. 53, 1048 (1981).

2. H. W. Siesler, Proc. of 5th European Symp. on Polymer Spectroscopy, ed. D. O. Hummel (Verlag, Berlin, 1979), p. 137. 
Chapter 5: GLASS RESULTS AND DISCUSSION

A. Metaphosphate Glass Experimental Results

Laser excited resonant ${ }^{6} \mathrm{P}_{7 / 2} \rightarrow{ }^{8} \mathrm{~S}_{7 / 2}$ fluorescence of

$\mathrm{Ba}\left(\mathrm{PO}_{3}\right)_{2}: \mathrm{Gd}^{3+}$ at $293 \mathrm{~K}$ and $20 \mathrm{~K}$ is shown in Figure 1. At low

temperatures the homogeneous linewidth is reduced, which greatly improves

the line narrowing and increases the site selectivity. At $293 \mathrm{~K}$, weak

Stokes-shifted vibronic sidebands are observed extending out to $\simeq 325$

$\mathrm{nm}$. The peak vibronic amplitude is only $1 / 50$ th the magnitude of the

zero-phonon line. A search was made for two-phonon vibronic sidebands

but none were detected; this placed an upper limit on their intensity of

$<10^{-3}$ of the zero-phonon intensity. The weak fluorescence band in

Figure 1 at $\simeq 307 \mathrm{~nm}$ is ${ }^{6} \mathrm{P}_{5 / 2} \rightarrow{ }^{8} \mathrm{~S}_{7 / 2}$ emission. The first

e-folding times of the ${ }^{6} P_{7 / 2}$ state for these glasses ranged from 3-5 ms and were independent of temperature.

Vibronic spectra for the metaphosphate glass series are shown in

Figures 2-5. The abscissa represents the frequency shift of the phonon-assisted fluorescence from the peak of the line-narrowed zero-phonon line. The large increase in the vibronic intensity at low frequency does not imply a large density of states but is the result of a superposition of vibronic signal and the tail of the much more intense zero-phonon line (observed with a spectral slitwidth of $30 \mathrm{~cm}^{-1}$ ).

Also plotted in Figures 2-5 are $H H$ and HV Raman spectra. The notations $H H$ and HV indicate spectra recorded with the polarization of the incident and scattered radiation parallel or perpendicular to one another, respectively. 


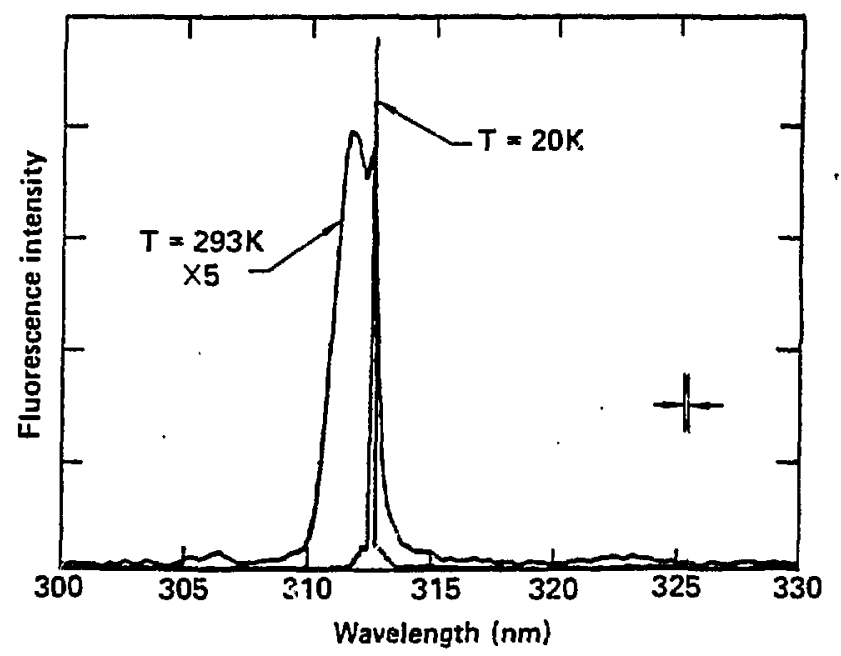

Figure 1: Laser-excited ${ }^{6} \mathrm{P}_{7 / 2} \rightarrow{ }^{8} \mathrm{~S}_{7 / 2}$ fluorescence of $\mathrm{Gd}^{3+}$ in $\mathrm{Ba}\left(\mathrm{PO}_{3}\right)_{2}$ glass. Excitation wavelength: $312.5 \mathrm{~nm}$. 

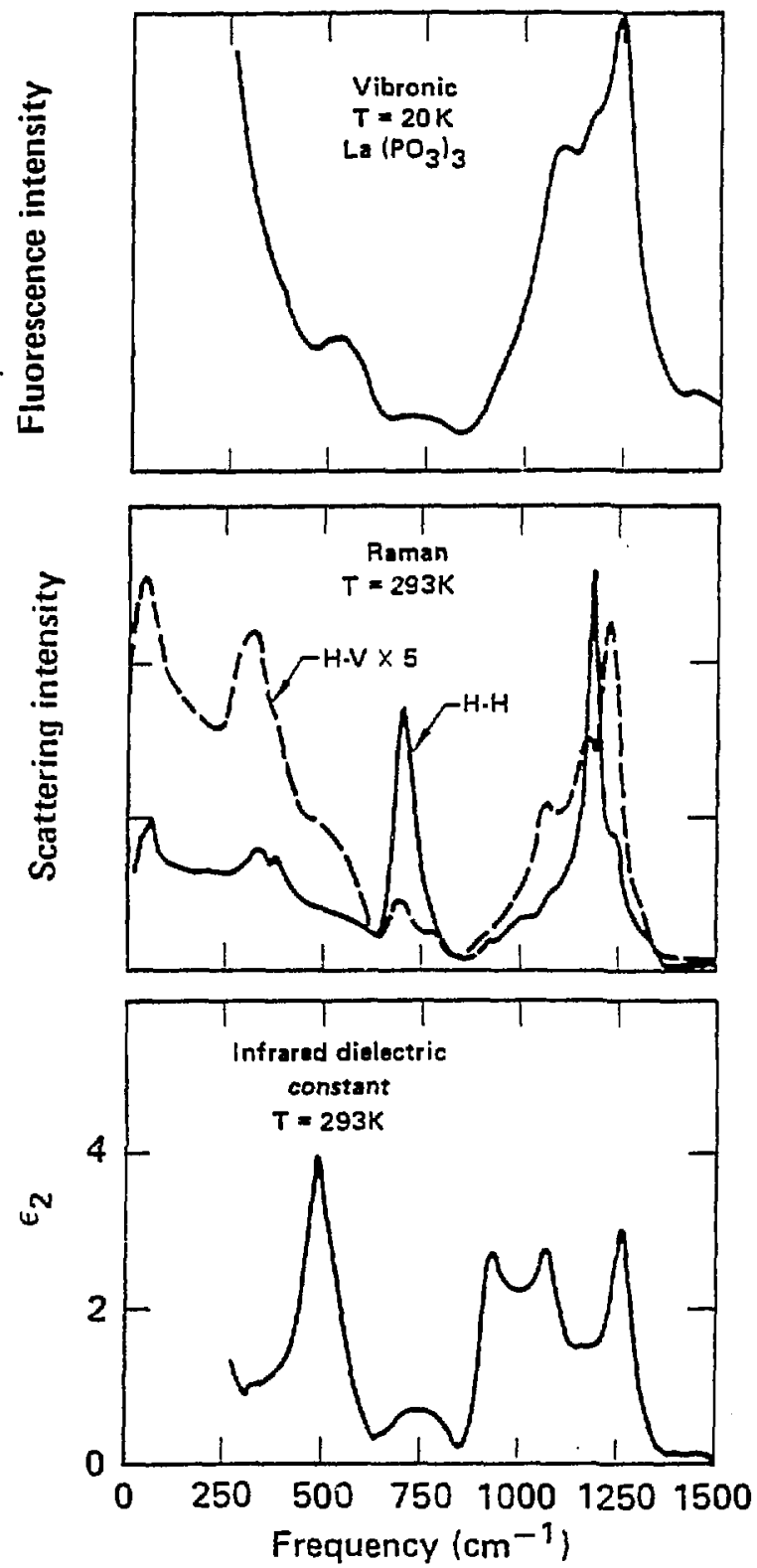

Figure 2: Comparison of the vibronic, $H H$ and HV polarized Raman, and infrared (dielectric constant) spectra of $\mathrm{La}\left(\mathrm{PO}_{3}\right)_{3}$ : Gd $\mathrm{g}$ lass. 

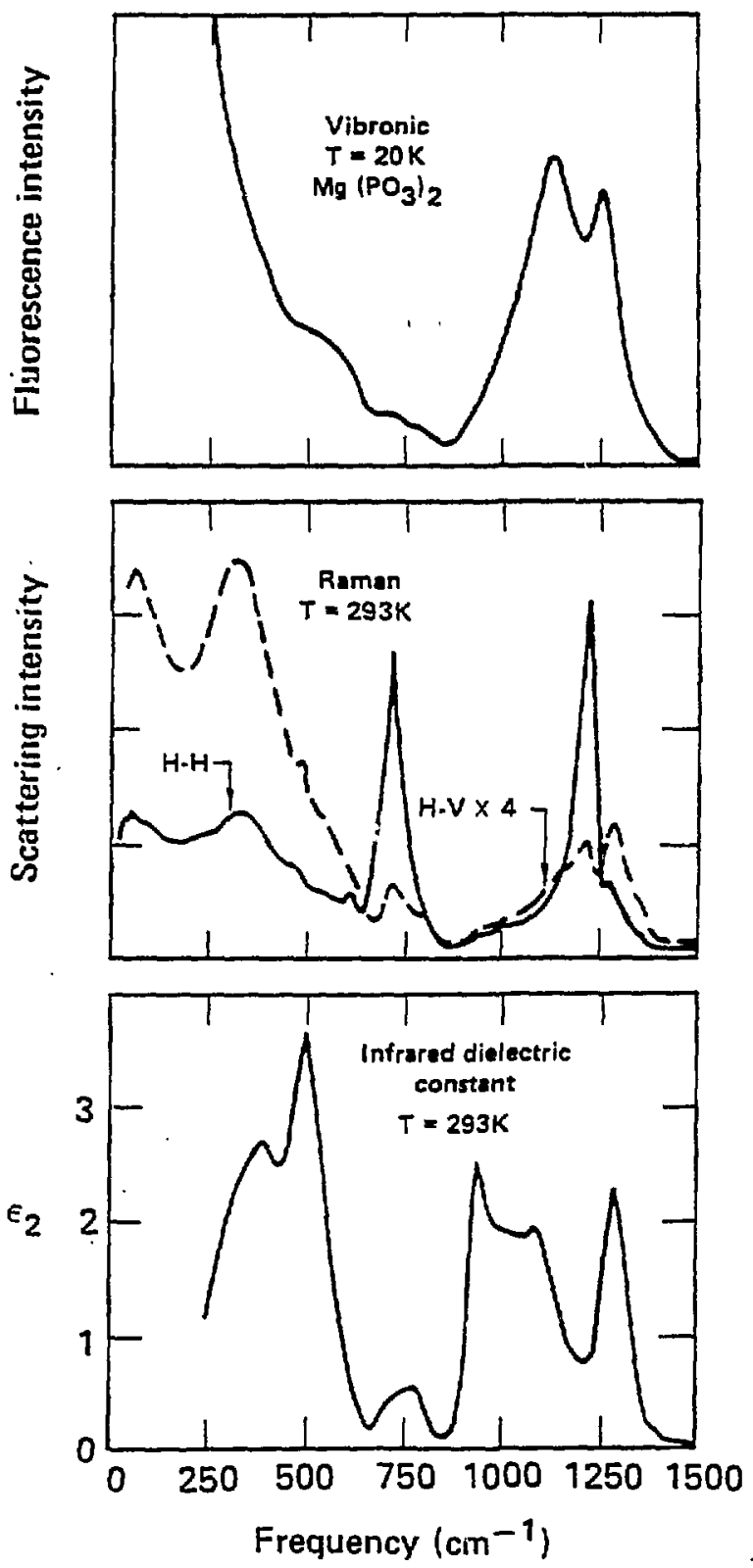

Figure 3: Comparison of the vibronic, $H H$ and $H V$ polarized Raman, and infrared (dielectric constant) spectra of $\mathrm{Mg}\left(\mathrm{PO}_{3}\right)_{2}$ : Gd glass. 
Fluorescence intensity

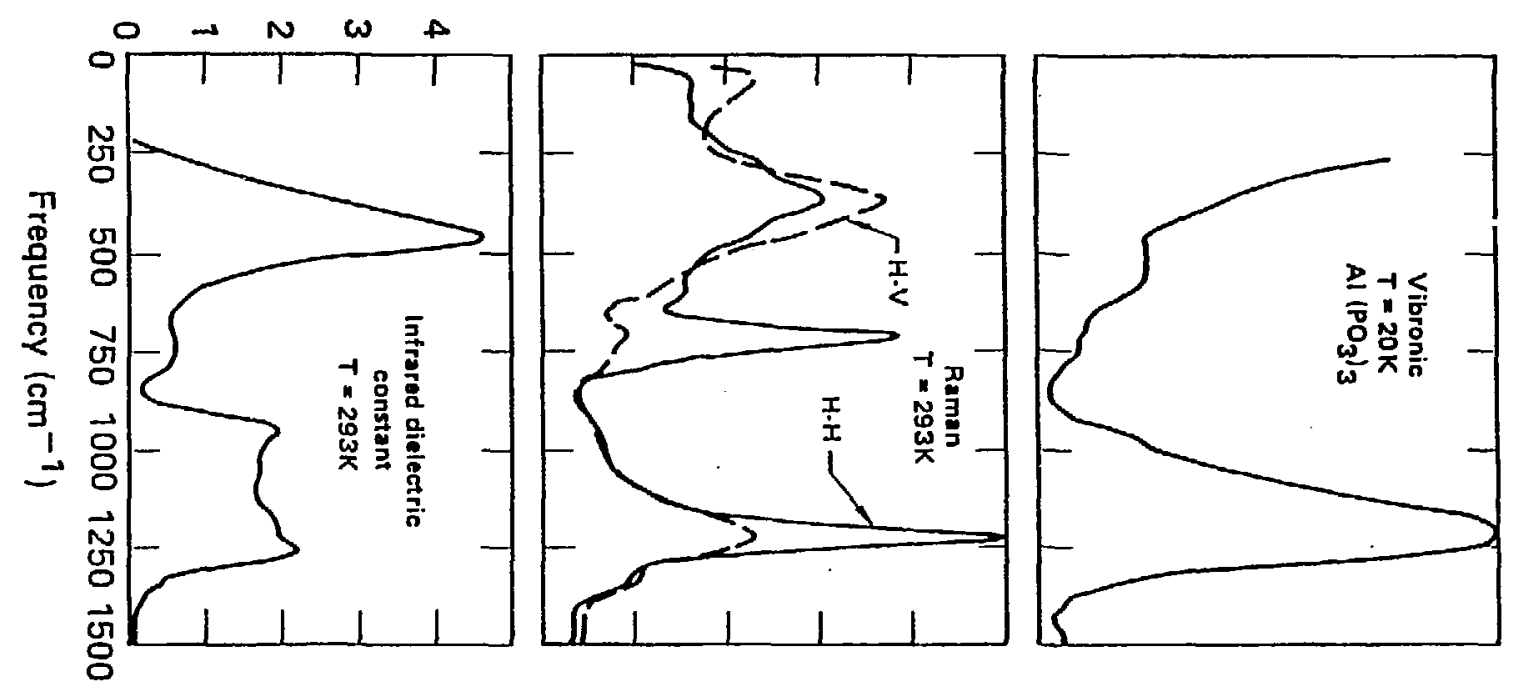



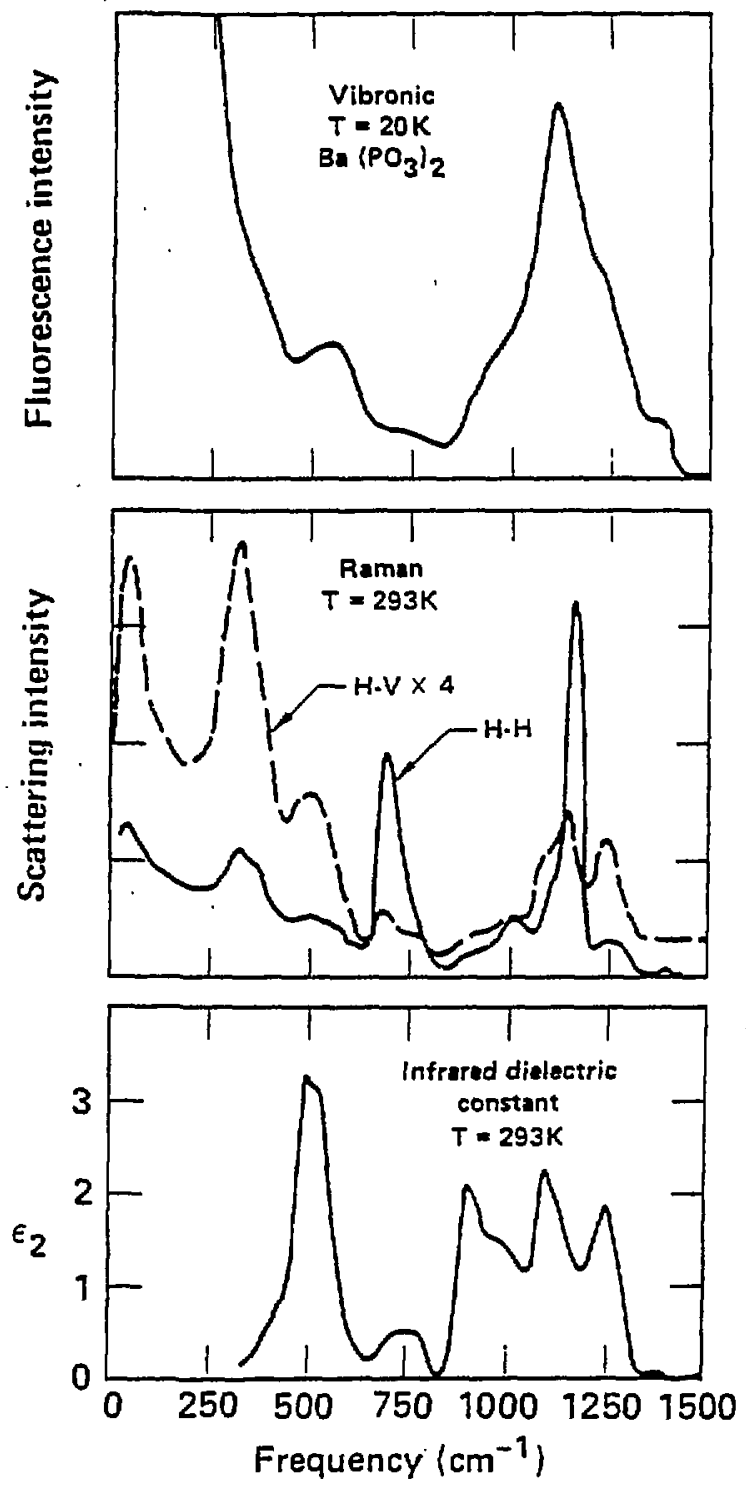

Figure 5: Comparison of the vibronic, HH and HV polarized Raman, and infrared (dielectric constant) spectra of $\mathrm{Ba}\left(\mathrm{PO}_{3}\right)_{2}: \mathrm{Gd} \mathrm{glass}$. 
The infrared reflectance of $\mathrm{La}\left(\mathrm{PO}_{3}\right)_{3}: \mathrm{Gd} \mathrm{glass}$ is shown in Figure 6 . The structure in this spectrum is typical of that observed for all of our glasses.

The infrared dielectric constant $\epsilon_{2}$ was obtained fron the IR reflectance spectrum and is also included in Figures 2-5. It was derived from a Kramers-Kronig transform performed using trapezoidal-rule integration on a POP-11/40 minicomputer. A discussion of the theory of Kramers-Kronig transforms, numerical methods, and the handling of unmeasured portions of the reflectance spectrum is included in Appendix $A$.

Frequencies of prominent features in vibronic, Raman, and infrared spectra are tabulated in Table I. Comparison of the high-frequency peaks from the RE-doped samples and from the undoped unpolarized data of Nelson \& Exarhos ${ }^{1}$ confirms that Raman scattering, which probes regions of spatial scale $>3-4 \mathrm{~nm}$, is not affected by the small addition of RE. ${ }^{2}$ B. Metaphosphate Glass Discussion

Before dealing with the vibronic spectra, we review the Raman and infrared spectra of the various metaphosphate glasses. Vibronic, Raman, and infrared spectra are strongly governed by selection rules. ${ }^{2}$ Because these rules are different for each spectroscopy, the relative intensities of paaks in the various spectra are not identical. The relationship of these spectra to the vibrational density of states depends on the selection rules and coupling strength. ${ }^{3,4}$ Since these depend on the nature of the vibrational modes, neither the vibronic, Raman, nor infrared spectrum provides a direct measure of the density of states. 


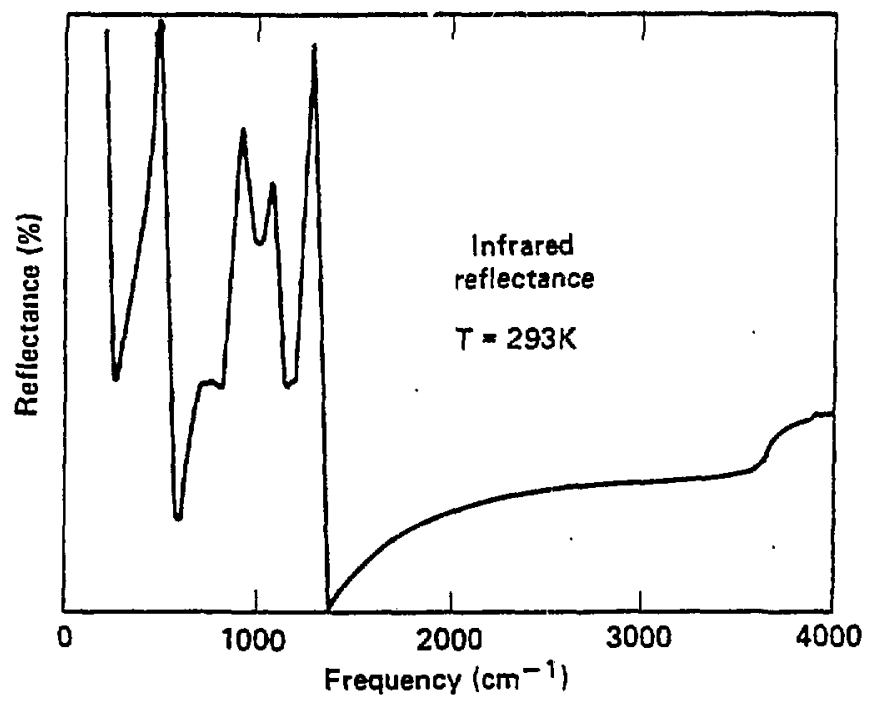

Figure 6: Infrared reflectance spectrum of $\mathrm{La}\left(\mathrm{PO}_{3}\right)_{3}: \mathrm{Gd}$ glass recorded at $15^{\circ}$ from normal. 
Table I. Comparison of peak frequencies observed in HV Raman ( $T=293 \mathrm{~K}$ ), infrared $(T=293 \mathrm{~K})$, and $\mathrm{Gd}^{3+}$ vibronic $(T=20 \mathrm{~K})$ spectra for various metaphosphate glasses. Typical uncertainties in determining the peak positions are indicated. All frequencies are in $\mathrm{cm}^{-1}$.

\begin{tabular}{lllll}
\hline $\mathrm{Glass}$ & $\mathrm{Mg}\left(\mathrm{PO}_{3}\right)_{2}$ & $\mathrm{AT}\left(\mathrm{PO}_{3}\right)_{3}$ & $\mathrm{Ba}\left(\mathrm{PO}_{3}\right)_{2}$ & $\mathrm{La}\left(\mathrm{PO}_{3}\right)_{3}$
\end{tabular}

HV Raman:

\begin{tabular}{|c|c|c|c|c|}
\hline RI & $1272 \pm 5$ & 1331 & 1249 & 1232 \\
\hline R2 & $1210 \pm 5$ & 1225 & 1152 & 1177 \\
\hline Pef. 1 & 1210 & 1220 & 1163 & 1181 \\
\hline R3 & $1130 \pm 15$ & - & 1095 & 1080 \\
\hline R4 & $803 \pm 10$ & 788 & 771 & 784 \\
\hline R5 & $707 \pm 5$ & 715 & 689 & 696 \\
\hline R6 & - & - & 495 & 500 \\
\hline R7 & 329 & 355 & 333 & 333 \\
\hline
\end{tabular}

Infrared:

$\begin{array}{lcccc}\text { I1 } & 1283 \pm 10 & 1259 & 1250 & 1259 \\ \text { I2 } & 1085 \pm 10 & - & 1100 & 1066 \\ \text { I3 } & 926 \pm 10 & 950 & 907 & 926 \\ \text { I4 } & 750 \pm 20 & 750 & 750 & 750 \\ \text { I5 } & 482 \pm 10 & 458 & 500 & 482 \\ \text { I6 } & 380 & 400 & - & - \\ \text { Ref. } 1 & 405 & 400 & 143 & 189 \\ \text { ic: } & & & & \\ \text { V1 } & 1245 \pm 15 & 1210 & 1225 & 1235 \\ \text { V2 } & - & - & - & 1170 \pm 15 \\ \text { V3 } & 1120 \pm 15 & - & 1110 & 1095 \\ \text { V4 } & 710 \pm 30 & 750 & 725 & 750 \\ \text { V5 } & 530 \pm 30 & 550 & 550 & 530\end{array}$


Raman spectra

Raman scattering spectra emphasize those vibrational modes with a displacement-dependent polarizability. Spectra of all of our metaphosphate glasses are qualitatively the same, reflecting the similarity of their basic structure regardless of the metal cation. The most prominent bands observed in the HH and HV polarizations occur in the vicinity of $50,30,700$, and $1200 \mathrm{~cm}^{-1}$; the exact location of peaks are given in Table I.

It is clear that the HH and HV spectra obey different selection rules. The two highest frequency bands exhibit structure and a strong polarization dep since, whereas the lower frequency bands do not. The HV spectra contain all features of the HH spectra (with different relative intensities) plus several additional peaks. These latter peaks are not resolved in previously reported unpolarized spectra, $1,5,6$ although there are hints of shoulders in these data.

The widths of the Raman lines are comparable for all glasses except for $\mathrm{Al}\left(\mathrm{PO}_{3}\right)_{3}$. The broader features in the latter glass are attributed to the ability of $A 1$ to be incorporated as both a glass network former and network modifier which introduces disorder and relaxes selection rules. The presence of $\mathrm{Al}$ in other oxide glasses also results in the broadening of spectral features. '

The high-frequency vibrational bands in metaphosphate glasses have been associated with motions within the phosphate chain with mode frequencies slightly perturbed by the modifier cations. Assignments of these (unpolarized) bands have been made by Bobovich. ${ }^{5}$ The band at 
$700 \mathrm{~cm}^{-1}$ (R5) was assigned to P-O-P stretching mades of the chain itself. The highest frequency band at $=120 \mathrm{~cm}^{-1}$ was assigned to motion of the two non-bridging oxygens per tetrahedron ( $P \ldots$ group) relative to the phosphorous-bridging oxygen polymeric chain. Specifically, the dominant high-frequency peak in the unpolarized Raman spectra (which is equivalent to the large HH peak at trequency labeled R2 in Table i) is assumed due to a $\mathrm{PO}_{2}$ symmetric stretch while a sate i ica at siightly higher frequency (RI) is assigned to a $\mathrm{PO}_{2}$ asymmetric stretch.

The frequencies of the two peaks $R 1$ and $R 2$ vary systematically with metal cation (see Table I). Rouse et al, ${ }^{6}$ have explained the shift of the highest frequency vibration band in different alkali metaphosphace glasses using a simple, four-particle vibrational model. In this model, mode frequencies depend on the metal-nonbridging oxygen force constant and the $\mathrm{PO}_{2}$ bond angle, both of which are governed primarily by metal cation size. They were successful in predicting a linear decrease of peak frequency with increasing cation size and different rates of change of the symmetric and asymmetric mode frequencies. Their model, however: does not contain enough degrees of freedom to explain all the high-frequency features of polarized Raman spectra. One example is the peak labeled $\mathrm{R} 3$, which is clearly resolvable in $\mathrm{La}\left(\mathrm{PO}_{3}\right)_{3}$ and appears as a definite shoulder in the other materials. This peak does not vary consistently with cation size. 


\section{Infrared spectra}

The $\epsilon_{2}$ spectra for ail of the metaphosphate glasses in Figures 2-5 are qualitatively similar, demonstrating again the commonality of their basic structures. Again, the $\mathrm{Al}\left(\mathrm{PO}_{3}\right)_{3}$ glass has broader features with no resolved structure in the highest-frequency band. Although the broad bands in the $\epsilon_{2}$ spectra at $=500$ and $750 \mathrm{~cm}^{-1}$ show general correspondence with bands in the Ramah spectra, high-frequency peaks such as 13 show no correspondence with strong peaks in either the Raman or vibronic spectra. The peak 16 arises from the vibration of the metal. cation in its cage of surrounding oxygens. The frequency of this cation motion has been studied in a series of metaphosphate glasses using far-infrared absorption datal and is included in Table I. A well-resolved peak corresponding to this mode is observed in the $\epsilon_{2}$ spectrum of $\mathrm{Mg}\left(\mathrm{PO}_{3}\right)_{2}$ glass and a shoulder is observed for the A) $\left(\mathrm{PO}_{3}\right)_{3} \mathrm{glass}$; for the other glasses the frequency of this mode is below our range of observation.

Inirared experiments probe those vibrational modes that create a macroscopic electric-dipole moment. The nature of the infrared-active vibrational modes in glass has been discussed by Galeener and Lucovsky. 8 These authors claim that peaks in the $\epsilon_{2}$ spectrum are assoc' iled with vibrations having transverse optical mode character whereas peaks in the energy loss function, - $\operatorname{Im}(1 / \epsilon)$, are associated with vibrations having longitudinal optical mrde character. They also report evidence of a longitudinal/transverse optic mode splitting in the polarized Raman spectra of $\mathrm{GeO}_{2}, \mathrm{SiO}_{2}$, and $\mathrm{BeF}_{2}$ analogous to that 
found in crystals. ${ }^{8,9}$ The definitive experiment to verify a $10 / T 0$ splitting in glass has not been performed.

It is not ax all clear whether in fact the above concepts of transverse and longitudinal character apply to glasses. In these materials, most modes are probably localized and high-frequency vibrations might more reasonably resemble a set of independent, nearly uncoupled oscillators. In particular, because of the inhomogeneous broadening of $\epsilon_{2}$ arising from the presence of vibrating entities with different frequencies, the function $-\operatorname{Im}(1 / \epsilon)$ does not represent the "longitudinal" vibrations of such entities. We have calculated the energy loss function from the IR reflectance sp:ctra. The loss function of $\mathrm{La}\left(\mathrm{PO}_{3}\right)_{3}$ :Gd glass is shown in Figure 7 and is typical of that obtained for all our giasses. Peaks in $-\operatorname{Im}(1 / \epsilon)$ do no correspond with strong peaks in the vibronic, Raman, or $\epsilon_{2}$ spectra for any of the four metaphosphate glasses.

It is well established that the short-range order in inorganic glasses and crystals with similar compositions (if they exist) is very similar. Also, for silicates, phosphates, and borates, similar vibrational selection rules are observed in glass and crystal. In both glass and crystal, vibrational modes which are strongly Raman active are also strongly polarized and nearly infrared inactive. Conversely, modes which are weakly Raman active tend to be depolarized and hence appear in the HV spectrum. The depolarized modes are often the ones that are strongly infrared active. This does not appear to be satisfied for metaphosphate glasses, because the $\epsilon_{2}$ spectra are quite different from the HV Raman spectra. 


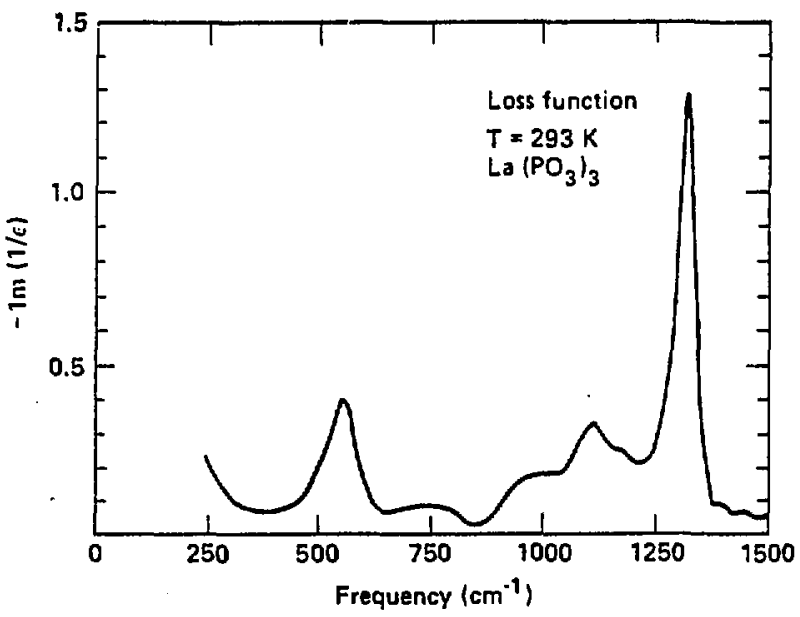

Figure 7: Energy loss function of. $\mathrm{La}\left(\mathrm{PO}_{3}\right)_{3}: \mathrm{Gd}$ glass. 


\section{Vibronic spectra}

Vibronic transitions involve an electronic transition accompanied by the creation/annihiliation of phonons. Thus, they probe those vibrations which create a displacement-dependent electric field at the RE site. This dynamic crystal field is a microscopic quantity in contrast to the macroscopic field involved in Raman and IR experiments. For simple crystals possessing tetrahedral symmetry, the macroscopic and microscopic electric fields are simply related. 10 For less symmetric crystals the relationship is complex. For impurity sites in glass the relationship is not clear.

The vibronic spectra of $\mathrm{Gd}^{3+}$ in metaphosphate glasses are similar to the Raman and IR spectra insofar as general bands are again observed in the vicinity of 500,750 , and $1200 \mathrm{~cm}^{-1}$. As noted earlier, lower frequency modes are obscurred by the tail of the zero-phonon line. Potentialiy intense modes involving motion of the RE dopant with respect to its neighboring oxygens are expected to occur at $\approx 200 \mathrm{~cm}^{-1}$ and hence are unresolved in our experiments. This estimate is based on far-infrared (FIR) absorption measurements ${ }^{1}$ of $\mathrm{Gd}\left(\mathrm{PO}_{3}\right)_{3}$ glass where the cation motion band is centered at $195 \mathrm{~cm}^{-1}$. Low-frequency vibrations associated with the metal cation occur at 143, 189, 405 and $400 \mathrm{~cm}^{-1}$ in the FIR. spectra of $\mathrm{Ba}, \mathrm{La}, \mathrm{Mg}$, and Al metaphosphate glasses, respectively. These bands would be partially or fully obscurred in our vibronic spectra.

The most striking result of a comparison of the different spectroscopies, apparent from Figures $2-5$, is the similarity of the 
high-frequency HV Raman and vibronic spectra. This is especially true for $\mathrm{La}\left(\mathrm{PO}_{3}\right)_{3} \mathrm{glass,}$ which is shown in greater detail in Figure 8 . In this material the RE(Gd) is most like the network modifying cation (La) the ionic charges are the same and the masses differ by only $12 \%$. The locations of the $R 1, R 2, R 3$ and $V 1, V 2, V 3$ peaks match within the spectral accuracy of our experiment; the relative intensities of the peaks are also nearly. the same. This correspondence implies that for metaphosphate glasses, vibronic selection rules are similar to those of HV Raman scattering.

In the $\mathrm{Mg}\left(\mathrm{PO}_{3}\right)_{2} \mathrm{glass}$, the RE is both a mass and a charge defect. Still, there is a twin-peaked high-frequency band in both the HV and vibronic spectra, but with a change in relative intensities and with the locations of the peaks in the vibronic spectrum lower by $\simeq 30 \mathrm{~cm}^{-1}$. This is illustrated in Figure 9. These differences are presumably the result of the perturbing RE. However, the broad band at $750 \mathrm{~cm}^{-1}$ and the shoulder at $\simeq 530 \mathrm{~cm}^{-1}$ coincide in frequency and relative intensity with those in the Raman spectrum.

Although the highest-frequency vibronic band of the $\mathrm{Ba}\left(\mathrm{PO}_{3}\right)_{2}$ glass is not twin-peaked, the locations of the maxima and shoulder of this band are the same as the two distinct high-frequency peaks in the HV Raman spectrum. The weaker bands at $=750$ and $550 \mathrm{~cm}^{-1}$ are generally the same in the two spectra.

The general features of the HV Raman and vibronic spectra of $\mathrm{Al}\left(\mathrm{PO}_{3}\right)_{3}$ glass are similar, however any correlation of peaks is obscurred by the broadening of spectral features mentioned previously. 
$-68-$

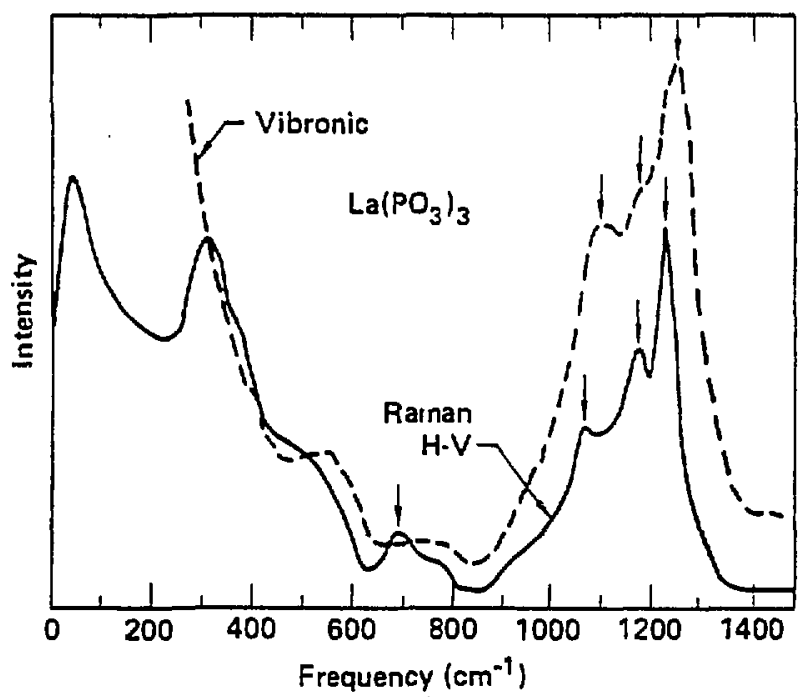

Figure 8: Detailed comparison of the vibronic and HV Ramen spectra of $\mathrm{La}\left(\mathrm{PO}_{3}\right)_{3}:$ Gd glass. 


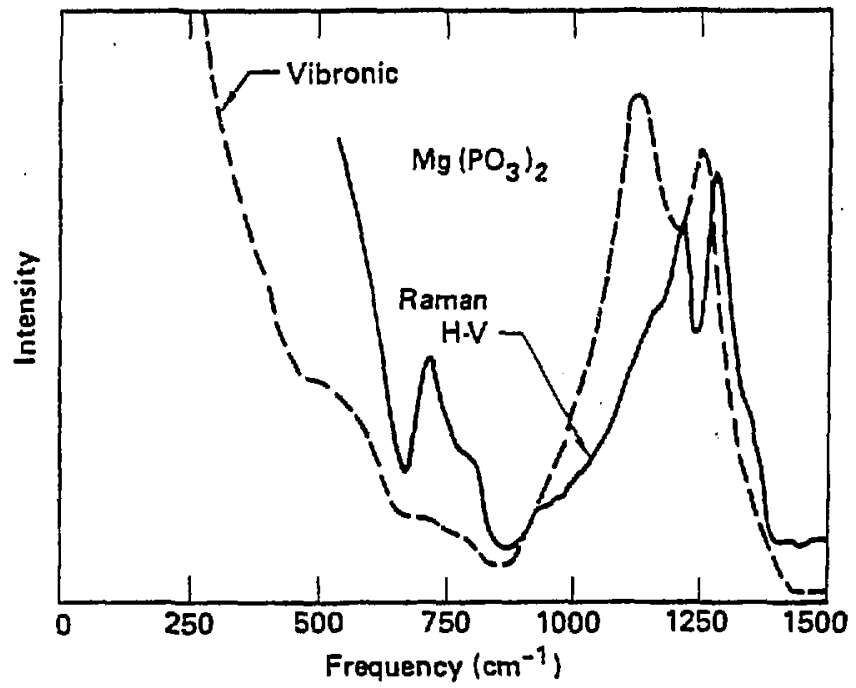

Figure 9: Detailed comparison of the vibronic and HV Raman spectra of $\mathrm{Mg}\left(\mathrm{PO}_{3}\right)_{2}$ : Gd glass. 
As seen in Figures 2-5, the vibronic spectra are very different in shape from either the HH Raman, or $\epsilon_{2}$ spectra. The vibronic spectrum is completely different from the $-\mathrm{Im}(1 / \epsilon)$ spectrum.

Vibronic transitions of rare-earth ions in solids are predominantly of electric-dipole nature. For $4 f-4 f$ transitions, they become allowed by admixing of states of opposite parity by the dynamic crystal field. Judd $^{11}$ has shown that the probability for one-phonon vibronic transitions of rare earths can be expressed in terms of a summation of products of phenomenological intensity parameters and matrix elements of tensor operators between states of $4 f^{n}$. The parameters involve terms of odd-order spherical harmonics in the expansion of the dynamic crystal field. The contributions from terms of order $N$ decrease with separation $R$ between the rare earth and neighboring ion as $1 / R^{N+1}$. Therefore, the contributions to the third and higher-order terms arise mainly from short-range interactions. The first-order term, however, decreases as $1 / R^{2}$ and hence is relatively long range. For transitions between states where all terms are allowed by electric-dipole selection rules, vibrations of both ligands and more distant neighbors can, in principle, contribute to phonon-assisted transitions.

As discussed at the end of the section of infrared spectra, the HV Raman spectrum consists of bands which might be expected to be infrared active. These modes have an associated induced dipole moment and would contribute strongly to the first-order terms in the dynamic crystal field. They would therefore be effective in inducing vibronic transitions of rare-earth ions. This could account for the similarity of the HV Raman and vibronic spectra. 
On the other hand, strongly polarized Raman modes in these materials are very weakly IR active, which is consistent with the differences between $\mathrm{HH}$ and vibronic spectra. However, the great difference between $\epsilon_{2}$ and vibronic spectra is not consistent with these ideas.

Rationalization of the above correlations requires a detailed knowledge of the motions of ions about the rare earth and the site-to-site variations throughout the glass. Simple molecular models of $\therefore$ local structure, while valuable for qualitative understanding of vibrations in bulk glass, probably will not suffice. An understanding of selection rules and ion-phonon coupling in glass on a microscopic scale is needed:

In summary, we find that fluorescence line-narrowed vibronic spectra of $\mathrm{Gd}^{3+}$ in glass provide an independent vibrational spectroscopy that differs from the more conventional spectroscopies (Raman, IR, neutron) in that it probes vibrations on a localized scale. We have found that in metaphosphate glasses:

1. The vibronic sideband of the $\mathrm{Gd}^{3+}{ }^{6} \mathrm{P}_{7 / 2} \rightarrow{ }^{8} \mathrm{~S}_{7 / 2}$

fluorescence are weak but provide a clear, unambiguous system for studies of vibrations in solids.

2. In metaphosphate $\mathrm{glasses,} \mathrm{when} \mathrm{the} \mathrm{Gd}^{3+}$ probe ion is similar to the glass modifier cation in mass and charge, the vioronic spectrum reflects selection rules and relative intensities comparable to those for iN Rainain scattering. 
3. When the $\mathrm{Gd}^{3+}$ probe ion differs from modifier cation in charge or mass, gross features of the vibronic and HV Raman spectra are similar, however vibrational frequencies associated with the network former $\left(\mathrm{PO}_{4}\right)$ may be perturbed.

4. Vibronic spectra of $\mathrm{Gd}^{3+}$ in metaphosphate glasses differ greatly from HH Raman and $\epsilon_{2}$ spectra. 


\section{BeF 2 Glass Results}

Room temperature fluorescence of the ${ }^{6} \mathrm{P}_{7 / 2} \rightarrow{ }^{8} \mathrm{~S}_{7 / 2}$ transition of $\mathrm{Gd}^{3+}$ in beryllium fluoride is shown in Figure 10. The fluorescence was laser-excited at $310.8 \mathrm{~nm}$; the observed sharp peak resonant with the laser superimposed on the broader fluorescence band is evidence of some site selectivity. Since the actual laser excitation occurred near the center of the band, it can couple to, many site types through accidental coincidences. The lack of total line narrowing is a result of accidental coincidences and emission from all stark levels of the ${ }^{6} p_{7 / 2}$ level due to their large Boltzmann population at room temperature. The wider portion of the spectrum is very much like fluorescence excited with a broad band light source. Weak vibronic transitions are evident by the asymmetric long-wavelength tail of the band and by structure near $318.5 \mathrm{~nm}$.

Comparing the room temperature spectrum of the $\mathrm{Gd}^{3+}$ transition in a metaphosphate glass (Figure I) with that in the BeF 2 glass (Figure 10) shows that the peak of the broad zero-phonon fluorescence occurs at a slightly shorter wavelength in $\mathrm{BeF}_{2}$. The width of the band, which results from the combined effects of Stark splitting and the inhomogeneous broadening of individual components, is $\simeq 150 \mathrm{~cm}^{-1}$ in $\mathrm{BeF}_{2}$, as compared to $\simeq 300 \mathrm{~cm}^{-1}$ in $\mathrm{Ba}\left(\mathrm{PO}_{3}\right)_{2}$. Also, the first e-folding decay time of the ${ }^{6} P_{7 / 2}$ level in $B_{e} F_{2}$ is $10.0 \mathrm{~ms}$, while it is only $4.2 \mathrm{~ms}$ in the metaphosphate glass.

Low-temperature $(20 \mathrm{~K})$ spectra of the vibronic sidebands of the ${ }^{6} \mathrm{P}_{7 / 2} \rightarrow{ }^{8} \mathrm{~S}_{7 / 2}$ transition were recorded with 14 excitation wavelengths $(310.40$ to $311.85 \mathrm{~nm})$ across the absorption/fluorescence band. (The extreme short-wavelength wing of the band was not probed). 


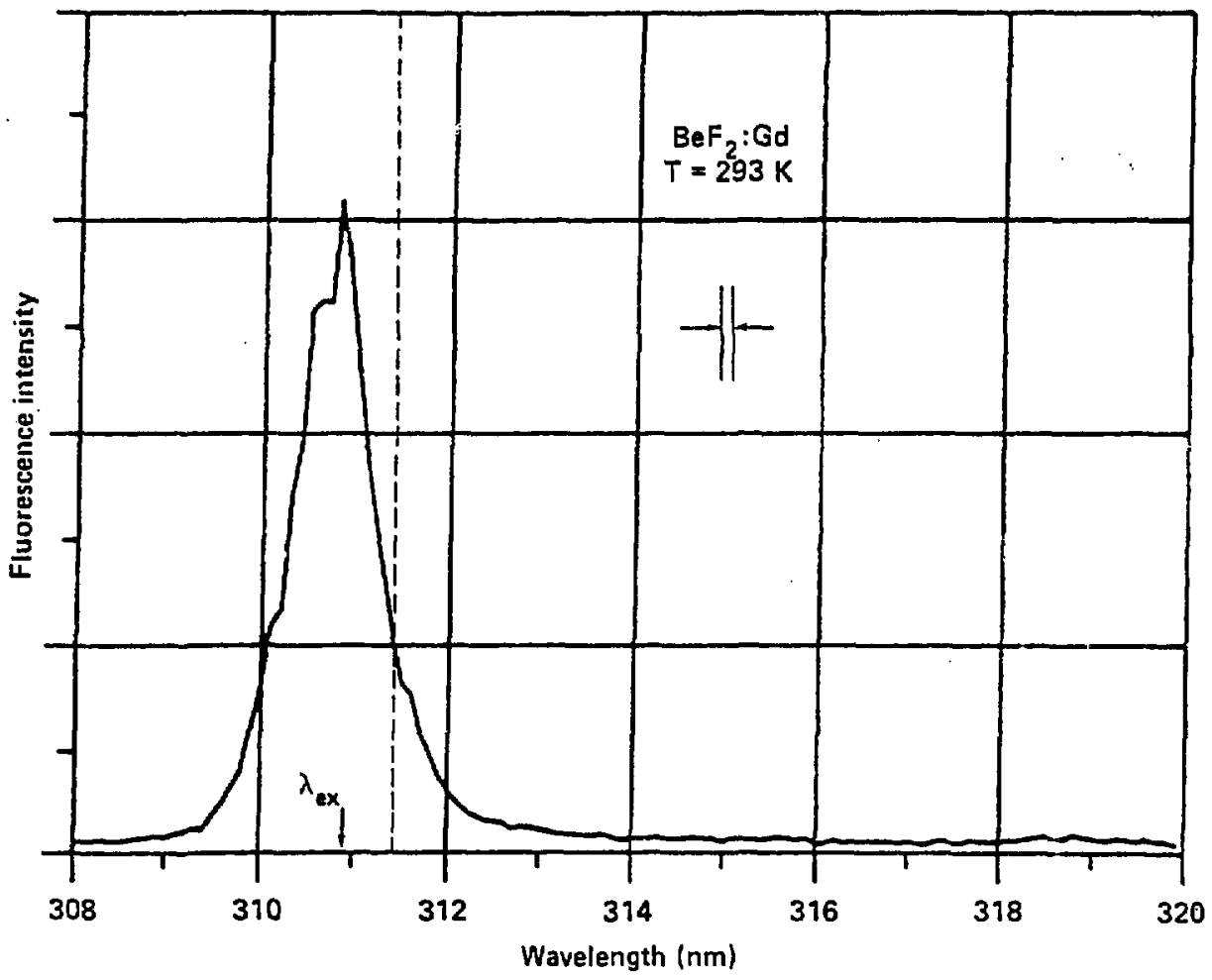

Figure 10: Room temperature laser-excited $6 p_{7 / 2} \rightarrow 8_{57 / 2}$ fluorescence of $\mathrm{Gd}^{3+}$ in $\mathrm{BeF}_{2} \mathrm{glass}$. Excitation wavelength: $310.8 \mathrm{~nm}$. 
The sharp line-narrowed zero-phonon line of low-temperature spectra was accompanied by a broader fluorescence background on the long-wavelength side when the exciting wavelength was shorter th? $311.4 \mathrm{~nm}$, (indicated by the dashed line in Figure 10). With laser wavelengths $\geq 311.4 \mathrm{~nm}$, only a sharp line was observed.

Vibronic spectra, plotted with abscissa representing the frequency shift of structure from the zero-phonon line, were identical wi+ain the spectral accuracy and resolution of the experiment for all laser wavelengths $\leq 311.4 \mathrm{~nm}$. An example of the spectrum $\left(\lambda_{\text {ex }}=311.4 \mathrm{~nm}\right)$ is shown in Figure 11 along with the reduced neutron spectrum of $\mathrm{BeF}_{2}$ glass from Reference 12. Figure 22 compares the same vibrenic spectrum with the reduced Raman and $\epsilon_{2}$ spectra. ${ }^{12}$ The neutron ar 4 Raman spectra are "reduced" in that the room-temperature phonon population factor and frequency dependencies characteristic of the particular scattering process are removed. Galeener and $\operatorname{Sen}^{13}$ assert that these reduced spectra, as well as a frequency-weighted dielectric constant $\left(\epsilon_{2}\right)$, are most representative of the vibrational density of states of the material. The HV polarized Raman spectrum of $\mathrm{BeF}_{2}$ is extremely weak and structure is very much like the HH spectrum.

For exciting wavelengths shorter than $311.4 \mathrm{~nm}$, the vibronic spectra have the same general spectral features as in Figure 12. However, peaks in the spectra no longer "track" the laser frequency but occur at the same absolute frequency, irrespective of the exciting laser frequency. Also, peaks in the vibronic spectrum are wider. For example, the large sideband at $\simeq 800 \mathrm{~cm}^{-1}$ in Figure 11 has a full width at half-maximum of $98 \pm 5 \mathrm{~cm}^{-1}$ while the same band has a width of $128 \pm 5 \mathrm{~cm}^{-1}$ in a spectrum excited at $311.1 \mathrm{~nm}$. 

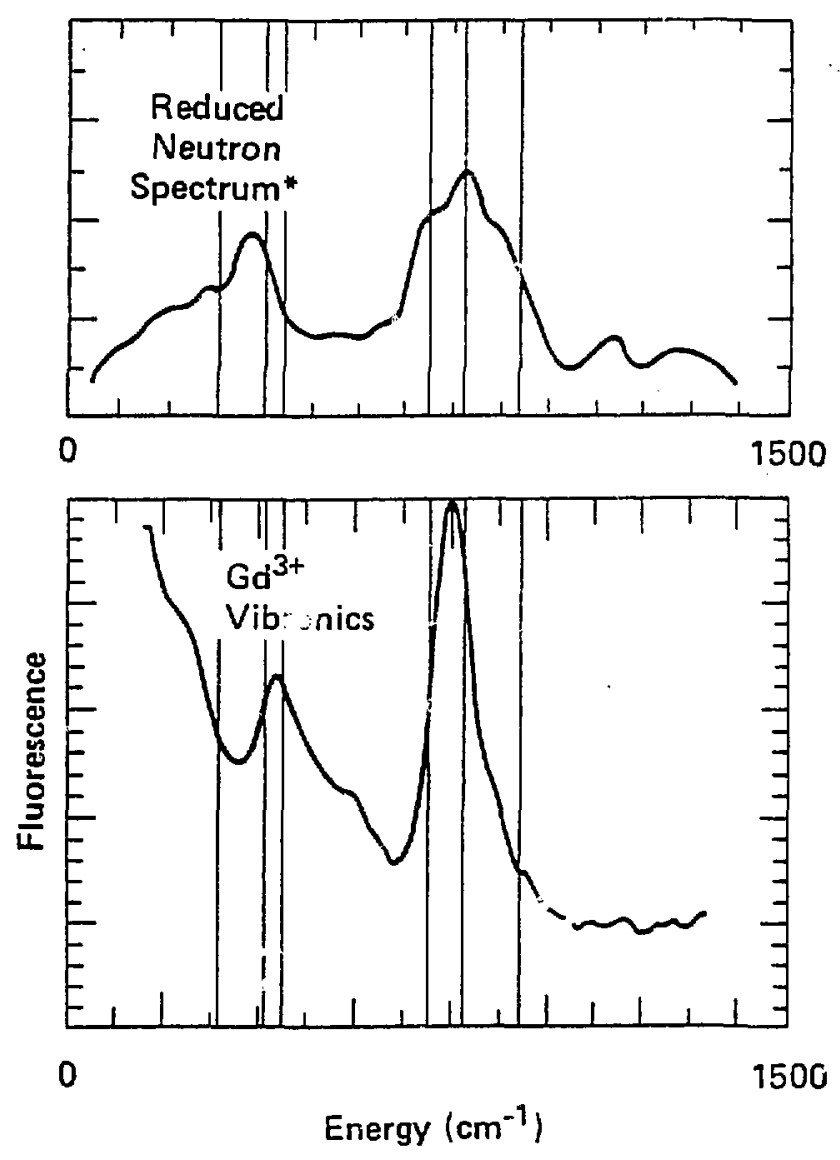

*Galeener, Leadbetter, Stringfellow (to be puljlished)

Figure 11: Inelastic neutron scattering and vibronic spectra of $\mathrm{BeF}_{2}$ glas". Excitation wavelength for the vibronic spectrum: $311.4 \mathrm{~nm}$. 

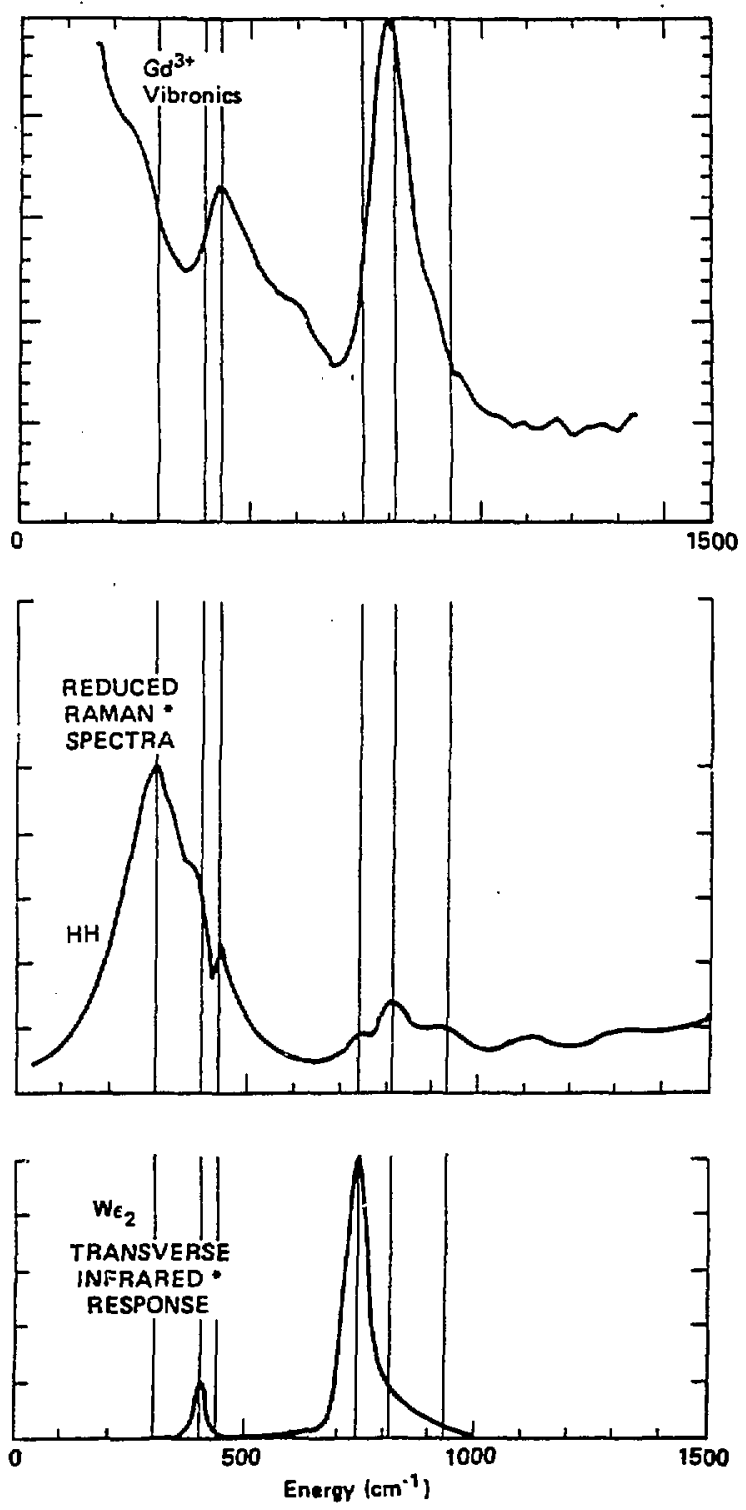

- Gatemer, Leadbetter, Stringfellow (to be published)

Figure 12: Vibronic, Raman, and infrared spectra of Bef 2 glass. 
D. BeF 2 Discussion

The narrower width of the Stark splitting and inhomogeneous broadening of the ${ }^{6} \mathrm{P}_{7 / 2}$ level of $\mathrm{Gd}$ in $\mathrm{BeF}_{2}$, as compared to an oxide glass, is typical of fluorine-based hosts ${ }^{14}$ and refiects a smaller interaction between the RE and its surrounding anions. The increased decay time constant for the fluaride glass is also indicative of decreased interaction.

The lack of complete line narrowing and the fallure of vibronic spectra to be shifted from the frequency of the laser when excitation is mid-band is an obvious manifestation of accidental coincidences. Some ions are excited to higher-energy Stark states of the level, relaxing to the lowest energy state before fluorescing. These higher-energy states have larger homogeneous linewidths and manly subsets of sites are excited. Vibronic spectra become a superposition of sidebands shifted a certain amount, $\Delta \omega$, from zero-phonon lines with differeni absolute frequency; peaks become fixed at absolute frequencies corresponding to vibrationai frequency shifts from an average zero-phonon line resulting from transitions from the lowest Stark state of almost all ions. The observed increased width of vibronic structure is consistent with the idea of accidental coincidences.

When spectra are obtained with excitation in the low-energy wing of the band, absorption is only possible via transitions to the lowest energy Stark state. Fluorescence occurs from the same state and the vibronic sidebands are shifted from zero-phonon lines that have a single frequency. 
Comparison of vibronic and the other spectroscopies of Figures 11 and 12 shows two prominent bands in each spectrum centered around 400 and 800 . $\mathrm{cm}^{-1}$. The relative intensities, widths and precise energies of peaks vary between the spectra, however. Selection rules governing vibronic transitions in $\mathrm{BeF}_{2}$ are different from those of the other vibrational spectroscopics including HV Raman scattering. It is generally believed ${ }^{15}$ that the reduced inelastic neutron scattering spectrum is the most faithful representation of the vibrational density of states (DOS) of a glass. Recent calculations by Brawer, 16 based on molecular-dynamics-simulated glass structure, show a significant variation between the DOS and neutron spectrum of $\mathrm{BeF}_{2}$.

Vibronic spectra of $\mathrm{BeF}_{2}$ and the metaphosphate glasses both show dominant high-energy peaks with the $B_{2} F_{2}$ peak occurring at $800 \mathrm{~cm}^{-1}$ and the peak in $\mathrm{Al}\left(\mathrm{PO}_{3}\right)_{3}$, for example, occurring at $1210 \mathrm{~cm}^{-1}$. This is consistent with the qualitative association of this feature with the stretching motions of the glass forming tetrahedra. The interatomic force constant for the P-O bond in metaphosphate glass, derived from Raman data, ${ }^{6}$ is about 3.7 times that for the Be-F bond. ${ }^{17} \mathrm{~A}$ simple-minded application of $\omega=(\mathrm{k} / \mathrm{m})^{\frac{1}{2}}$ for a harmonic spring implies a tetrahedral frequency in the phosphate that is 1.9 times higher than that in Bef $_{2}$; we see an actual increase by a factor of 1.5.

The major result of the $B_{2} F_{2}$ vibronic experiments is that spectra from the different subsets of ions selected by different excitation energies are identical within our spectral resolution, even when line riarrowing is complete. Two explanations for this observation are related 
to 1) the long-range nature of the ion-phonon interaction, and 2) accidental coincidences of resonant transitions.

The possibility of interaction terms in the dynamic crystal field with a $1 / R^{2}$ dependence was discussed in connection with the metaphosphate glass results (Section $B$ ). If the RE interacts with atoms out to its second or third coordination sphere ( $B e$ and next-nearest $F$, respectively), it will be influenced by vibrations in a significant volume of the glass. The total effect on the RE will be from vibrations of many local structures that average variations, resulting in a similar dynamic crystal field at each RE site. Differences due to an ion's immediate environment, which determine its electron state energies, would not be very significant and spectra from site-to-site should be similar. The long-range nature of the interaction may be especially important in BeF $_{2}$ compared to oxide glasses as a result of a "reduced-mass" effect: the motion of the light beryllium ion in the second coordination sphere should account for most of the total motion of Gd-F-Be vibrations. This explanation was used by Brecher et al.$^{14}$ to justify a decreased site-to-site dependency in the phonon-mediated nonradiative decay rate in Nd:Borate glass (Boron AtWt=11) as compared to $\mathrm{Nd}$ :Silicate glass (Silicon Atwt $=32$ ). The influence of the anion motion should be even more pronounced with the light, ionic, and doubly-charged $\mathrm{Be}$.

Another plausible explanation for the identical spectra, irrespective of the range of interaction, is that the subset of ions excited by the laser has a wide variety of structures. This would result in spectra that are superpositions of sidebands from sites with different 
vibrational modes, even though the sites all have electronic energy levels separated by the same frequency. Intuitively, ions with the same energy levels should have similar local structure. However, calculations of the energy eigenvalues of $\mathrm{Eu}^{3+}$ in different $\mathrm{BeF}_{2}$ environments, determined by molecular-dynamic simulations, ${ }^{18}$ have shown this need not be true. Hence, one can have line narrowing, in which a subset of sites resonant with the laser are selected, without having complete site-selectivity.

It was found in these simulations that sites of a subset, although of different structure, still had many things in common. For example, the total extent of Stark splitting of states within a subset was almost the same i.e., all energy levels were approximately coincident. It may be that sites of a subset exhibit similar coupling strengths to lattice modes; this would be manifested experimentally by a constant ratio of vibronic peak intensity to zero-phonon line intensity for a given subset. This possibility warrants further investigation.

The two explanations presented are not mutually exclusive. The identical spectra may result from a combination of the effects or from other unknown effects. The conclusion that must be drawn from the result is that vibronic spectra of $\mathrm{Gd}^{3+}$ in $\mathrm{BeF}_{2}$ do not exhibit differences with excitation wavelength--differences known to exist in glass from measurements of frequencies, linewidths, and transition probabilities. 19 This frustrates its use as a tool to verify local structures in $\mathrm{BeF}_{2}$ by their characteristic frequencies but does not negate its value as a probe of vibrations in glass that is governed by selection rules which are different from the more conventional spectroscopies. 


\section{References}

1. B. N. Nelson and G. J. Exarhos, J. Chem Phys 71, 2739 (1979).

2. S. A. E awer, Phys. Rev. B 113173 (1975).

3. R. S. Shuker and R. W. Gammon, Phys. Rev. Lett. 25, 222 (1970).

4. F. L. Galeener and P. N. Sen, Phys. Rev. B 17, 1928 (1978).

5. Ya. S. Bobovich, Opt. Spectrosc. (USSR) 13,274 (1962).

6. G. B. Rouse, P. J. Miller, W. M. Risen, J. Non-Cryst. Solids 28, 193 (1978).

7. S. A. Brawer and W. B. White J. Non-Cryst. Solids 23, 261 (1977).

8. F. L. Galeener and G. Lucovsky, Phys, Rev. Lett. 37, 1474 (1976).

9. F. L. Galeener, G. Lucovsky and R. H. Geils, Solid State Comm. 25, 405 (1978).

10. M. Born and K. Huang, Dynamical Theory of Crystal Lattices (Oxford, University Press, London, 1954), p. 101.

11. B. R. Judd, Phys. Rev. 127, 750 (1962).

12. F. L. Galeener, A. J. Leadbetter, and M. W. Stringfellow (to be published).

13. F. L. Galeener and P. N. Sen, Phys. Rev. B 171928 (1978).

14. C. Brecher, L. A. Riseberg, and M. J. Weber, Phys. Rev. B 185799 (1978).

15. D. Weaire and P. C. Taylor, in Dynamical Properties of Solids, eds. G. K. Hortion and A. A. Maradudin, (North-Holland, 1980) V. 4, p. 26.

16. S. A. Brawer, (private communication).

17. G. E. WaTrafen and R. H. Stolen, Solid State Comm. 21417 (1977).

18. S. A. Brawer and M. J. Weber, J. Non-Cryst. Solids 38/39 9 (1980).

19. M. J. Weber and S. A. Brawer, presented at 1981 Beijing International Symposium an Glass, J. Non-Cryst. Solids (to be published). 
Chapter 6: CRYSTAL RESULTS AND DISCUSSION

A. Fluorite Lattice ${ }^{1}$

Pure $\mathrm{CaF}_{2}$ normally crystallizes in the fluorite structure, which may be envisioned as a simple cubic lattice of $\mathrm{F}^{-}$ions with $\mathrm{Ca}^{2+}$ ions placed in the centers of alternate cubes. Triply-charged RE dopants enter the lattice substitutionaliy at $\mathrm{Ca}^{2+}$ sites and require some mechanism for charge compensation to keep the lattice electrically neutral. Figure 1 depicts two established compensation mechanisms involving interstitial $\mathrm{F}^{-}$ions. A nearest-neighbor $(n . n$.$) interstitial$ $\mathrm{F}^{-}$creates a site of tetragonal symmetry $\left(\mathrm{C}_{4 V}\right)$ for the RE and a next-nearest-neighbor (n.n.n.) $F^{-}$ion crates a trigonal $\left(C_{3 v}\right)$ site. Compensation by an interstitial $\mathrm{F}^{-}$at a distance of more than a few lattice constants from the RE creates a site of essentially cubic symmetry $\left(0_{h}\right)$. Compensation mechanisms involving intentional and unintentional impurity ions (e.g. $\mathrm{O}^{2-}, \mathrm{K}^{+}, \mathrm{Ag}^{+}$) have been investigated. ${ }^{2,3}$ For example, an $0^{2-}-R^{3+}$ pair may form with the $\mathrm{O}^{2-}$ iori occupying one of the eight $\mathrm{F}^{-}$lattice sites nearest to the RE ion; this results in a trigonal $\left(C_{3 v}\right)$ RE environment.

The lattice constant shown in Figure 1 is that of the pure lattice; the presence of the RE and charge compensating ion will modify the interionic separations. B. $\mathrm{Gd}^{3+}$ Energy Levels

The removal of degeneracy of the free-ion J-levels of $\mathrm{Gd}^{3+}$ by a crystal field with the point group symmetry of the various chargecompensating mechanisms may be determined by finding the irreducible 


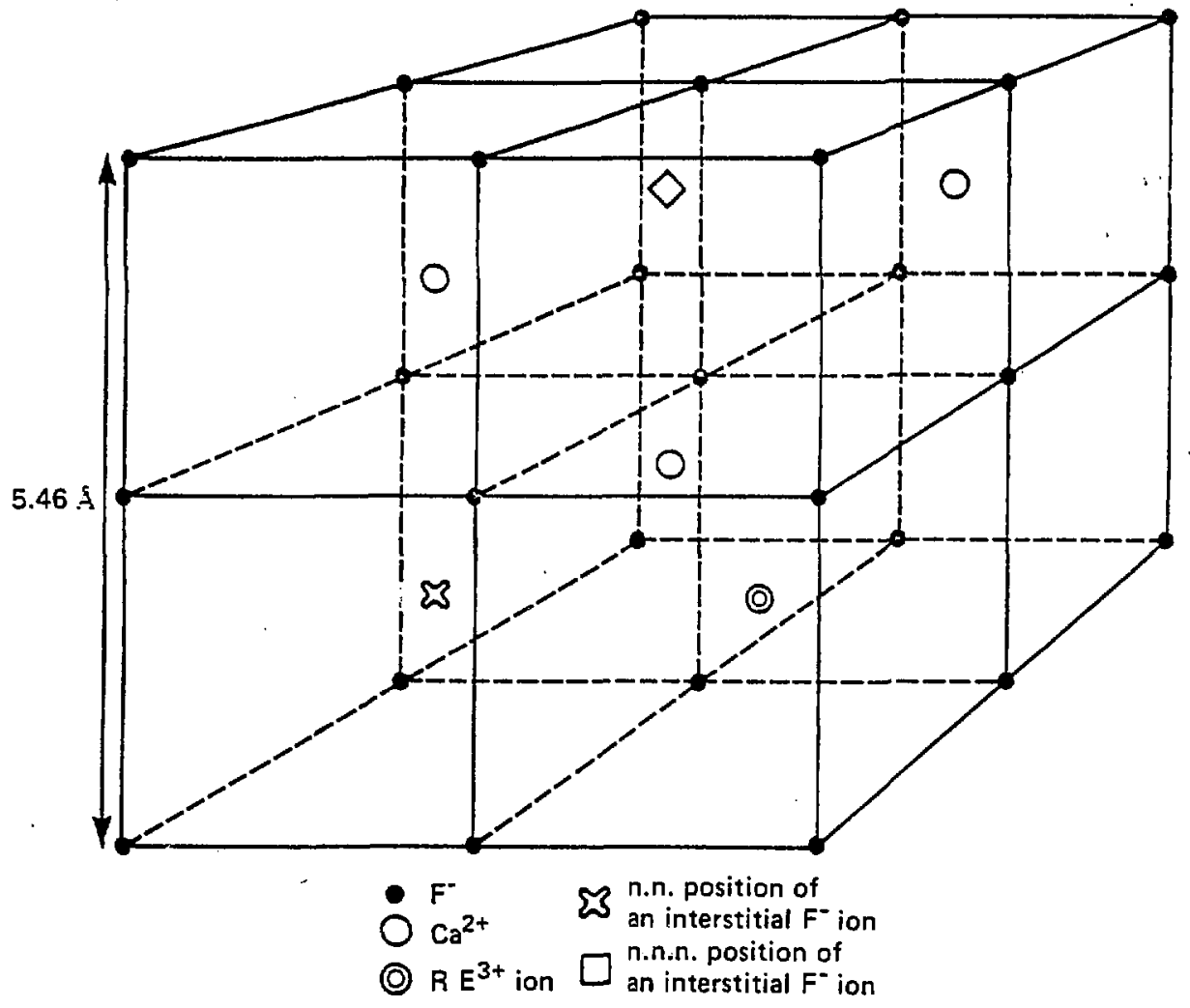

Figure 1: Schenatic representation of a single $\mathrm{RE}^{3+}$ in $\mathrm{CaF}_{2}$ showing two possible locations of charge-compensating interstitial $F^{-}$ions. 
representations for which the free-ion eigenfunctions form bases. 4 . The . degeneracy of both the ${ }^{6} P_{7 / 2}$ and ${ }^{8} s_{7 / 2}$ levels is completely. removed (except for the two-fold Kramers' degeneracy) in $C_{3 v}$ and $C_{4 v}$ sites, yielding four distinct Stark states for each level. For cubic sites, three distinct sublevels are predicted and observed; the energy ordering of the irreducible representations of the ${ }^{6} P_{7 / 2}$ states has been determined by the optical zeeman effect. ${ }^{5}$ The splitting of the ${ }^{8} \mathrm{~S}_{7 / 2}$ ground state, however, is extremely small $10.149 \mathrm{~cm}^{-1}$ in cubic sites ${ }^{6}$ ) and it may be treated as a single level. This is because the orbital portion of the many-electron wavefunctions of $S$ states are identical and the crystal-field interaction (in first order) depends only on the spatial distribution of the states.

Fluorescence from zero-phonon ${ }^{6} \mathrm{P}_{7 / 2} \rightarrow^{8} 5_{7 / 2}$ transitions of $\mathrm{Gd}^{3+}$ in $\mathrm{CaF}_{2}$, excited by laser light tuned to the middle of a band of absorption lines, is shown in Figure 2, revealing a superposition of lines from several sites. Careful tuning of the laser allows selection of a single site, as shown in Figure 3. Selection of sites whose lowest-energy Stark state is in the middle of the band was often frustrated by accidental coincidences with higher-energy Stark states of other sites that have large homugeneous linewidths. Site-selectivity could be improved by lowering the sample temperature, but this would prevent observation of flunrescence from all Stark states thich is necessary for a complete identification of the site.

Three distinct sites were isolated and their frequencies and lifetimes are listed in Table I. The first and third sites correspond to 


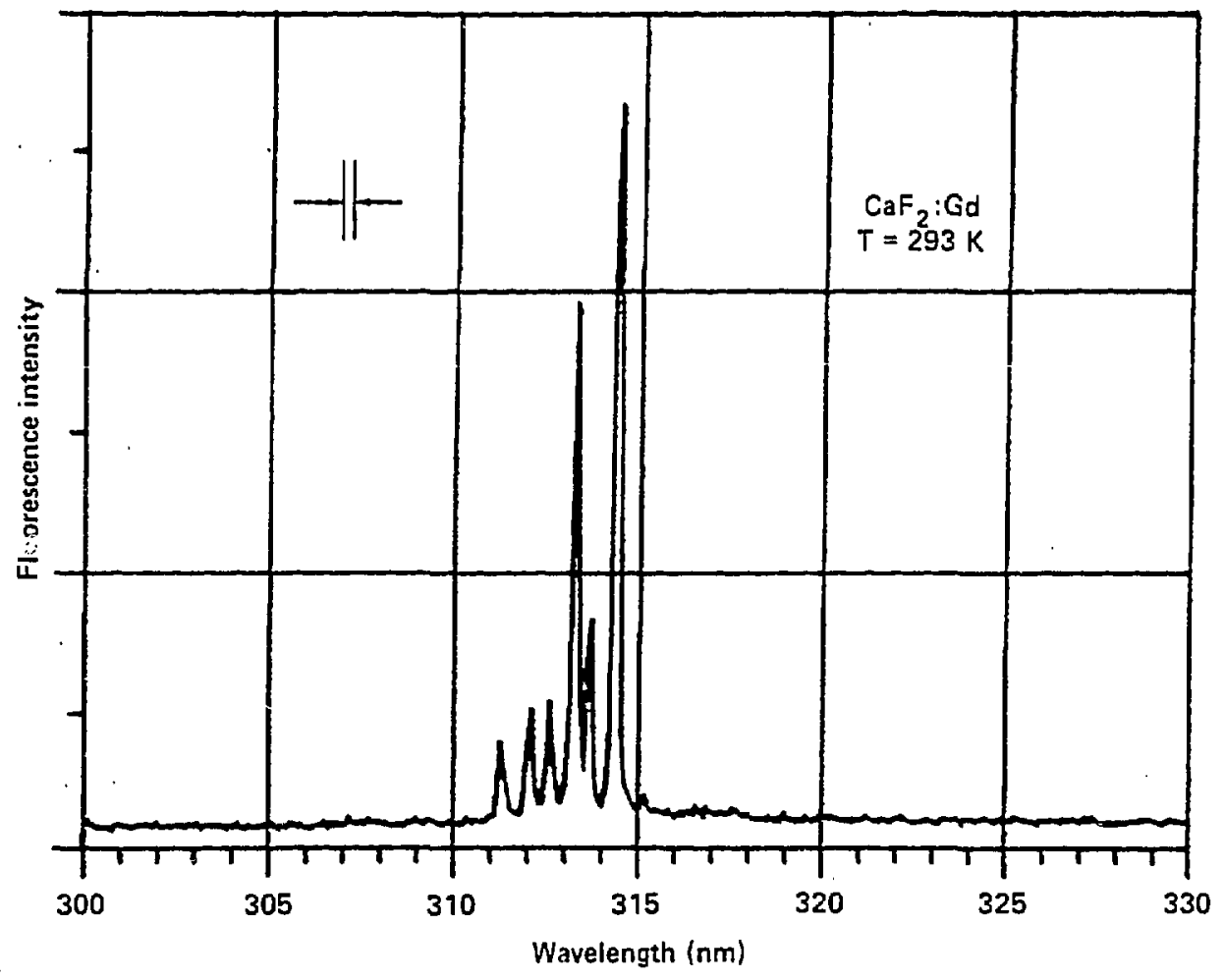

Figure 2: $\quad 6 \mathrm{P}_{7 / 2} \rightarrow{ }^{8} \mathrm{~S}_{7 / 2}$ fluorescence of $\mathrm{Gd}^{3+}$ in $\mathrm{CaF}_{2}$ with laser excitation randomly set at mid-band. 


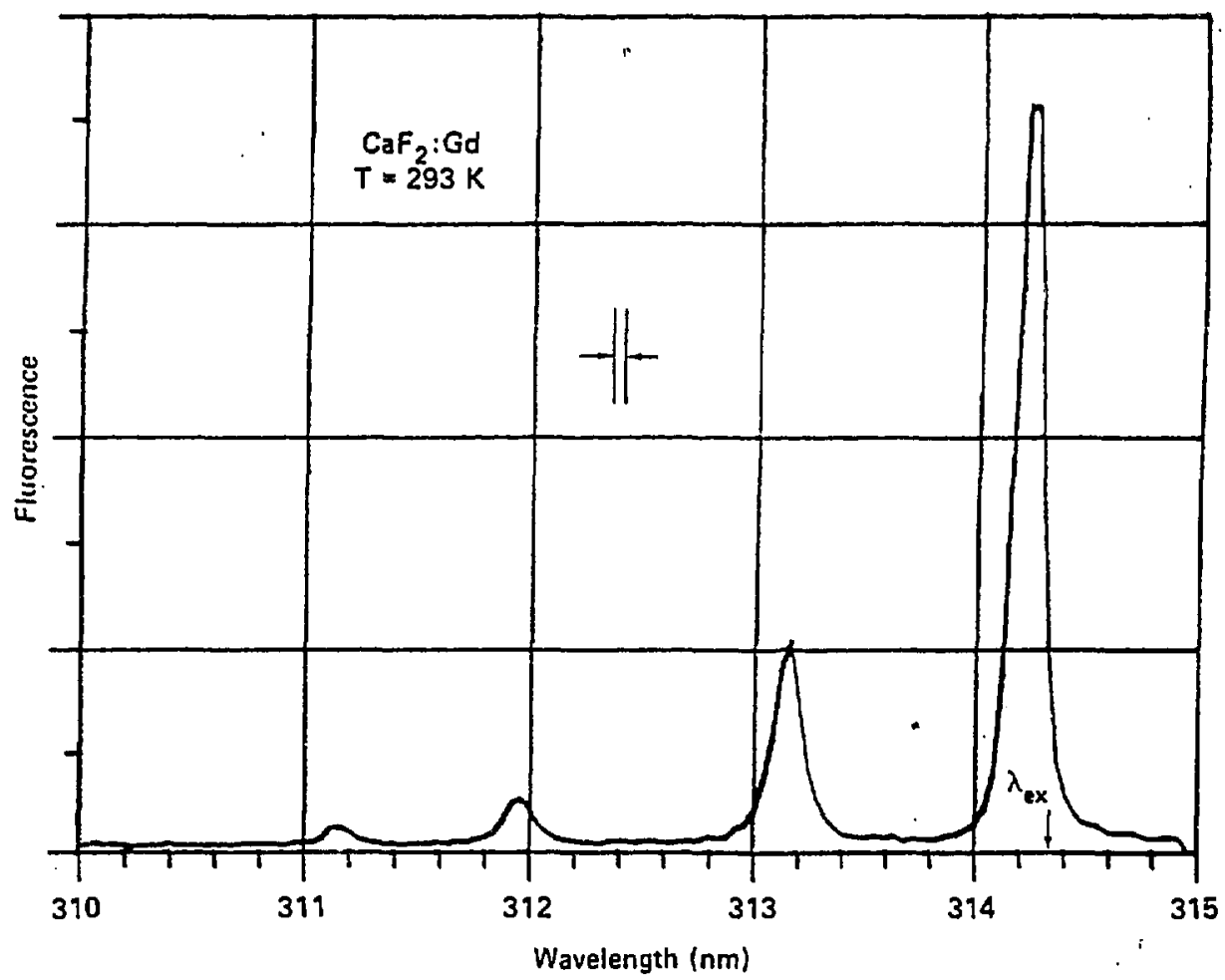

Figure 3: $\quad 6 \mathrm{P}_{7 / 2} \rightarrow{ }^{8} \mathrm{~S}_{7 / 2}$ fluorescence of $\mathrm{Gd}^{3+}$ in a single site of $\mathrm{CaF}_{2}$. 
$-88-$

Table I. Frequencies and decay time constants of the $6 \mathrm{p}_{7 / 2} \rightarrow{ }^{8} \mathrm{~S}_{7 / 2}$, transition of $\mathrm{Gd}^{3+}$ in $\mathrm{CaF}_{2}$ for three sites. All frequencies in $\mathrm{cm}^{2}$.

Site 1

Ref. 7

$\underline{\text { Site } 2}$

$$
31855 \quad 31893 \quad 3196 \% \quad 32056
$$

$\underline{\text { Site } 3}$

$\begin{array}{llllll} & 32046 & 32061 & 32102 & -- & 10.2 \\ \text { Ref. 7 } & 32036 & 32051 & 32092 & -- & --\end{array}$


ones identified by Makovsky, ${ }^{7}$ although the second daes not. (There is a systematic difference of approximately $10 \mathrm{~cm}^{-1}$ in the frequencies of lines between the two sets of data. Calibration of our spectrometer was accomplished using the sharp UV lines from a $\mathrm{Hg}$ light source).

Site 3 (Makovsky's site $C$ ) is identified as a cubic site because of the presence of only three transition lines. This identification is supported by our observation of a relatively long lifetime ( $10.2 \mathrm{~ms}$ ); radiative transitions of ions in cubic sites are by the less probable magnetic-dipole process. Detrio ${ }^{8}$ has calculated a magnetic-dipole oscillator strength of $6.35 \times 10^{-8}$ for this transition in $\mathrm{CaF}_{2}$, which implies a radiative time constant of $10.8 \mathrm{~ms}$; this is in good agreement with our observation.

Site 1 ions provided the most intense spectrum and have the lowest-energy Stark states. Makovsky identified this as a trigonal site (his site $T$ ) resulting from $0^{2-}$ compensation. He based this assertion on spectra obtained from samples grown under different conditions, from studying the effects of thermal treatment in vacuum and air, and on the EPR spectra of crystals undergoing thermal treatment observed by other. workers. 9 The large total Stark splitting $\left(313 \mathrm{~cm}^{-1}\right)$ and the significantiy lower center of gravity of the lavel indicate a relatively strong crystal-field interaction as might occur with this type of local charge compensation. However, our crystal was grown using modern techniquas that greatly reduce the amount of oxygen and other impurities incorporated into the lattice. This site is, therefore, tentatively identified as a $\mathrm{F}^{-}$ion compensated site and is probably the tetragonal (n.n.) site because of the large crystal-fieid interaction. 


\section{Vibronic Spectra}

Low-temperature $(20 \mathrm{~K})$ vibronic spectra of the different sites were obtained by exciting ions via transitions to their lowest-energy Stark state. The spectrum for site 1 is shown in Figure 4 along with the vibrational density of states (DOS) of pure $\mathrm{CaF}_{2}$ calculated by Elcombe and Pryor. 10 Vibronic spectra of the sites 2 and 3 included a great deal of structure that $w s$ due to zero-phonon transitions of other sites. This occurs because of the finite probability of excising an ion and simultaneously creating a phonon with a photon of frequency graater than its zero-phonon absorption frequency. The probability of this event is comparable to fluorescence with the emission of a phonon (i.e. a vibronic transition). Thus, when one excites ions of one site by zero-phonon absorption and observes fluorescence with emission of a phonon, one may also excite ions in ancther site with a lower-lying electronic level by absorption with the creation of a phonon; zero-phorion fluorescence from this other ion is of comparable intensity and overlaps the vibronic sidebands of ions in the first site. This process cannot uccur with site 1 ions because there are no electronic levels of ions in other sites linat are of lower energy. (Similar problems do not occur in glass spectra when excitation is in the low-frequency wing of the absorption/fluorescence band. Zero-phonon fluorescence from sites that ire excited by vibrationally-assisted absorption occurs only in the unmeasured low-frequency portion of the spectrum).

Structure in the vibronic spectrum of site 1 may be associated with vibrations of the pure $\mathrm{CaF}_{2}$ lattice (maximum frequency $=466 \mathrm{~cm}^{-1}$ ) plus an additional sharp peak at $560 \mathrm{~cm}^{-1}$ which we associate with motion of an interstitial $\mathrm{F}^{-}$ion. Three of the four bands in the DOS 


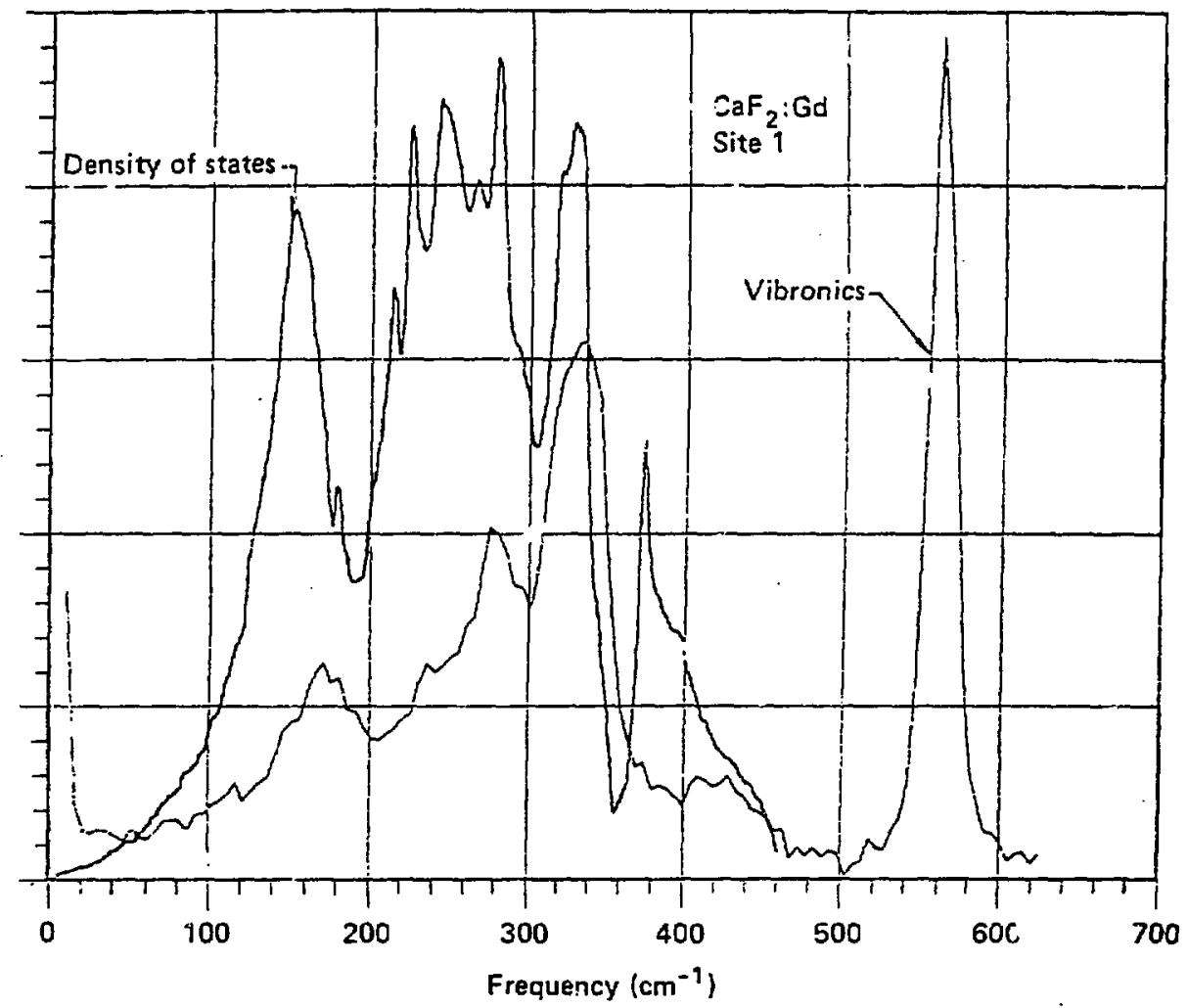

Figure 4: Vibronic spectrum of $\mathrm{Gd}^{3+}$ in $\mathrm{CaF}_{2}$ and the calculated vibrational density of states (after Elcombe and Pryor ${ }^{10}$ ). 
(occurring at 160,260 , and $325 \mathrm{~cm}^{-1}$ ) may be seen in the vibronic spectrum; vibronic selection rules apparently allow increased coupling to these vibration bands with increasing frequency. The fourth band in the DOS $\left(380 \mathrm{~cm}^{-1}\right)$ appears only weakly in the vibron is spectrum, indicating fairly strong matrix element effects. Elcombe and Pryor have indicated in the dispersion curves of vibrational modes for certain high-symmetry directions of the Brillouin zone that modes in the frequency range of the fourth band are predominantly longitudinal in character, while modes whose frequencies fall in the third band are predominantly transverse. These labels for the direction of motion of ions relative to the k-vector usually lose their meaning as one ventures from the high-symmetry directions into the wilds of the Brillouin zone. ${ }^{11}$ Also, the actual motion of ions about a point is similar regardless of the overall polarization of motion with respect to a $k$-vector. However, the correlation of the high-frequency vibronic band with "transverse" modes and not "longitudinal" modes is striking and can probably be explained by a difference in symmetry of these two types of modes.

The sharp high-frequency peak of the vibronic spectrum lies well above the frequency of any pure-lattice mode. The decay time constant of this peak was measured and found to be the same as the site 1 zero-phonon line $(2.3 \mathrm{~ms})$; this helps verify that the peak is actually a vibronic sideband. Its identification with the motion of the charge-compensating interstitial $\mathrm{F}^{-}$ion is supported by the fact that no line above the maximum lattice-phonon frequency was observed in the vithronic spectrum of the cubic site 3 . Thus, the mode is probably not due to a local mode resulting from only the presence of the triply-charged RE. If site $I$ is oxygen-compensated as asserted by Makovsky, ${ }^{7}$ a local mode could 
conceivably be created with in this frequency range.

Several workers ${ }^{12-14}$ have observed a sharp vibronic line in spectra of $4 f^{n} \rightarrow 4 f^{n-1}$ 5d transitions of REs in $\mathrm{CaF}_{2}$ at $490 \mathrm{~cm}^{-1}$. Th' explanations advanced as the origin of the mode are 1) motion if a tetragonal $\mathrm{F}^{-}$ion ${ }^{12}$ and 2) a localized "breathing" mode of the eight lattice $F^{-}$ians surrounding the RE. ${ }^{13,14}$ our work supports the first explanation. The differences in the observed local mode frequencies $(490$ vs. $560 \mathrm{~cm}^{-1}$ ) is probably due to the change in environment when ir the $4 f^{n-1} 5 d$ state. The bonding force-constants of the RE may be diff:rent for the excited state than for the $4 f^{n}$ ground state. A configurati nal coordinate model, in which the vibrational equilibrium positions of surrounding ions change with change in electronic state, is more appropriate for explanation of these interconfigurational vibronic transitions.

The vibronic results of site 1 present an opportunity to confirm the local RE-solid structure by vibrational spectroscopy in a manner analogous to the way molecular structure is confirmed. Modelling of the vibrational properties of the different possible charge compensation mechanisms should be straight-forward, especially in a material as vibrationally well-characterized as $\mathrm{CaF}_{2}$. The additional information provided by the vibronic spectrum should allow resolution of the question of oxygen or fluorine charge compensation.

In conclusion, we have seen an example of how laser-excited $\mathrm{Gd}^{3+}$ vibronic spectra can reveal information about vibrations and structure in RE-doped crystals. Further experimental and theoretical work along these lines should prove fruitfui. 


\section{References}

1. W. Hayes, ed., Crystals with the Fluorite Structure, Coxforo University Press, London, 1974).

2. J. Makovsky, Phys. Lett. 19647 (1966).

3. F. Z. Gil'fanov, L. D. Livanova, and A. L. Stolov, Sov. Phys. Solid State, 8108 (1966).

4. R. K. Watts, Point Defects in Crystals, (John Wiley, New York, 1977), p. 17.

5. J. A. Detrio, Phys. Lett. $28 \mathrm{~A} 82$ (1968).

6. W. Low, Phys. Rev. 109, 265 (1958).

7. J. Makovsky, J. Chem. Phys. 46390 (1967).

8. J. A. Detrio, Phys. Rev. 186339 (1969).

9. E. Friedman and W. Low, J. Chem. Phys. 331275 (1960).

10. M. M. Elcombe and A. W. Pryor, J. Phys. C: Solid State Phys. 3999 (1970).

11. P. L. Taylor, A Quantum Approach to the Solid State, (Prentice-Hall, New York, 1970), p. 96.

12. I. T. Jacobs, G. D. Jones, K. Zdansky, and R. A. Satten, Phys. Rev. B $32888(1971)$.

13. W. Hayes, M. C. K. Wiltshiu, W. J. Manthey, and D. S. McClure, J. Phys. C: Solid State Phys. $\underline{6}$ L273 (1973).

14. M. Schlesinger and W. F. Orake, Can. J. Phys. 541699 (1976). 
$-95-$

\section{Part III}

Orientational and Spectral Inhomogeneity in Nd:Laser Glass 


\section{Chapter 7: INTRODUCTION}

In a laser physics context, an inhomogeneous medium is one in which the individual optically active entities (atoms, ions, or molecules) present different cross sections to an incident beam of giveri frequency and polarization. Laser amplifiers based on inhomogeneous media perform differently than those with a homogeneous active material when operated in the large-signal "saturated" regime. ${ }^{1}$ for a qualitative understanding of the origin of saturation and the different behavior of homogeneous and inhomogeneous media, first consider the interaction of a thin slab of homogeneous laser material that has a population of identical excited ions and a temporally rectangular light pulse of intensity sufficient to de-excite a significant fraction of the ions. The change of intensity of the beam is $\sigma N I \delta x$, where $\sigma$ is the stimulated emission cross section of the transition (a quantity that measures the probability of interaction with light), $N$ is the population inversion density, I is the input intensity, and $\delta x$ is the thickness of the slab. ${ }^{2}$ The front edge of the rectangular pulse experiences intensity gain proportional to the initial population inversion but, in the process, de-excites ions. This decrease in $N$ causes subsequent portions of the pulse to be less amplified, resulting in an output puise that is distorted from the initial pulse. The time integral of the output intensity, i.e. energy, is a nonlinear function of the input energy. The gain in energy of the pulse increases linearly with increasing input energy for small inputs but approaches a constant value with large inputs. The slab is said to have been saturated when the energy gain approaches this value. 
Next, consider a slab of an inhomogeneous medium in which ions have a distribution of cross sections. Amplification of the front edge of the pulse is dominated by ions of large cross section (i.e. those with relatively high probability of interaction) and these ions are rapidly de-excited. The gain of subsequent portions of the pulse is determined by the remaining excited ions that have a continually decreasing average cross section. The shape of the amplified pulse depends on the details of the cross section and initial inversion distributions as we 11 as input intensity. Not only do the saturated pulse shapes of homogeneous and inhomogeneous systems differ; their energies also differ. Analys is reveals that the energy gain due to an inhomogeneous medium is always less than that due to a homogeneous medium with the same initial stored energy and average cross section. ${ }^{1}$

A great deal of theoretical work has been done on saturation in homogeneous short-pulse amplifiers. Bellman, Birnbaum and Wagner ${ }^{3}$ were the first to deal with the ideal two-level pulse amplifier. Soon afterwards, Frantz and Nodvik ${ }^{4}$ obtained closed-form solutions for output energy in terms of system parameters and input energy as well as expressions for output pulse temporal shape for two specific input pulse shapes. This treatment was extended to include effects of regeneration by Davis and Sooy ${ }^{5}$ and passive distributed losses by Avizonis and Grotbeck. ${ }^{6}$ Experimental verfication of the theory using short pulses in ruby rod amplifiers was successfully undertaken by several workers. 6,7

The treatment of inhomogeneous amplifiers has generally been restricted to steady state, continuous wave (CW) amplifiers in which enalytic solutions are obtainable for important inhomogeneity producing 
mechanisms. Spectral inhomogeneity in gases resulting from a Doppler shift of center frequencies is well known and the theory of saturation in CW gas amplifiers has been authoritatively treated by Gordon, White and Rigden. ${ }^{8}$ Spectral inhomogeneity in $\mathrm{CW}$ solid state amplifiers was dealt with by Seeber ${ }^{9}$ and more completely by Cabezas and Treat, ${ }^{10}$ with these workers allowing for the possibility of interaction of ions through cross-relaxation.

Theoretical studies of short-pulse inhomogeneous amplifiers have not appeared in widely disseminated literature, probably as a result of the inability to obtain analytic solutions for important models of inhomogeneity. The qualitative effects of inhomogeneity have been treated by Trenholme, 11 who has also developed an empirical "two-ion" model to predict saturation behavior in which the active medium is characterized by two populations of ions wit! different cross sections. 12,13 A thorough study of the two specific models of orientational and spectral inhomogeneity discussed in Chapter 9 was made by Haas. 14

The selective de-excitation of a class of ions in an inhomogeneous amplifier by a saturating light pulse may be observed in several ways. Perhaps the most heuristically pleasing method is to probe with a low-intensity light beam before and after the saturating pulse. Some characteristic of the probe beam relevant to the microscopic source of inhomogeneity is scanned and the selective de-excitation is revealed by a relative decrease in the probe's gain when its characteristic (e.g. wavelength, polarization) matches that of the saturating puise. The saturating pulse is said to have "burned a hole" in the small-signal gain 
profile of the medium. As an example, consider the phenomenon of spectral hole burning in a RE glass laser that results from a distribution of center frequencies of the ions' transitions. If a small-signal probe is scanned in frequency across the inhomogeneousiy broadened line, one finds that the ratio of post-pulse to pre-pulse gain of the probe will be less when the probe's frequency equals that of the saturating pulse. The width and depth of the hole in the relative gain profile depends on the ratio of homogeneous to inhomogeneous broadening in the line, the amount of saturation that takes place, and, in the case when rapid cross-relaxation is present, the time of observation after the saturating pulse.

Spectral hole burning in laser media can also be observed by monitoring the shape of the spontaneous emission spectrum before and after a saturating pulse. In a series of experiments, Nikitin, Soskin, and Khizhnyak ${ }^{15-17}$ observed ${ }^{4} F_{3 / 2} \rightarrow{ }^{4} I_{11 / 2}$ fluorescence spectra of $\mathrm{Nd}^{3+}$-doped glasses before and after exposure to a "giant", frequencytunable pulse. They found a single dip in the spectrum of each sample at the wavelength of the saturating pulse $(1.06 \mu \mathrm{m})$ indicating hole burning. The width and depth of the burned hole was found to vary with host composition, temperature (which affects a transition's homogeneous width), and saturating pulse bandwidth. Other methods of verifying spectral inhomogeneity in $\mathrm{Nd}$ : laser $\mathrm{glass,}$ which involved time-resolved spectra of the buildup of oscillation in a laser cavity, were employed by Snitzer 18 and Keane and Weiss. 19

Recent experiments by Martin and Milam ${ }^{20-22}$ using the amplifierprobe method, in addition to confirming spectral hole burning, also 
indicate an additional source of cross section inhomogeneity. In these experiments, a large linearly polarized saturating pulse was sent through a pumped amplifier while small-signal gain was monitored by a polarized CW probe propagating approximately collinearly with the saturating pulse. Comparison of gain of the probe with polarization parallel and perpendicular to that of the saturating pulse revealed less post-pulse gain' in the parallel configuration. In other words, the saturating pulse burned a polarization dependent hole in the medium's gain profile.

An explanation of these resuits, proposed by krupke 23 and investigated by Haas, ${ }^{24}$ was based on an assumed cross section anisotropy of Nd ions in glass. Although the Nd sites themselves are isotropically oriented, an individual site environment may cause an ion to have a preferred direction for interaction with a polarized beam. Those ions with their preferred direction oriented along the polarization of the saturating pulse would be selectively de-excited while those perpendicular to the pulse would be less affected.

As discussed in Chapter 2, one cannot tel1 by standard sma11-signal absorption and fluorescence measurements if a system is homogeneous or inhomogeneous. For example, one does not know if a broad band in an absorption spectrum is due to a collection of identical absorbing sites each with a large homogeneous width or sites with narrow lines whose line-centers are distributed about the observed band's peak. Analogous results hold for a possible polarization inhomogeneity. Usually, evaluation of materials for glass laser amplifiers has been based solely on this type of small-signal experiment. ${ }^{25,26}$ As designs for fusion 
drivers and other applications push for greater and more complete energy extraction, knowledge of all inhomogeneities becomes critical for efficient operation.

To verify and quantify a cross section anisotropy in $\mathrm{Nd:glass} \mathrm{laser}$ material, we preformed a different type of small-signal experiment using the polarized, fluorescence line-narrowing (FLN) technique. A resonant FLN experiment may be thought of as the inverse of a laser gain experiment in that ions are selectively excited from the terminal laser level and their fluorescence observed instead of the selective de-population of the upper laser level that occurs in an amplifier. The resonant character of the experiment is necessary to restrict the response solely to those ions that would be de-excited by a saturating pulse in an amplifier (see Chapter 2).

The first observation of polarization effects of a rare earth in glass ( $\mathrm{Eu}^{3+}$ in calcium metaphosphate) using the polarized FLN technique was made by Kushida, Takushi, and Oka. ${ }^{27}$ Lebedev and Przhevuskij ${ }^{28}$ later applied the technique to a variety of ions in several amorphous hosts, using both resonant and nonresonant transitions.

The FLN experiment has the advantage of being, in essence, a small-signal experiment without the initial condition (pumping stability, intensity profile) and integration (growth of signal with distance) problems of an amplifier gain measurement. Also, in the context of optimization of material parameters, a FLN experiment requires on.ly a small sample with fairly constant apparatus configuration as opposed to requiring a full-sized operating device. 
Unpolarized FLN experiments have successfully identified the spectrally inhomogeneous nature of the usual lasing transition of Nd:glass. ${ }^{29}$ In an analogous way, the observation of a polarized FLN effect, in the absence of cross-relaxation, is necessary and sufficient to predict polarization hole burning in laser amplifiers.

The apparatus, procedure, and results of polarized FLN experiments performed on two Nd:silicate laser glasses are presented in the next chapter.

To analyze the macroscopic results of the polarized FLN experiment and to predict amplifier preformance, a microscopic model of the Nd ion in glass in necessary. A simple phenomenological model for polarization inhomogeneity is presented in Chapter 9 and used to relate FLN experimental results with microscopic parameters. Next, after a treatment of the influence of a general, unspecified inhomogeneity on amplifier performance, calculations are performed for an amplifier based on a medium made up of the model sites. The effect of spectral inhomogeneity on short-pulse amplifier performance is examined next by assuming a simple distribution of homogeneous lines. Then both spectral and polarization inhomogeneities are included in an amplifier calculation to produce a realistic model of a short-pulse Nd:glass laser with parameters obtainable from small-signal and FLN experiments.

Finally, in Chapter 10 , the results of these calculations are compared with the experimental results of Martin and Milam. The limitations and caveats necessary for this simple model to be applicable to a real laser medium are discussed. 


\section{References}

1. M. 3. Weber, 3. Non-Crystalline Solids 47, 117 (1982).

2. See, e.g., W. Koechner, Solid-State Laser Engineering, (Springer, Berlin, 1976) p. 16.

3. R. Bellman, G. Birnbaum and W. G. Wagner, J. App 1. Phys. 34,780 (1963).

4. L. M. Frantz and J. S. Nodvik, 3. App1. Phys. 34, 2346 (1963).

5. J. I. Dayis and W. R. Sooy, App1. Optics $\underline{3}, 715$ (1964).

6. P. V. Avizonis and R. L. Grotbeck, J. App 1. Phys. 37, 687 (1966).

7. E. L. Steele and W. C. Davis, J. Appl. Phys. 36, 348 (1965).

8. E. I. Gordon, A. D. White, and J. D. Rigden, Proc. of the Symposium on Optical Masers 1963, (Polytechnic Press, New York) p. 309.

9. K. N. Seeber, IEEE Transactions ED-2 63 (1965).

10. A. Y. Cabezas and R. P. Treat, J. Appl. Phys. 37, 3556 (1966).

11. J. Trenholme, LLL Memo SSLP 79-29, Nova File 1.2.1

12. J. Trenholme, LLL Laser Program Annua] Report 1979, UCRL-50021-79 $2-160$.

13. J. Trenholme, LLL Laser Program Annual Report 1980, UCRL-50021-80 2-193.

14. R. Haas (unpublished).

15. V. I. Nikitin, M. S. Soskin, and A. I. Khizhnyak, Sov. Tech. Phys. Lett. ?;, 64 (1976).

16. V. I. Nikitin, M. S. Sos'kin, and A. I. Khizhnyak, Sov. Tech. P ys. Lett. $\underline{3}, 5$ (1977). 
17. V. I. Nikitin, M. S. Soskin, and A. I. Khizhnyak, Sov, J. Quant. Elect. $\underline{8}, 788$ (1978).

18. E. Snitzer, Proc. 3rd Quant. Elect. Conf. (1964) p. 999.

19. W. H. Keene and J. A. Weiss, App 1. Opt. 3, 545 (1964).

20. W. E. Martin and D. Milam, App T. Phys. Lett. 32, 816 (1978).

21. W. E. Martin and D. Milam, LLNL UCID Report 18868 (1980).

22. W. E. Martin and D. Milam, J. Quant. Elect. (to be submitted).

23. W. F. Krupke, LLL Memo WFK 78-100 (August 1978).

24. The unpublished theoretical development and results of calculations were readily made available to the author.

25. E. Snitzer, Appl. Optics $\underline{5}, 1487$ (1966).

26. C. G. Young, IEEE Proc. I, 1267 (1969).

27. T. Kushida, E. Takushi, and Y. Oka, J. Lumin. 12/13, 723 (1976).

28. V. P. Lebedev and A. K. Przhevuskii, Sov. Phys. So iid State 19, 1389 (1977).

29. S. A. Brawer and M. J. Weber, Appl. Phys. Lett. 35, 31 (1979). 
Chapter 8: EXPERIMENTAL DETAILS AND RESULTS

A polarized FLN experiment, configured in the usual $90^{\circ}$ excitationobservation geometiy of Figure 1, consists of illuminating a sampie with a pulse of linearly polarized monochromatic light and measuring the resonant or nonresonant fluorescent intensity with the axis of a polarization analyzer parallel (I") and perpendicular (I ${ }^{\perp}$ ) to the polarization of the exciting light. The ratio of these intensities, $p=$ I'/I", is called the depolarization ratio after the analogous quantity found in molecular and Raman scattering.

When this conceptualiy simple technique is used to study the ${ }^{4} \mathrm{~F}_{3 / 2} \rightarrow{ }^{4} \mathrm{I}_{11 / 2}$ transition of $\mathrm{Nd}^{3+}$, however, both intrinsic and pragmatic factors cause difficulties, usually manifesting themselves in low signal levels. This No transition is useful as a laser transition because its terminal level is $2000 \mathrm{~cm}^{-1}$ above the ${ }^{4} \mathrm{I}_{9 / 2}$ ground state and is unpopulated in equilibrium, except for the small fraction of ions dictated by the Boltzmann factor $\left(<10^{-4}\right.$ at $\left.300 \mathrm{~K}\right)$. It is these few thermally-excited ions that participate in a $1.06 \mu \mathrm{m}$ excited resonant FLN experiment. A practical problem that affects signal strength is the lack of an efficient low-intensity photodetector at this wavelength. 1 The best commercially available photocathode (S-1) has a nominal quantum efficiency (when given) of $.03 \%$ at $1.06 \mu \mathrm{m}$.

A practical problem that necessitates modification of the experimental scheme of Figure 1 is the polarization dependence of spectrometer grating efficiency. This dependence creates a detection bias that is difficult to account for by normalization. For example, the ratio of horizontal to 


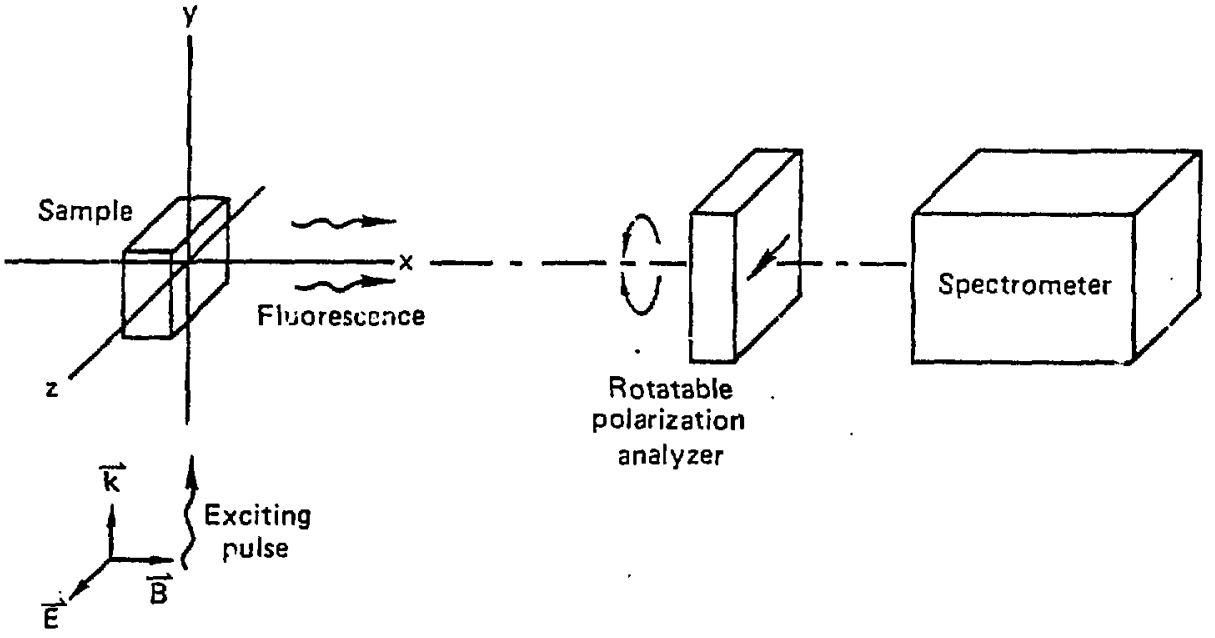

Figure 1: Usual 900 excitation-observation geometry for polarized fluorescence line-narrowing experiments. The $I^{-1}$ and I". signals are obtained by rotating the polarization analyzer. 
vertical throughout for one high-efficiency grating was found to be $\approx 5$ at $1.06 \mu \mathrm{m}$. Although pseudopolarization scramblers exist to convert linearly polarized light into randomly polarized light, no combination of scrambler and grating was found to reduce this bias to an acceptable level. Also, any method of mixing the polarization (e.g. scrambler, $\frac{1}{4}$ plate) puts some signal energy into a low efficiency polarization, decreasing throughout.

The bias problem can be circumvented by allowing only light with polarization along the $Z$ axis to enter the spectrometer and by rotating the polarization of the exciting beam from the $Z$ direction ( $I$ " signal) to the $x$ direction ( $I^{\perp}$ signal). The $I "$ signal is, of course, the same as that of Figure 1 , but the equivalence of the two $I^{\perp}$ signals requires an assumption of $i$ ) an isotropic medium and $i j$ ) electric-dipole transitions. To see this equivalence, ${ }^{2}$ realize that. if the signal polarized along $Y$ were from a dipole radiator, it would be the same anywhere in the $x-z$ plane at a fixed distance from the origin because dipoles radiate symetrically about their axes. Specifically, the $Y$-polarized intensity along the $X$-axis $\left(I^{\perp}\right)$ equals the $Y$-polarized intensity along the $Z$-axis. This latter quantity is just that wh $\ldots$ is measured by rotating the polarization of the exciting beam by $90^{\circ}$ (so that it points toward the spectrometer), if the sample is isotropic. The first assumption is justified by the amorphous nature of the host, while the second is justified for the same reason a "forbidden" transition has such a relatively high oscillator strength: the admixing of 50 configuration states by the crystal field. ${ }^{3}$ 
Details of the experiment are discussed within the categories of sample properties, excitation beam characteristics, and observation optics and electronics. Procedures for recording data, and results for resonant $\left({ }^{4} \mathrm{I}_{11 / 2} \rightarrow{ }^{4} \mathrm{~F}_{3 / 2}:{ }^{4} \mathrm{~F}_{3 / 2} \rightarrow{ }^{4} \mathrm{I}_{11 / 2}\right)$ and nonresonant $\left({ }^{4} \mathrm{I}_{11 / 2} \rightarrow{ }^{4} \mathrm{~F}_{3 / 2}:{ }^{4} \mathrm{~F}_{3 / 2} \rightarrow{ }^{4} \mathrm{I}_{9 / 2}\right)$ experiments are then presented with mention of possible sources of error. Finally, these results are discussed vis-a-vis other RE:glass measurements.

\section{A. Samples}

The two Nd:silicate glasses studied were ED-2 (formerly Owen-Illinois, now Schott LG-670) and LG-650 (Schott), both commercially available products. Dimensions of samples were about $2 \times 2 \times 1 \mathrm{~cm}$ with all faces polished to optical quality.

\section{Broadband excited ${ }^{4} F_{3 / 2} \rightarrow{ }^{4} I_{11 / 2}$ fluorescence spectra for} these glasses is shown in Figure 2 and useful spectroscopic data is given in Table I. The stimulated emission cross sections were derived from small-signal spectroscopic measurements using the Judd-Ofelt model. ${ }^{4}$ The effective linewidth of the ${ }^{4} F_{3 / 2} \rightarrow{ }^{4} I_{11 / 2}$ transition, $\Delta \nu_{\text {eff }}$, is defined as the wavelength integral of broadband excited fluorescence intensity divided by the value of the peak intensity. Also included in the table is a value for the homogeneous linewidth of Nd:ED-2 obtained from FLN experiments ${ }^{5}$ and the measured values of $\rho$.

The Nd doping of both samples was at a level where ion-ion energy transfer ${ }^{5}$ was negligible on the time scale of observation $(\simeq 2$ e-folding times). A higher $N d$ concentration is permissable in LG-650 than in ED-2 because of smaller dipole-dipole interaction between the ions; the 
smaller dipole moment of the transition also results in a lower cross section. These differences are a result of the dissimilar modifier cation composition of the glasses, with LG-650 made up of larger, more polarizable modifiers.

Table 1. Small-signal spectroscopic and doping data of the two silicate glasses used in $\mathrm{Nd}^{3+}$ depolarization experiments.

\begin{tabular}{lcc}
\hline & $\frac{E D-2}{1.061}$ & $\frac{L G-650}{1.057}$ \\
$\nu_{\text {peak }}(\mu \mathrm{m})$ & 304 & 303 \\
$\Delta \nu_{\text {eff }}\left(\mathrm{cm}^{-1}\right)$ & 309 & 430 \\
1st e-folding time, $(\mu \mathrm{s})$ & 2.7 & 1.1 \\
$\sigma\left(\mathrm{pm}^{2}\right)$ & $25^{(\mathrm{a})}$ & -- \\
$\Delta \nu_{\mathrm{h}}\left(\mathrm{cm}^{-1}\right)$ & 1.5 & 5 \\
$\mathrm{Nd}_{2{ }^{3}{ }^{3} \text { doping (wt.\%) }}$ & 1.37 & 4.70 \\
$\mathrm{Nd}^{3+}$ concentration $\left(10^{20}\right.$ ions/cc) & $0.89 \pm 0.03$ & $0.88 \pm 0.06$ \\
$p$ & & \\
\hline
\end{tabular}

Source: S. E. Stokowski, R. A. Saroyan, and M. J. Weber, "Nd:doped Laser Glass Spectroscopic and Physical Properties", Lawrence Livermore National Laboratory, Report M-095 (2nd revision) 1981.

(a) S. A. Brawer and M. J. Weber, Appl. Phys. Lett. 35,31 (1979). 

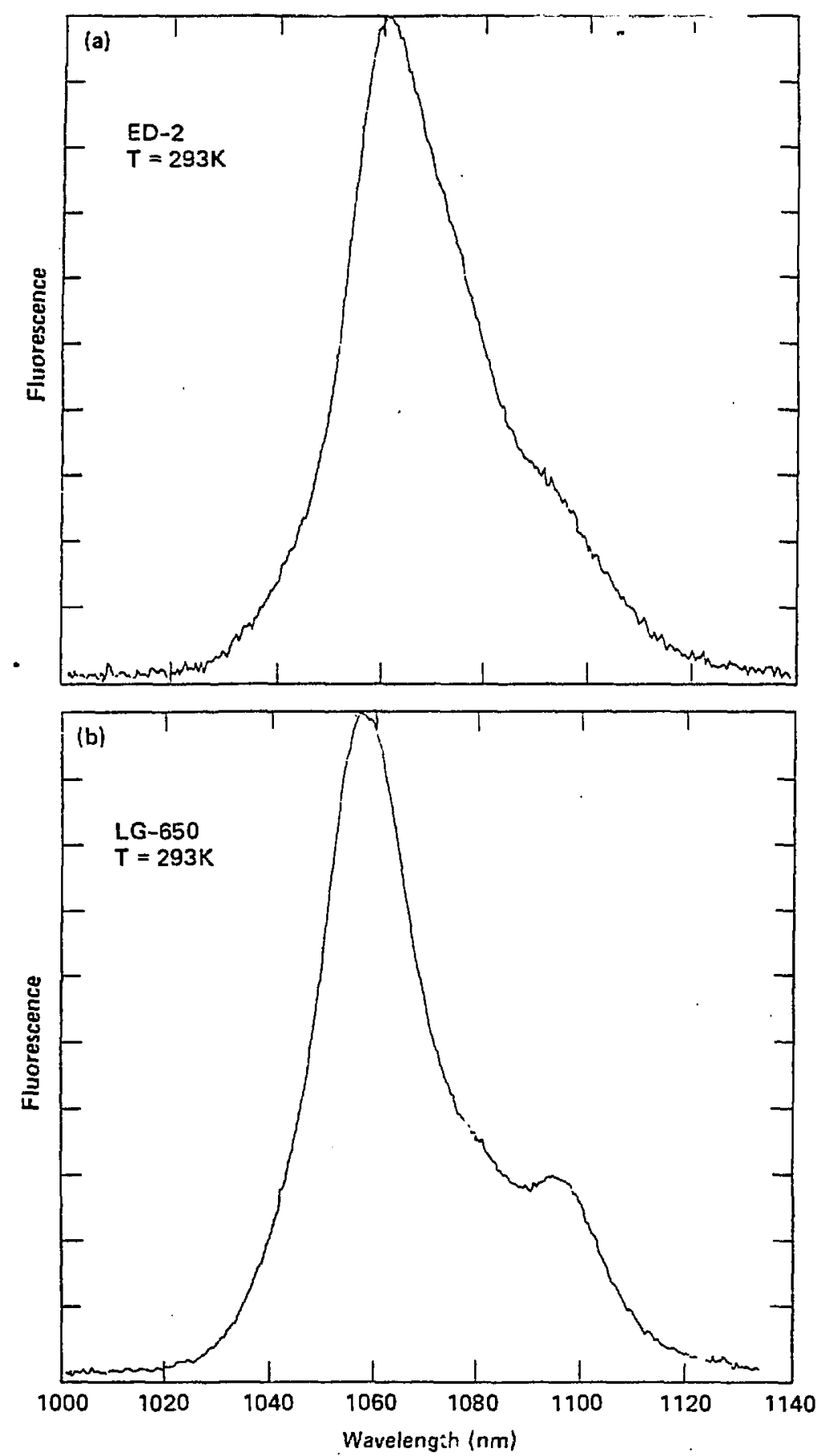

Figure 2: Broadband excited ${ }^{4} F_{3} \rightarrow{ }^{4}{ }^{4}$ fluorescence of $\mathrm{Nd}^{3+}$ in a) ED-2 and b) LG-650 sî4 cate glasses. 


\section{B. Excitation}

The excitation source and beam controlling optics are shown schematically in Figure 3. A $40 \mathrm{~ns}, 1.064 \mu \mathrm{m}$ pulse was generated by a Q-switched Nd:YAG Oscillator/amplifier system (Molectron MY34-20) with a spectral linewidth of $<0.5 \mathrm{~cm}^{-1}$. The laser was operated at $10 \mathrm{~Hz}$ and produced a beam of $\approx 1 \mathrm{~cm}$ diameter. Polarization of the output beam was vertical and linear polarization was assured by the addition of a Glan prism polarizer (GI in Figure 3a). The beam was directed by $1.06 \mu m$ dielectric mirrors.

The average laser power was monitored by using a beamsplitter (parallel-face glass plate yielding $\approx 8 \%$ reflection) and a radiometric power meter (Scientech 361). Typical average power after the beamsplitter was $0.7 \mathrm{~W}$ implying $70 \mathrm{~mJ} / \mathrm{pulse}$.

The beam was then passed through a $1-m$ focal length lens, Ll, (set with focus $20 \mathrm{~cm}$ beyond sample) and turned into a "dark box" enclosure by a dielectric front coated mirror. Inside the box, it was turned again (Figure $3 b)$ so that the beam was propagating in a vertical direction, which allowed for optimum imaging of the fluorescence streak in the sample onto the vertical slits of the spectrometer. Another Glan prism (G2) ensured linear polarization of the beam along the $Z$ direction.

The final optical component before the sample was a $1.06 \mu m$ half-wave plate mounted to rotate the direction of polarization in the $X-Z$ plane. The beam traversed the plate coaxially and therefore passed through the same volume regardless of rotation. Also, rotation of the plate did not 
(a) Side view

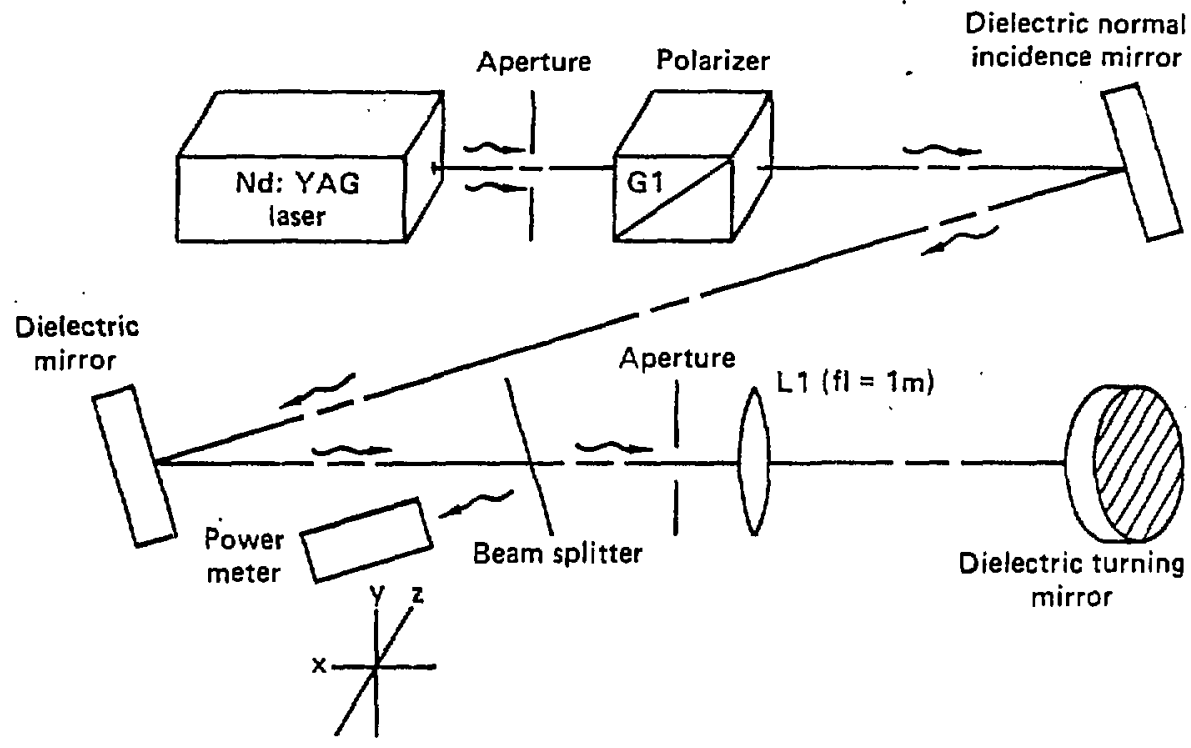

(b) End view

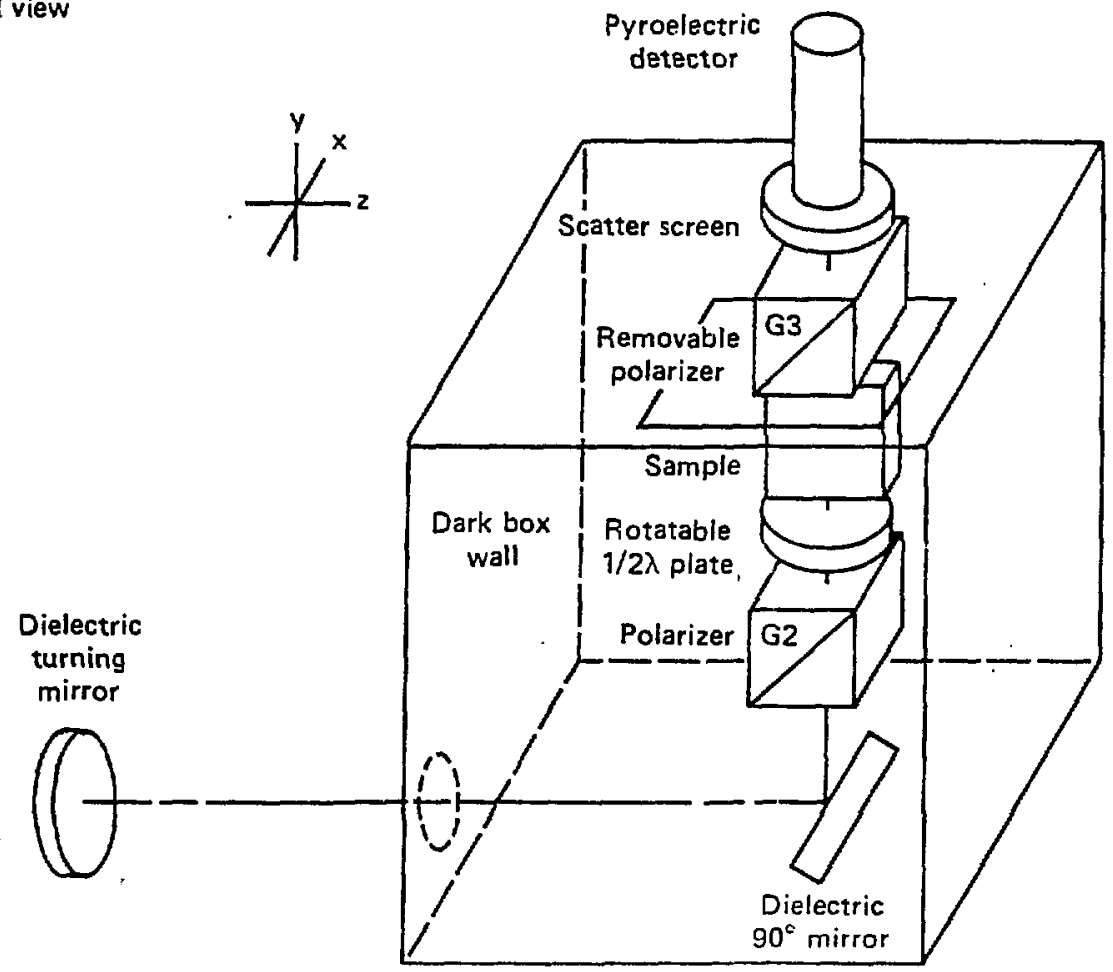

Figure $3:^{*}$ Schematic diagram of the excitation source and optics used in $\mathrm{Nd}^{3+}$ polarized FLN experiments: a) side view; b) end view. 
steer the beam, as was verified by observing the beam several meters away from the plate. The actual polarization of light passing through the sample was determined using a removable Glan prism (G3). This polarizer was set so that its transmitting orientation was perpendicular to the desired direction of polarization and then the half-wave piate was rotated until a null was achieved (using a Kodak IR Phosphor card as detector). .

At the sample, the beam was weakly convergent with $\simeq 2 \mathrm{~mm}$ diameter and well-characterized linear polarization.

During a measurement, the Glan prism (Gaj) was removed and the energy/pulse of the exciting bean monitored with a pyroelectric energy meter (Laser Precision Rj-7200). The sensitive absorbing surface of this detector required a scatter screen to attenuate the beam, precluding absolute energy readings. The average beam power after the sample, measured with a power meter (Coherent 210), was consistent with that measured after tine beamsplitter when Fresnel losses of interposed components were considered. Variations in average energy of $<1 \%$ in 5 minutes were observed with the pyroelectric deiector and were correlated with drifts in laser autput measured with the Scientech power meter. These dritts were accounted for in the data reduction. C. Observation

The observation optics train is shown in Figure 4 . The vertical fluorescence streak in the sample was imaged onto the entrance slit of a $1-\mathrm{m}$ grating spectrometer (Chromatix CT-103, blaze $=0.5 \mu \mathrm{m}, 1200 \mathrm{lines} / \mathrm{cm}$ ) using a fast collection lens $(f t=4 \mathrm{~cm}, f / 1.1\}, L 2$, and a lens $\{f l=15 \mathrm{~cm}$, f/7), L3, consistent with the spectrometer's effective speed. 


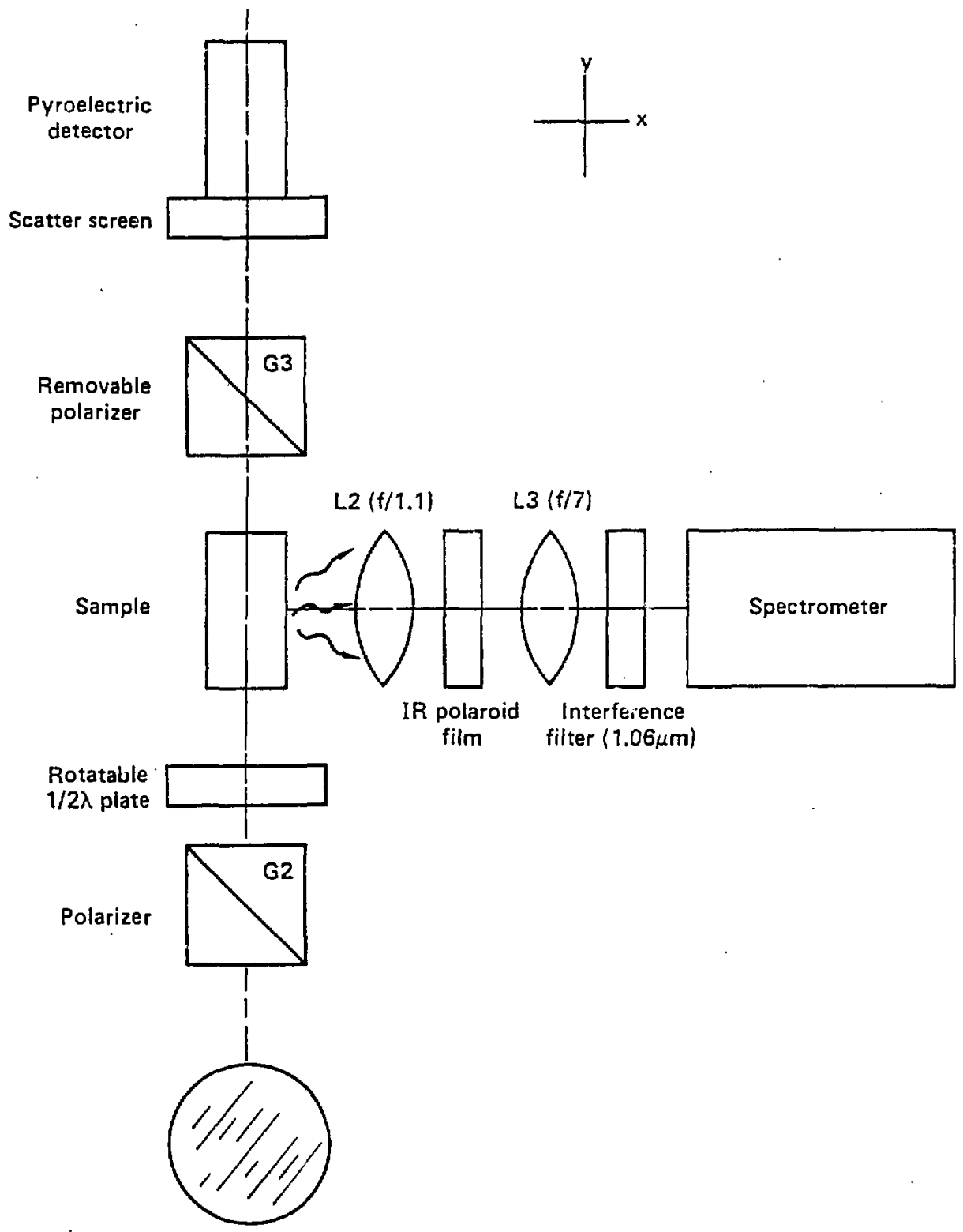

Figure $4:-$ Schematic diagram of the observation optics train used in $\mathrm{Nd}^{3+}$ polarized FLN experiments. 
An IR Polaroid film polarizer (Oriel 3959) was placed between these lenses to allow only $z$-polarized light to reach the spectrometer. The film was chosen over other types of polarizers because of its large aperture and angle of acceptance. A polarizer's extinction coefficient, $\boldsymbol{\alpha}$, is defined as the ratio of transmission of unpolarized light through two identical polarizers with axes crossed to the transmission measured with axes parallel (a Glan prism typically has an $\alpha=10^{-5}$ ). The measured extinction coefficient of the film was $<10^{-2}$ at $1.06 \mu \mathrm{m}$.

A $1.06 \mu \mathrm{m}$ interference filter (peak transmission $78 \%, F W H M=180 \mathrm{~cm}^{-1}$ ) was places in front of the entrance slits of the spectrometer when measuring resonant $1.06 \mu \mathrm{m}$ fluorescence. This was necessary because 1 ight from the nonresonant ${ }^{4} \mathrm{~F}_{3 / 2} \rightarrow{ }^{4} \mathrm{I}_{9 / 2}$ transition (peaked at $880 \mathrm{~nm}$ ) is also focused onto the entrance slit. Usually a spectrometer acts as an effective filter for unwanted spectral components. In this experiment, however, the small number of scattered $880 \mathrm{~nm}$ photons that reached the detector, coupled with the relatively large quantum efficiency at this wavelength $(\approx 0.3 \%, 10$ to 100 times that at $1.06 \mu \mathrm{m})$, created a significant background problem. The interference filter eliminated this spectral. component.

For resonant $1.06 \mu \mathrm{m}$ measurements, the spectrometer was operated with a. $3 \mathrm{~mm}$ entrance and exit slit width resulting in a pass band of $\simeq 25 \mathrm{~cm}^{-1}$ at $1.06 \mu \mathrm{m}$. Increased sensitivity for ${ }^{4} \mathrm{~F}_{3 / 2} \rightarrow{ }^{4} \mathrm{I}_{9 / 2}$ fluorescence allowed slit widths of $1 \mathrm{~mm}$, yielding a $10 \mathrm{~cm}^{-1}$ pass band for nonresonant spectra. The slit height was $1 \mathrm{~cm}$.

Figure 5 is a block diagram of the detector and signal processing 


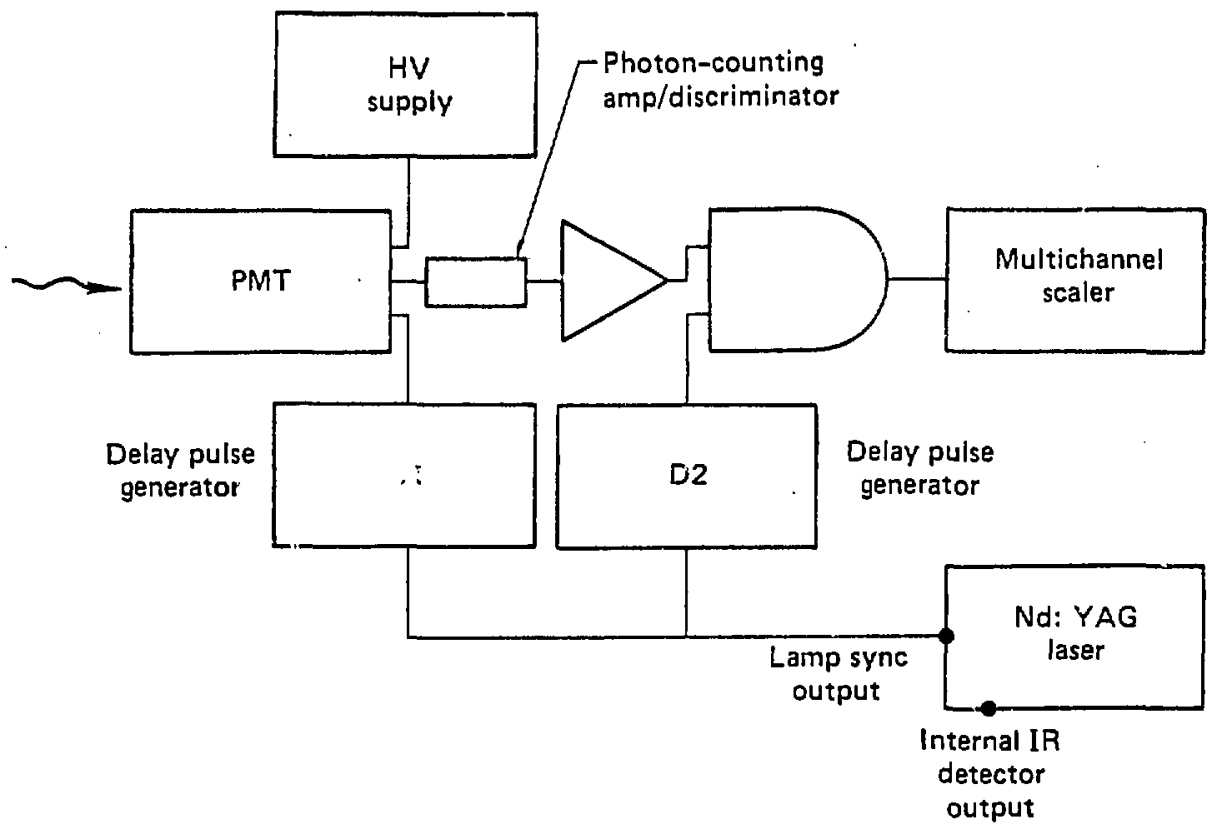

Figure 5: Block diagram of the detector and signal processing electronics used in $\mathrm{NA}^{3+}$ polarized FLN experiments. 
electronics. The detector wâs a dry ice chillen $\$$ I photomultiplier (EMI 9808B) operated in the photon-counting mode. The photon-counting amplifier/discriminator (PAR model 1121) was set with a discriminator threshold of $2.0 \mathrm{mV}$ which gave an optimum signal/noise vs. signal throughput value at the PMT bias voitage of $1.8 \mathrm{kV}$. This threshold level and the chillirig of the tube resulted in a very low dark count rate $\left(=20 \mathrm{~s}^{-1}\right)$. The background during resonant experiments ( $v i$ th the interference filter in place) was accounted for by this dark count. A pulse conditioning operational amplifier converted the output of the amplifier/discrininator to TTL compatible levels.

The large number of scattered photons from the exciting laser also caused problems during, resonant measurements by saturating the PMT and counting electronics, producing unreliable count rates unt $i 1>200 \mu \mathrm{s}$ after the exciting pulse. By this time the fluorescence intensity would decay to $\simeq e^{-2 / 3}$ of its initial value so that normal gated-photoncounting techniques "wasted" much of the potential signal. This problem was Eliminated by gating the first cynode of the PMT (EMI circuit GB1001A), i.e. the potential difference between the cathode and first accelerating dynode was turned off during the laser pulse, making the tube temporaril, inactive.

A schematic diagram of relevant timing signals is shown in Figure 6 . The master timing pulse was generated by the laser's LAMP SYNC output which triggered $180 \mu$ s before the laser's Q-switch fired. Timing for the dynode-gate circuit was provided by a pulse/delay generator (Tektronix 
Laser lamp sync

Laser IR detector

Dynode gate

Photon counting gate

Ampldisc. output

Pulses to scaler

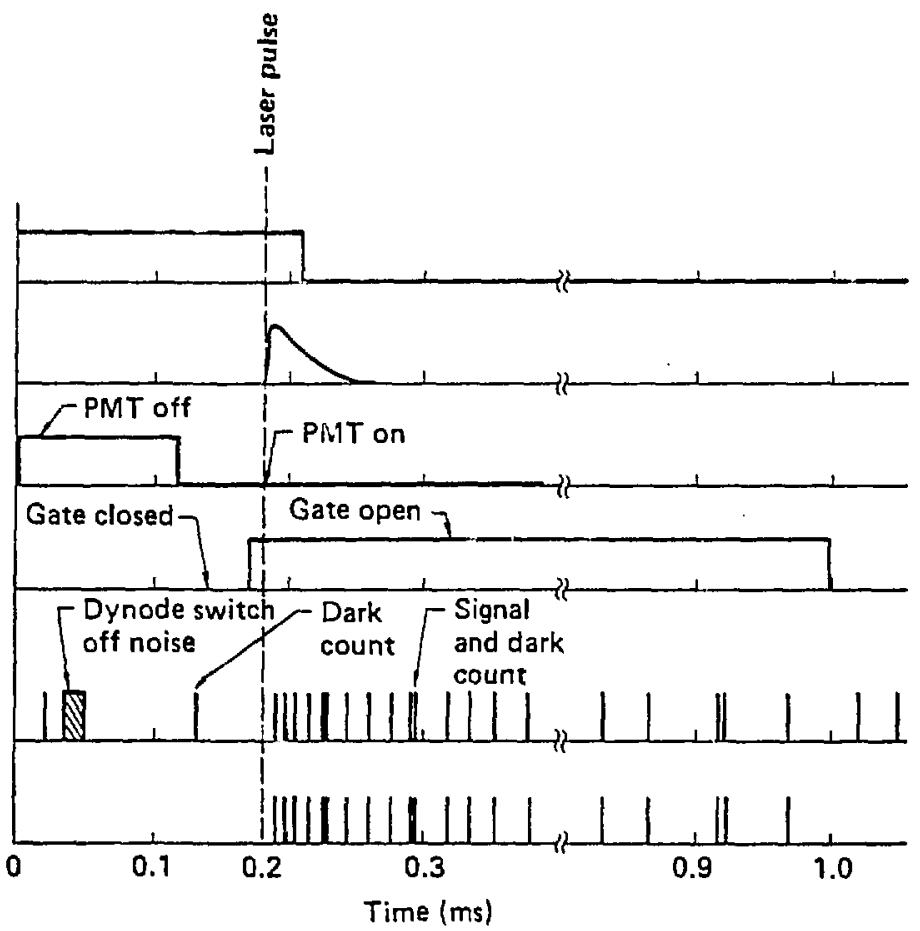

Figure 6: Schematic diagram of timing signals and photon counting pulses used in detection of $\mathrm{Nd}^{3+}$ fluorescence. 
26G3), 01. The switch-on time of the dynode-gate is nominally $5 \mu \mathrm{s}$, but there is an $\simeq 70 \mu$ s delay within the circuit between arrival of the timing signal and the start of PMT activation. Hence, the dynode-gate control signal was set to turn the tube on (Logic $\phi$ level) $60 \mu$ s before the laser pulse. Also, a significant noise pulse was generated when the dynode was originally switched off. This was eliminated by the photon-counting gate (see below). The time at which the Q-switch fired and the laser puise was generated was monitored by an IR power meter internal to the laser, which has a significant RC time constant.

Photon-counting of fluorescence for longer than a few e-folding times is not usefu? since by then the signal is small and counts are mostly from noise. A photon-counting gate which limits the collection time of signar purses was included by putting the pulses and a timing signal generated by 02 into an AND logic circuit. The counting gate was set to collect pulses for $600 \mu$ s after the laser pulse with ED-2 and $800 \mu$ s with LG-650. The output of the AND circuit was then accumulated by a multichannel scaler (Tracor-Northern NS-570). For single wavelength measurements, counts were accumulated in a single scaler. When a spectrum was desired, the scaler channel was incremented at a rate proportional to the spectrometer scan rate. Data was then transferred to an LSI-11 microcomputer for display on a graphics terminal (Tektronix 4010) and storage on floppy disk. 


\section{Procedure and Results}

Resonant $1.06 \mu$ m measurements

When measuring the signal for a given excitation beam polarization, the background count rate was first determined. The background at $1.06 \mu \mathrm{m}$ was determined by interpolation of background measured at $1.020 \mu \mathrm{m}$ and $1.140 \mu \mathrm{m}$, wavelengths outside of the ${ }^{4} \mathrm{~F}_{3 / 2} \rightarrow{ }^{4} \mathrm{I}_{11 / 2}$ fluorescence band (see Figure 2). Counting time for a background data point was 180 seconds (1800 raser shots) yieiding a total count of $\simeq 30$ when using a photon counting gate of $800 \mu \mathrm{s}$. Background count rates at both measured wavelengths were equal and found to have no polarization dependence, within statistical uncertainty. The spectrometer was then set $a^{2} 1.064 \mu m$ and data collected for $\simeq 400$ seconds (total count of $\simeq 1000$ ). The pyroelectric detector electronics averaged the energy of every 100 laser shots and this average was recorded. The 40 averages recorded per measurement were also averaged so that the energy of each shot was accounted for. This second average value was used to obtain normalized count rates and never varied by more than $1 \%$ during a 400 second counting period.

Next, polarization of the exciting beam was rotated $90^{\circ}$ by a $45^{\circ}$ turn of the half-wave plate (and checked using the removable Glan prism, G3) and background and data counting was repeated. The half-wave plate was always rotated in one direction to minimize undetected beam steering effects.

Normalized count rates minus normalized background rates were then averaged to yield averaged values of $I^{\perp}$ and $I^{\prime \prime}$. These were divided to give $\rho$. For $E D-2, \rho=0.89 \pm 0.03$ and for $L G-650, \rho=0.88 \pm 0.06$. 
Estimates of error represent the standard deviation of six measurements of $I^{\perp}$ and $I^{\prime \prime}$ and the propagation of error resulting from taking the ratio of two uncertain quantities. The larger standard deviation of the LG-650 measurement results from a lower signal count rate and higher background count rate (the photon-counting gate was open longer). Values of depolarization calculated without using average power normalization differed by $1 \%$ or less.

Nonresonant measurements

A peak value of the depolarization ratio of the ${ }^{4} F_{3 / 2} \rightarrow{ }^{4} I_{9 / 2}$ transition with ions excited at $1.064 \mu \mathrm{m}$ was measured and gave $\rho=0.92 \pm$ 0.02 for ED-2 $(882 \mathrm{~nm})$ and $\rho=0.96 \pm 0.02(880 \mathrm{~nm})$ for $L G-650$. The increased detector sensitivity in this wavelength region allowed polarized spectra to be measured in a relatively short time (10 minutes). These spectra are shown for ED-2 in Figure 7 and for LG-650 in Figure 8. Error limits represent the uncertainty occurring from a finite number of counts $(\sqrt{N})$.

\section{E. Systematic Error}

The precision of depolarization measurements was fealt with in the preceeding section using the usual analysis of statistically varying quantities. ${ }^{6}$ Questions of accuracy, which imply unknown systematic error, need to be addressed.

The experimental apparatus and procedure were designed to be as constant as possible for measurement of $I^{\perp}$ and I", i.e. only the polarization of the incident beam was changed.

Confidence in the lack of systematic error in the more important 


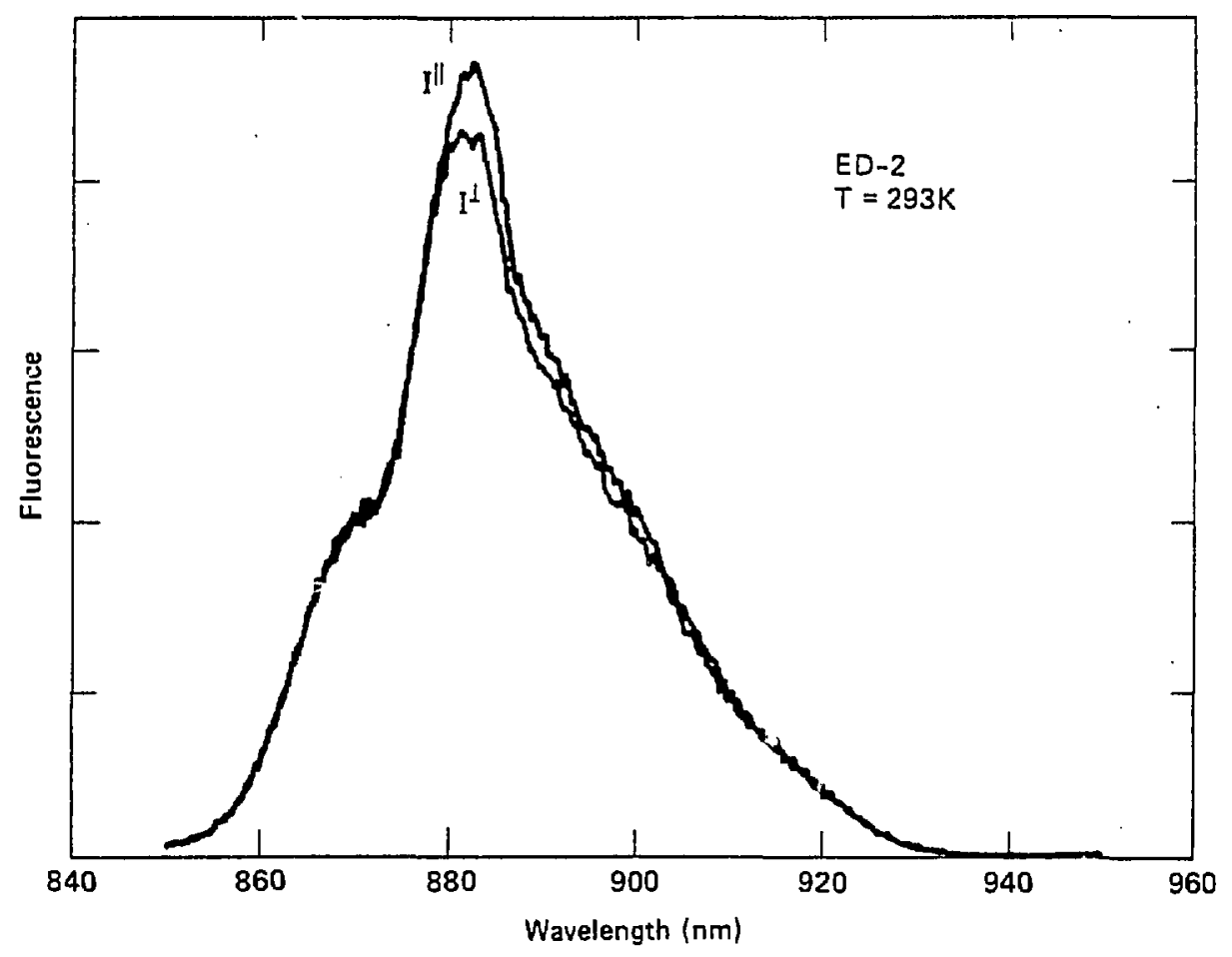

Figure 7: Polarized fluorescence spectra of the $\mathrm{Nd}^{3}+{ }^{4} \mathrm{~F}_{3 / 2} \rightarrow{ }^{4} \mathrm{I}_{9 / 2}$ transition in ED-2 silicate glass excited by $1.064 \mu \mathrm{m}$ laser light. 


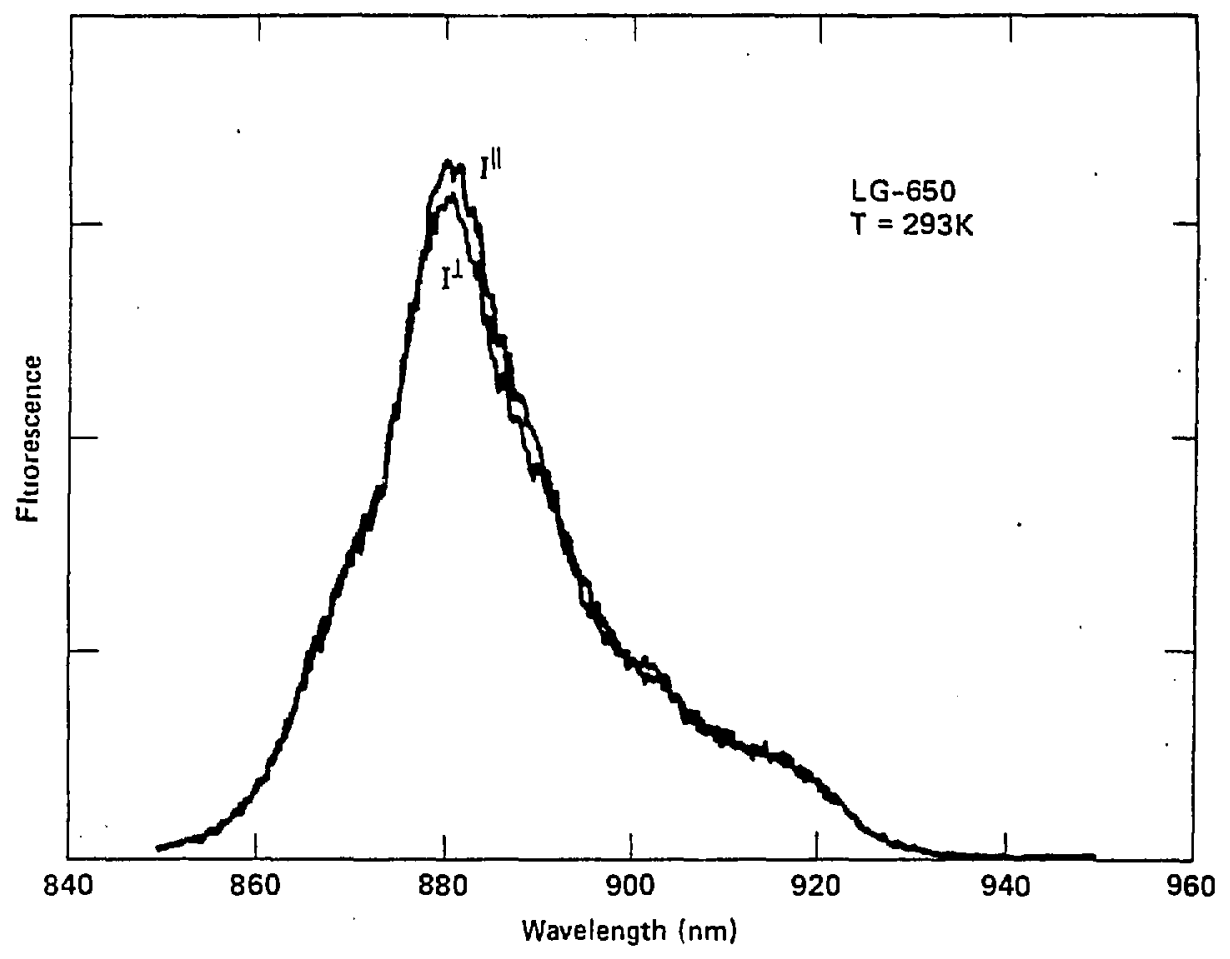

Figure 8: Polarized fluor scence spectra of the $\mathrm{Nd}^{3}+4^{4} \mathrm{~F}_{3 / 2} \rightarrow{ }^{4} \mathrm{Ig}_{\mathrm{g}}$ transition in LG-650 silicate glass. excited by $1.064 \mu \mathrm{m}$ laser light. 
resonant measurement is bolstered by the larger value of $\rho$ measured at $880 \mathrm{~nm}$ compared with that measured at $1.06 \mu \mathrm{m}$ and especially by the fact that $\rho=1$ at wavelengths removed from $880 \mathrm{~nm}$. This latter fact shows that $I^{\perp}$ is not less than I" simply as a result of some unknown characteristic of the apparatus. These results, discussed in the next section, are expected as a result of accidental coincidences.

The polarization capability of the beam optics and the polarization purity of the observation train was verified in situ by inputting unpolarized white light (which was then polarized by G2) and scattering it off a mirror in place of the sample, directiy into the spectrometer. Rotating the half-wave plate resulted in periodic oscillation of signal at $1.06 \mu \mathrm{m}$ with minimum count rate equal to the dark count.

The apparatus was taken apart, reassembled (with some component changes), and $p$ remeasured. Results were identical.

Finaliy, resonant and nonresonant results compare with those for other RE ions and similar hosts with more easily measured transitions (see next section).

F. Discussion of Polarized Fluorescence Results

Values of the depolarization ratio, $\rho$, for several RE ions in glass (Ref. 7),.along with those obtained for $\mathrm{Nd}$, are given in Table II and exhibit two notable trends. These trends may be understood within the semiclassical model of assigning dipole oscillators to the different transitions that make up absorption and fluorescence bands. This model is developed more fully for $N d$ in the next chapter. 
Table II. Resonant and nonresonant depolarization ratios for several RE ions and hosts.

\begin{tabular}{|c|c|c|c|c|}
\hline Ion & $\begin{array}{l}\text { Kramers' } \\
\text { Degenerate }\end{array}$ & Host & $\begin{array}{c}\rho \\
\text { Resonant } \\
\end{array}$ & $\begin{array}{c}\rho \\
\text { Nonresonant } \\
\end{array}$ \\
\hline$E u^{3+}$ & No & Phosphate & 0.43 & 0.81 \\
\hline $\mathrm{Tb}^{3+}$ & No & Phosphate & 0.77 & 0.94 \\
\hline $\mathrm{Er}^{3+}$ & Yes & Tellurite & - & 0.92 \\
\hline $\mathrm{Nd}^{3+}$ & Yes & ED..2 & 0.89 & 0.92 \\
\hline $\mathrm{Nd}^{3+}$ & Yes & LG-650 & 0.88 & 0.95 \\
\hline
\end{tabular}

Sources: Ref. 7 (Eu, Tb, Er) and this paper (iid). 
First, the depolarization ratio of a nonresonant transition for a given ion-host combination is always greater than that of a resonant transition. As discussed in Chapter 2, RE ions in glass experience a variety of local environments, resulting in a distribution of energy splittings of Stark states and an inhomogeneous broadening of spectral lines. Although the ions excited by a narrow band laser pulse are a subset of the dopant population that have at least a pair of states separated by a photon energy, there is not necessarily a correlation. between other Stark states of this subset. ${ }^{8}$ In other words, accidental coincidences (Chapter 2) can prevent FLN experiments from being completely site selective. Imagine these laser-selected ions: the orientation of the dipole(s) that represents a nonresonant transition relative to the orientation of the absorbing dipole(s), may vary, presumably about some average orientation. This variation in orientation resuits in a "smearing" of the emitted polarization which is manifested in a larger depolarization ratio. This effect is in addition to that which results from the isotropically oriented distribution of sites assumed in an amorphous host. The fact that the depolarization ratio is less than unity for some portions of a nonresonant fluorescence band indicates some correlation between dipole orientations of different states. For the $\mathrm{Nd}^{4} \mathrm{~F}_{3 / 2} \rightarrow{ }^{4} \mathrm{I}_{9 / 2}$ transition, excited in the center of the ${ }^{4} \mathrm{I}_{\mathrm{I} / 2} \rightarrow{ }^{4} \mathrm{~F}_{3 / 2}$ band, this correlation decreases as one approaches the fluorescence band edges i.e. $\rho-1$ (see Figures 7 and 8). Analagous results dealing with spectral line-narrowing of nonresonant fluorescence have also been observed. See, for example, the ${ }^{4} \mathrm{~F}_{3 / 2} \rightarrow{ }^{4} \mathrm{I}_{9 / 2} \mathrm{FLN}$ spectrum of Brawer and Weber. 8 
The second point to note in Table II is that the depolarization ratio is generally greater for ions with an odd number of electrons, i.e. Kramers' degenerate systems (see Chapter 2). The low symmetry of the host's crystal field completely removes the degeneracy of a $J$ manifold for an even-electron ion but odd-electron ions retain doubly degenerate Stark states. The non-degenerate states are well-described by a single dipole oscillator while Kramers' degenerate states require several non-collinear oscillators. Transitions of a Kramers' system are said to be described by partially anisotropic oscillators as opposed to a non-degenerate system which is described by completely anisotropic osciilators $^{9}$ (i.e. pure dipoles). The fact that an odd-electron ion may relax via several non-collinear dipole transitions also "smears" the polarization effect, increasing $\rho$. Europium exhibits a resonant depolarization ratio ${ }^{7}$ of 0.43 which is close to that expected for a collection of isotropically oriented identical dipoles, $\rho=1 / 3$ (see Chapter 9); our Nd measurements give a much higher ratio $(\rho=0.88)$. Another point to note comes from a careful study of polarized fluorescence of $\mathrm{Eu}^{3+}$ in $\mathrm{glass}$ made by Lebedev and Przhevuskii. 10 They used alumino-silicate glasses with alkaline and alkaline earth modifiers, as well as $\mathrm{La}\left(\mathrm{PO}_{3}\right)_{3}$ glass, and obtained similar values of $\rho$ for all hosts. Similarly, our results for Nd in two silicate glasses agree within experimental error, even though they were chosen for their different modifier composition and radiative properties. This does not definitively establish a host independence of polarization properties, however. 


\section{References}

1. Phototubes with InGaAsP photocathodes were at one time manufactured by Varian (VPM-159) and had relatively high quantum effic:encies at $1.06 \mu \mathrm{m}$. These tubes have been used in resonant $\mathrm{Nd}$ experiments (e.g. C. Brecher, L. A. Riseberg, and M. J. Weber, Phys. Rev. B 18 5799;' Ref. 5 below) but their production was discontinued, mainly as a result of the instability of the photocathode.

2. A valuable reference on polarized emission is $P$. P. Feofilov, The Physical Basis of Polarized Emission, (Consultants Bureau, New York, 1961).

3. Chapter 2 and references therein.

4. W. F. Krupke, IEEE J. Quant. Elect. QE-10, 450 (1974).

5. S. A. Brawer and M. J. Weber, Appl. Phys. Lett, 35, 31 (1979).

6. See e.g. Y. Beers, Theory of Error, (Addison-Wesley, Palo Alto, 1957).

7. V. P. Lebedev, and A. K. Przhevuskii, Sov. Phys. Solid State 19, 1389 (1979).

8. S. A. Brawer and M. J. Weber, J. Non-Crystalline Solids $\underline{38} / \underline{39}$, $9(1980)$.

9. Ref. 2, page 21.

10. V. P. Lebedev and A. K. Przhevuskii, Opt. Spectrosc. (USSR) 48, 513 (1980). 
Chapter 9: MODEL CALCULATIONS

In this chapter a phenomenological model of cross section anișotropy applicable to $\mathrm{Nd}^{3+}$ ions in glass is presented. The results of polarized FLN and amplifier saturation experiments involving media described by this model are calculated. The influence of a simple model of spectral inhomogeneity on amplifier performance is then investigated. Finally, these models are combined to calculate the effects of poiarization and spectral inhomogeneity in short-plilse glass laser amplifiers.

\section{A. Ion Site Mode?}

We assume transitions between the upper and lower laser levels to be purely electric-dipole with all ion sites identical except for orientation. Thus, this model does not consider the spectral inhomogeneity that will be included in Section $F$ or site-dependent differences in line strength. We assume a two-level system in which Stark splitting and variation of homogeneous linewidths are ignored. The degeneracies of the upper and lower levels are denoted by $g_{4}$ and $g_{1}$, respectively.

Consider a single ion site. The site is, because of the disordered nature of glass, assumed to be of low symmetry (e.g. $\left.C_{1}, c_{2 v}\right)$. A local orthogonal coordinate system denoted by $l, m$, and $n$ may be. uniquely assigned to the site. ${ }^{1}$ A polarized cross section is associated with each axis direcicion as shown in Figure 1 . The cross section $\sigma_{i}$ is proportional to the probability of absorption or emission of light with electric-field vector polarized in the ith direction. We assume that one of the cross sections, $\sigma_{n}$, is larger than the other two and denote its 


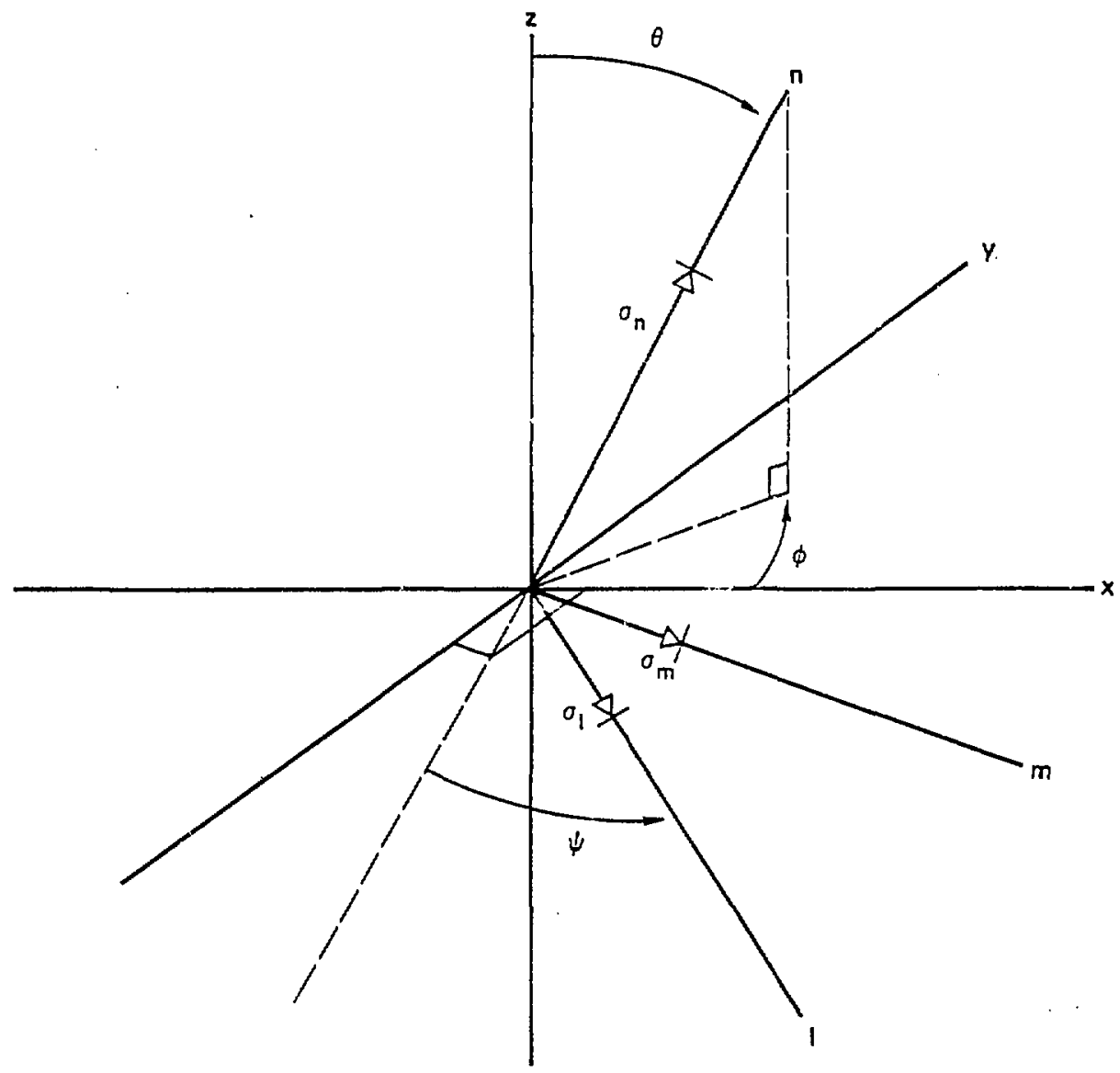

Figure I: Local and laboratory coordinate axes for a model site showing polarized cross sections and Euler's angles. 
value by $\sigma_{p}$. The other two cross sections are assumed equal and are denoted by $s_{s}$ (i.e. $\sigma_{n} \equiv \sigma_{p} ; \sigma_{1}=\sigma_{m} \equiv \sigma_{s}$ ). This assumption simplifies angular integration over all site orientations and permits expression of the inhomogeneity in terms of a single parameter, $\sigma_{s} / \sigma_{p}$. The validity of this assumption vis-a-vis real systems is questionable; there is no a priori reason for equal cross sections in a low-symetry field. The assumption stems iro, a desire to have a simple mathematical model that still allows for site anisotropy and its justification rests ultimately on the ability of the model to predict experimental results.

Cross sections for transitions between either laser level and other levels need not be the same or of the same relative magniture. Thus, experiments designed to probe the laser iransition must be resonant to measure the correct effect of site anisotropy. It is assumed, however, that transitions between pairs of Stark states of the two levels are described by the same ratio of polarized cross sections so that the inhomogeneity is not obscurred by the rapid transfer between degenerate Stark states of a single level.

The sites are assumed isotropically oriented with respect to the laboratory frame, denoted by $X, Y, Z$. The macroscopic response of a system of these sites is obtained by integration over Eulerian angles, $\theta$, $\phi$, and $\psi$ (collectively denoted by $\bar{\theta}$ ).

\section{B. Polarized FLN Calculation}

We now calcusate the fluorescent response of an isotropically oriented collection of these sites that is excited by a beam of light polarized 
$-132-$

along the $Z$ axis and travelling in the $Y$ direction. Fluorescence is observed along the $X$ axis with polarization parallel and perpendicular to that of the exciting beam. Actually, since we are considering electric-dipole transitions, with $z$ as the symmetry axis of the experiment, results are independent of where in the $X-Y$ plane the observation is made.

The probability per unit time of exciting a single ion in a site described by $\bar{\theta}$ with light of frequency $\nu_{L}$ and polarized along $Z$ is given $b y^{2}$

$$
\begin{aligned}
P_{a}^{2}(\bar{\theta}) & =\frac{I^{2}}{h \nu_{L}}\left[\sigma_{p}(\hat{n} \cdot \hat{z})^{2}+\sigma_{s}(\hat{l} \cdot \hat{z})^{2}+\sigma_{s}(\hat{m} \cdot \hat{z})^{2}\right] \\
& =\frac{I^{2}}{h \nu_{L}}\left[\sigma_{p} \cos ^{2} \theta+\sigma_{s} \sin ^{2} \theta\right]
\end{aligned}
$$

The probability per unit time of spontaneous emission with light polarized along $Z$ is given by

$$
P_{e}^{z}(\bar{\theta})=c\left[\sigma_{p}(\hat{n} \cdot \hat{z})^{2}+\sigma_{s}(\hat{\eta} \cdot \hat{z})^{2}+\sigma_{s}(\hat{m} \cdot \hat{z})^{2}\right]=C P_{a}^{z}(\bar{\theta}),
$$

with $C$ a constant.

The probability per unit time of spontaneous emission with light polarized along the $Y$ direction is given by

$$
\begin{aligned}
P_{e}^{y}(\bar{\theta})=c & {\left[\sigma_{p}(\hat{n} \cdot \hat{\gamma})^{2}+\sigma_{s}(\hat{\eta} \cdot \hat{\gamma})^{2}+\sigma_{s}(\hat{m} \cdot \hat{\gamma})^{2}\right] } \\
& =c\left[\sigma_{p} \sin ^{2} \theta \cos ^{2} \phi+\sigma_{s}\left(\sin ^{2} \phi+\cos ^{2} \theta \cos ^{2} \phi\right)\right] .
\end{aligned}
$$


The observed fluorescence intensity is proportional to the probability of absorption times the probability of emission, averaged over all orientations of sites. Hence, the ratio of polarized intensities, $\rho$, is given by

$$
p=\frac{I^{z y}}{I^{z z}}=\frac{\int d \bar{\theta} P_{a}^{z}(\bar{\theta}) P_{e}^{y}(\bar{\theta})}{\int d \bar{\theta} P_{a}^{z}(\bar{\theta}) P_{e}^{z}(\bar{\theta})}
$$

Integration results in a depolarization ratio parametric in the ratio of polarized cross sections, $\sigma_{s} / \sigma_{p}$ :

$$
\rho=\frac{2+16\left(\sigma_{s} / \sigma_{p}\right)+12\left(\sigma_{s} / \sigma_{p}\right)^{2}}{6+8\left(\sigma_{s} / \sigma_{p}\right)+16\left(\sigma_{s} / \sigma_{p}\right)^{2}} \text {. }
$$

Note that when $\sigma_{s}=0$, the system is represented by pure dipoles and $\rho=1 / 3$. When $\sigma_{p}=\sigma_{s}$, the system is represented by isotropic oscillators and $\rho=1$. In future calculations, it is convenient to express the degree of cross section anisotropy by a quantity

$$
\delta \equiv 1-\frac{\sigma_{s}}{\sigma_{p}}
$$

Thus, a system with $\delta=0$ should exhibit no polarization inhomogeneity while one with $\delta=1$, being made up of pure dipole sites, should be the most inhomogeneous.

A plot of the depolarization ratio, $\rho$, as a function of $\delta$ is shown in Figure 2. 


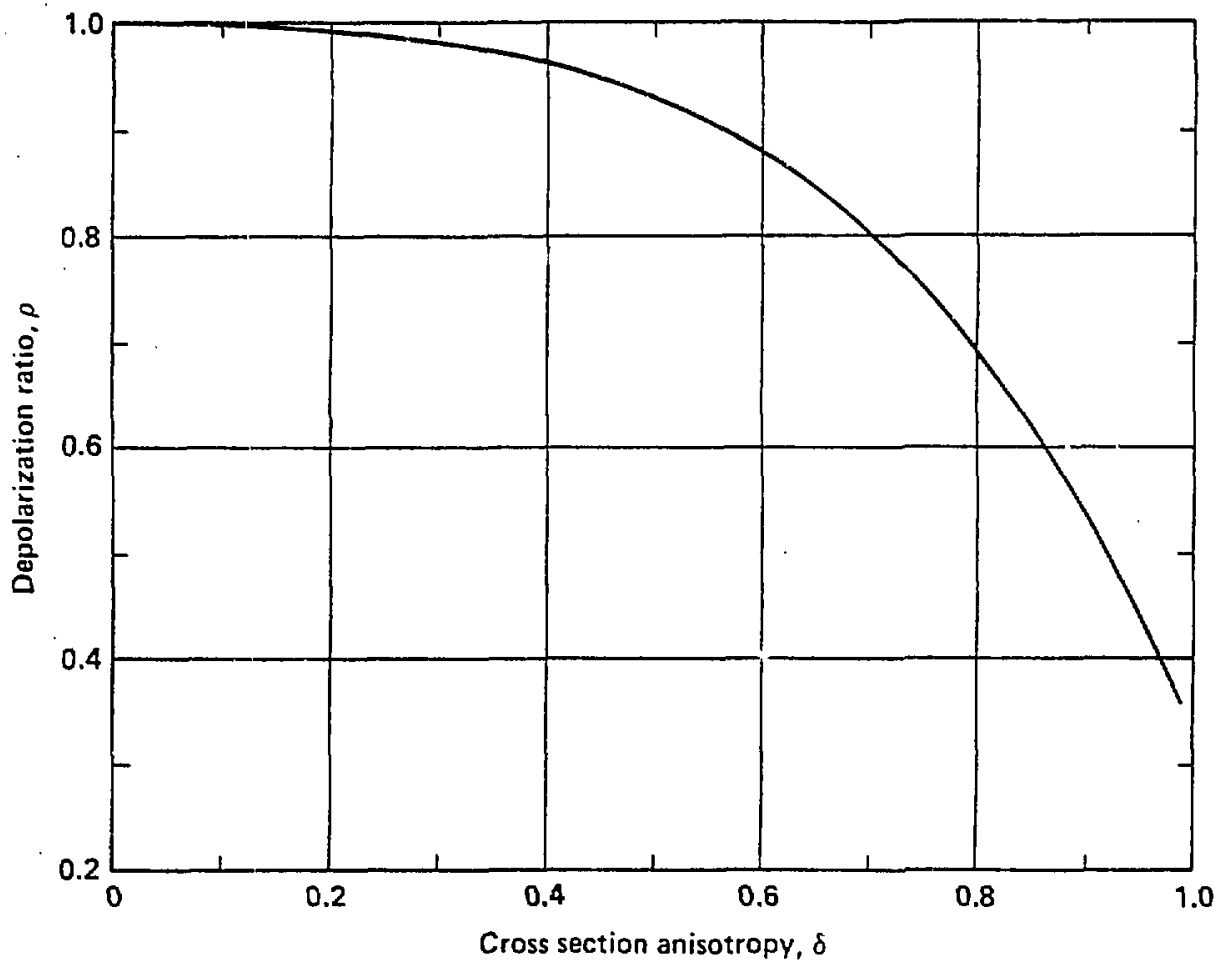

Figure 2: Calculated FLN depolarization ratio, $\rho \equiv I^{\perp} / I^{\prime \prime}$, as a function of the cross section anisotropy, $\delta \equiv 1-\sigma_{s} / \sigma_{p}$, for an isotropically oriented collection of model sites. 


\section{Amplifier Calculations for a General Inhomogeneity}

We now develop the formalism for calculating the amplifier performance of a medium with a general, unspecified inhomogeneity. ${ }^{3}$ In addition to the distribution of cross sections, amplifier calculations require parameters for population rate equations and specification of beam profile and operating conditions. The following treatment assumes parameters appropriate for a Nd:glass amplifier in a fusion laser application. Specifically, the calculation is for a short-pulse laser amplifier in which the spontaneous emission rate and upper level pumping rate are small compared to the stimulated emission rate during extraction. Energy exchange or cross-relaxation between ions is also assumed to be small and are not included in the calculation. This assumption is justified by recent experiments ${ }^{4}$ in which the cross-relaxation rate for Nd in ED-2 laser glass was measured to be $<10^{4} \mathrm{~s}^{-1}$. It is assumed that the lifetime of the lower laser level is long with respect to the duration of the pulse and that this level is initially unpopulated. (There is a great range of published values for this relaxation rate ${ }^{5,6}$ for Nd in glass). The assumption of a lower level "bottle neck" is not crucial to the calculation, however, in that relaxation of this level may be included by an "effective" value for the degeneracy of the lower level (e.g. lower level degeneracy of infinity gives the same results as if the relaxation time constant was zero; see Chapter 10, Section B). We presume propagation along the $X$ axis, a spatially uniform intensity profile in the $Y-Z$ plane, uniform pumping of the medium, and neglect diffraction effects. 
We want the output fluence (energy/area) of the amplifier in terms of the input fluence and an experimentally accessible measure of initial energy storage; for the latter we use the pre-pulse or initial small-signal gain. We also want the gain of a low-intensity probe beam that traverses the amplifier after the large, saturating pulse. The probe beam may differ from the saturating pulse in frequency and/or polarization.

Ions with identical optical properties are assigned to classes labeled by the value of the variable $\xi$. This parameter may represent, for example, the center frequency of the homogeneous line of a class or the orientation of a class with respect to the laboratory coordinate axes. It may be multidimensional to allow for several sources of inhomogeneity. Each class of ion has a stimulated emission cross section $\sigma^{\alpha}(\xi)$ that measures its interaction with a beam of intensity $I^{\alpha}(x, t)$, where $\alpha$ denotes the polarization and frequency characteristics of the beam.

The population rate equations for upper and lower laser levels for a class of ions with parameter from $\xi$ to $\xi+d \xi$ are

$$
\begin{aligned}
& \frac{\partial N_{u}}{\partial t}(x, \xi, t)=-\sigma^{\alpha}(\xi)\left(N_{u}-\frac{g_{u}}{g_{1}} N_{1}\right) \frac{I^{\alpha}(x, t)}{h \nu_{L}} \\
& \frac{\partial N_{1}}{\partial t}(x, \xi, t)=\sigma^{\alpha}(\xi)\left(N_{u}-\frac{g_{u}}{g_{l}} N_{1}\right) \frac{I^{\alpha}(x, t)}{h \nu_{L}}
\end{aligned}
$$

where $N_{j}$ is the number density of ions per unit $\xi$ in the upper $(u)$ and lower (1) laser level and $\nu_{L}$ is the frequency of the stimulating laser light. We assume the bandwidth of the stimulating light to be small with 
respect to bandwidths associated with the ion transition. (When treating spectral inhomogeneity, we implicitly assume that the width of the broadened fluorescence line is small with respect to its peak frequency so that the resonant frequency of an individual ion class may be approximated by $\left.\nu_{L}\right)$. We have neglected spontaneous radiative and nonradiative terms as well as pumping and cross-relaxation terms. These are assumed negligible on the time scale of the saturating pulse. Equations (6) and (7) may be combined into a single equation for the inversion density, $N(x, \xi, t)$, by subtracting $g_{u} / g_{1}$ times Eq. (7) from Eq. (6):

$$
\begin{aligned}
\frac{\partial N}{\partial t}(x, \xi, t) & \equiv \frac{\partial}{\partial t}\left[N_{u}-\frac{g_{u}}{g_{1}} N_{1}\right] \\
& =-\left(1+\frac{g_{u}}{g_{1}}\right) \sigma^{\alpha}(\xi) N(x, \xi, t) \frac{I^{\alpha}(x, t)}{h \nu} .
\end{aligned}
$$

This equation may be solved in terms of the integrated intensity at $x$ up to time t:

$$
N=N(x, \xi,-\infty) \exp \left[\frac{-1}{\Gamma(\xi)} \int_{-\infty}^{t} I^{\alpha}\left(x, t^{\prime}\right) d t^{\prime}\right]
$$

with

$$
\Gamma(\xi)=\frac{h \nu_{L}}{\left(1+\frac{g_{u}}{g_{1}}\right) \sigma^{\alpha}(\xi)}
$$


The initial condition on the inversion density may be put in a more useful form. The lower laser level is initially unpopulated so that $N(x, \xi,-\infty)=N_{u}(x, \xi,-\infty)$. Also, the assumption of uniform pumping iniplies that this quantity is independent of $x$ and that all ion classes have an equal chance of being excited. Hence,

$$
N(x, \xi,-\infty)=N_{T} P(\xi)
$$

where $N_{T}$ is the total population density in the upper laser level, independent of class, and $P(\xi)$ is the normalized distribution function giving the probability that an ion belongs to class $\xi$. Note that $h \nu_{L} N_{T}$ is the energy density stored in the laser medium before arrival of the saturating pulse.

The radiation transport equation that governs the growth of beam intensity, neglecting diffraction and refraction effects, is

$$
\frac{n}{c} \frac{\partial I^{\alpha}}{\partial t}+\frac{\partial I^{\alpha}}{\partial x}=\int d ; \sigma^{\alpha}(\xi) N(x, \xi, t) I^{\alpha}(x, t) .
$$

Substituting Eq. (9) into Eq. (12), using Eq. (11), and integrating over all time yields

$$
\begin{aligned}
& \frac{n}{c} \int_{-\infty}^{\infty} d t \frac{\partial I^{\alpha}}{\partial t}+\int_{-\infty}^{\infty} d t \frac{\partial I^{\alpha}}{\partial x} \\
& =N_{T} \int d \xi P(\xi) \sigma^{\alpha}(\xi) \int_{-\infty}^{\infty} d t I^{\alpha}(x, t) \exp \left[\left(\frac{-1}{\Gamma(\xi)}\right) \int_{-\infty}^{t} I^{\alpha}\left(x, t^{\prime}\right) d t^{\prime}\right] .
\end{aligned}
$$


The first term on the left of Eq. (13) is zero as a result of the finite duration of the input pulse. To obtain an equation in terms of fluence, define $\gamma$ such that

$$
\gamma(x, t)=\int_{-\infty}^{t} I^{\alpha}\left(x, t^{\prime}\right) d t^{\prime} .
$$

This quantity is seen to be the fluence at $x$ up to time $t$. Also define $\gamma(x, \infty)=\Gamma(x)$ and note that $\gamma(x,-\infty)=0$. Then, since $d \gamma=1\left(x, t^{\prime}\right) d t^{\prime}$, Eq. (13) becomes

$$
\frac{d}{d x} \int_{0}^{\Gamma} d \gamma=N_{T} \int d \xi P(\xi) \sigma^{\alpha}(\xi) \int_{0}^{\Gamma} d \gamma \exp \left[-\frac{\gamma}{\Gamma(\xi)}\right]
$$

or, performing the integration over $\gamma$,

$$
\frac{d}{d x}=N_{T} \int d \xi P(\xi) \sigma^{\alpha}(\xi) \Gamma(\xi)\left(1-e^{-\Gamma / \Gamma(\xi)}\right)
$$

Recalling the definition of $\Gamma(\xi)$ from Eq. (10), this becomes

$$
\frac{d \Gamma}{d x}=\frac{h \nu_{L} N_{T}}{\left(1+\frac{g_{u}}{g_{l}}\right)} . \int d \xi P(\xi)\left[1-e^{-\Gamma / \Gamma(\xi)}\right]
$$

In this form, the equation for fluence gain is seen to be an expression of conservation of energy. The exponential is the probability that an ion of class $\xi$ is still in the excited state after experiencing a fluence $\Gamma$. The quantity in brackets is, therefore, the probability that an ion 
has made the trinsition and contributed its energy to the beam. This probability is averaged over all classes and is multiplied by the total energy density and a degeneracy factor that accounts for the unavoidable absorption of laser light by a fraction of ions that have previously been de-excited.

The initial total population density of the upper level is a difficult quantity to directly assess in an amplifier saturation experiment. However, we may obtain an expression for it in terms of the small-signal gain of the system before arrival of the saturating pulse. In the small-signal regime, the exponential in $\Gamma$ may be expanded and Eq. (17) becomes, using Eq. (10),

$$
\begin{aligned}
\frac{d \Gamma}{d x} & \simeq \mathrm{N}_{T} \Gamma \int \mathrm{d} \xi P(\xi) \sigma^{\alpha}(\xi) \\
& =\left(\bar{\sigma} \mathrm{N}_{T}\right) \Gamma
\end{aligned}
$$

where the fact that $P(\xi)$ is the probability distribution of ions among classes has been used to obtain the initial average, or "small-signal", cross section of the system. The quantity in parentheses is seen to be the pre-pulse small-signal gain coefficient that governs the usual unsaturated exponential growth of signal with distance in an amplifier. With this denoted by $g_{0}$, Eq. (17) may be expressed as

$$
\frac{d \Gamma}{d x}=\frac{h \nu_{L}}{\left(1+\frac{g_{u}}{g_{1}}\right)} \frac{g_{0}}{\sigma}\left[1-\int d \xi P(\xi) e^{-\Gamma / \Gamma(\xi)}\right]
$$


where we have used the fact that $P(\xi)$ is normalized to unity.

This equation may be integrated from $x=0$ to $x=L$, where $L$ is the length of the amplifier, to obtain the output fluence, $\Gamma(L)$ for a given input fiuence, $\Gamma(0)$.

The gain for a weak $\mathrm{CW}$ probe beam after the saturating pulse may be obtained from the following equation:

$$
\frac{d I^{P}}{d x}=\int d \xi \sigma^{P}(\xi) N(x, \xi, \infty) I^{P}(x)
$$

where $\sigma^{P}(\xi)$ is the cross section presented by an ion in class $\xi$ to a beam with polarization and frequency ?abelled by the superscript p. A "weak" probe is one that does not significantly change the inversion density as a result of its amplification. So?ving this equation gives an expression that may be integrated to obtain $G^{P} \equiv I^{P}(x) / I^{P}(0)$ :

$$
\frac{d\left(\ln G^{p}\right)}{d x}=\int d \xi o^{P}(\xi) N(x, \xi, \infty)
$$

or, using Eqs. (9), (11), and (18) for the inversion density after the saturating pulse, $N(x, \xi, \infty)$,

$$
\frac{d\left(\ln G^{P}\right)}{d x}=g_{0} \int d \xi \frac{\sigma^{P}(\xi)}{\bar{\sigma}} P(\xi) e^{-\Gamma / \Gamma(\xi)} .
$$

The quantity in $G^{P}$ may be recognized as the local gain coefficient for material at position $x$. 
In anticipation of the need to numerically integrate Eqs. (19) and (22) for cross sections and distributions of interest, we may put them in dimensionless form by defining a small-signal saturation parameter, $\Gamma_{5}$ :

$$
\Gamma_{s}=\frac{h \nu_{L}}{\left(1+\frac{g u}{g}\right)} \bar{\sigma}
$$

Then, if $\phi \equiv \Gamma / \Gamma_{\mathrm{s}}$ is the normalized fluence, and $u=\frac{x}{L}$ denotes normalized distance along the amplifier (with $0 \leq u \leq 1$ ), Eq. (19) becomes

$$
\frac{d \phi}{d u}=g_{0} L\left[1-\int d \xi P(\xi) e^{-\phi \frac{\sigma^{\alpha}(\xi)}{\sigma}}\right]
$$

and Eq. (22) becomes

$$
\frac{d\left(\ln G^{P}\right)}{d u}=g_{0} L \int d \xi P(\xi) \frac{\sigma^{p}(\xi)}{\sigma} e^{-\phi \frac{\sigma^{\alpha}(\xi)}{\sigma}} .
$$

D. Amplifier Calculation for Polarization Inhomogeneity

The results of the preceeding section are applied to the isotropically oriented collection of anisotropic sites described in Section $A$ and used to model the polarized FLN experiment in Section $B$. The class parameter $\xi$ becomes the Euler angles for a site, $\bar{\theta}$. To proceed, we need expressions for $\sigma^{\alpha}(\xi), P(\xi)$, and $\bar{\sigma}$. From Section $A$, for light polarized along the $Z$ axis, an expression for the cross section is 


$$
\begin{aligned}
\sigma(\bar{\theta}) & =\sigma_{p} \cos ^{2} \theta+\sigma_{s} \sin ^{2} \theta \\
& =\sigma_{p}\left(1-\delta \sin ^{2} \theta\right)
\end{aligned}
$$

with

$$
\delta=1-\frac{\sigma_{S}}{\sigma_{p}}
$$

The cross section for $Z$ polarization is seen to be independent of $\phi$ and $\psi$ and these variables may be dropped from the following equations. The probability distribution function, $P(\theta)$, is known because of the assumed isotropy of orientations:

$$
P(\theta)=A \sin \theta
$$

where $\sin \theta$ is included from solid-angle considerations and the normalization constant, $A$ can be determined since we require

$$
\int_{0}^{\pi} P(\theta) d \theta=1
$$

Performing this integration yields $A=1 / 2$. The small-signal effective cross section may be obtained using Eqs. (26) and (27) by integrating over all $\theta$ :

$$
\bar{\sigma}=\frac{\sigma_{\mathrm{p}}}{2} \int_{0}^{\pi} d \theta \sin \theta\left(1-\delta \sin ^{2} \theta\right)
$$


or, letting $v=\cos \theta$,

$$
\begin{aligned}
\bar{\sigma} & =\frac{\sigma_{p}}{2} \int_{-1}^{i} d v\left[(1-\delta)+\delta v^{2}\right] \\
& =\sigma_{p}[1-(2 / 3) \delta]
\end{aligned}
$$

Using these expressions in the general equation for fluence gain, Eq. (24) becomes

$$
\frac{d \phi}{d u}=g_{0} L\left[1-\frac{1}{2} \int_{0}^{\pi} d \theta \sin \theta e^{-\phi \frac{\left(1-\delta \sin ^{2} \theta\right)}{1-(2 / 3) \delta}}\right] .
$$

Letting $v=a \cos \theta$, in becomes

$$
\frac{d \phi}{d u}=g_{0} L\left[1-\frac{1}{2 a} e^{-\phi \frac{(1-\delta)}{1-(2 / 3) \delta}} \int_{-a}^{a} d v e^{-v^{2}}\right]
$$

where

$$
a \equiv\left[\frac{\phi \delta}{1-(2 / 3) \delta}\right]^{\frac{1}{2}}
$$

The integral can be recognized as related to the error function ${ }^{7}$ defined by

$$
\operatorname{Erf}(a)=\frac{2}{\sqrt{\pi}} \int_{0}^{a} d v e^{-v^{2}}
$$


Thus, Eq. (32) becomes

$$
\frac{d \phi}{d u}=g_{0} L\left[1-e^{-\frac{\phi(1-\delta)}{1-(2 / 3) \delta}} \frac{\sqrt{\pi}}{2 a} \operatorname{Erf}(a)\right] .
$$

It is usefur to examine this equation in the extremes of cross rection ratio: i) $\delta=0$, implying isotropic sites and $i i) \delta=1$, implying pure dipole sites.

i) $\delta=0$ : As $\delta$ approaches zero the argument of the error function in Eq. (35) aiso approaches zero. An expansion of the error function for small argument yields the limit for the terms involving a in Eq. (35):

$$
\lim _{a \rightarrow 0} \frac{\sqrt{\pi}}{2 a} \operatorname{Erf}(a)=\lim _{a \rightarrow 0} \frac{1}{a}\left(a-\frac{a^{3}}{3}+\frac{a^{5}}{10}-\ldots\right)=1
$$

Thus Eq. (35) becomes

$$
\frac{d \phi}{d u}=g_{0} L\left(1-e^{-\phi}\right)
$$

This can be analytically integrated along the amplifier to obtain

$$
\phi(L)=\ln \left[e^{g_{0} L}\left(e^{\phi(0)}-1\right)+1\right],
$$

which is normalized fluence expression of the Frantz and Nodvik result for saturation in a homogeneous amplifier. Our small-signal saturation parameter, $\Gamma_{\mathrm{s}}$, reduces to Frantz and Nodvik's scaling parameter, $\Gamma_{\mathrm{FN}}$, (often called "the saturation fluence") in the limit of no inhomogeneity. 
ii) $\delta=1$ : In this case Eq. (35) becomes

$$
\frac{d \phi}{d u}=g_{0} L\left[1-\frac{\sqrt{\pi}}{2} \frac{1}{\sqrt{3 \phi}} \operatorname{Erf}(\sqrt{3 \phi})\right] \text {. }
$$

In the limit of small $\phi$, using the expansion Eq. (36), this becomes

$$
\begin{aligned}
\frac{d \phi}{d u} & =g_{0} L\left[1-\left(1-\frac{1}{3}(\sqrt{3 \phi})^{2}+\sigma\left(\phi^{2}\right)\right)\right] \\
& =g_{0} L
\end{aligned}
$$

This result implies exnonential growth of the signal along the length of the amplifier and demonstrates that the small-signal behavior of an inhomogeneous system is indistinguishable from that of a homogeneous amp 1 ifier.

The gein of a weak probe with polarization parallel to the preceeding saturating pulse is, from Eq. (25),

$$
\frac{d\left(\ln G^{\prime \prime}\right)}{d u}=g_{0} L \int_{0}^{\pi} d \theta \frac{\sin \theta}{2} \frac{\left(1-\delta \sin ^{2} \theta\right)}{1-(2 / 3) \delta} e^{-\phi \frac{\left(1-\delta \sin ^{2} \theta\right)}{1-(2 / 3) \delta}} .
$$

Transforming variables as above, this becomes

$$
\frac{d\left(7 n G^{\prime \prime}\right)}{d u}=g_{0} L \frac{e^{-\frac{\phi}{1-(2 / 3) \delta}} e^{a^{2}}}{1-(2 / 3) \delta}\left[\frac{1-\delta}{a} \int_{0}^{a} e^{-v^{2} d v+\frac{\delta}{a^{3}}} \int_{0}^{a} v^{2} e^{-v^{2}} d v\right] \text {. }
$$


The second integral may be done by parts to yield an error function, so that Eq. (42) becomes

$$
\begin{aligned}
& \frac{d\left(\ln G^{\prime \prime}\right)}{d u}=\frac{g_{0} L}{1-(2 / 3) \delta}\left[(1-\delta) \frac{\sqrt{\pi}}{2 a} \operatorname{Erf}(a) e^{-\frac{\phi(1-\delta)}{1-(2 / 3) \delta}}\right. \\
& \left.-\frac{\delta}{2 a^{2}} e^{-\frac{\phi}{1-(2 / 3) \delta}}+\frac{\delta}{2 a^{2}} \frac{\sqrt{\pi}}{2 a} \operatorname{Erf}(a) e^{-\frac{\phi(1-\delta)}{1-(2 / 3) \delta}}\right]
\end{aligned}
$$

In the limit as $\delta=0$ this can be seen to reduce to the homogeneous result for the post-pulse gain coefficient i.e.

$$
g(u)=g_{0} e^{-\phi}
$$

To calculate the gain of a probe with polarization perpendicular to the saturating pulse, we need an expression for the cross section for light polarized along $Y$. From Section $B$, this is

$$
\sigma(\theta, \phi)=\sigma_{p} \sin ^{2} \theta \cos ^{2} \phi+\sigma_{s}\left(\sin ^{2} \phi+\cos ^{2} \theta \cos ^{2} \phi\right) .
$$

Since the cross section is now a function of $\phi$, the distribution function must also include this variable so that

$$
\int_{0}^{2 \pi} d \phi \int_{0}^{\pi} d \theta P(\theta, \phi)=1
$$


The sites are assumed uniformiy distributed along an arc represented by d $\phi$ so that

$$
P(\theta, \phi)=\frac{1}{4 \pi} \sin \theta
$$

where the coefficient is determined by normalization requirements. With these functions, Eq. (25) becomes

$$
\begin{gathered}
\frac{d\left(\ln G^{+}\right)}{d u}=g_{0} L \int_{0}^{2 \pi} d \phi \int_{0}^{\pi} d \theta \frac{\sin \theta}{4 \pi}\left[\sin ^{2} \theta \cos ^{2} \phi+\frac{\sigma_{s}}{\sigma_{p}}\left(\sin ^{2} \phi+\cos ^{2} \theta \cos ^{2} \phi\right)\right] x \\
\frac{1}{1-(2 / 3) \delta} \exp \left[-\frac{\phi\left(1-\delta \sin ^{2} \theta\right)}{1-(2 / 3) \delta}\right] .
\end{gathered}
$$

Performing the integration over $\phi$ and transforming variables yields

$$
\frac{d\left(i n G^{L}\right)}{d u}=\frac{g_{0} L}{2} \frac{e^{-\frac{\phi}{1-(2 / 3) \delta}} e^{a^{2}}}{1-(2 / 3) \delta}\left[(2-\delta) \int_{0}^{a} e^{-v^{2}} d v-\delta \int_{0}^{a} v^{2} e^{-v^{2}} d v\right]^{n}
$$

where the fact that $1+\sigma_{\mathrm{s}} / \sigma_{\mathrm{p}}=1+(1-\delta)$ has been used. Evaluating the integrals over $v$ gives

$$
\begin{aligned}
\frac{d\left(\ln G^{+}\right)}{d u} & =\frac{g_{0} L}{1-(2 / 3) \delta}\left[\left(1-\frac{\delta}{2}\right) \frac{\sqrt{\pi}}{2 a} \operatorname{Erf}(a) e^{-\frac{\phi(1-\delta)}{1-(2 / 3) \delta}}\right. \\
& \left.+\frac{\delta}{4 a^{2}} e^{-\frac{\phi}{1-(2 / 3) \delta}}-\frac{\delta}{4 a^{2}} \frac{\sqrt{\pi}}{2 a} \operatorname{Erf}(a) e^{-\frac{\phi(1-\delta)}{1-(2 / 3) \delta}}\right] .
\end{aligned}
$$


In the homogeneous limit $(\delta=0)$, this expression also reduces to Eq. (44). It may be shown that, for small $\Phi$, expressions for the gain coefficients of both polarizations reduce to $g_{0}$, regardless of $\delta$; the polarization dependence of the post-pulse gain is apparent only when saturation takes place.

The first order differential equations representing fluence growth, Eq. (35), and the growth of polarized small-signal gain, Eqs. (43) and (49), were numerically integrated using a second-order Runge-Kutta method. ${ }^{9}$ Programs, written in FORTRAN for operation on a POP $11 / 40$ minicomputer, are included in Appendix B. The error function was approximated by a polynominal expansion ${ }^{10}$ which was found to be accurate to six significant figures within its domain of validity.

The growth of fluence with distance for conditions in which mild and severe saturation of the amplifier occur is shown in Figures 3 and 4 , for several values of the parameter $\delta$. The deviation from the small-signal exponential growth with distance due to saturation can be seen in Figure 3. The growth shown in Figure 4 is essentially linear and exhibits the usual asymptotic gain behavior of high input fluences. Note when comparing Figures 3 and 4 that the fractional spread of output fluences for the extremes of inhomogeneity $(\delta=0,1)$ remains about the same as the input fluence increases. The inclusion of the input fluence in the total output fluence masks, however, that the fluence gain of a $\delta=1$ system is $78 \%$ of that of a $\delta=0$ system when $\phi(0)=0.25$ but is only $72 \%$ when $\phi(0)=1$. 


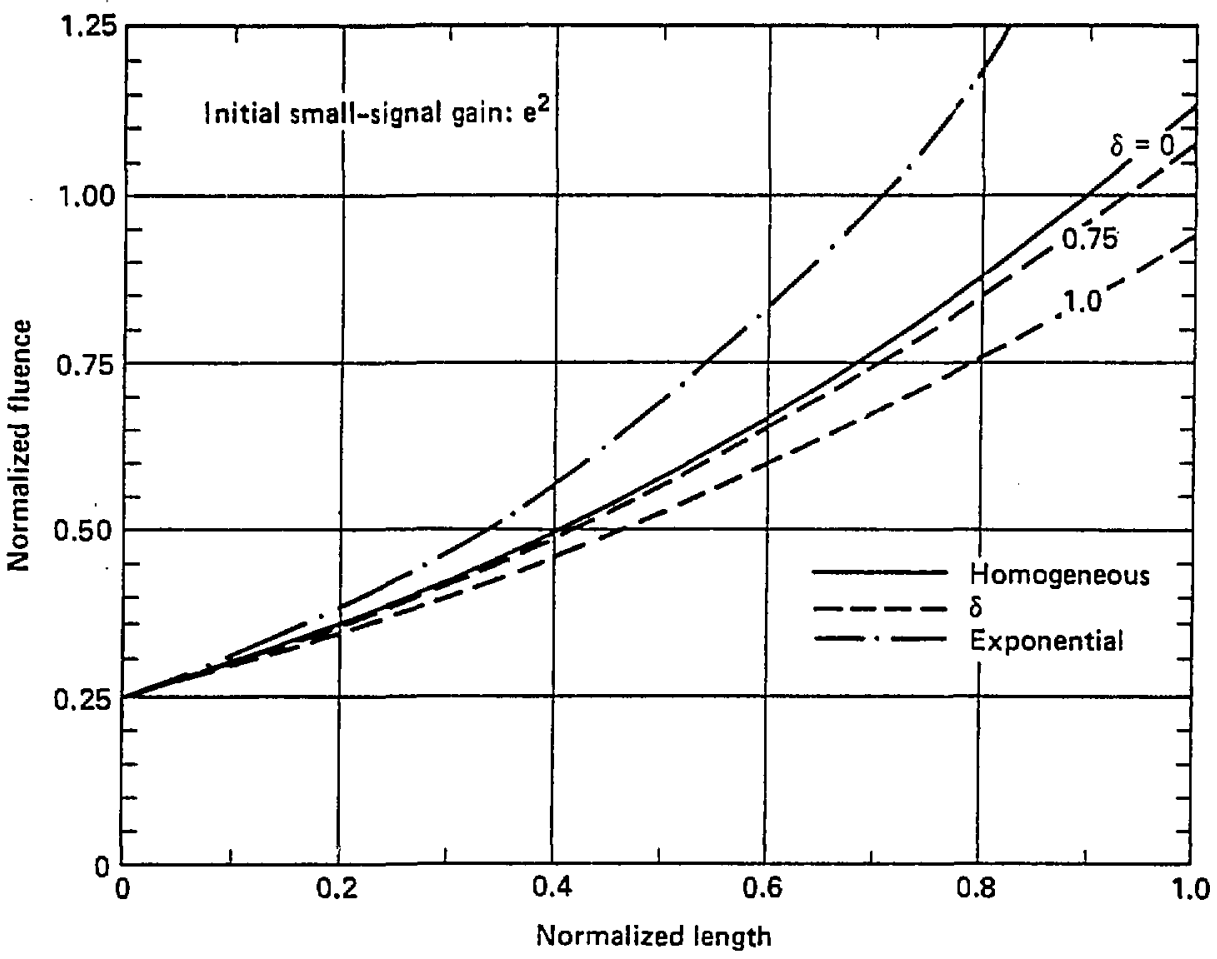

Figure 3: Growth of fluence with length along orientationally inhomogeneous mode 1 amplifiers with several values of cross section anisotropy, $\delta$. The initial small-signal gain of the amplifier is $e^{2}$ and input fluence is 0.25 in normalized units. 


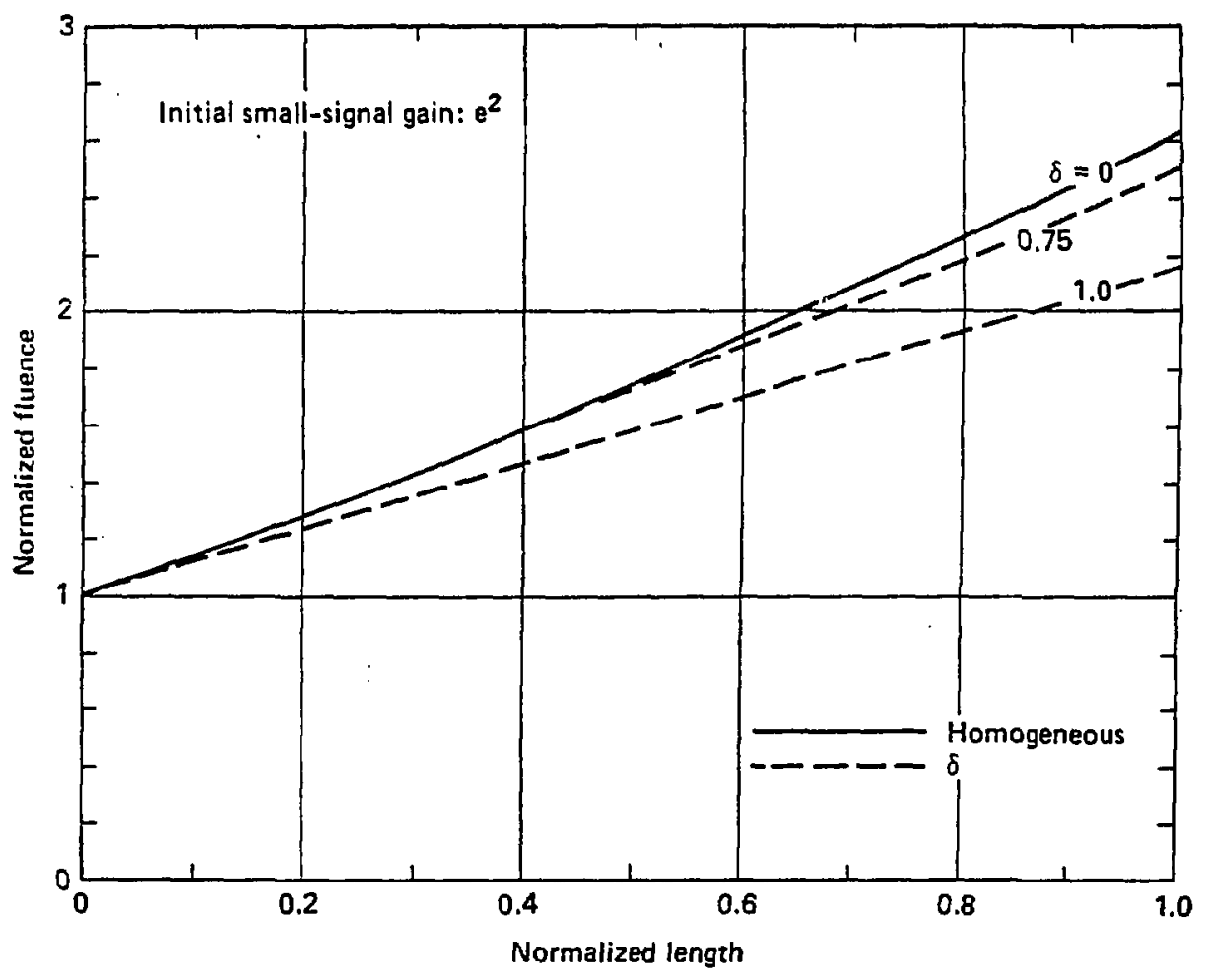

Figure 4: Growth of fluence with length along orientationally inhomogeneous model amplifiers with several values of cross section anisotropy, $\delta$. The initial small-signal gain of the amplifier is $\mathrm{e}^{2}$ and input fluence is 1.0 in normalized units. 
The decrease in the local polarized gain coefficients after the saturating pulse'is shown in Figure 5 for an amplifier with the same parameters as that of Figure ?. These quantities are a measure of the remaining inversion density at a given position and decrease with distance as a result of increasing fluence along the amplifier. One may imagine measuring these gain coefficients with probe beams along the $y$ direction polarized along $z(\operatorname{lnG})$ and along $X\left(1 n G^{2}\right)$. This follows from the symmetry implied by the assumption of electric-dipole transitions.

The growth of intensity of polarized small-signal probes that are collinear with the saturating pulse are shown in Figure 6 after passage of the pulse. The ratio of parallel to perpendicular post-pulse gains is seen to decrease with increasing inhomogeneity. This ratio is not only a function of $\delta$ but also the degree of saturation that takes place as is demonstrated in Figure 7 for several values of 8 with amplifiers pumped so they have an initial small-signal gain of $e^{2}$. The ratio initially decreases with increasing fluence as those sites with large cross section axis parallel to the polarization of the pulse are preferentially de-excited. As $\boldsymbol{\phi}(0)$ gets still larger, however, other orientations also get de-excited and, since parallel sites are essentially already de-populated, the ratio increases.

Figure 8 shows the output fluence as a function of input fluence, parametric in $\delta$, for this same initial small-signal gain. Behavior of these different $\delta$ amplifiers is identical in the small-signal regime but diverges with increasing saturation. 


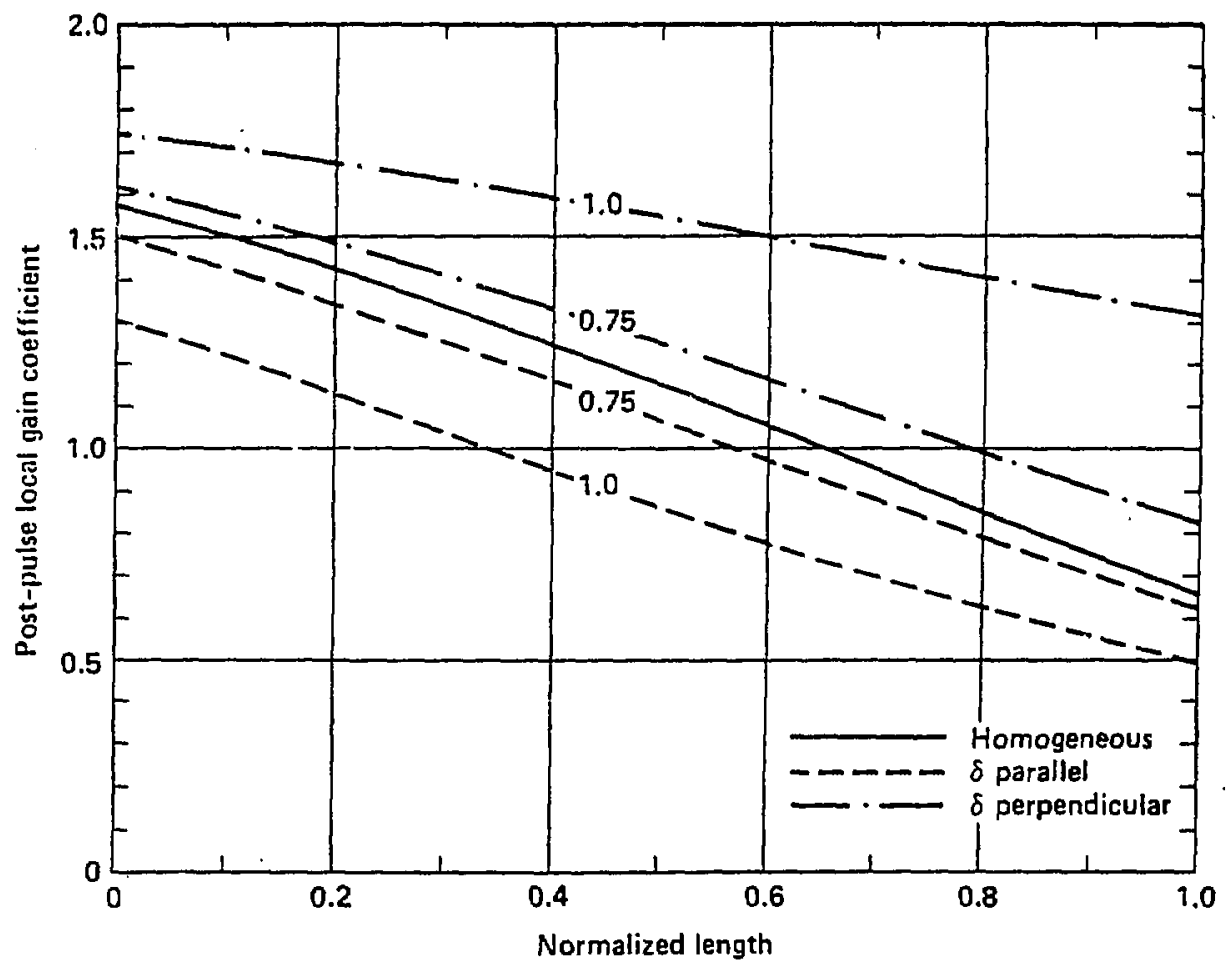

Figure 5: Local polarized gain coefficients along orientationally inhomogeneous amplifiers after passage of a polarized saturating pulse. Initial smal1-signal gain coefficient $=2.0$; normalized input fluence $=0.25$. 


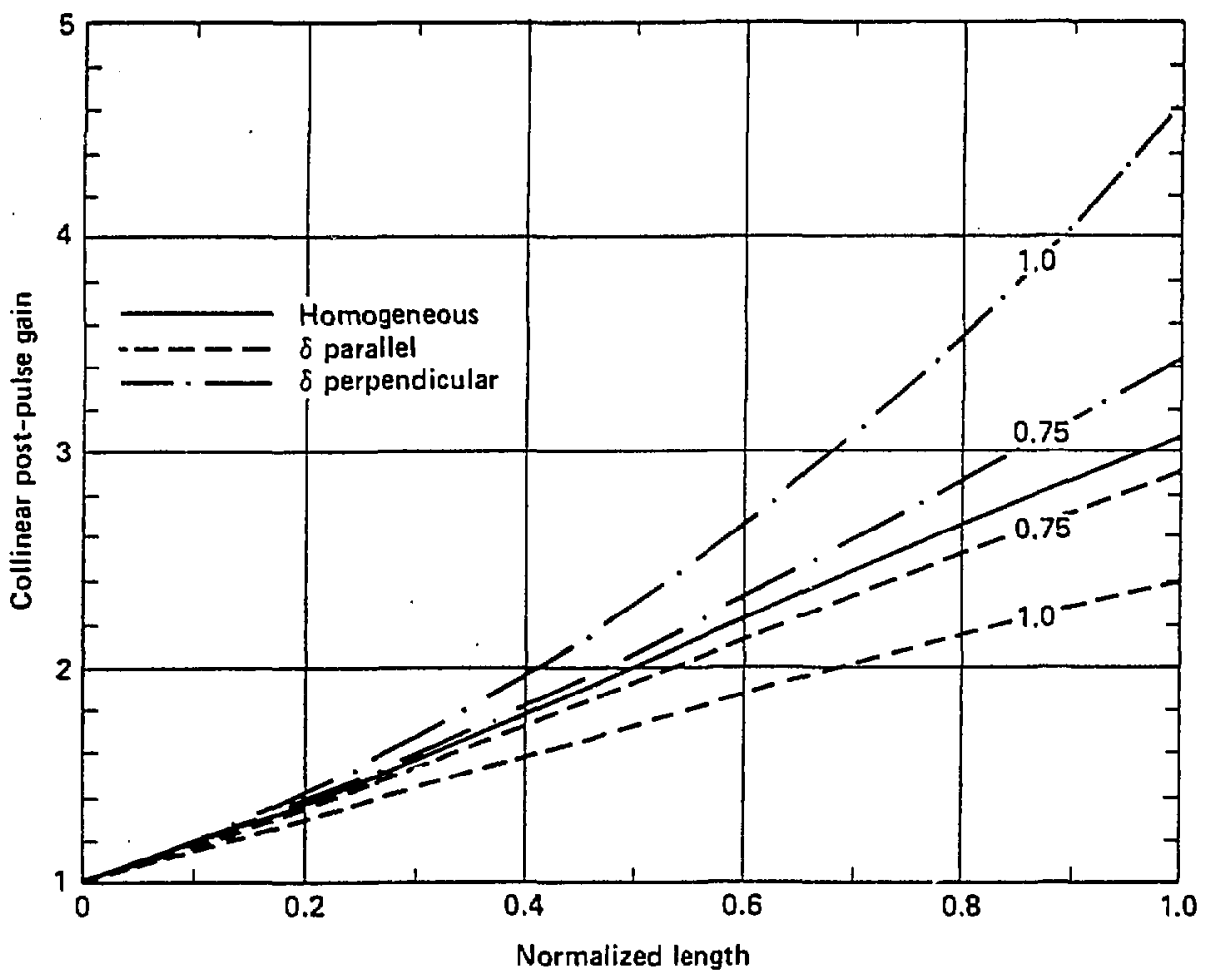

Figure 6: Growth of polarized probe intensity when collinear with the saturatir.g pulse for orientationally inhomogeneous amplifiers. Initial small-signal gain $=e^{2}$. Normalized input fluence $=0.25$. 


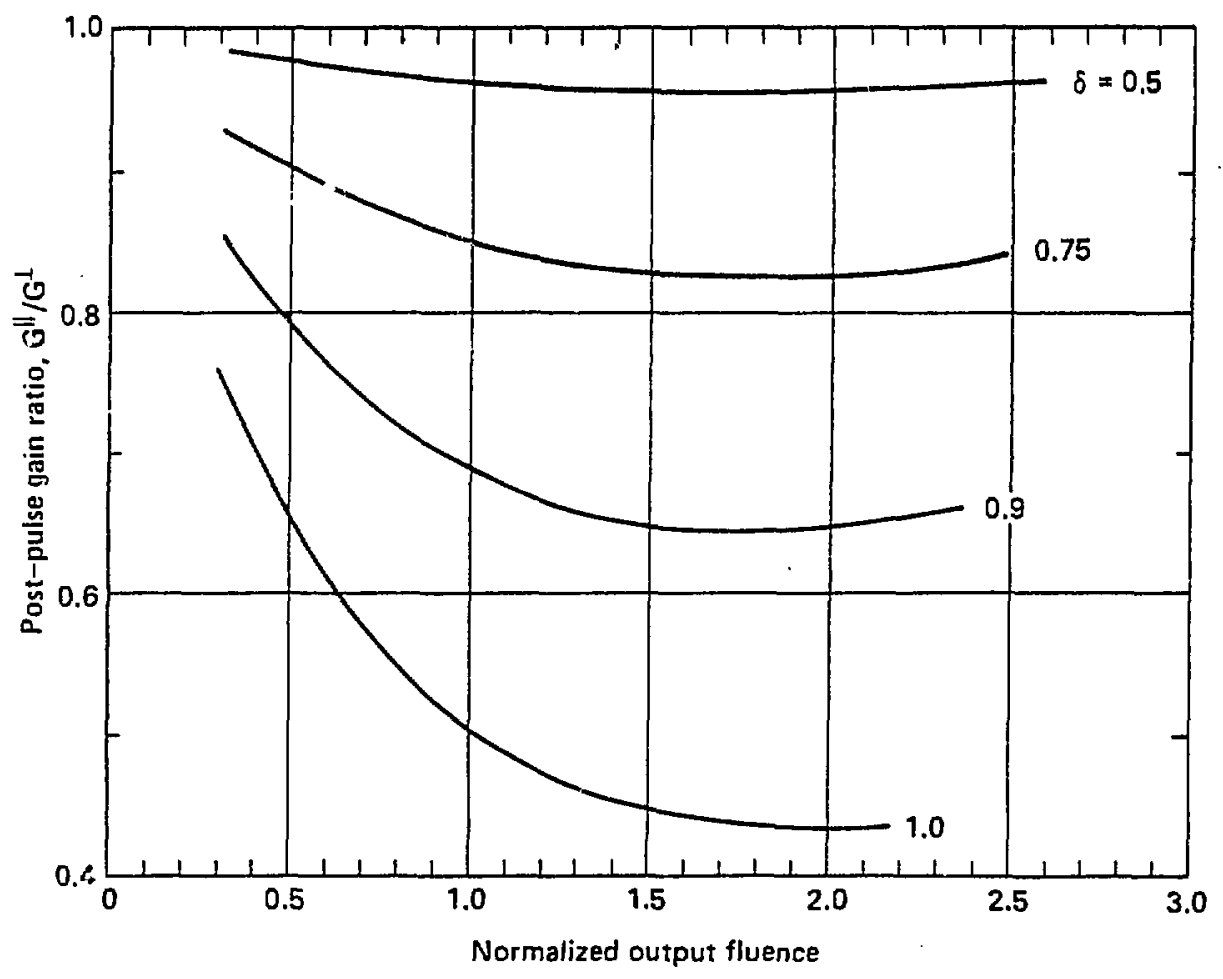

Figure 7: Post-pulse polarized gain ratio, $G^{\prime \prime} / G^{\perp}$, as a function of output fluence of the saturating pulse for orientationaliy inhomogeneous amplifiers. These are gains measured by probes propagating collinear with the saturating pulse. Initial.small-signal gain $=\mathrm{e}^{2}$. 


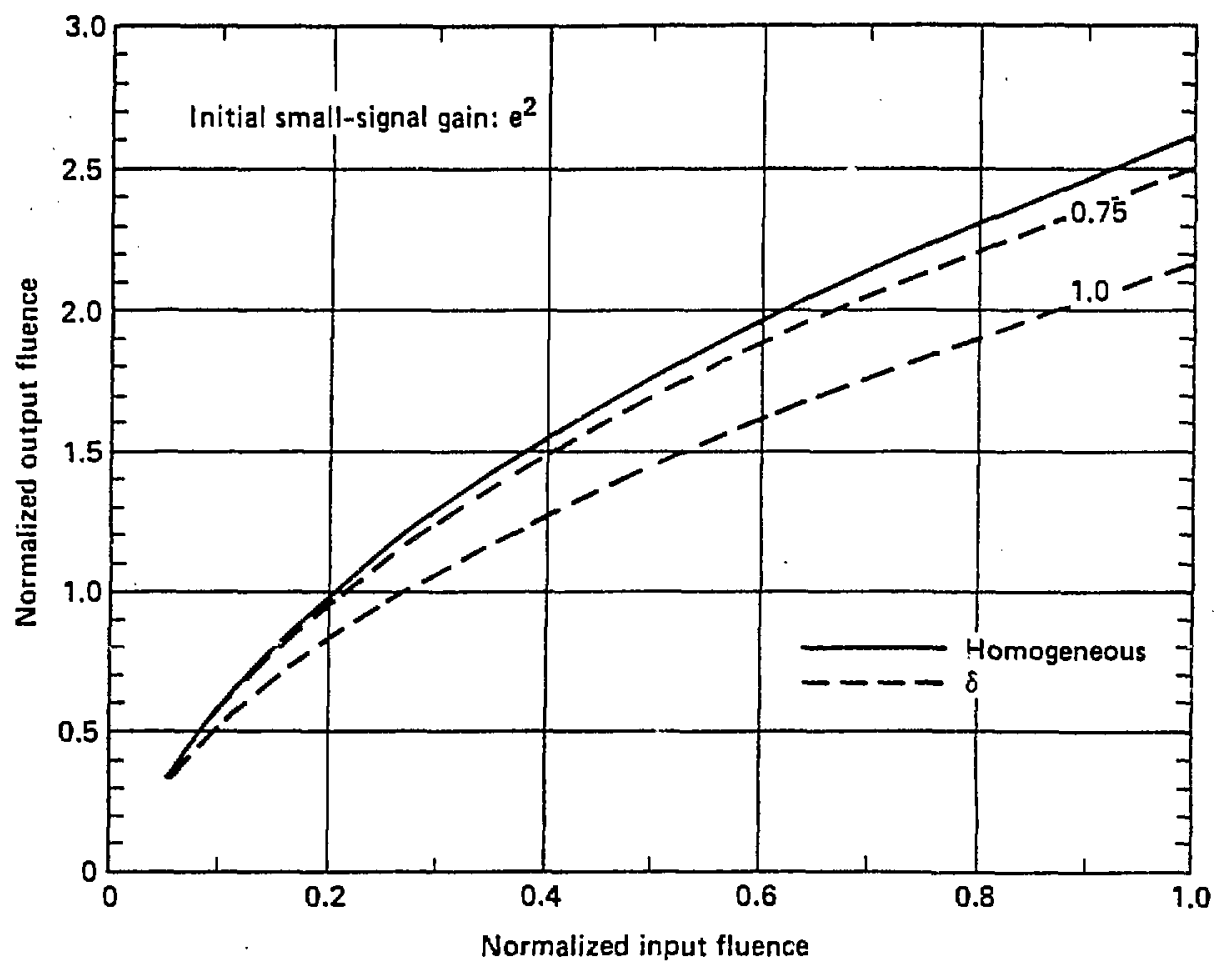

Figure 8: Normalized output fluence vs. input fluence for orientationally inhomogeneous amplifiers. 
A quantity of interest in amplifier design and one that depends on operating parameters is the extraction efficiency, defined as the ratio of energy gain of the amplified beam to the extractable energy initially stored in the system. The extractable energy for a two-level system with no drain from the lower laser level is equal to the stored energy divided by the degeneracy factor. In the case of uniform plane geometry, a useful expression of extraction efficiency, $\eta$, is

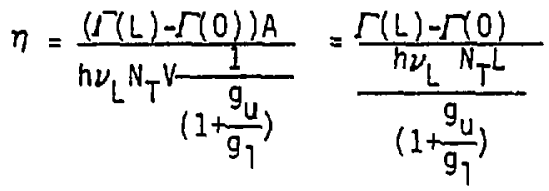

where $V=A^{\circ} L$ is the volume of the amplifier. Since, from $\varepsilon q$. (18) $N_{T}=g_{0} / \bar{\sigma}$, this quantity may be expressed in terms of our normalized fluence as

$$
\eta=\frac{\phi(L)-\phi(0)}{g_{0} L}
$$

The extraction efficiency as a function of input fluence for an amplifier with an initial small-signal gain of $e^{2}$ is shown in Figure 9, parametric in $\delta$.

All curves approach an extraction efficiency of unity with increasing input fluence. This indicates that the inhomogeneity does not limit the extractable energy. However, to get the same fraction of stored energy out of an amplifier, one must operate a more inhomogeneous system at higher fluence levels, an important consideration for designs in which the 1 imiting design factor is component damage. 


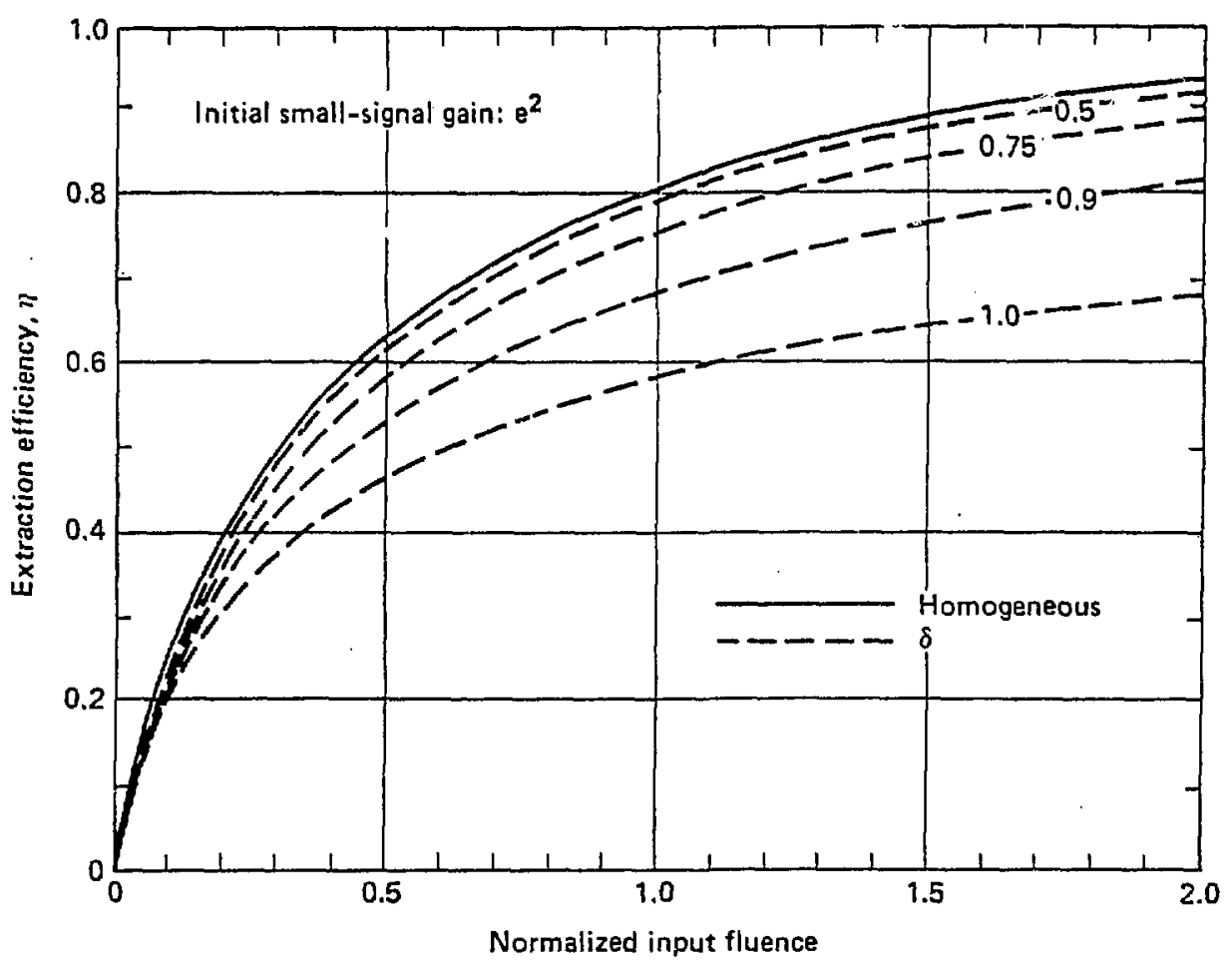

Figure 9: Extraction efficiency $\eta$, vs. normalized input fluence for orientationally inhomogeneous amplifiers. 


\section{E. Amplifier Calculation foi Spectral Inhomogeneity}

The generai results of Section $C$ for short-pulse amplification are applied to a specific model of spectral inhomogeneity. The model treats the broad fluorescence line of the laser transition as a superposition of Lorentzian lines of equal homogeneous 1 inewidth whose center frequencies are distributed about the observed fluorescence peak with Gaussian probability. This model accurately describes Doppler broadening in gases $^{11}$ and has been used to model CW glass amplifiers. ${ }^{12}$ The class parameter becomes $\nu_{\xi}$, the peak frequency of an ion's Lorentzian line. If we assume that the laser is operated at the fluorescence peak frequency, $\nu_{0}$, an expression for an ion's cross section is

$$
\sigma\left(\nu_{\xi}\right)=\sigma_{0}\left\{1+\left[\frac{2\left(\nu_{\xi}-\nu_{0}\right)}{\Delta \nu_{h}}\right]^{2}\right\}
$$

where $\sigma_{0}$ is the Lorentzian line-center cross section and $\Delta \nu_{h}$ is the full width at half-maximum (FWHM) of an individual ion's line. The normalized Gaussian distribution function is

$$
P\left(\nu_{\xi}\right)=\frac{2}{\Delta \nu_{i h}}\left(\frac{\ln 2}{\pi}\right)^{\frac{1}{2}} \exp \left[-\left(2 \sqrt{\ln 2} \frac{\nu_{\xi}-\nu_{0}}{\Delta \nu_{i h}}\right)^{2}\right]
$$

where $\Delta \nu_{i h}$ is the FWHM of the inhomogeneous spectral distribution. By making the change of variables

$$
v=\frac{2 \sqrt{1 \pi 2}}{\Delta \nu_{i h}}\left(\nu_{\xi}-\nu_{0}\right)
$$




$$
\bar{\sigma}=\frac{\sigma_{0}}{\sqrt{\pi}} \int_{-\infty}^{\infty} d v \frac{e^{-v^{2}}}{1+\left(\frac{v}{\beta}\right)^{2}}
$$

where

$$
\beta=\sqrt{\ln 2} \frac{\Delta \nu_{h}}{\Delta \nu_{i h}}
$$

and the assumption that $\nu_{0} \gg \Delta \nu_{i h}$ has been used to extend the lower 7 imit of integration to $-\infty$. The integral in Eq. (55) may be recognized as related to the Voigt function, ${ }^{13}$ defined as

$$
H(\beta, \zeta)=\frac{1}{\beta \pi} \int_{-\infty}^{\infty} d y \frac{e^{-y^{2}}}{1-\left(\frac{y-\zeta}{\beta}\right)^{2}}
$$

Thus,

$$
\bar{c}=\sigma_{0} \sqrt{\pi} \beta H(\beta, 0)
$$

Equation (24) for fluence growth then becomes, using Eq. (54),

$$
\frac{d \phi}{d u}=g_{0} L\left\{1 \frac{2}{\sqrt{\pi}} \int_{0}^{\infty} d v \exp \left[-v^{2}-\frac{\phi}{\left(1+\left(\frac{v}{\beta}\right)^{2}\right) \sqrt{\pi} \beta H(\beta, 0)}\right]\right\}
$$

Examining this equation in the rimit that $\beta \rightarrow \infty$ (i.e. $\Delta \nu_{i h} \rightarrow 0$ so that the medium is spectraliy homogeneous), we see that 


$$
\begin{aligned}
\frac{d \phi}{d u} & =g_{0} L\left[1-e^{-\phi} \frac{2}{\sqrt{\pi}} \int_{0}^{\infty} e^{-v^{2}} d v\right] \\
& =g_{0} L\left[1-e^{-\phi}\right]
\end{aligned}
$$

where we have used the fact that

$$
\lim _{\beta \rightarrow \infty} \sqrt{\pi} \beta H(\beta, 0)=1
$$

as can be seen from Eq. (56). Equation (59) may be integrated to obtain the Frantz-Nodvik result of Eq. (38).

The code for numerical integration and solution of the differential equation for fiuence gain, Eq. (58), is included in Appendix B. The Voigt function, which may be expressed as the real part of the complementary error function of a complex variable, ${ }^{13}$ was approximated using a polynominal expansion. 10

The extraction efficiency as a function of input fluence for an amplifier with an initial small-signal gain of $e^{2}$ is shown in Figure 10 with $\Delta \nu_{h} / \Delta \nu_{i h}$ as a parameter. As in the case of the polarization inhomogeneity moder, the inhomogeneity does not limit the extractable energy, but requires increasing input fluence to obtain the same efficiency with systems of increasing inhomogeneity.

Unlike the polarization model, this model has no limit to the degree of inhomogeneity. As $\Delta \nu_{h} / \Delta \nu_{i h} \rightarrow 0$, the curve of extraction efficiency drops toward zero. 


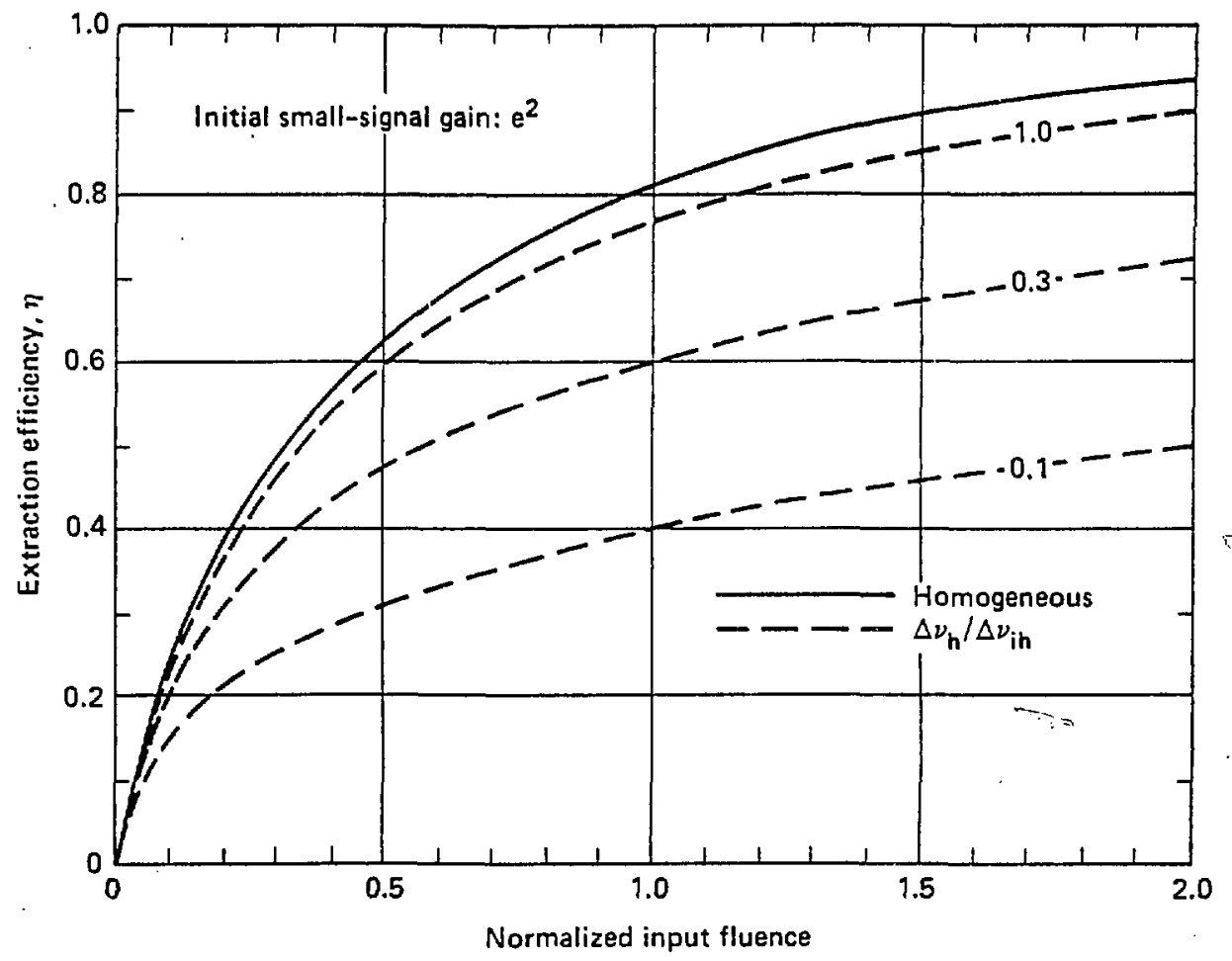

Figure 10: Extraction efficiency, $\eta$, vs. normalized input fluence for spectrally inhomogeneous amplifiers. Initial small-signal gain $=e^{2}$. 


\section{F. Spectral and Polarization Inhomogeneity}

To describe a system in which both spectral and polarization hole burning occur, the models of the preceeding two sections may be combined. A reasonable way of doing this is to let each ion, with a site anisotropy described by $\delta$, be described spectrally as a Lorentzian line with a Gaussian probability of distribution about the fluorescence peak frequency. The class parameter becomes multi-dimensional $\left(\xi=\bar{\theta}, \nu_{\xi}\right)$. The cross section, distribution function, and effective small-signal cross section are simply products of the quantities found in Sections D and $E$. Integrated results are not separable, however, because the exponential in the equation for fluence gain, Eq. (24), now contains the product of the individual cross sections.

Formulas for the fluorescence growth and the growth of polarized post-pulse small-signal gain are straight-forward but unaesthetic. Although the integral over $\theta$ may be expressed in terms of an error furction, explicit integration of this variable by trapezoidal rule was chosen due to the limited domain of validity of polynominal approximations of this function. The double integration calculation was checked with each of the individual calculations in the appropriate limits. The code necessary for the calculations is included in Appendix $B$.

A comparison of output fluence as a function of input fluence for several values of $\delta$ and $\Delta \nu_{h} / \Delta \nu_{i h}$ is made in Figure 11 and extraction efficiencies for these systems are shown in Figure 12. The influence of spectral hole burning on the minimum value of post-pulse polarized gain ratio is slight. The output fluence at which this minimum occurs decreases, however, with increasing spectral inbomogeneity. 


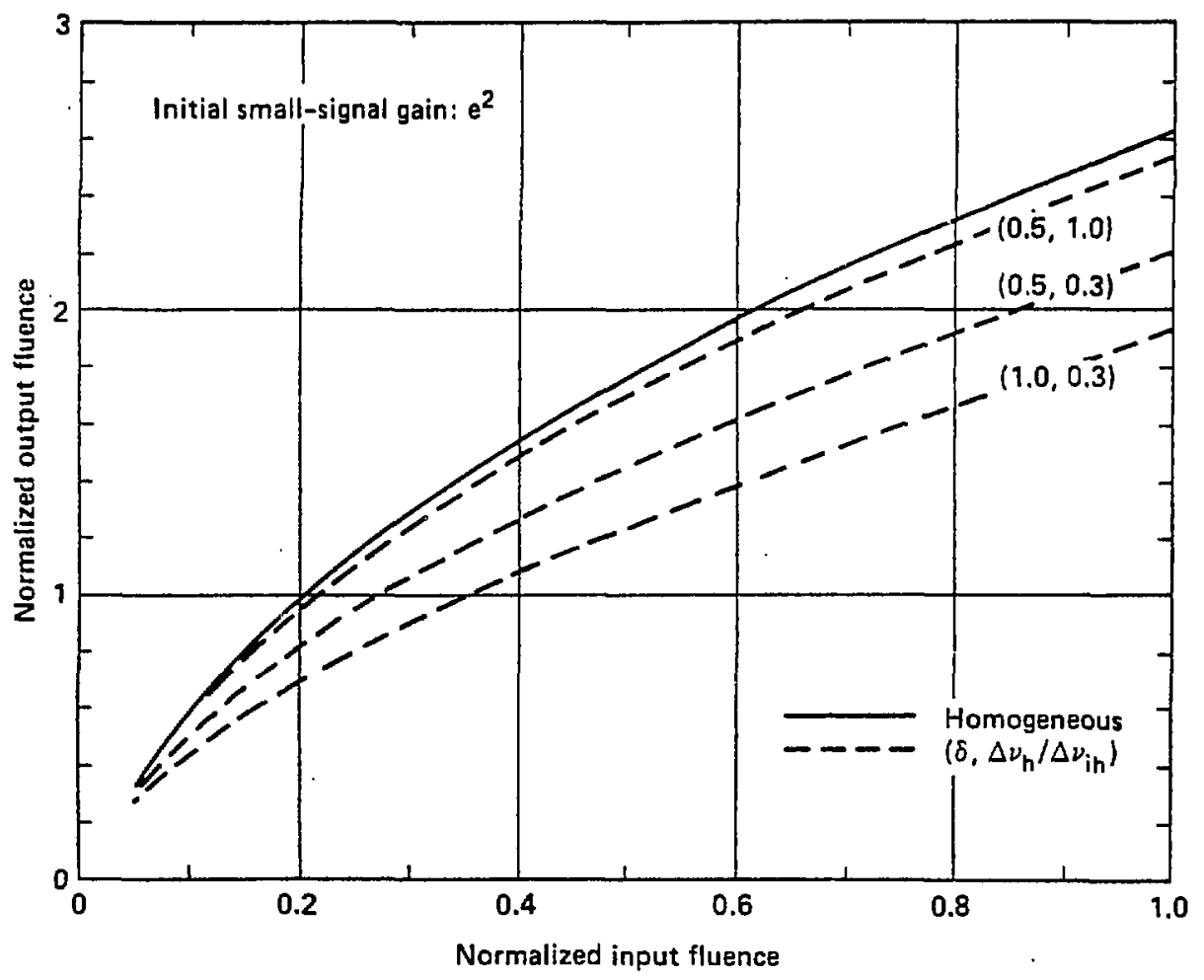

Figure 11: Normalized fluence output vs. fluence input for spectrally and or ientationally inhomogeneous model amplifiers. Initial small-signal gain $=e^{2}$. 


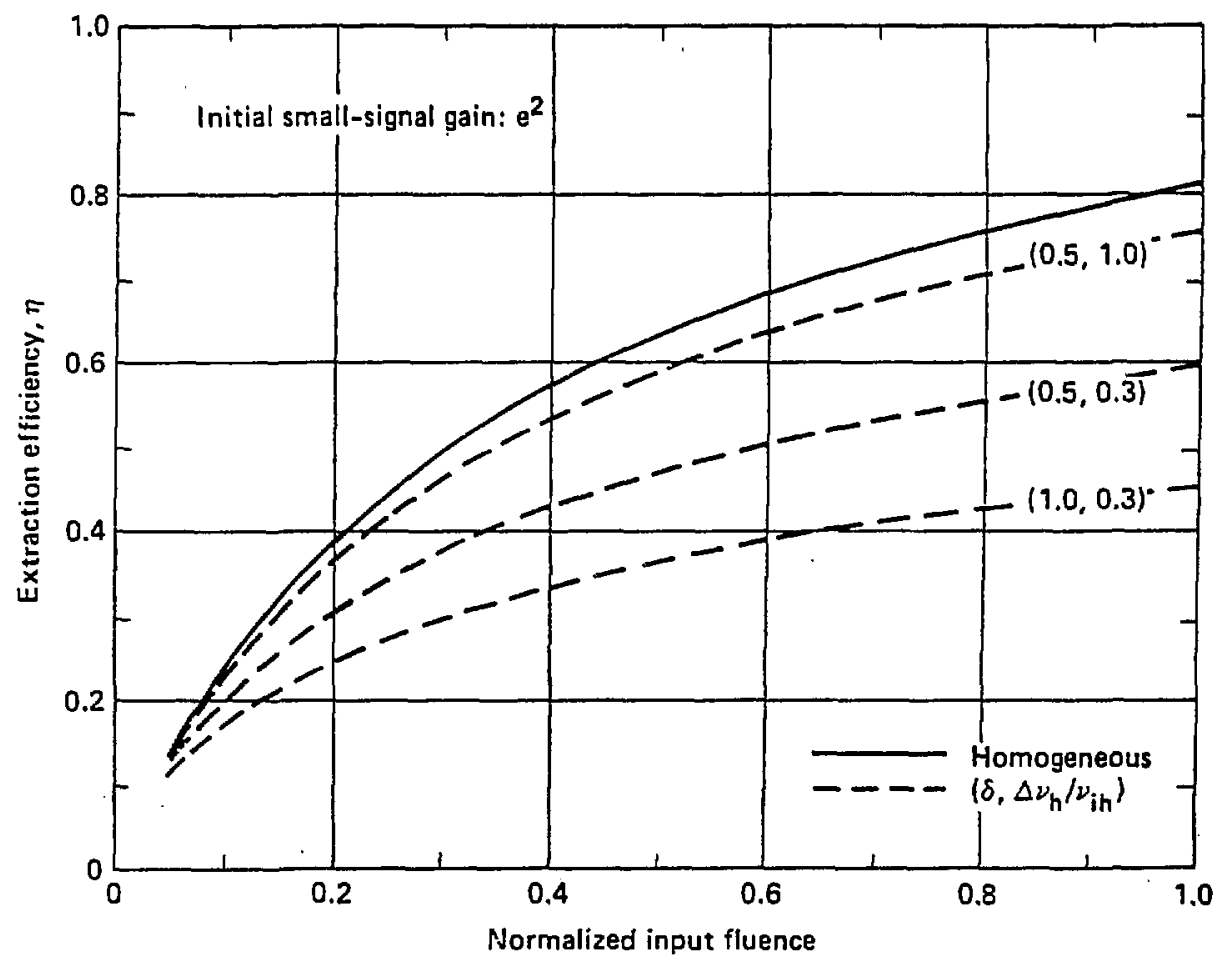

Figure 12: Extraction efficiency, $\eta$, vs, normalized input fluence for spectrally and orientationally inhomogeneous amplifiers. Initial small-signal gain $=e^{2}$. 


\section{References}

1. S. A. Brawer and M. J. Weber, J. Non-Crystalline Solids, $\underline{38 / 39} 9$ (1980).

2. See e.g. E. U. Condon and H. Odinshaw, Handbook of Physics (McGravi-Hill, New York, 1958) p. 2-5 for a discussion of Euler's angles and the projection of axes onto the laboratory frame.

3. This formalism is, essentially, a synthes is of one presented by $R$. Haas during the U. C. Davis AS265 Laser Physics Course (1981) and one he developed while first exploring the effects of polarization hole burning.

4. S. A. Brawer and M. J. Weber, Appl. Phys. Lett. 35, 31 (1979).

5. J. M. McMahon and J. L. Emmett, Record of 11 th Symposium on Electron, Ion, and Laser Beam Technology, Boulder 1971 (San Francisco Press 1971).

6. Yu. P. Rudnitskii, R. V. Smirnov, and V. M. Chernyak, Sov. J. Quant. Elect. $\underline{6}, 1107$ (1976).

7. M. Abramowitz and I. A. Stegun, Handbook of Mathematical Functions (National Bureau of Standards 1970) p. 297.

8. Ibid., p. 297 , formula 7.1.5.

9. See e.g. R. W. Hornbeck, Numerical Methods, (Quantum Publishers, New York 1975), p. 194.

10. M. Abramowitz and I. A. Stegun, Handbook of Mathematical Functions (National Bureau of Standards 1970) p. 299, formula 7.1.26. 
$-167-$

11. E. I. Gordon, A. D. White, and J. D. Rigden, Proc. of the Symposium on Optical Masers, 1963 (Polytechnic Press, New York) p. 309.

12. K. N. Seeder, IEEE Transactions ED-2, 63 (1965).

13. D. W. Posener, Aust. J. Phys. 12, p. 184 (1959). 
Chapter 10: COMPARISON OF MODEL CALCULATIONS WITH SATURATION EXPERIMENTS

Results of computer calculations based on the model of spectral and orientational inhomogeneity developed in the previous chapter are now compared with the amplifier gain experiments of Martin and Milam ${ }^{1-4}$ and Yarema and Milam. 5 First, their experimental data is presented and discussed. Next, the parameters and scaling quantities necessary to compute gain and saturation behavior are determined. Not all quantities needed for these calculations are known for Nd:glass media; estimates of unknown quantities are made using data from FLN and small-signal measurements for the most widely studied silicate glass, ED-2. Experimental and computational results of output vs. input fluence and polarized post-pulse small-signal gain are then compared using these "best guess" parameters. The model is then applied to a cormercial phosphate glass, Q-88 (Kigre, Inc.), using parameters extrapolated from those for ED-2 via established empirical relationships.

\section{A. Experimental Saturation Data}

Martin, Milam, and Yarema have made extensive saturation measurements of $\mathrm{Nd}$-doped laser media using a variety of experimental configurations, glass types, and initial small-signal gains. In these measurements, the sample was the active medium in an operating laser rod amplifier. An inversion was created by flashlamp pumping and a temporally and spatially well-characterized linearly-polarized pulse was amplified by the device. Input and output energies for each shot were determined calorimetricaliy and the time-resolved polarization-dependent gain of the amplifier was measured concurrently using a $\mathrm{CW}$ probe detected with photodiodes. For details of these experiments, the reader is referred to the ir papers. ${ }^{1-5}$ 
Input and output fluence data from Refs. 1 and 2 for ED-2 are given in Table I on a shot-to-shot basis. For all shots listed, the amplifier had an initial small-signal gain of $6.2 \pm 0.2$. The uncertainty represents the spread of gains accepted for comparison with a model gain of 6.2 and not an estimate of error involved in the experimental determination of the gain $(=1 \%)$. The calorimetrically measured fluences were believed to be accurate to within $2 \%$. Therefore, when comparing experimental and computational results, data points should be thought of as having uncertainty from two sources: measurement imprecision and the slightly different initial gain conditions associated with variations in flashlamp pumping. Assuming for simplicity that these uncertainties combine approximately linearly, data have an effective error bar of $\simeq 5 \%$. Data for saturating pulse lengths of 1,10 , and $50 \mathrm{~ns}$ are included in the table. Fluence data for Q-88, with an initial small-signal gain of 7.0 and saturating pulse length of $1 \mathrm{~ns}$, are given in Table II (Ref. 5). A real amplifier rod or disk has passive transmission losses (due to inclusions, striations, bubbles, etc.) which decrease the amplification of a signal along its length by scattering the beam. Although samples were of high quality, there were observable passive losses $(3.8 \%$ for the ED-2 rod and $1.0 \%$ for the Q-88 rod). The model of Chapter 9 does not include these losses which must be accounted for when calculations and experimental data are compared. This may be accomplished by using values of the Frantz and Nodvik saturation parameter, $\Gamma_{F N}$, calculated on a shot-to-shot basis by Martin and Milam. ${ }^{2,5}$ They treated the medium as if it were homogeneous but with passive losses and determined $\Gamma_{F N}$, a 
Table I. ED-2 output vs. input fluence data.

Laser wavelength: $1.064 \mu \mathrm{m}$; initial small-signal gain: $6.2 \pm 0.2$;

linear polarization.

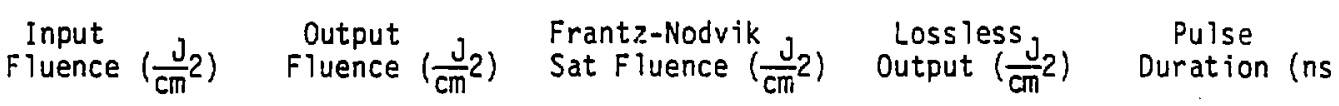

$\begin{array}{llllr}0.077 & 0.426 & 2.19 & 0.442 & 1 \\ 0.096 & 0.541 & 4.45 & 0.562 & 1 \\ 0.187 & 0.989 & 3.30 & 1.025 & 10 \\ 0.249 & 1.26 & 3.37 & 1.31 & 1 \\ 0.486 & 2.3 & 4.24 & 2.38 & 50 \\ 0.652 & 2.79 & 3.49 & 2.88 & 10 \\ 0.943 & 3.82 & 4.22 & 3.95 & 1 \\ 1.14 & 4.48 & 4.54 & 4.63 & 10 \\ 1.32 & 4.99 & 4.55 & 5.15 & 10 \\ 2.62 & 8.21 & 4.09 & 8.45 & 50 \\ 3.4 & 9.82 & 5.35 & 10.12 & 50 \\ 3.59 & 9.95 & 5.22 & 10.25 & 50\end{array}$

Source: W. E. Martin and D. Milam, LLNL UCID Report 18868 (1980). 
Table II. Q-88 cutput vs. input fluence data.

Laser wavelength: $1.053 \mu \mathrm{m}$; initial smal1-signal gain: 7.0;

linear polarization.

Input

Fivence $\left(\mathrm{J} / \mathrm{cm}^{2}\right)$
Output

Fiuence $\left(\mathrm{J} / \mathrm{cm}^{2}\right)$
Loss 7 ess

Output (Jirm)
0.175

0.271

0.366

0.648

0.686

0.917

1.135

1.450

1.502

1.864

1.955

2.302

2. 831
1.00

1.48

1.92

3.06

3.16

3.92

4.57

5.47

5.66

6.48

6.68

7.46

8.52
1.01

1.49

1.94

3.09

$3.1 \mathrm{c}$

3.56

4.62

5.52

5.72

6.54

6.75

7.53

8.61

Source: 5. M. Yarema and D. Milam, "Gain Saturation in phosphate laser glasses", (submitted to IEEE J. Quant. Elect.). 
scaling quantity characteristic of only the amplifying entities of the medium $\left(\Gamma_{\mathrm{s}}\right.$ of Eq. $(9-23)$ reduces to $\Gamma_{\mathrm{FN}}$ in the 1 imit of no inhomogeneity). Their code requires the experimentally determined initial small-signal gain, input fluence, output fluence, and passive loss coefficient. Since the test media are not spectroscopically homogeneous, $\Gamma_{\text {FN }}$ was found to vary with the degree of saturation that occurs during a specific shot; i.e. no single parameter characterizes the saturated gain of the amplifier. However, given their calculated $\Gamma_{F N}$, the initial gain, and the input fluence for a single shot, one may obtain a value for the output fluence by using the Frantz and Nodvik equation (Chapter 9, Eq. 38) which, to first order, would be that measured if passive losses were absent. This value is called the "lossless output" in Table I and differs from the measured output by a factor on the order of the passive loss $0:$ the ED-2 rod. For Q-88 data, the "lossless output" is simply the measured output fluence increased by the $1 \%$ passive loss factor.

The data for the polarized post-pulse gain ratio ${ }^{2}$ as a function of fluence is less complete and is shown in Figure 1 for several glasses in addition to ED-2 and Q-88. The initial small-signal gains of the individual data points are not specified but are assumed to be near the average value for concurrent fluence measurements, i.e. 6.2 for ED-2 and 12.0 for Q-88. (The gain ratio was not measured for the Q-88 fluence Jata of Table II where the amplifier had ar initial gain of 7.0 ; this fluence data was chosen over the data with gain of 12.0 because of its wider range of input and output fluences). The precision of the rati. data may be gauged by the large scatier of data points for a single 


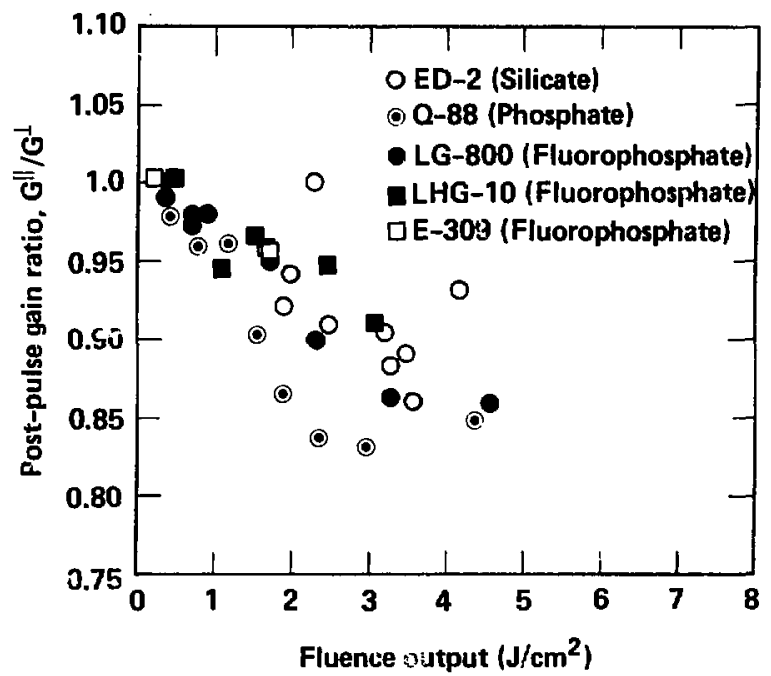

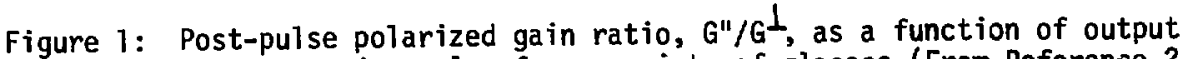
fluence of the saturating pulse for a variety of glasses (From Reference 2 with permission of the authors). 
glazs. Regardless of the scatter, an average decrease in the ratio with increasing output fluence is discernable.

The gain ratio plotted in Figure 1 is derived from what Martin and Milam call "recovered gain" values. ${ }^{4}$ Their temporally-resolved gain probe traces showed a dip below the recovered value during and imnediately after the saturating pulse for a probe with polarization parallel to that of the pulse. This signal then increased to the recovered gain value which was always less than the perpendicularly polarized probe gain. The transient was initially interpreied as being due to the details of the level population dynamics. Further analys is has shown this interpretation to be untenable. ${ }^{4}$ The source of the probe transient is still unexplained. Evidence of the transient has been observed in shots made with unpump, u aln " fier rods, implying that the phenomeron is not a result of the Nd level popuiation dynamics.

\section{B. ED-2 Parameters}

Parameters needed ;or calculating saturation in an amplifier with a given initial small-signal gain are the small-signal cross section $(\bar{\sigma})$, the level degeneracy factor $\left(1+g_{u} / g_{1}\right)$, the cross section anisotropy $(\delta)$, and the ratio of homogeneous to inhomogeneous linewidth $\left(\Delta \nu_{h} / \Delta \nu_{i h}\right)$.

It should be noted that, whenever some microscopic property of ions in a glass is described by a single number, we are dealing with some average or "effective" quantity. For example, the homogeneous linewidth of a transition of a single ion will vary from ion to ion and also from trar.sition to transition for a single ion. Our model treats all transitions as having equal homogeneous linewidths, $\Delta \nu_{h}$. The 
"effective" character of these quantities reflects the desire to keep this model, which describes the behavior of a large number of particles, as simple as possible while still able to accurately predict amplifier behavior.

The stimulated emission cross section for ind in ED-2, determined by Judd-Ofelt calculation, ${ }^{6}$ is appropriate for the small-signal cross section. This calculation, which is based on absorption and emission. . measurements, treats the medium as homogeneous and obtains a peak cross section due to the weighted contribution of all ions. The assumptions and approximations of the method are describeo by krupke. ${ }^{6}$ For ED-2, $\bar{\sigma}=2.7 \mathrm{pm}^{2}$ (Ref. 7).

\section{$1+g_{1} \underline{1 g}_{1}$}

As mentioned in Section $9-C$, the degeneracy factor may be used to account for the relaxation that occurs between the terminal laser level and the ground-state manifold as well as actual level degeneracies.

For a $\mathrm{Nd}^{3+}$ ion, free from perturbing external fields, the four states of the ${ }^{4} F_{3 / 2}$ level are energy degenerate as are the 12 states of the ${ }^{4} I_{11 / 2}$ leve1. The stimulated emission cross section between specific states of the two levels, which is a measure of the probability of emissive transition of an ion in a radiation field, equals the absorption cross section for transition between the two states. This may 
be shown by quantum mechanical calculation or by Einstein's $A$ and $B$ coefficient analysis. ${ }^{8}$ The total absorption or stimulated emission of light by transitions between all states of the levels may be characterized by a single stimulated emission cross section which is an average of the individual state-to-state cross sections and a degeneracy factor, $1+g_{u} / g_{1}=1+4 / 12=4 / 3$. The factor accounts for the fact that net absorption of light is less probable than net stimulated emission since, in absorption, a Nd ion in the lower level can go to only four upper-level states while an ion in the upper level can go to any of the 12 lower-level states. For the free ion there is no mechanism for relaxation from the ${ }^{4} I_{11 / 2}$ level to the ground level $\left({ }^{4} I_{g / 2}\right)$, except via forbidden spontaneous radiative transitions.

When a $\mathrm{Nd}^{3+}$ ion is incorporated into a low-symmetry site in a glass, its interaction with the crystal field removes all of the level degeneracy except for the Kramers' degeneracy. One may think of the upper level as made up of two distinguishable states while the lower level is made up of six. The cross sections of the individual state-to-state transitions must then be considered and again absorption and emission cross sections are equal. However, in a solid a mechanism exists for relaxation of excited states by eilission of phonons. While relaxation from the ${ }^{4} \mathrm{~F}_{3 / 2}$ level requires a multiphonon process and is relatively improbable, ${ }^{9}$ relaxation frcm the ${ }^{4} I_{11 / 2}$ level to the ${ }^{4} I_{g / 2}$ level is very probablo. Since ions that relax to tine ground state do not make absorptive transitions at the laser frequency, stimulated emission between the levels predominates over absorption 
regardless of level populations, not as a result of degeneracy, but because of lower-level population depletion. An infinitely fast relaxation time gives the same results as an infinitely degenerate lower lèvel.

The time constant for relaxation from the lower level is not precisely known but is thought to be much less than 1 ns. A value of this magnitude is substantiated by Martin and Milam's observation ${ }^{1,3}$ of identical saturation results for pulse durations of 1,10 , and 50 ns. Rudnitskij et al. ${ }^{10}$ determined an upper-bound for the time constant to be $2 \mathrm{~ns}$ by measurements of saturation of the weak ${ }^{4} I_{11 / 2} \rightarrow{ }^{4} F_{3 / 2}$ absorption associated with the small thermal population of the ${ }^{4} I_{11 / 2}$ level. Relaxation times that are short compared to the duration of the laser pulse imply a degeneracy factor of unity.

This value, combined with $\bar{\sigma}$ yield a small-signal saturation parameter, $\Gamma_{\mathrm{S}}$, of $6.89 \mathrm{~J} / \mathrm{cm}^{2}$ for ED-2.

6

An effective cross section anisotropy, defined by $\delta=1-\sigma_{s} / \sigma_{p}$; is determined from the FLN measurement of the depolarization ratio and the mieroscopic model of Section 9-8. One finds that $p=0.89 \pm 0.03$ corresponds to $\delta=0.58 \pm 0.05$ (Figure 9-2). The increased fractional error in $\delta$ results from the shailow slope of the $\rho$ vs. $\delta$ curve at this This value of $\delta$ compares favorably with those that can be inferred from polarized fivorescence measurements of transitions between the same $\mathrm{Nd}^{3+}$ leveis in the uniaxial crystal LiYF 4 . The point group symmetry 
of $\mathrm{Nd}^{3+}$ is $\mathrm{S}_{4}$ and the degeneracy of the levels is completely removed (except for the Kramers' degeneracy). In this crystalline case, all the anisotropic sites have the same orientation so that the anisotropy has a net macroscopic effect in "small-signal" experiments. Harmer, Linz, and Gabbe, ${ }^{11}$ exciting with unpolarized light, found that the spontaneous fluorescence lines of the $1.047 \mu \mathrm{m}$ and $1.053 \mu \mathrm{m}$ laser transitions are polarized, but not completely. The ratios of the peak intensities for fluorescence polarized perpendicular and parallel to the crystal's $C$ axis are $0.38(1.047 \mu \mathrm{m})$ and $9.0(1.053 \mu \mathrm{m})$. These ratios are proportional to polarized cross sections (neglecting linewidth and other effects) yielding transitions characterized by $\delta=0.62$ and $\delta=0.89$. Transitions in this crystal between the ${ }^{4} F_{3 / 2}$ and ${ }^{4} I_{g / 2}$ levels range from being completely polarized to completely unpolarized.

\section{$\Delta \nu_{h /} \Delta \nu_{i h}$}

The ratio of homogeneous to inhomogeneous linewidth is a critical parameter in the calculation and also a difficult one to assess precisely. The effect of a change in this ratio on output vs. input fluence and extraction efficiency curves may be seen in Figures 9-10 to 9-12. A room-temperature homogeneous linewidth of $25 \mathrm{~cm}^{-1}$ at mid-band $(1.064 \mu \mathrm{m})$ for ED-2 was measured by a FLN experiment, ${ }^{12}$ but we must determine a value for $\Delta \nu_{i h}$ indirectly.

A schematic representation and labelling of relevant states of a $\mathrm{Nd}^{3+}$ ion in $\mathrm{glass}$ is given in Figure $2 \mathrm{a}$. In the discussion that follows, the lowest energy state of the initial laser level of each ion, 

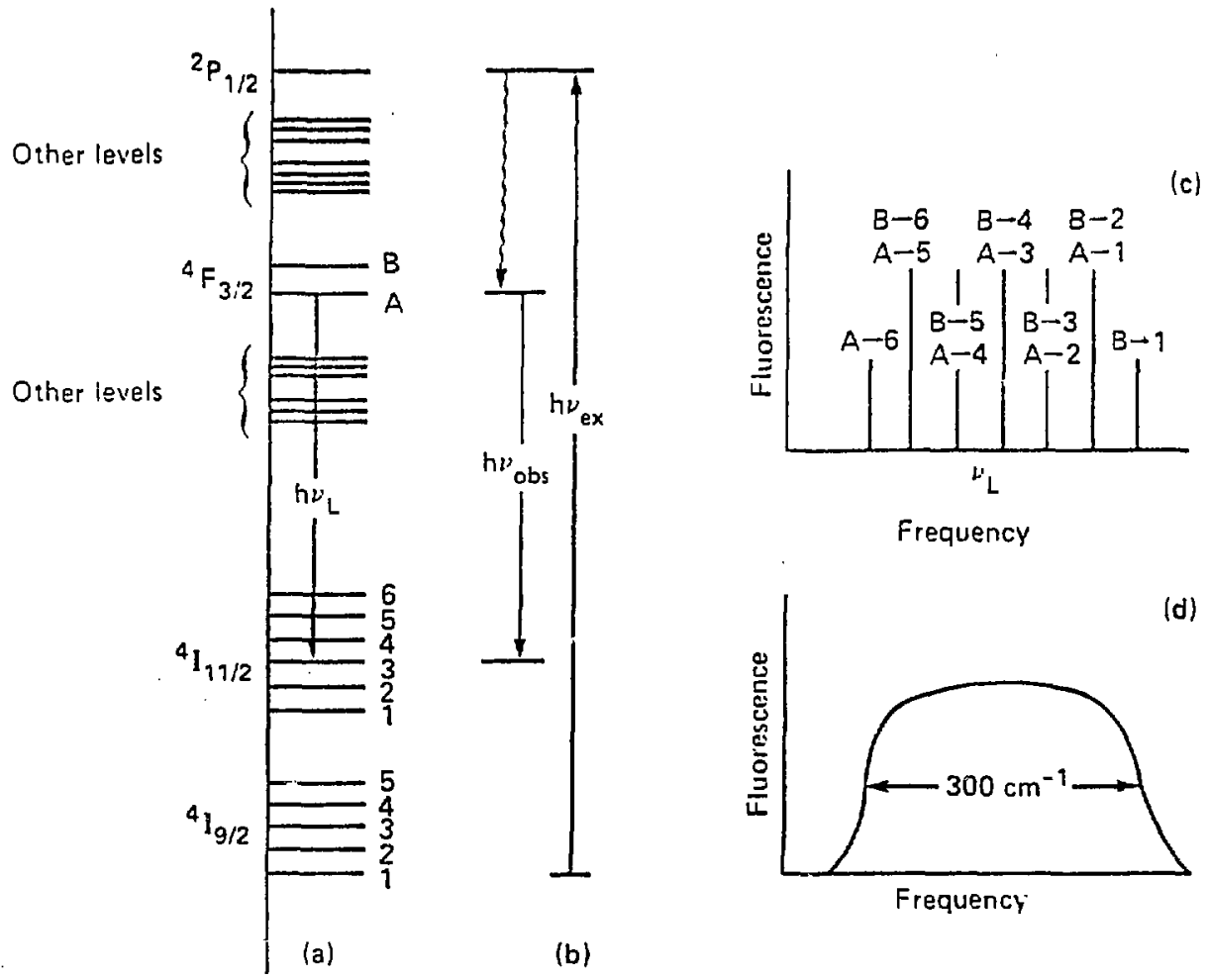

Figure 2: a) Schematic energy level diagram of $\mathrm{Nd}^{3+}$ ion in glass. b) Excitation and fluorescence scheme used by Brecher et al.12 c) Fluorescence spectrum of ${ }^{4} \mathrm{~F}_{3 / 2} \rightarrow{ }^{4} \mathrm{I}_{1 / 1 / 2}$ transitions of hypothetical $\mathrm{Nd}^{3+}$ ions. d) Spectrum of energy levels if they were inhomogeneously distributed with width on the order of the stark splitting of the "II/2 level. 
denoted by ${ }^{4} \mathrm{~F}_{3 / 2}(A)$, is at the zero of energy. Because of site-tosite variations in the crystal field, the energy of other states of a specific ion will vary about the average value depicted. This choice of zero allows the inhomogeneous linewidth of a transition involving the ${ }^{4} F_{3 / 2}(A)$ state to be associated only with the distribution in energy of the final state.

Figure $2 a$ shows that the observed width of the room-temperature broadband-excited ${ }^{4} F_{3 / 2} \rightarrow{ }^{4} I_{11 / 2}$ fluorescence line of a glass does not directly measure the inhomogeneous broadening associated with transitions between states of the two levels. The observed linewidth, $\Delta \nu_{\text {eff }}$, is due to contributions from the homogeneous linewidth and the Stark splitting of the levels, in addition to the inhomogeneous broadening resulting from a shift of an individual ion's states about average energies.

Evaluation of $\Delta \nu_{: h}$ for $\mathrm{Nd}^{3+}$ is complicated by the possibility of transitions at frequency $\nu_{L}$ between differerit states of the levels. Consider, for example, transitions orginating from the ${ }^{4} F_{3 / 2}(A)$ state and terminating on the ${ }^{4} \mathrm{I}_{11 / 2}(3)$ state. The inhomogeneous range of the ${ }^{4} I_{11 / 2}(4)$ state may allow some ions to interact via this transition. This phenomenon may be termed "transition hopping". Some ions may present comparable cross sections at $\nu_{L}$ for transitions between ${ }^{4} F_{3 / 2}(A)$ and two ${ }^{4} I_{11 / 2}$ states. Further, since transitions from both ${ }^{4} F_{3 / 2}$ states are pussible, ions in ${ }^{4} F_{3 / 2}(B)$ may make transitions at the laser frequency to other ${ }^{4} I_{11 / 2}$ states. Hence, the multi-state levels of the $\mathrm{Nd}^{3+}$ laser transition may not be precisely characterized by a single inhomogeneous distribution. 
Prefaced by these caveats, we proceed to estimate an effective inhomogeneous linewidth, $\Delta \nu_{i h}$, for ED-2. The estimate is based on the results of two experiments. The first is the liquid-helium temperature, nonresonant FLN measurements of Brecher, Riseberg, and Weber, ${ }^{13}$ in which $\mathrm{Nd}^{3+}$ ions were excited from the ${ }^{4} I_{9 / 2}(1)$ state to the singlet ${ }^{2} \mathrm{p}_{1 / 2}$ state with a tunable dye-laser (Figure $2 b$ ). Rapid nonradiative relaxation to the ${ }^{4} F_{3 / 2}$ level followed, with population of only the $A$ state being significant at $4 \mathrm{~K}$. Fluorescence to the ${ }^{4} I_{11 / 2}$ level was observed as a function of exciting laser wavelength. Structure corresponding to the $\operatorname{six}{ }^{4} F_{3 / 2}(A) \rightarrow{ }^{4} I_{11 / 2}$ transitions were observed in some glasses, although shifts in peaks due to transitions terminating on a specific ${ }^{4} I_{11 / 2}$ state could not be followed through the series of excitation wavelengths with confidence. Features were broad and poorly defined with a fluorescence bandwidth of $\simeq 300 \mathrm{~cm}^{-1}$ in $E D-2$, even though the homogeneous linewidths of the transitions are expected to be $<10 \mathrm{~cm}^{-1}$ at this temperature. Since these experiments were laser excited, one might expect six sharp spectral lines from the subset of ions selected by the laser. The broadening is a result of accidental coincidences: ions in several environments may have ${ }^{4} I_{9 / 2}(1)$ and ${ }^{2} p_{1 / 2}$ states separated by energy $h \nu_{\text {ex }}$ and ${ }^{4} F_{3 / 2}(A) \rightarrow{ }^{4} I_{11 / 2}$ energy separations that vary. This broadening may be termed "residual inhomogeneous broadening" due to imperfect site selactivity. The key resilt of the experiment is that an average Stark splitting can be discerned for the selected subset and that the total splitting is large, 
i.e. on the order of the room-temperature broadband-excited linewidth, $\Delta \nu_{\text {eff }}$. This is to be contrasted with the other possible extreme: small total stark splitting (e.g. $50 \mathrm{~cm}^{-1}$ ) combined with large inhomogeneous broadening (e.g. $250 \mathrm{~cm}^{-1}$ ) to make up the observed 307 $\mathrm{Cm}^{-1}$ effective linewidth of ED-2.

The second experiment is the $4 \mathrm{~K}$ broadband-excited fluorescence measurement of Mann and DeShazer. ${ }^{14} \mathrm{~A}$ spectrum resulting from ${ }^{4} F_{3 / 2}(A) \rightarrow{ }^{4} I_{11 / 2}$ transitions from all classes of ions in a silicate glass revealed broad features with only hints of peaks. 15 The inhomogeneous broadening is great enough to prevent resolution of transitions terminating on specific states of the ${ }^{4} I_{11 / 2}$ level, even with the overlapping transitions from the ${ }^{4} F_{3 / 2}(B)$ state eliminated by low temperature.

These two experimental results, 1) the large extent of total Stark splitting and 2) the inability to resolve transitions, combine to imply a distribution of ${ }^{4}{ }_{11 / 2}$ state energies about their average energies with widths on the order of the Stark splitting between the states.

For the purposes of our gain saturation calculations, we assume $\Delta \nu_{i h}$ is equal to the average state splitting. To obtain a value for the average splitting, imagine hypothetical $\mathrm{Nd}^{3+}$ ions in which the splittings of both the ${ }^{4} F_{3 / 2}$ and ${ }^{4} I_{11 / 2}$ levels are equal. If transition probabilities were also equal and homogeneous linewidths were small, the fluorescence spectrum from these idealized ions would be as in Figure 2c (ignoring Boltzmann populat un factors for the levels). An 
effective linewidth of $\simeq 300 \mathrm{~cm}^{-1}$ implies a Stark splitting between states of $\simeq 50 \mathrm{~cm}^{-1}$. Figure $2 d$ shows the spectrum with inhomogeneous broadening included. This rough value of $\Delta \nu_{i h}$, combined with the measured $\Delta \nu_{h}$, yields $\Delta \nu_{h} / \Delta \nu_{i h}=0.5$.

\section{ED-2 Comparison}

Normalized output vs. input fluence for a model inhomogeneous amplifier with initial small-signal gain $=6.2, \delta=0.58$, and $\Delta \nu_{h} / \Delta \nu_{i h}=0.5$ is shown in Figure 3. Included are predicted results of a homogeneous amplifier with the same gain as well as the experimental amplifier data of Martin and Milam, scaled by $\Gamma_{\mathrm{s}}=6.9 \mathrm{~J} / \mathrm{cm}^{2}$. Agreement between the experimental data and the inhomogeneous model is very good in both the small-signal and saturated gain regimes. Predictions of the homogeneous amplifier using a Frantz-Nodvik saturation parameter based on small-signal measurements overestimate the observed output by greater than $12 x$ for inputs greater than $0.5 \Gamma_{\mathrm{FN}}$.

Figure 4 shows the post-pulse gain ratio as a function of normalized fluence output for this inhomogeneous amplifier along with the experimental data,

Our model shows the same qualitative behavior as the experimental data but underestimates the magnitude of gain ratio reduction with saturation. This results from an attempt to represent sites that undoubtedly have a distribution of polarized cross section ratios by a single effective value obtained from the polarized FLN measurement. Because of accidental cöincidences of excitation levels. of ions in 


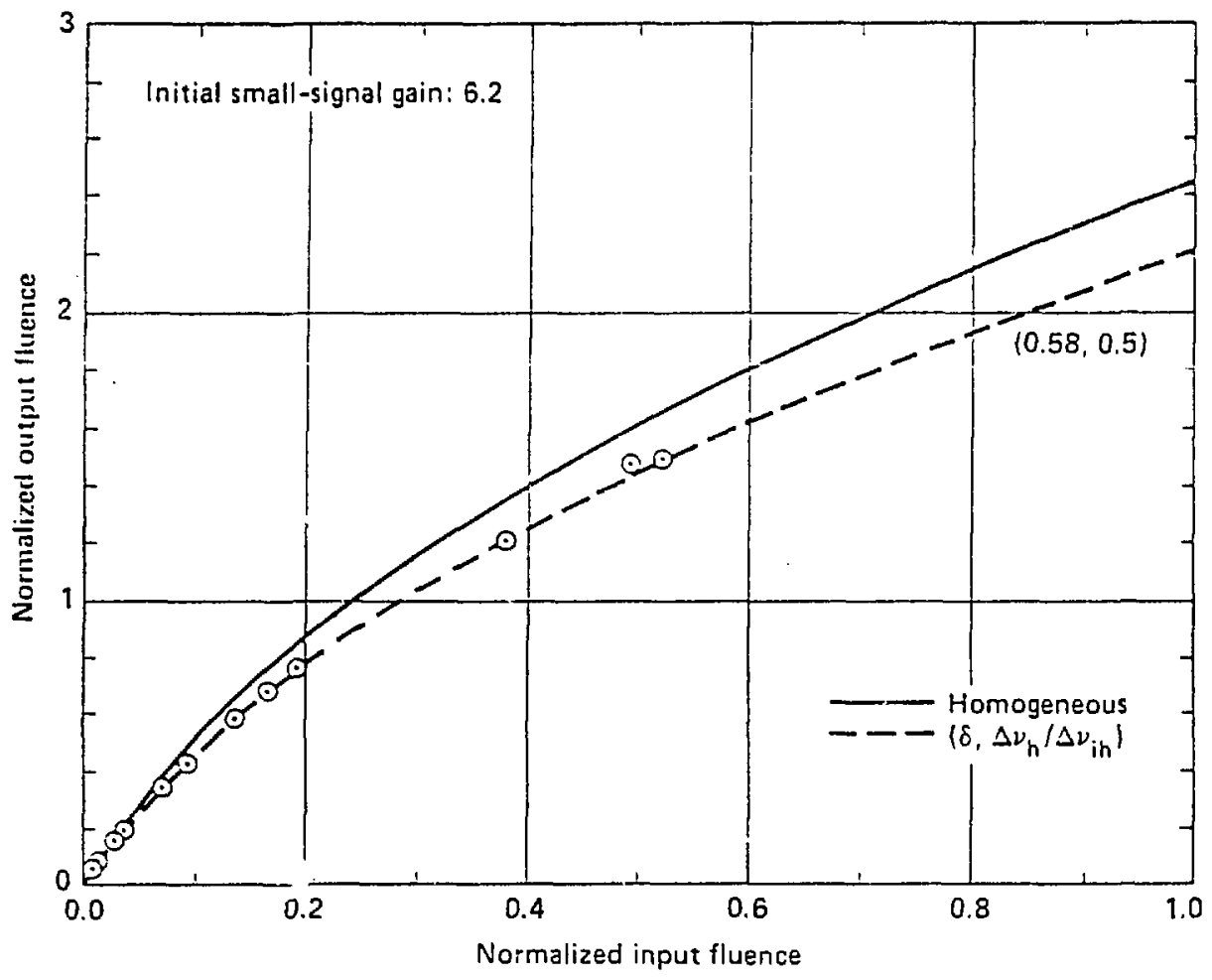

Figure 3: Normalized output fluence vs. input fluence for modei ED-2 laser glass amplifier and a homogeneous (Frantz-Nodvik) amplifier. Experimental data points (from Reference 2) are scaled by $\Gamma_{\mathrm{S}}=6.9 \mathrm{~J} / \mathrm{cm}^{2}$. 


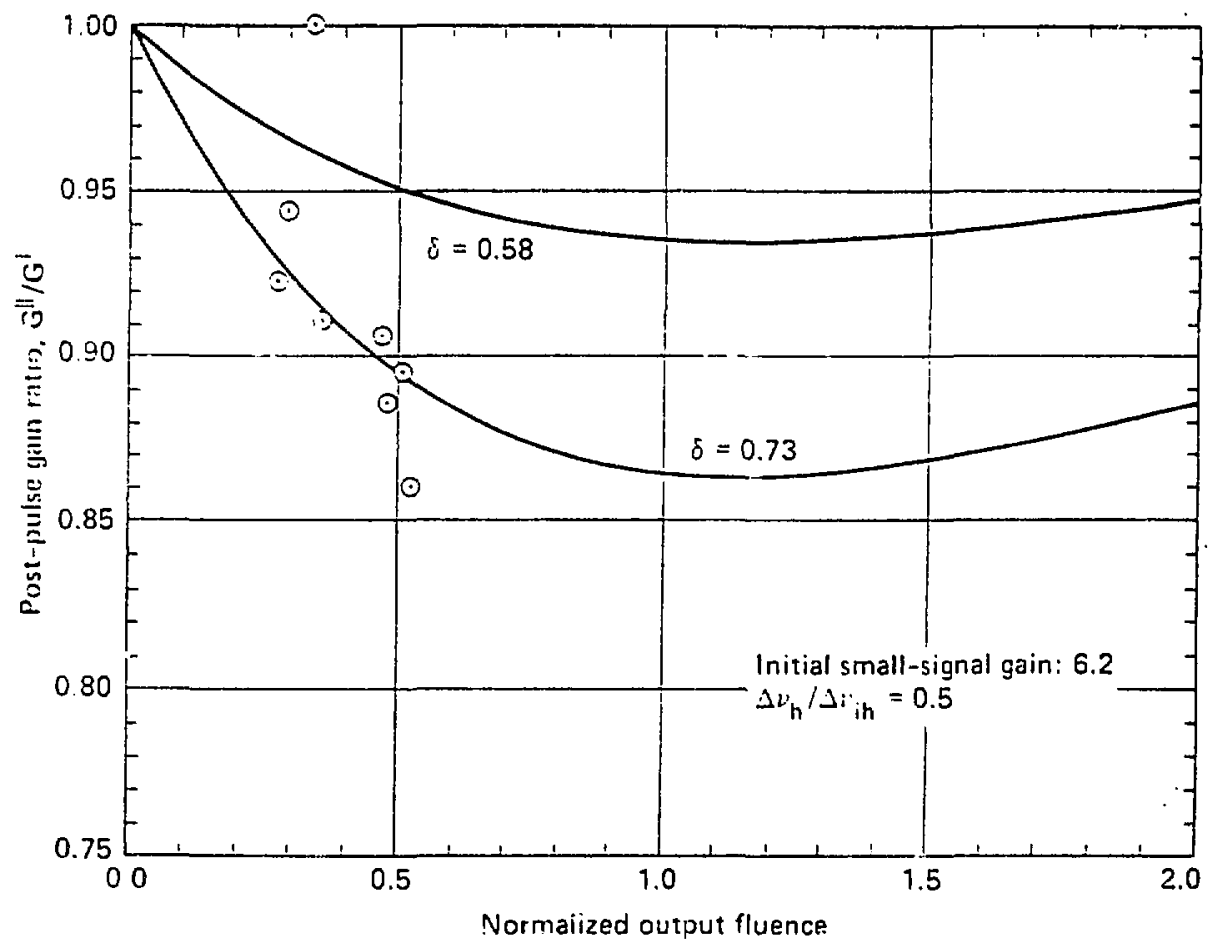

Figure 4: Post-pulse gain ratio vs. output fluence for model ED-2 glass emplifier. Data points are from Reference 2 . Also shown is behavior with "best-fit" parameters. 
physically different sites, the FLN experiment can yield oniy an effective parameter. Since there is actually a distribution of values, our model underestimates the polarization effects in an analogous way that use of an average cross section fails to account for spectral hoie burning; i.e. low $\delta$ sites burn out rapidly leaving highly anisotropic sites $(\delta \rightarrow 1)$ to be probed by the post-pulse gain measurements. The model does account ror one type of cross section inhomogeneity by allowing the cross section for microscopically identical sites to vary with the site's orientation. There are other, more subtle sources of inhomogeneity in which sites with the same orientation and spectral characteristics have different cross sections as a result of different polarized cross section ratios and line strengths.

An effective value of $\delta=0.73, \Delta \nu_{h} / \Delta \nu_{i h}=0.5$ fits the gain ratio well, but the optimum to be used to correct for the distribuition of polarized cross section ratios will vary with the degree of saturation.

The extraction efficiency of inhomogeneous and homogeneous ED-2 glass amplifiers are compared in Figure 5. For a normalized input fluence of 0.5 , only $82 \%$ of the energy extracted from a homogeneous amplifier will be sxtracted from the inhomogeneous amplifier.

\section{Q-88 Comparison}

Fluorescence line-narrowing studies of Q-88 are not as complete as those of ED-2 because of a lack of suitable excitation source with a

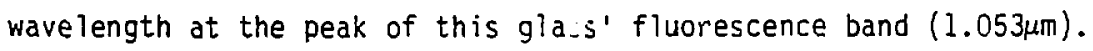
Parameters necessary for calculating the performance of a $0-88$ amplifier can be obtained by scaling ED-2 results and from other sources. 


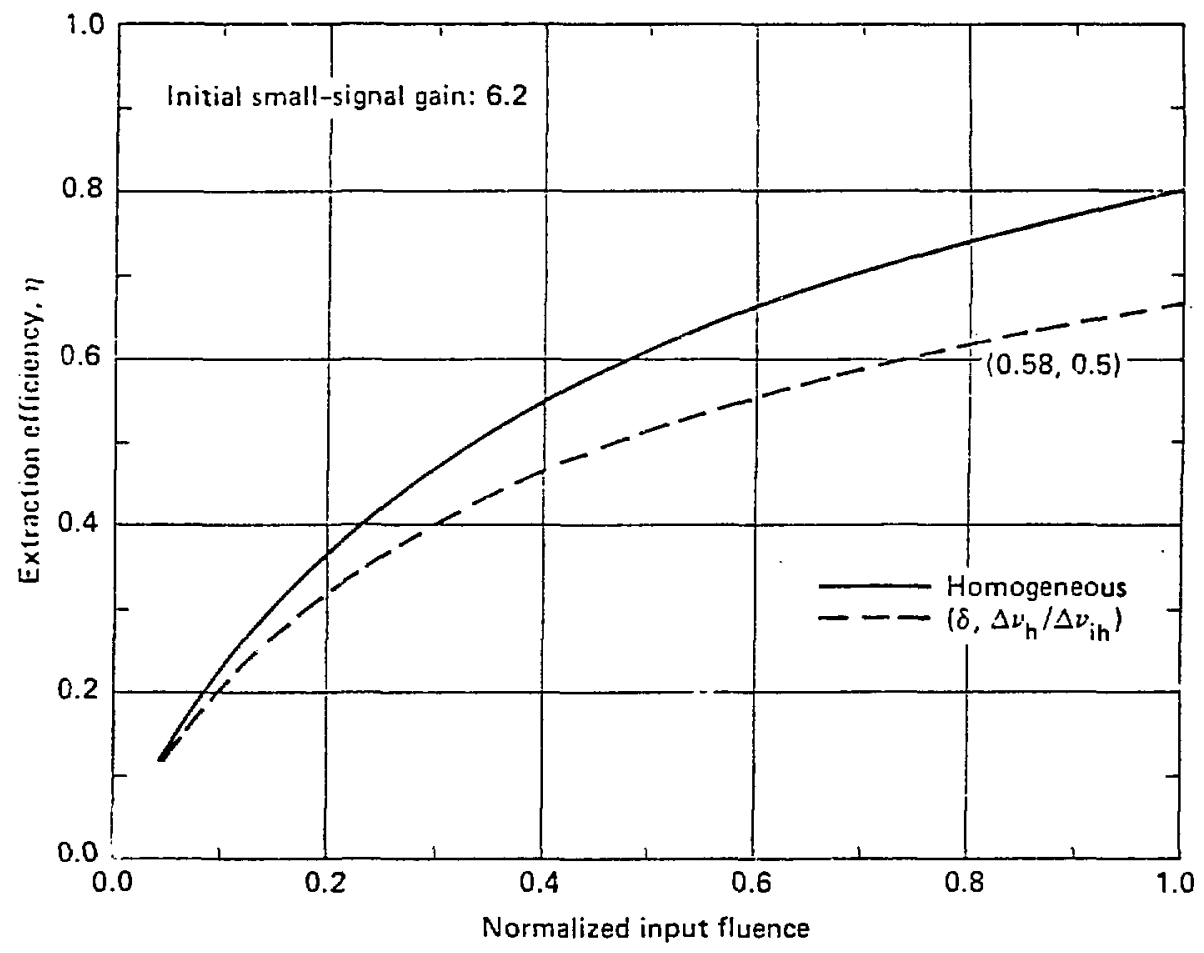

Figure 5: Extraction efficiency vs. output fluence for model ED-2 glass emplifier and homogeneous amplifier. 
The small-signal cross section of Q-88 is $4.0 \mathrm{pm}^{2}$ (Ref. 7) and, with a degeneracy factor of unity, yields a saturation parameter $\Gamma_{\mathrm{s}}=4.7 \mathrm{~J} / \mathrm{cm}^{2}$. The cross section anisotropy for this glass was not measured, therefore, in lieu of direct measurement, we take $\delta$ eqtial to that of ED-2. The observations of Lebedev and Przhevuskij $i^{16}$ of similar depolarization ratios for transitions of $\mathrm{Eu}^{3+}$ in silicate and phosphaice glasses lends weight to this assumption. A homogeneous linewidth of $\simeq 25 \mathrm{~cm}^{-1}$ for . the $1.05 \mu \mathrm{m} \mathrm{N} d^{3+}$ laser transition in phosphate $\mathrm{glass}$ may be inferred from the work of Nikitin et al. ${ }^{17}$ described in Chapter 7 , al though the glass compositions may be somewhat different.

A $\Delta \nu_{i n}$ value for $Q-88$ remains to be determined. Phosphate glass is believed to be more spectrally homogeneous than silicate glass. One qualitative indicator comes from Brecher et al., ${ }^{12}$ who find that the residual inhomogeneity of phosphate glass is smaller than silicate glass;

i.e. peaks in the nonresonant FLN spectra of phosphate glasses are narrower than in silicate glasses.

A straight-forward measurement of an irihomogeneous linewidth of a $\mathrm{Nd}^{3+}$ transition comes from the low-temperature $(4 \mathrm{~K})$ absorption measurements of Pellegrino, Yen, and Weber. ${ }^{18}$ Absorption from the ${ }^{4} I_{9 / 2}(1)$ state to ${ }^{4} F_{3 / 2}$ states yields broad but well-resolved doublets. Since the homogeneous linewidth of the ${ }^{4} I_{9 / 2}(1)$ and ${ }^{4} F_{3 / 2}(A)$ states are small, the width of the low-energy peak, labelled, $\Delta \nu_{i h}(9 / 2)$, is a direct measure of the width of the ${ }^{4} I_{g / 2}(1)$ inhomogeneous distribution. The measured silicate glasses have an inhomogeneous width $\Delta \nu_{i h}(9 / 2) \simeq 100 \mathrm{~cm}^{-1}$ while phosphate 
glasses have widths $\simeq 50 \mathrm{~cm}^{-1}$. Pellegrino et al. noted a linear correlation between the effective linewidth, $\Delta \nu_{\text {eff }}$, of room-temperature broadband excited ${ }^{4} F_{3 / 2} \rightarrow{ }^{4} I_{11 / 2}$ fluorescence and $\Delta \nu_{i h}(y / 2)$ for a wide variety of glass compositions. The linear function describing $\Delta \nu_{i h}(9 / 2)$ vs. $\Delta \nu_{\text {eff }}$ does not pass through the origin because, $\varepsilon$ en with no inhomogeneous broadening, $\Delta \nu_{\text {eff }}$ is nonzero due to Stark splitting and finite homogeneous widths (the $x$-intercept of the 1 in is at $\Delta \nu_{\text {eff }}=135 \mathrm{~cm}^{-1}$ ).

We assume that an effective value of $\Delta \nu_{i h}$ necessary for the inhomogeneous amplifier calculations scales directly with $\Delta \nu_{i h}(9 / 2)$. The linear relation between $\Delta \nu_{i h}(9 / 2)$ and $\Delta \nu_{\text {eff }}$ may be used to determine $\Delta \nu_{i h}$ from tabulated data. For $\mathrm{ED}-2, \Delta \nu_{\text {eff }}=307 \mathrm{~cm}^{-1}$, which implies $\Delta \nu_{i h}(9 / 2)=100 \mathrm{~cm}^{-1}$ from the Pellegrino et al. relationship. As we have seen, an effective inhomogeneous linewidth of $\Delta \nu_{i h}=50 \mathrm{~cm}^{-1}$ successfully predicts ED-2 amplifier data. Now, for Q-88, $\Delta \nu_{\text {eff }}=237 \mathrm{~cm}^{-1}$ implying $\Delta \nu_{i h}(9 / 2)=60 \mathrm{~cm}^{-1}$. Hence, a scaled $\Delta \nu_{i h}$ appropriate for Q-88 is $\Delta \nu_{i h}=50 \mathrm{~cm}^{-1} \times(60 / 100)=30 \mathrm{~cm}^{-1}$. This value, combined with $\Delta \nu_{h}=25 \mathrm{~cm}^{-1}$, yields $\Delta \nu_{h} / \Delta \nu_{i h}=0.83$ for Q-88.

Normalized output vs. input fluence for a model amplifier with initial small-signal gain $=7.0, \beta=0.58$, and $\Delta \nu_{h} / \Delta \nu_{i h}=0.83$ is shown in Figure 6 along with the data of Yarema and Milam scaled by. $\Gamma_{\mathrm{s}}=4.7 \mathrm{~J} / \mathrm{cm}^{2}$. Also shown are predicted results of a homogeneous amplifier. Agreement between the experimental data and the inhomogeneous model is good, especially considering $\Delta \nu_{h}$ and $\delta$ were not measured directly for this material. Figure 7 shows the post-pulse gain ratio for 


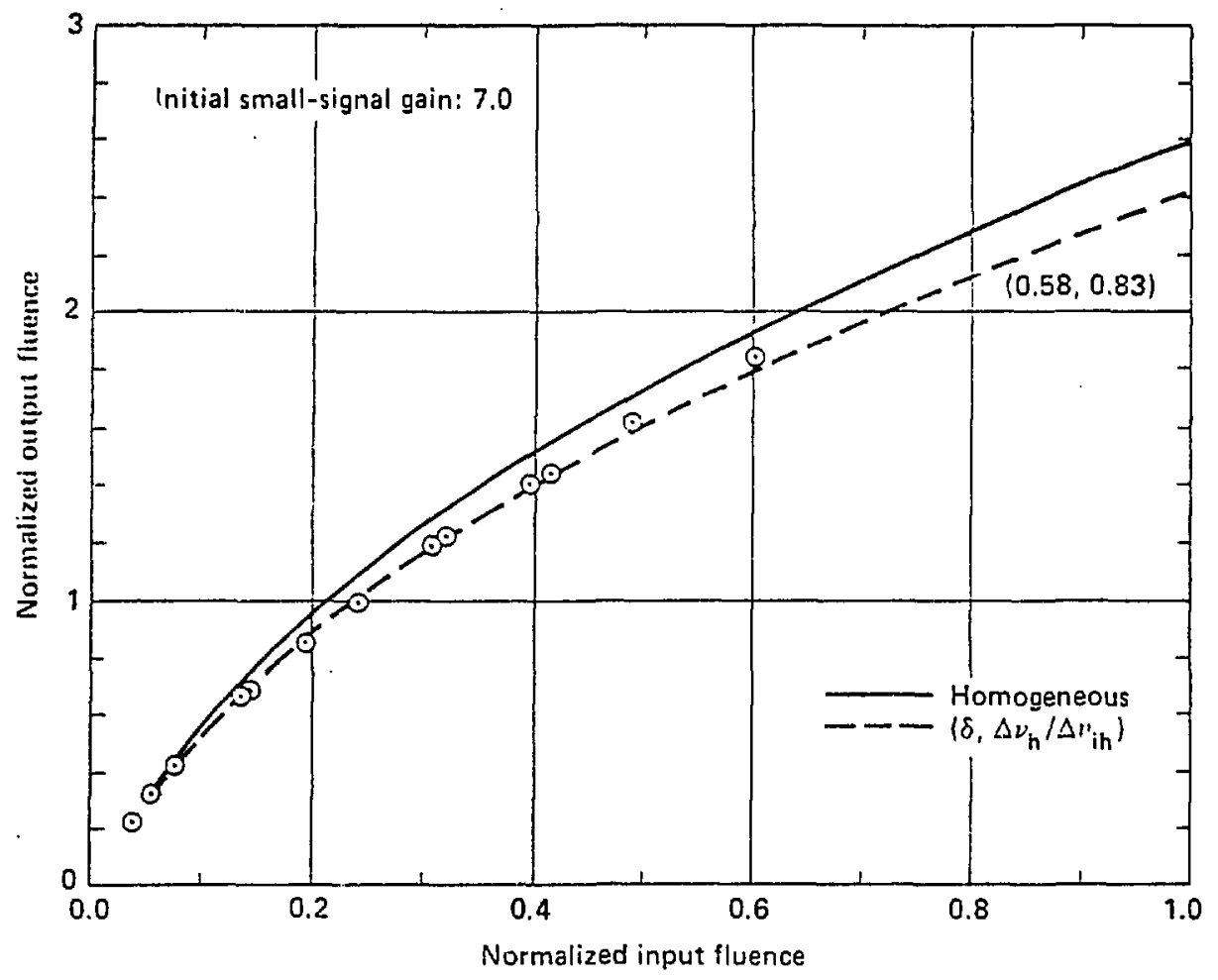

Figure 6: Normalized output fluence vs. input fluence for the model Q-88 laser glass amplifier and a homogeneous (Frantz-Nodvik) amplifier.

Experimental data points (from Reference 5) are scaled by $\Gamma_{\mathrm{s}}=4.7 \mathrm{~J} / \mathrm{cm}^{2}$. 


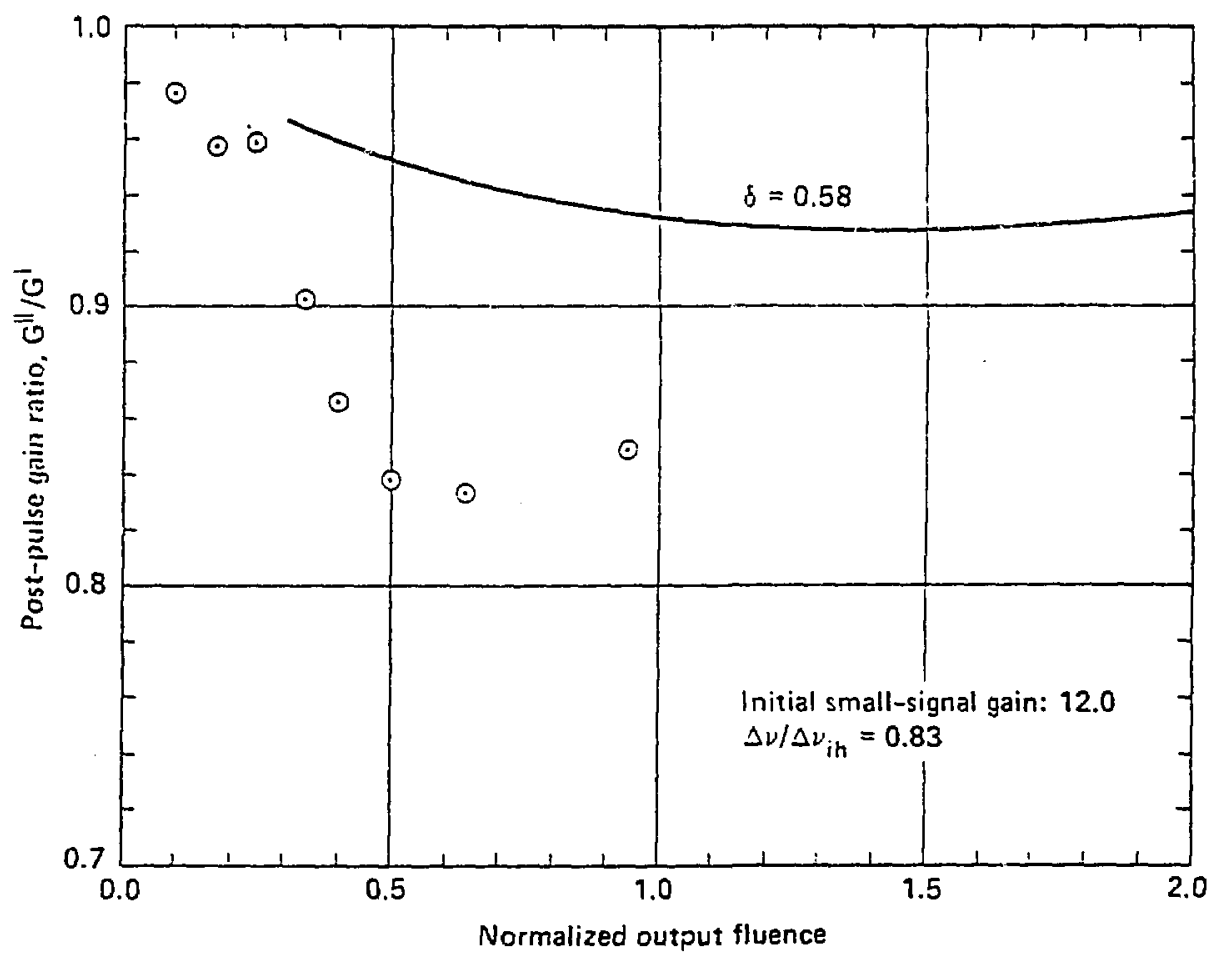

Figure 7: Post-pulse gain ratio vs. output fluence for the model Q-88 glass amplifier. Data points are from Reference 2. 
data with small-signal gain $=12.0$ which exhibits the same underestimation as in the ED-2 case. Extraction efficiency is shown in Figure 8. E. Conclusions

We have formulated a model of inhomogeneous laser media and developed codes that predict the performance of short-pulse amplifiers based on these materials. Qualitative features observed in amplifier experiments, such as a polarization dependence of the post-pulse gain, are described by the model and output vs. input fluence data may be quantitatively predicted. The model may be used to quickly evaluate the relative performance of different $\mathrm{Nd}: \mathrm{glass}$ materials with data obtained from small $\left(=1 \mathrm{~cm}^{3}\right)$ samples, obviating the need of preparing and testing actual laser amplifiers.

A major result regarding orientational inhomogeneity is that its effect on extraction efficiency is usually smali compared to the effect of spectral inhomogeneity, except in cases of extreme cross section anisotropy. This contribution to inhumogeneity is always present in glass media, however, even for laser transitions between spectrally homogeneous levels.

The model represents more than a "curve fitting" procedure in that its parameters are based on establisred physical phenomena and are related to measurable quantities that vary with glass composition. This work shows that, in order to fully evaluate a potential laser glass, one must know its homogeneous linewidth, the extent of inhomogeneous broadening, and the degree of cross section anisotropy. (In the case of long extraction pulses, one must also know the rate of cross relaxation which contributes to "hole filling"). 


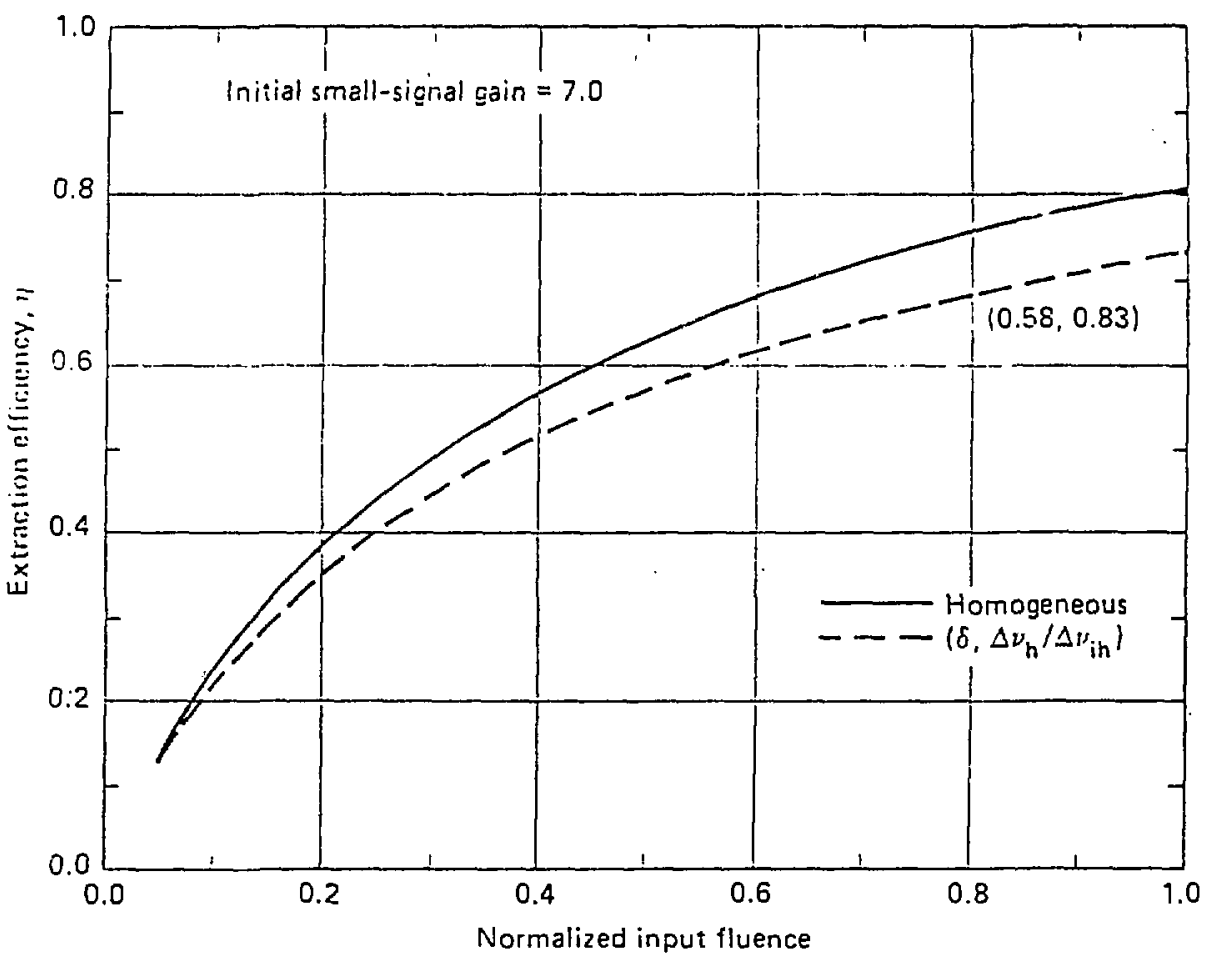

Figure 8: Extraction efficiency vs. output fluence for the model Q-88 giass anplifier and homogeneous amplifier. 
It is also evident from this work that further investigation is warranted into (1) methods of quantifying the degree of spectral inhomogeneity for multistate systems, (2) the distribution of cross section anisotropies, and (3) the measurement of FLN parameters in more and more varied glasses. 


\section{References}

1. W. E. Martin and D. Milam, "Gain saturation in Nd:doped laser materials", (to be published in IEEE J. Quant. Elect.)

2. W. E. Martin and D. Milam, LLNL UCID Report 18868 (1980).

3. W. E. Martin and D. Milam, App 1. Phys. Lett. 32, 816 (1978).

4. W. E. Martin and D. Milam, "Time-resolved gain saturation experiments in Nd:doped glasses and crystals", (in preparation).

5. S. M. Yarema and D. Milam, "Gain saturation in phosphate laser glasses", (submitted to IEEE J. Quant. Elect.)

6. W. F. Krupke, IEEE J. Quant. Elect. QE-10 450 (1974).

7. S. E. Stokowski, R. A. Saroyan, and M. J. Weber, LLNL Report M-095 (2nd revision) (1981).

8. See e.g. A. Yariv, Quantum Electronics, 2nd Ed., (John Wiley and. Sons, New York, 1975) Ch. 8.

9. C. D. Layne, W. H. Lowdermilk and M. J. Weber, IEEE J. Quant. Elect. QE-11 798 (1975).

10. Yu. P. Rudnitskii, R. V. Smirnov, and V. M. Chernyak, Sov. J. Quant. Elect. 61107 (1976).

11. M. M. Harmer, A. Linz, and D. B. Gabbe, J. Phys. Chem. Solids 301483 $(1969)$.

12. S. A. Brawer and M. J. Weber, Appl. Phys. Lett. 3531 (1979).

13. C. Brecher, L. A. Riseberg, and M. J. Weber, Phys. Rev. B 185799 (1978).

14. M. M. Mann and L. G. DeShazer, J. Appl. Phys. 412951 (1970). 
15. Mann and DeShazer attempted to fit Gaussian lines to spectra to obtain energies and widths of individual transitions. These fittings are not unique and we know that lineshapes need not be Gaussian.

15. V. P. Lebedev and A. R. Przhevuskij, Opt. Spectros. (USSR) 48513 (1980).

17. V. I. Nikitin, M. S. Soskin, and A. I. Khiznyak, Sov. J. Quantum Electron. 8788 (1978).

18. J. M. Pelligrino, W. M. Yen, and M. J. Weber, J. Appl. Phys. 516332 (1980). 


\section{Acknowledgments}

It is a pleasure to acknowledge the assistance of the individuals who contributed to this work. Foremost, I thank my supervisor, Marvin J. Weber, for sharing his enthusiasm for physics and his unequivocal expertise. His consistent support and guidance is gratefilly recognized.

Steve Brawer contributed greatly to my studies with both experimental adyice and physical insight. I thank Dave Blackburn of NBS for preparing the metaphosphate glass samples, Fred Milanovich of LLNL for the Raman spectra, and Dave Ottesen of Sandia for the IR reflection spectra. Thanks to Walter A. Hargreaves of Optovac, Inc. for the gadolinium doped fluorite samples. Jeanne Lynch and Tom Hindley provided valuable technical assistance.

Discussions with Roger Haas, Dave Milam, and especially, Travis White proved extremely usefu1. Thanks are due to Treva Janssen for her cheerful and efficient preparation of the manuscript.

Finally, I thank my wife, Janene M. Cooper, without whose support and love this project would not have been possible. This work is dedicated to the memory of Carrie Lee Cooper.

Work performed under the auspices of the U.S. Department of Energy by Lawrence Livermore National Laboratory under Contract No. W-7405-ENG-48. 
Appendix A: Kramers-Kronig Transforms

Infrared experiments are an established and valuable method for investigating the vibrational modes of solids. When the interaction between the radiation and modes is relatively strong, however, it is difficult to obtain samples thin enough for standard IR absorption experiments; information may still be extracted from reflection data. Because of the dispersion associated with reflection spectra, the quantity most indicative of the strength and frequency of IR-active vibrational modes is the imaginary part of the dielectric constant, $\epsilon_{2}$.

The theory and numerical methods necessary to obtain $\varepsilon_{2}$ (and all the optical constants) of an isotropic medium from near-normal reflectance data are presented. The keystone of the approach is the Kramers-Kronig transform which relates the imaginary part of a causal linear response function to its real part. The mathematics of the transform for a general response function is first discussed. The . electromagnetic theory required for application to reflectance data is then sketched, followed by a discussion of the specific equations used. Numerical integration of these equations, appropriate for application to vibrations in glasses, is described with emphas is on problems caused by the finite frequency domain of experimental reflectance data. A FORTRAN listing of the computer code written for our studies is included. 
A. General Response Function ${ }^{1,2}$

A linear response function loften called a generalized susceptibility) may be used to describe the macroscopic response of a system to some perturbing field; e.g. electromagnetic, electron and neutron probes. The response function, $G$, is defined by the integral equation

$$
X(r, t)=\int_{-\infty}^{\infty} d \bar{r}^{\prime} \int_{-\infty}^{\infty} d t^{\prime} G\left(\bar{r}, \bar{r}^{\prime}, t^{\prime}, t^{\prime}\right) F\left(\bar{r}^{\prime}, t^{\prime}\right),
$$

where $X$ is the response of the system and $F$ is a generalized "force" function that describes the probe field. All functions in Eq. (1) are real. The response at $\bar{r}$ and at time $t$ can be caused by a force applied. at other locations and times.

Much more may be known about the form of $G$ if some of the usual assumptions and approximations are made explicit. If the spatial variation of the probe field is small in the microscopic scale of the system, the iocat approximation may be adopted in which the macroscopic response at $\bar{r}$ is assumed due only to the force at $\bar{r}$. In this case, appropriate for optical and infrared radiation, dependence of $G$ on position may be dropped. The assumption that there is no absolute origin for time requires that $G$ depend on $t$ and $t$ ' in the functional combination $t$ - $t^{\prime}$. Equation (1) may then be written as

$$
X(t)=\int_{-\infty}^{\infty} d t^{\prime} G\left(t-t^{\prime}\right) F\left(t^{\prime}\right)
$$


Also, if we require the system to be causal (i.e. no response may occur before application of a force), then

$$
G\left(t-t^{\prime}\right)=0, \quad t<t^{\prime},
$$

To see this, consider a $\delta$-function force applied at time $t_{0}$, which yields

$$
X(t)=G\left(t-t_{0}\right)
$$

The usual response function of susceptibility discussed in optical spectroscopy is not the function $G(t)$ but is, instead, its Fourier transform, $g(\omega)$, defined by

$$
g(\omega)=\int_{-\infty}^{\infty} G\left(t-t^{\prime}\right) e^{i \omega\left(t-t^{\prime}\right)} d t .
$$

Fourier transformation of Eq. (2) deconvolves this integral equation into a multiplicative relation between $x(\omega)$ and $f(\omega)$, the Fourier transforms of $X$ and $F$, with $g(\omega)$ a proportionality constant. This form of the response function is appropriate for most experiments in which probes are quas.imonochromatic. Note that $g$ will now be complex (i.e. $g=$ $\left.g_{1}+i g_{2}\right)$ to allow for phase differences between the sinusoidal response and force functions.

In order to arrive at the kramers-Kronig transform, the domain of the complex response function is extended to the entire complex frequency 
plane. We make two assumptions (to be justified later): i) $g$ is analytic in the upper half of the complex $\omega-p l$ ane and, ii) $|g| \rightarrow 0$ when $|\omega| \rightarrow \infty$ in this portion of the plane. Then Cauchy's integral theorem,

$$
g(\omega)=\frac{1}{2 \pi i} \oint \frac{g\left(\omega^{\prime}\right) d \omega^{\prime}}{\omega^{\prime}-\omega}
$$

may be written in modified form:

$$
g(\omega)=\frac{1}{\pi^{i}} P\left[\int_{-\infty}^{\infty} \frac{g\left(\omega^{\prime}\right) d \omega^{\prime}}{\omega^{\prime}-\omega}\right],
$$

where $P$ denotes the principle part of the integral along the real axis. Assumption $i i)$ has allowed us to neglect the contribution to the integral from the hemispherical portion of the usual closed contour in the upper half plane. ${ }^{2}$ Equation (7) may be split into real and imaginary parts providing the connection between real and imaginary parts of $g$ :

$$
\begin{gathered}
g_{1}(\omega)=\frac{1}{\pi} p\left[\int_{-\infty}^{\infty} \frac{g_{2}\left(\omega^{\prime}\right) d \omega^{\prime}}{\omega^{\prime}-\omega}\right] \\
g_{2}(\omega)=-\frac{1}{\pi} p\left[\int_{-\infty}^{\infty} \frac{g_{1}\left(\omega^{\prime}\right) d \omega^{\prime}}{\omega^{1}-\omega}\right]
\end{gathered}
$$

Real and imaginary parts of $g$ are not independent; knowledge of one part of a complex response function over the entire real frequency axis permits calculation of the other part. 
Returning to our assumptions, we first ask why $g$ should be analytic in the upper half plane? Causality, with a bit of physical reasoning, guarantees it. To see this, let $\omega=\omega_{1}+i \omega_{2}$, write out Eq. (5) explicitly, and consider the terms that make up the integral:

$$
g(\omega)=\int_{-\infty}^{\infty} d t e^{i \omega_{1}\left(t-t^{\prime}\right)} e^{-\omega_{2}\left(t-t^{\prime}\right)} G\left(t-t^{\prime}\right) .
$$

The first exponential is bounded everywhere with no singularities. If we restrict ourselves to the upper half of the $\omega$-plane, the second exponential is bounded for $t>t^{\prime}$. When $t<t^{\prime}$ this term diverges; however, $G\left(t-t^{\prime}\right)=0$ for $t<t^{\prime}$ as required by causality. If we make the physical assumption that we cannot create an infinite response, we see that the integral for $g$ converges. An additional physical assumption that the response to a delta function force dies out in time makes the integral uniformly convergent by the Dirichlet-Hardy test. ${ }^{3}$ Uniform convergence allows differentjation under the integral sign and a confirmation of analyticity by the Cauchy-Riemann relations. ${ }^{4}$ The poles in the response function of a "lossy" system occur in the lower half plane. ${ }^{2}$

The validity of the second assumption must be confirmed for each specific response function and, in the case of the reflectivity response function we will consider, this assumption has been the subject of a good deal of investigation. 5,6 


\section{B. Electromagnet ic Preliminaries}

Consider an electromagnetic plane wave impinging normally from vacuum onto a semi-infinite medium whose dielectric properties are described by a complex index of refraction, $\hat{n}(\omega)=n+i k$. Maxwell's equations require that certain boundary conditions be met by the complex amplitudes that describe the electric field, E, and magnetic fieid, H. Specifically,

$$
\begin{aligned}
& E_{i}+E_{r}=E_{t}, \\
& H_{i}-H_{r}=H_{t},
\end{aligned}
$$

where the subscripts $i, r$, and $t$ represent the incident, reflected, and transmitted components of the total field. In a vacuum (in Gaussian units) $E=H$ while in the medium (assumed to be nonmagnetic so that $\mu=1$ ) $E=n H$. Substituting this result into Eq. (10) and solving for the ratio of $E_{r} / E_{i}$, we obtain

$$
r \equiv \frac{E_{r}}{E_{i}}=\frac{1-\hat{n}}{1+\hat{n}} .
$$

The reflectivity, $r$, (sometimes called the "field reflectance") is complex and a function of frequency.

A near-normal reflectance experime, ?asures the reflectance (sometimes called the power reflectance), $R$, which is related to $r$ by the relation $R=r^{*}$. The real part of $r$ is simply related to $R$. Define the reflectivity amplitude, $\rho$, and the field phase shift, $\theta$, by 


$$
r=\rho e^{i \theta} .
$$

We can see that the square of the reflectivity amplitude equals the measurable $R$.

Now take the logarithm of Eq. (12):

$$
\begin{aligned}
\ln r & =\ln \rho+i \theta \\
& =\ln \sqrt{R}+i \theta
\end{aligned}
$$

If In $r$ qualifies as a response function to which a Kramers-Kronig transform may be applied, then by knowing the experimentally accessible $R$ spectrum we can obtain $\theta$ and, hence, $r(\omega)$. Inverting Eq. (11) gives the complex index of refraction from which all optical constants may be obtained. For example, the imaginary part of the dielectric constant,

$$
\begin{aligned}
\epsilon(\omega) & =\epsilon_{1}+i \epsilon_{2}, \text { is given by } \\
\epsilon_{2} & =2 n k .
\end{aligned}
$$

The spectrum of $\epsilon_{2}$ is a physica?ly important quantity since power absorption per unit volume is given by

$$
\begin{aligned}
\frac{d E}{d t} & =\operatorname{Re}\left(\bar{E} \cdot \frac{\partial \bar{D}}{\partial t}\right) \\
& =\omega \epsilon_{2}|E|^{2}
\end{aligned}
$$

for monochromatic fields. 


\section{Reflectance Transform}

Unfortunately, in $r$ does not automatically qualify for kramers-Kronig transformation because it does not meet the second condition of Section A; i.e. as $|\omega|-\infty$, we expect $r \rightarrow 0$ which implies that $|\ln r|-\infty$. Additional investigation 5,6 does justify application of the transform to this function, however. Thus, Eq. (8) may be written as

$$
\theta(\omega)=-\frac{1}{\pi} P\left[\int_{-\infty}^{\infty} \frac{\frac{1}{2} \ln R\left(\omega^{\prime}\right) d \omega^{\prime}}{\omega^{\prime}-\omega}\right]
$$

We know that in $R$ must be symmetric about the origin since the inverse Fourier transform of the function $g$ must be real. This fact allows expression of the integral of Eq. (16) over only positive frequencies. Also, note that the integrand of $E q .(16)$ with $R(\omega)$ substituted for $R\left(\omega^{2}\right)$ is analytic at $\omega-\omega^{\prime}$, as can be seen by an application of l' Hospital's rule, and that Cauchy's theorem requires that the integral equal zero. Thus, we can subtract in $R(\omega)$ from the numerator without changing the value of the phase shift. Equation (16) may now be written in a form amenable to numerical integration:

$$
\theta(\omega)=\frac{\omega}{\pi} \int_{0}^{\infty} \frac{\left(\ln R\left(\omega^{\prime}\right)-\ln R(\omega)\right)}{\omega^{2}-\omega^{12}} d \omega
$$

Inclusion of the $R(\omega)$ term eliminates any question of scaling of reflectance since the difference of the logarithms of two numbers is equal to the logarithm of their quotient. Note that Eq. (17) is dimensionless so any convenient frequency unit may be used. 


\section{Numerical Integration}

An inspection of Eq.(17) for the field phase shifi at a given frequency reveals that a knowledge of reflectance at all frequencies is required. Since this is experimentally impossible, values for the unmeasured portion of the spectrum must be extrapolated. Fortunately, for optical constants attributable to the motion of atoms in a wide-band-gap solid and a reasonable range of infrared reflectance data, this is not difficult. Values of the static or DC dielectric constant for many materials are published ${ }^{7}$ so that reflectance at $\boldsymbol{\omega}=0$ may be computed using a formula appropriate for lossless media,

$$
R=\left[\frac{\sqrt{\epsilon}-1}{\sqrt{\epsilon}+1}\right]^{2}
$$

Various paths for joining this value with the first optically measured value may be chosen. Perhaps the best method for determining the lowest frequency value of $\theta$ to trust is to use many paths and determine that frequency for which change in $\theta$ with path is sma11. For example, in our experiments, the lowest measured reflection data point was $170 \mathrm{~cm}^{-1}$; no significant change in the optical constants occurred above $250 \mathrm{~cm}^{-1}$ when widely varying reflectances were substituted for the unmeasured low-frequer.sy region.

Values for reflectance in the unmeasured optical region may be calculated if the refractive index of the material is known. For frequencies below the intrinsic electronic transition frequency, $R$ is fairly constant. In fact, in our experiments where the highest measured 
frequency was $4000 \mathrm{~cm}^{-1}$, we obtained good results by assuming constant reflection at the $4000 \mathrm{~cm}^{-1}$ value for all higher frequencies. Other physically reasonable high-frequency boundary conditions had little effect on optical constants below $2000 \mathrm{~cm}^{-1}$. There is an increase in reflectance at $\simeq 3600 \mathrm{~cm}^{-1}$ in our spectra attributable to water in the glass. Reflectance will decrease to a value commensurate with the optical index of refraction by $\simeq 4500 \mathrm{~cm}^{-1}$. Choosing the locally increased value at $4000 \mathrm{~cm}^{-1}$ to extend to infinite frequency can make a slight difference in the value of $\theta$ below $2000 \mathrm{~cm}^{-1}$. For example, we found that $\epsilon_{2}$ (an intrinsically positive quantity) was calculated to be slightly negative in some portions of its spectrum. Choosing a ?ower value of reflectance at $4000 \mathrm{~cm}^{-1}$ eliminates this problem. The large value of reflectance that accompanies electronic transitions in the vacuum UV region of the spectrum gives little contribution to the phase shift integral at low-frequencies because of the denominator in Eq.(17).

A Kramers-Kronig transform routine, called KRAM, was written to obtain the optical constants of metaphosphate glass from near-normal reflectance spectra. Reflectance data for the frequency range $0-4000$ $\mathrm{cm}^{-1}$ were entered into an array (after digitization of the original data traces) consisting of 2000 equally spaced data points.

Trapezoidal rule integration ${ }^{8}$ was used except for the increment in which $\omega^{\prime}=\omega$. For this interval, 1 'Hospital's rule was used to evaluate the integrand and the contribution to the integral approximated by a two-unit wide rectangle. This rule requires knowledge of the derivative of reflectance at $\omega$ and this was obtained using a second order backwards 
difference representation. ${ }^{9}$ The contribution to the phase shift at for constant reflectance from $\omega_{m} \rightarrow \infty$, where $\omega_{m}$ is the maximum measured frequency, can be determined analyticaliy and is

$$
\Delta \theta(\omega)=\frac{1}{2 \pi} \ln \left[\frac{R(\omega)}{R\left(\omega_{m}\right)}\right] \ln \left[\frac{\omega_{m}+\omega}{\omega_{m}-\omega}\right] .
$$

This expression was evaluated and added to the integral for the $0-4000$ $\mathrm{cm}^{-1}$ interval.

The program was checked by creating "synthetic" reflectance data. Using the classical Lorentz oscillator mode ${ }^{10}$ with four oscillators of different frequencies and strengths, a complex dielectric constant was easily constructed. Reflectance of this model system was calculated from this $\epsilon$ using the usual equations. This reflectance was then inputted to KRAM which calculated $\epsilon$. There was no discernable difference between the dielectric constant computed by KRAM and the original synthetically produced $\epsilon$. 
E. Program, Variables, and Routines

YPLOT (2001)

$Y(2000)$

THETA(2000)

$x(4)$

CMPLXR

D

CMPLXN

DICNST

NTRANS

IOFILE

ALPHA

REFATK

REFKMI
Stores data for graphics routine.

Stores inputted reflectance, reflectivity during the transformation, and optical constants evaluated after transformation.

Stores caiculated phase shift.

Array necessary to define $X$-axis for graphics routine.

Stores reflectivity at a given frequency for calculation of optical constants.

Stores THETA when needed in complex form.

Stores complex index of refraction calculated from CMPLXR.

Stores complex dielectric constant calculated from CMPLXN.

Stores maximuin number of data points for which transform integral will be performed. If maximum reflectance data is $4000 \mathrm{~cm}^{-1}$ (point 2000), one should only trust the transform to $\simeq 2000 \mathrm{~cm}^{-1}$ (point 1000); there is no need to transform past point 1000 , so let NTRANS $=1000$. The transform is performed rrom point 10 to NTRANS with phase shift of points 110 made equal to that of 10 ; this eliminates some numerical problems.

User written subroutine to facilitate file input and output.

Exponential decay constant when reflectance past $\omega_{m}$ is assumed to be of form $R\left(\omega_{m}\right) \exp (-\alpha)$. ALPHA $=0$ implies constant reflectance to infinite frequency.

Stores reflectance at point $K\left(\omega^{\prime}=\omega\right)$ necessary for $1^{\prime}$ Hospital's rule.

Stores reflectance at point $K-1$ necessary for derivative evaluation. 
$-210-$

WKSQMI

WKSQP I

$S$

THHIGH
Stores frequency squared of $\mathrm{K}-1$.

Stores frequency squared of $k+1$.

Stores summation that approximates phase shift integral.

Stores value of phase shift attributable to unmeasured high-frequency portion of reflectance. 


\section{References}

1. F. 0. Wooten, Optical Properties of Solids, (Azademic Press, New York, 1972) Ch. 6.

2. M. Cordona, in Optical Properties of Solids, S. Nudelman and S. S. Mitra, Eds. (Plenum Press, New York, 1969) p. 137-51.

3. H. Jeffreys and B. Jeffreys, Methods of Mathematical Physics, 3rd Ed.; (Cambridge University Press, 1978) p. 46.

4. Ibid., p. 333.

5. Ref. 1, Appendix $G$

6. G. Andermann, Ph.D. Dissertation, University of Southern California, 1965, (University Microfilms, Inc.).

7. e.g. Handbook of Chemistry and Physics, 56th Ed., (CRC Press, Cleveland, 1975) E58-60.

8. R. W. Hornbeck, Numerical Methods, (Quantum Publishers, New York, 1975) p. 144.

9. Ibid., p. 20.

10. Ref. 1, p. 42. 


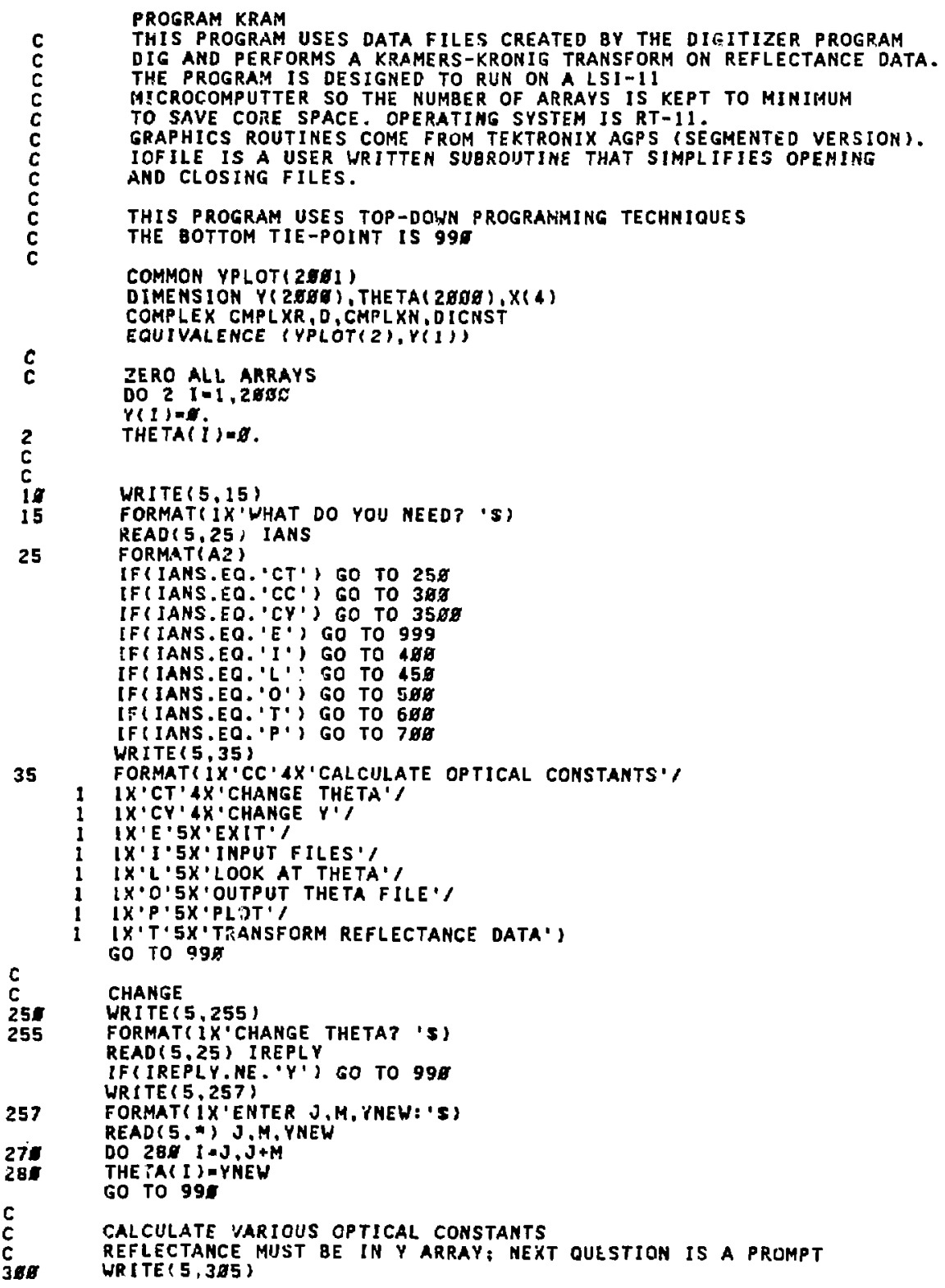




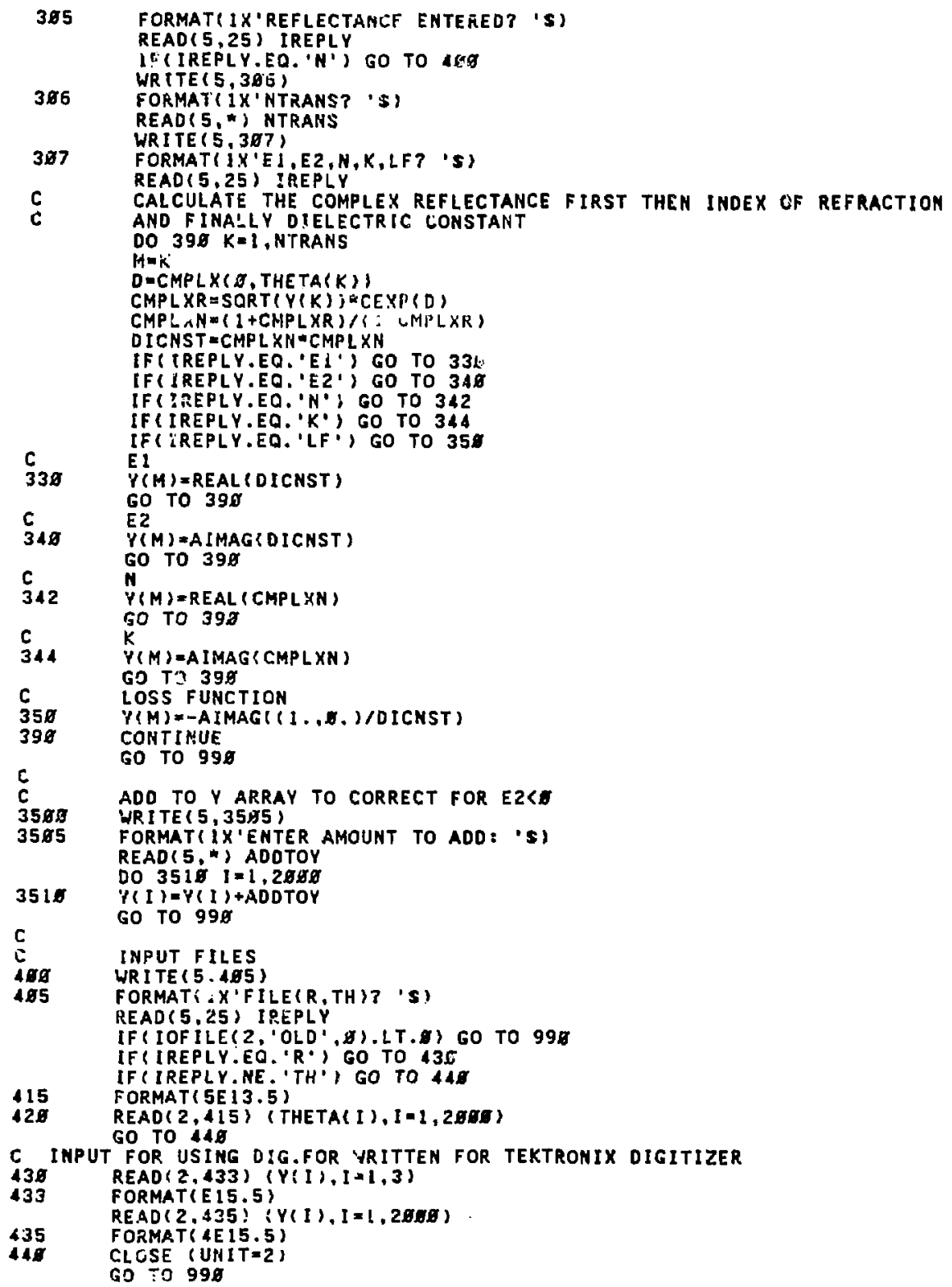




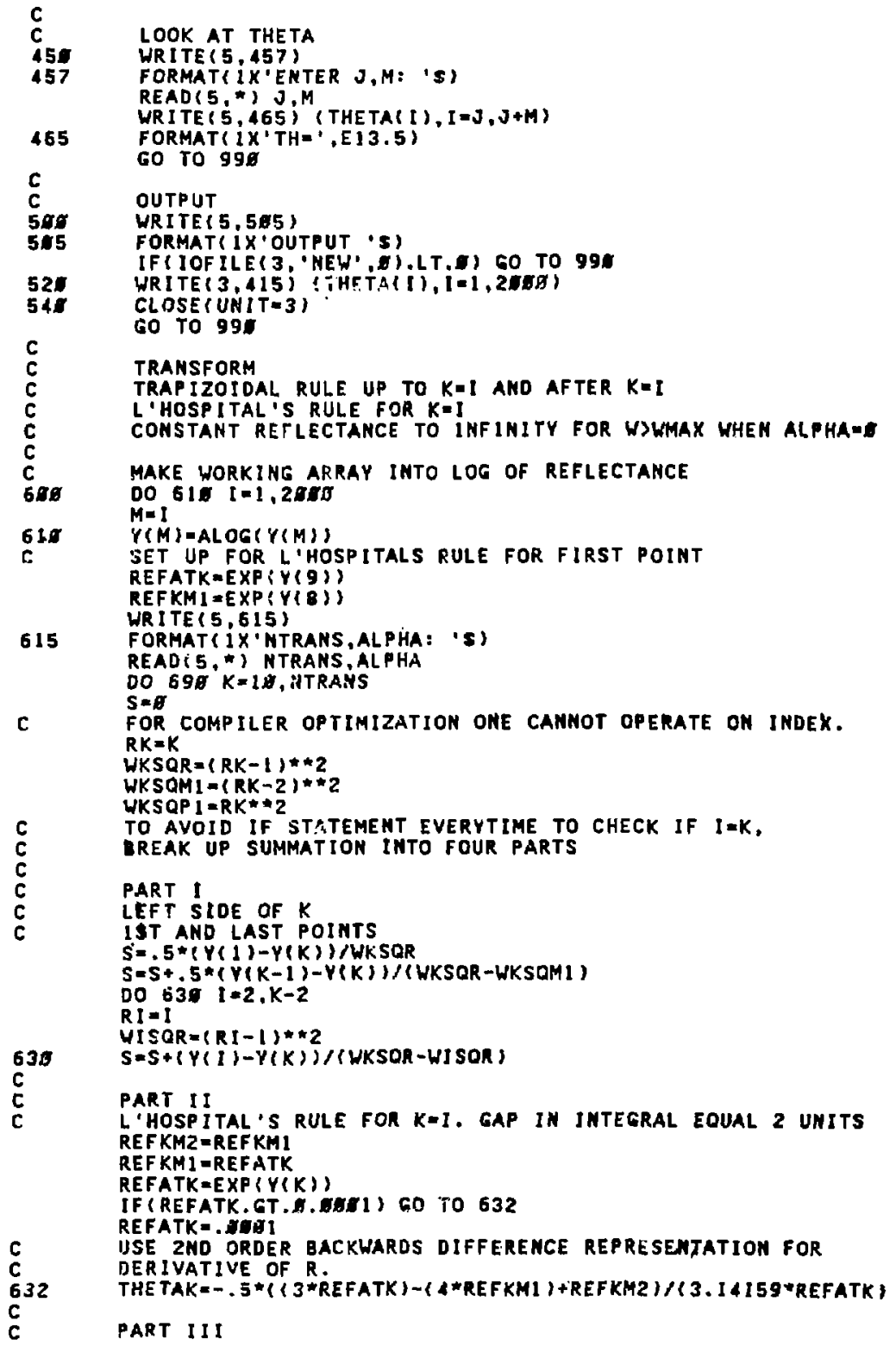




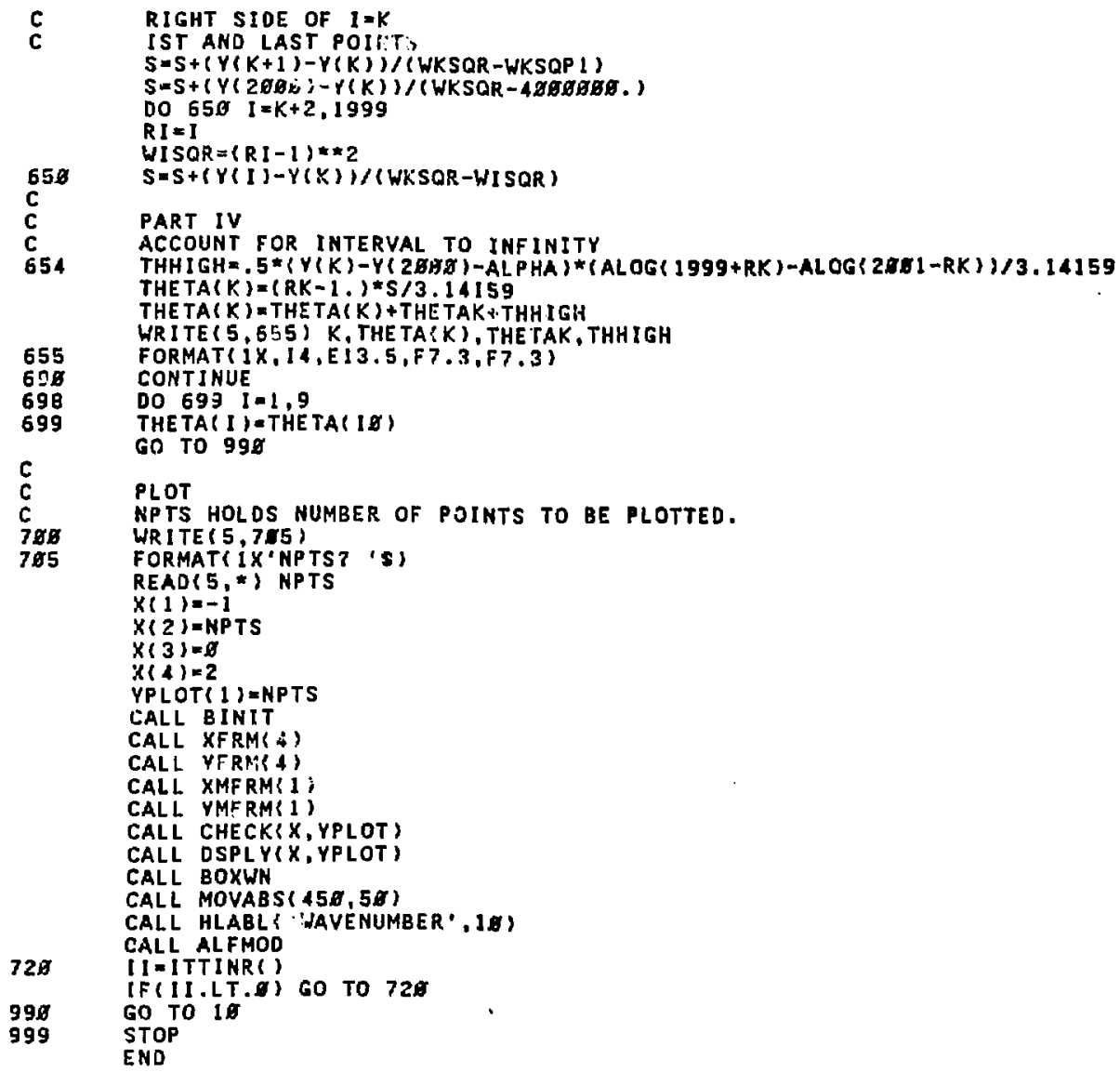




\section{Appendix B: Amplifier Calculation Computer Codes}

Programs for amplifier calculations of Chapter 9 in media with the orientational inhomogeneity (ORHOL), spectral inhomogeneity (SPHOL), and the combined inhomogeneity (HOL) follow: 


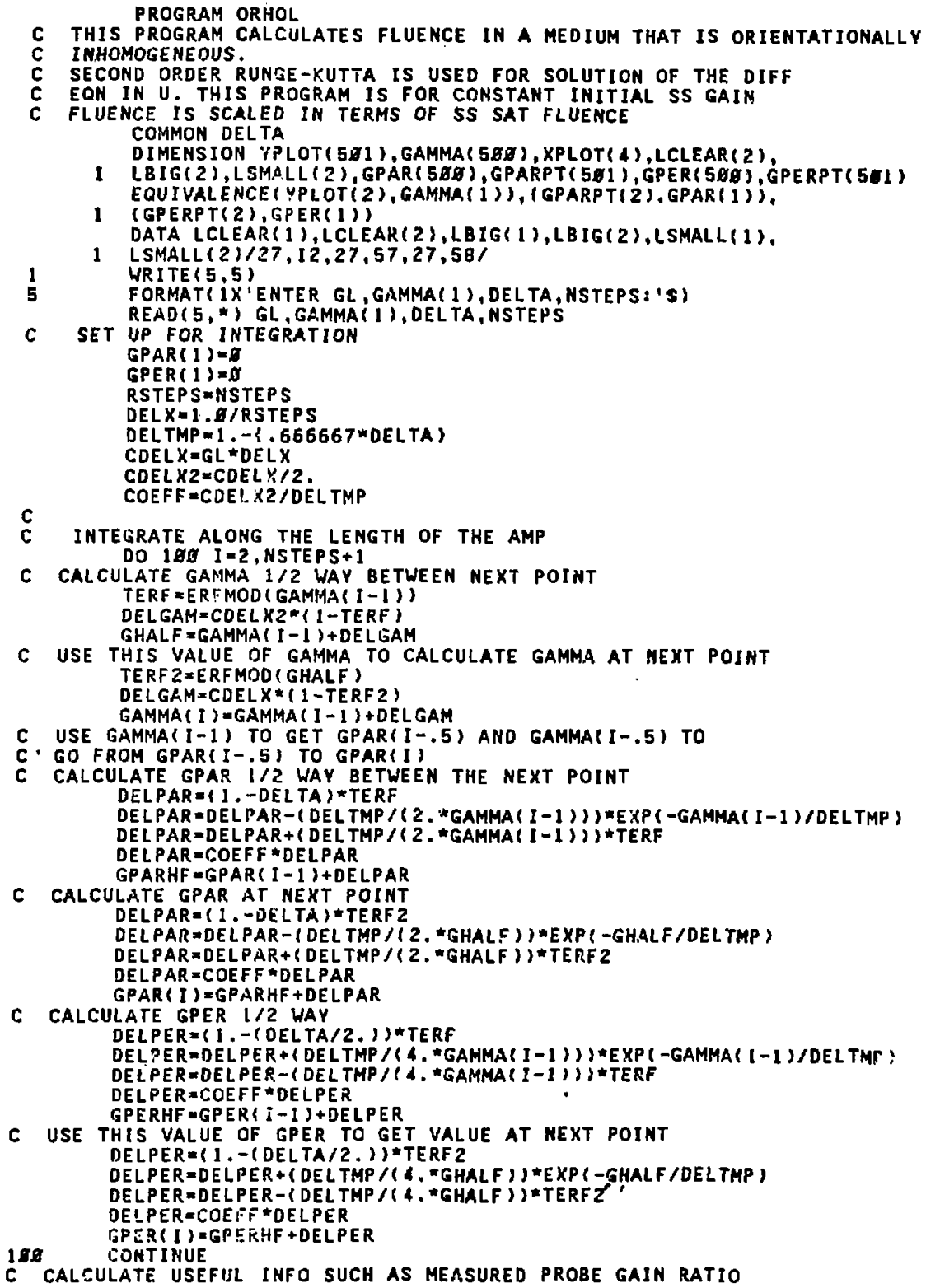


GRATIO=EXP(GPAR(NSTEPS+1) )/EXP(GPER(NSTEPS+1)

C CALCULATE RESULTS FOR FRANTZ NODVICK SYSTEM AND COMPARE FNOUT $=A L O G\{(\operatorname{EXP}(G L) *(\operatorname{EXP}($ GAMMA: 1$))-1))+1)$ FNGAIN =F NOUT-GAMMA 1 )

F MRE L $=($ GAMMA ( NSTEPS + 1 )-GAMMA( 1) )/FMGAIN

C PLOT RESULTS CALL TOUTST( 2, LCLEAR )

WRI TE (5, 115 ) GL, GAMMA ( 1$)$, GRATIO, GPER( NSTEPS+1), NSTEPS, DELTA,

1 GAMNIA (NSTEPS + 1), FNREL, GPAR ( HSTEPS +1)

115 FORMATIIX'ORIENTATIONAL HOLE-BURHING: SCALED WITH SMALL

1 SIGNAL SAT FLUENCE'/

1 IX'GAIN"LENGTH=',F5,2,5X, 'INITIAL FLUENCE='F8.4,

$15 X$, 'PPGN RATIO=',F6.3,5X,' 'LN(GPER)=',FB.4,5X, 'NSTEPS=', I4.

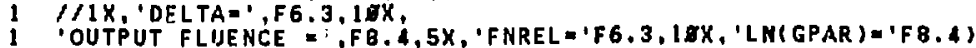
YPLOT ( 1 ) a NSTEPS +1

GPERP T (1) 1 YPLOT $(1)$

GPARPT(1)=YPLOT(1)

XPLOT( 1$)=-1$

XPLOT $(2)=Y P L O T(1)$

XPLOT $(3)=\sigma$.

XPLOT ( 4$)=D E L X$

CALL BIHIT

CALL YZERO\{D

CALL CHECK\{XPLOT, YPLOT

CALL DSPLY XXPLOT,YPI.OT

CALL TOUTST(2,LBIG)

CALL MOVABS $(40,60)$

CALL HLABL\{'LENGTH', 6 )

CALL MOVABS( 5,490$)$

CALL VLABL 'FLUENCE', 7 )

CALL TOUTST(2,LSMALL)

900 FORMAT(AI)

C PLOT LOG GAIN AS FUNCT OF DISTANCE

CALL TOUTST( 2 , LCLEAR)

WR I TE ( 5, 115) GL, GAMMA (1), GRATIO, GPER(NSTEPS+1), HSTEPS, DELTA,

1 GAMMA (NSTEPS+1), FNREL, GPAR (NSTEPS+1)

$2 \% 5$

FORMAT I $I X$ 'GAIN*LENGTH ',F6.2,5X, 'INPUT FLUEHCE = 'F8.4.

$15 X, ' G A I N$ RATIO=',F8, $4 / 11 X+L N(G P E R)=', F B .4,6 X, ' L N(G P A R\}=1, F 8,4)$

CALL BINIT

CALL CHECK(XPLOT, GPERPT)

CALL LINETI5232)

CALL DSPLY XPLOT, GPERPT)

CALL LINET $\{1\}$

CALL CPLOT (XPPLOT,GPARPT)

CALL TOUTSTS $2, L B I G)$

CA!L MOVABS $(490,60)$

CALL HLABL:'LENGTH',6)

CALL MOVABS $(5 \%, 540)$

CALL VLABL\{'GAIN COEFF',10\}

CALL TOUTST(2,LSMALL)

READ (5,9פD) IWAIT

GO TO 1

$c$
$C$
$c$
$c$

END

FUNCTION ERFMOD (GAMMA)

C THIS FUNCTION RETURNS A FACTOR THAT IS CFTEN FOUND IN THE COMMON DELTA

DIMENS: Ot! $A(5)$

DATA P.A(1).A(2),A(3),A(4),A(5)/.3275911,.2548296, 
$1-.2841967,1.4214137,-1.4531520,1.0614054 /$

DELTMP $=(1 .-.6666667 * D E L T A)$

$X=S Q R T$ ( GAMMA*DEL TA/DELTMP)

$T=1 . /(1 .+(p * x))$

ERF $\boldsymbol{D} \boldsymbol{g}$.

DO 1 ह日g $I=1,5$

$E R F=E R F+(A\{I) *(T * * 1))$

119 CONTINUE

THIS ERFMOD-1.-\{ERF*EXP(-\{X**2\})\}

C THIS IS THE ERROR FUNCTION AT THIS ROIHT

C MULTIPLY BY SORT(PI $1 / 2$

$E R F M O D=E R F M O D * .8862269$

ERFMOD- (ERFNOD / X) EXXP (-GAMMA* (1. -DELTA) /OELTMP)

RETURN

END 


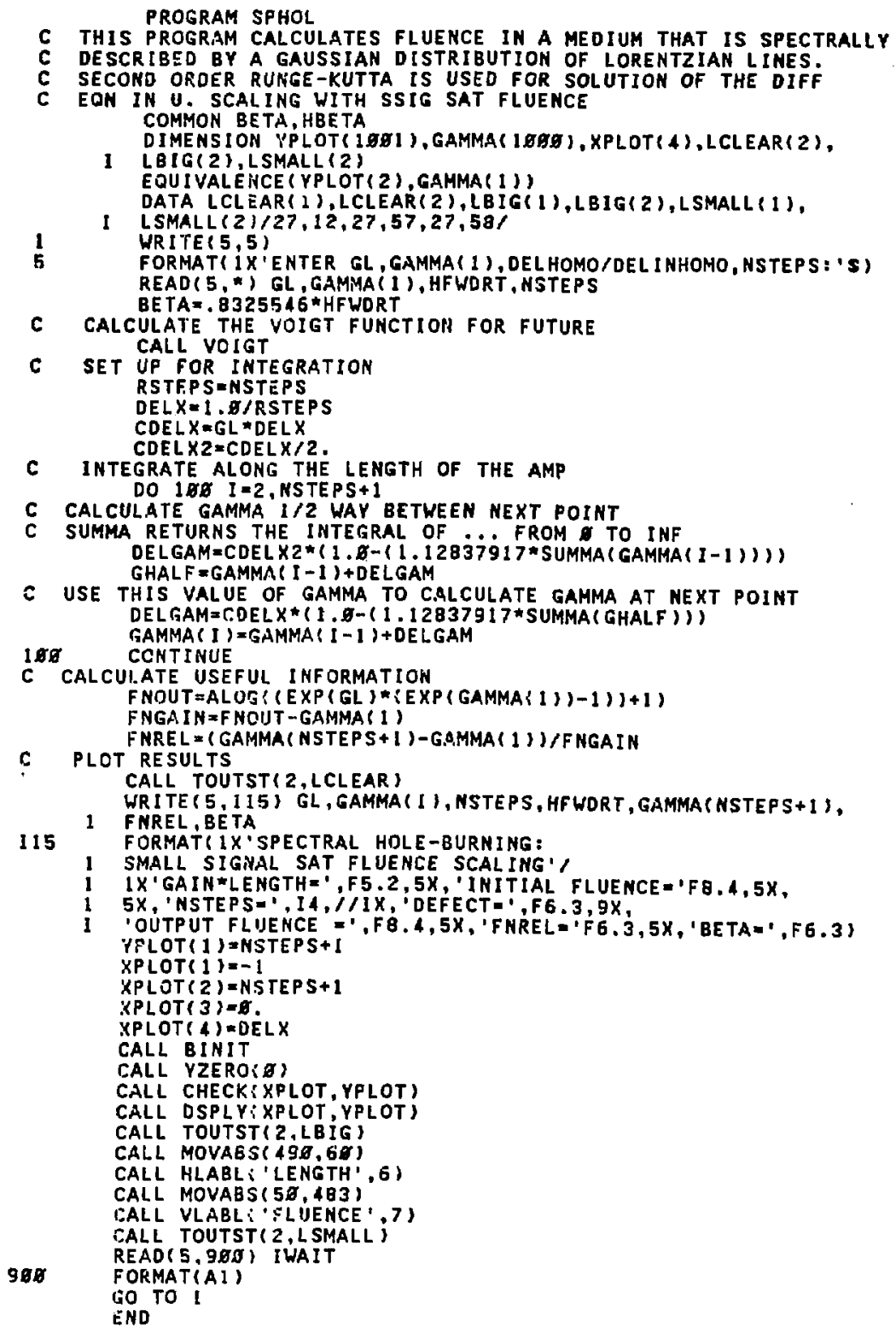


c

C TH UROUTINE VOIGT

C W: I ETAFE FOR VALUES OF BETA PASSED THROUGH COMMON COMMON BETA, HBETA

DIMENSION A(5)

DATA A(1),A(2),A(3),A(4),A(5)/.2548296,-.2844967,1.4214137,

$1-1.4531529,1.0614054 / 2 / .3275911 /$

$T=1.0 /(1 . \theta+(Z * B E T A))$

HBSTA=D.

DO IES III, 5

$100 \quad$ HBETA=HBETA+A I $) \star(T * \star I)$

C MAKE HBETA THE VALUE WE NEED

HBE TA $\approx$ HBETA*BETA* 1.772454

RETURN

c

END

FUNCT ION SUMMA (GAMMA)

C THIS FUNCTION USES TRAPEZOIDAL RULE AND INTEGRATES FROH COMMON BETA,HEETA

SUMMA $=$ D.

$V=\boldsymbol{D}$

DEL V = .E2

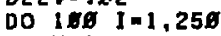

$V 2=V * * 2$

VDB $2-V 2 /(B E T A *+2)$

EXPON=-(V2+(GAMMA/(HBETA $(1+$ VDBZ $S))$

SUMMA $=$ SUMMA +EXP(EXPON)

$V=V+D E L V$

1 eg CONTINUE

C SUBTRACT I/2 OF FIRST POINT TO MAKE TRAPEZOIOAL RULE (I/2 OF LAST POINT-E) SUMMA = SUMMA- $5 \star E X P(-$ GAMMA $/ H B E T A)$

SUMMA = . .2* SUMMA

RETURN

END 
PROGRAM HOL

5 THIS PROGRAM CALCULATES FLUENCE IN A MEDIUM THAT IS ORIENTATIONALLY

C AND SPECTRALLY INHONOGENEOUS.

C SECOND ORDER RUNGE-KUTTA IS USED FOR SOLUTIOH OF THE DIFF

C EON IN U. THIS PRUGRAM IS FOR CONSTANT INITIAL SS GAIN

C FLUENCE IS SCALED IN TERMS OF SS SAT FLUENCE COMMON DELTA, EETA, B, HBETA, DELPAR, DELPER DIMENSION YPLOT 5 I I), GAMMÁ(5ED), XPLOT( 4$), \operatorname{LCLEAR}(2)$.

1 LBIG( 2$), L S M A L L(2)$, GPAR (5ED), GPARPT(5E1), GPER(5EE), GPERPT(5E1) EQU I VALENCE (YPLOT $(2)$, RAMMA( $i)$ ), (GPARPT $(2)$, GPAR $(1)$ ).

1 (GPERPT( 2 ), GPER(1)) DATA LCLEAR ( 1 ), LCLEAR( 2 ), LBIG( 1$), \operatorname{LBIG}(2), \operatorname{LSMALL}(1)$,

$1 \quad L S M A L L(2) / 27,12,27,57,27,58 /$

1 WRITE $(5,5)$

5 FORMAT ( IX'ENTER GL, GAMMA ( 1 ), FWHFMX, DELTA, NSTEPS: ' $\$$ )

READ ( 5,*) GL, GAMMA 1 ), FWHFMX, DELTA, NSTEPS

C CALCUI.ATE OFTEN USED QUANTITIES $B E T A=.83255: 6$ *WHFMX

C VOIGT FUNCTION IS ACCURATE ONLY FOR CERTAIN BETA

IF (BETA.GT.3.OR.BETA.LT.E.E5) GO TO 1

CALL VOIGT

C CALCULATE PARAMETER B NECESSARY FOR OBLSUM $B=(1 .-.666667 * D E L T A) * H B E T A$

c

SET UP FOR INTEGRATION

GPAR $(1)=\varnothing$

GPER $(1)=5$

RSTEPS $=$ NSTEPS

DEL $X=1, G / R S T E P S$

$C D E L X=G L * D E L X$

C CDE $L X 2=\operatorname{COE} L X / 2$.

C INTEGRATE ALONG THE LENGTH OF THE AMP DO 1 ED $I=2$, NSTEPS+1

C CALCULATE GAMMA $1 / 2$ WAY BETWEEN NEXT POINT DEL GAM $=C D E L X 2 *(1.0-D B L S U M(G A M M A(I-1))$ GHALF = GAMMA ( I - 1 ) + DELGAM

C CALCULATE GAIN COEFFICENTS AT HALF WAY POINT TOO.

C DELPAR AND DELPER ARE UPDATED EACH TIME DBLSUM IS USED GPARHF = GPAR $(1-1)+($ DELPAR $\$$ CDELXZ)

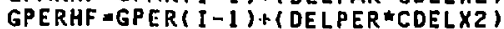

C USE THIS VALUE OF GAMMA TO CALCULATE GAMMA AT NEXT POINT DEL GAM $=$ CDE $L *(1, \varnothing-D B L S U M(G H A L F))$ GAMMA ( I ) $=$ GAMMA ( 1 - 1 ) + DE LGAM

C CALCULATE GAINS FROM HALF WAY POINT TO NEXT POINT GPAR (I) = GPARHF + (CDELX2*DELPAR) GPER (I ) = GPERHF + (CDELX2 WDELPER)

WR I TE ( 5,95 ) DELGAM,GAMMA( I), DELPAR, GPAR ( I),DELPER, GPER ( I)

95 TORMAT $(1 X, 6 F 18.5)$

1 EO CONTINUE

C CALCULATE USEFUL INFO SUCH AS MEASURED PROBE GAIA RATIO GRAT IO-EXP ( GPER(NSTEP S+1) I/EXP \{GPAR (NSTEPS+1) )

C CALCULATE RESULTS FOR FRANTZ NODVICK SYSTEM AND COMPARE FNOUY $=A L O G(\{$ EXP(GL $) *(E X P(G A M M A(1))-1))+1)$ FNGA I $N=$ F NOUT - GAMMA ( 1$)$ FNRE L = ( GAMMA ( NSTEPS + 1) -GAMMA( 1 ) )/FNGAIN

C PLOT RESULTS CALL TOUTST( 2, LCL.EAR)

WRITE( 5, i 15 ) GL, GAMMA( 1), GRATIO, GPER(NSTEPS+1), DELTA, NSTEPS,

1 GAMMA( MSTEPS+1), FNREL, GPAR(NSTEPS+1). FWHFMX

115 FORMATC 1 X HOL $\%$

1 IX'GAIN*LENGTH=', F5.2,5X, ' INITIAL FLUEACE = 'FB.4,

$15 X, ' G A I N$ RATIO=',F6,3,5X, 'LN(GPER)=',F8,4,5X, ' DELTA =',F6,3,

1 /IX,'NSTEP $=1,13,12 \mathrm{X}$. 


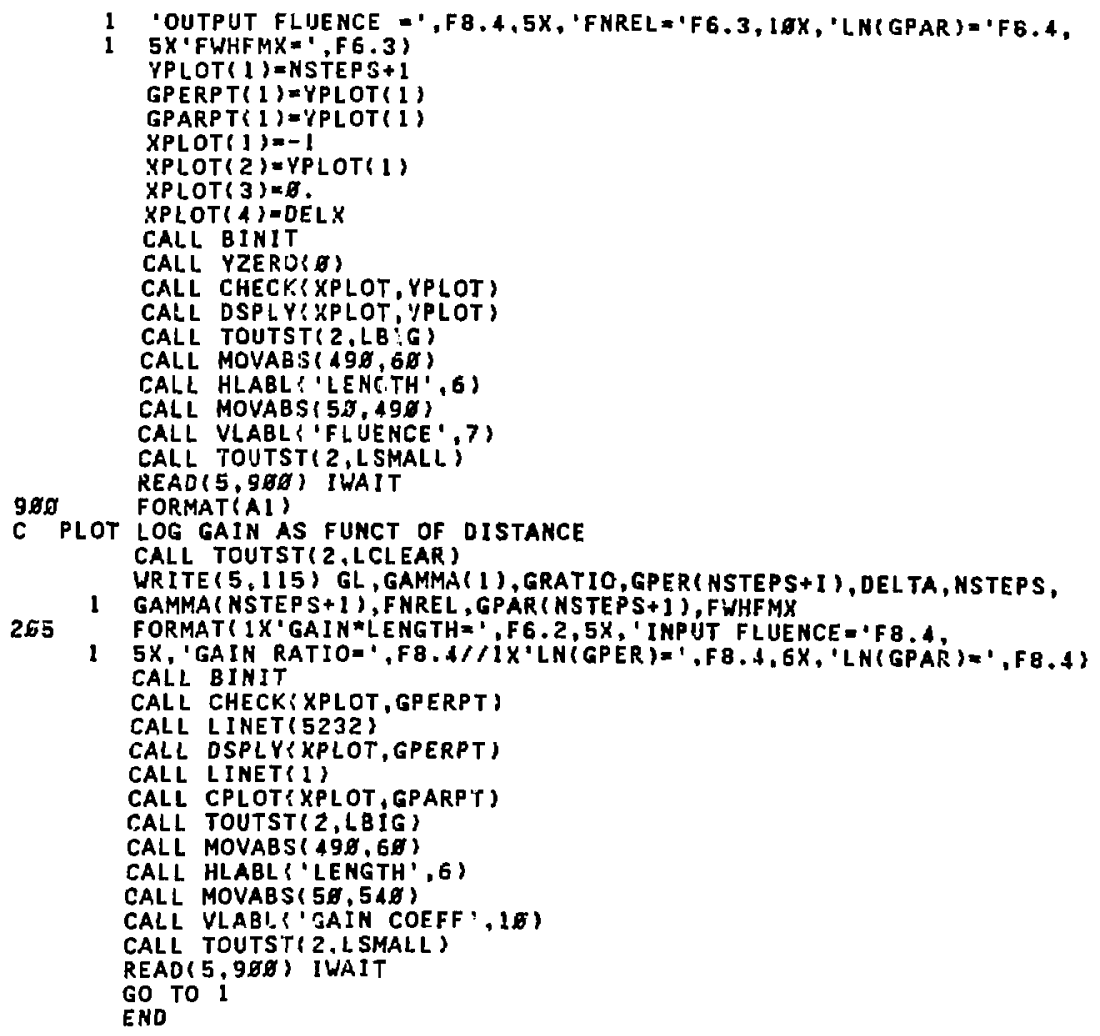




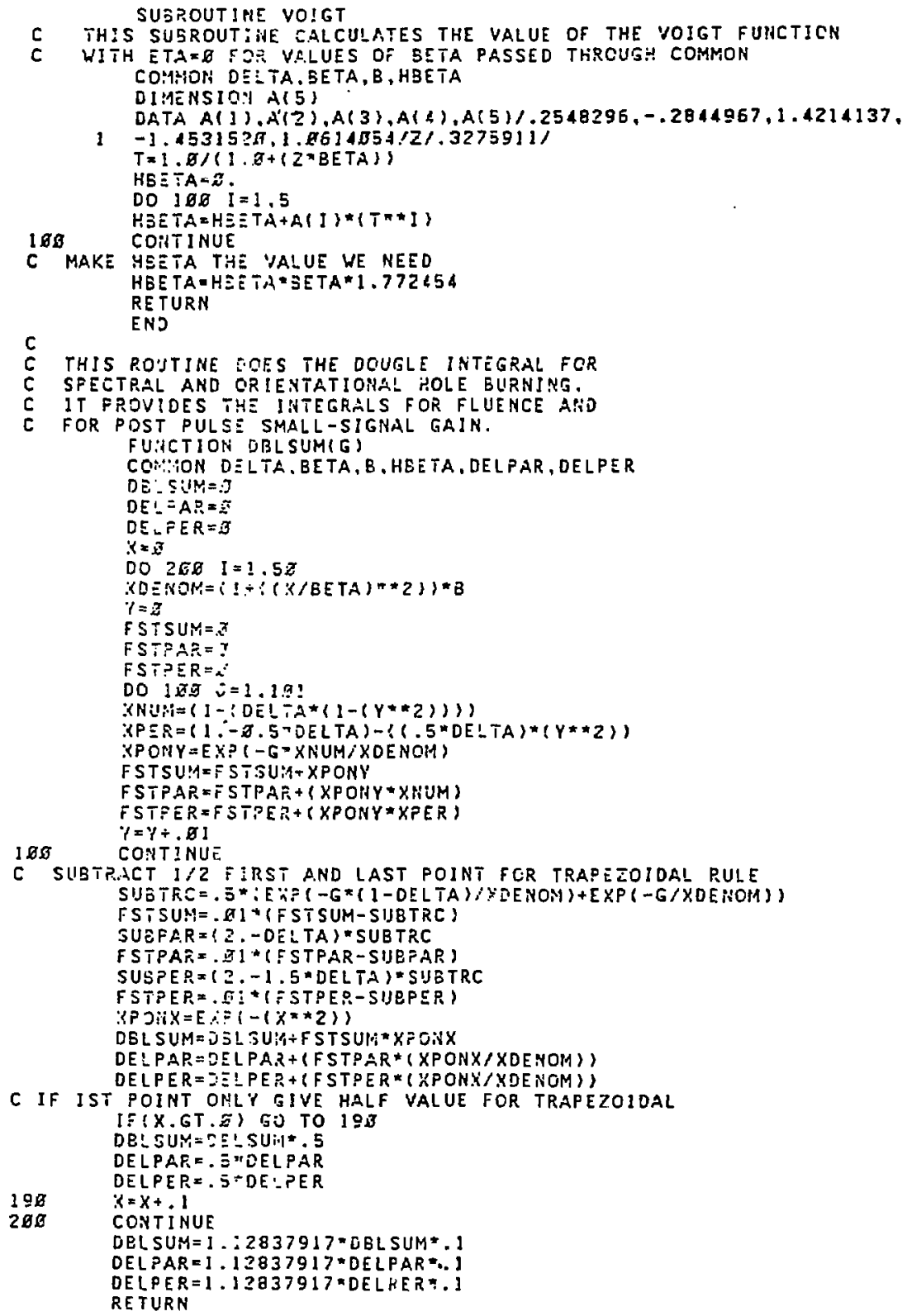

